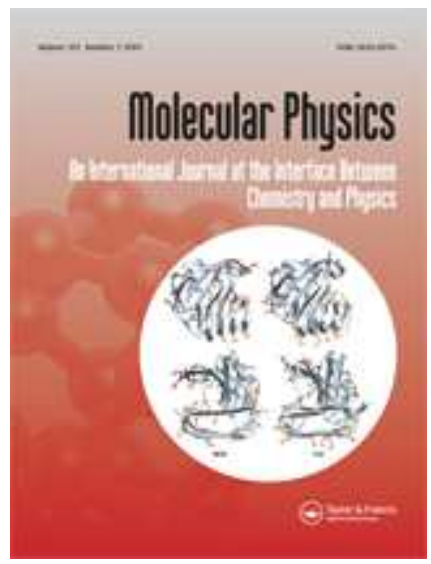

\title{
Precision measurements with polar molecules: the role of the black body radiation
}

\begin{tabular}{|c|c|}
\hline Journal: & Molecular Physics \\
\hline Manuscript ID: & TMPH-2007-0060.R1 \\
\hline Manuscript Type: & Full Paper \\
\hline $\begin{array}{r}\text { Date Submitted by the } \\
\text { Author: }\end{array}$ & 07-May-2007 \\
\hline Complete List of Authors: & $\begin{array}{l}\text { Vanhaecke, Nicolas; Laboratoire Aimé Cotton } \\
\text { Dulieu, Olivier; Laboratoire Aimé Cotton }\end{array}$ \\
\hline Keywords: & $\begin{array}{l}\text { black body radiation, precision measurements, absorption rate, } \\
\text { light shift, cold polar molecules }\end{array}$ \\
\hline \multicolumn{2}{|c|}{$\begin{array}{l}\text { Note: The following files were submitted by the author for peer review, but cannot be converted } \\
\text { to PDF. You must view these files (e.g. movies) online. }\end{array}$} \\
\hline Vanhaecke_Dulieu.tex & \\
\hline
\end{tabular}

\section{s ScholarONE \\ Manuscript Central}


Molecular Physics, Vol. 105, No. 1, 10 January 2007, 1-11

\title{
Precision measurements with polar molecules: the role of the black body radiation
}

\author{
N.VANHAECKE* and O. DULIEU \\ Laboratoire Aimé Cotton, CNRS, Bât. 505, Campus d’Orsay, 91405 Orsay Cedex, France
}

(v3.1 released January 2007)

\begin{abstract}
In the perspective of the outstanding developments of high-precision measurements of fundamental constants using polar molecules related to ultimate checks of fundamental theories, we investigate the possibly counterproductive role of the black body radiation on a series of diatomic molecules which would be trapped and observed for long durations. We show that the absorption of the black body radiation at room temperature may indeed limit the lifetime of trapped molecules prepared in a well-defined quantum state. Several examples are treated, corrresponding to pure rotational absorption, pure vibrational absorption or both. We also investigate the role of the black body radiation induced energy shift on molecular levels and how it could affect high-precision frequency measurements.
\end{abstract}

Keywords: black body radiation, precision measurements, absorption rate, light shift, cold polar molecules.

\section{Introduction}

Over the last decades more and more efforts have been put on searching experimentally for "new physics" breaking the fundamental $C, P, T$ symmetries. High-energy experiments have initiated this quest for physics beyond the Standard Model more than fourty years ago with the discovery of the $C P$-violation [1]. Since then not only high-energy experiments have been searching for parity violations, but also low-energy experiments based on high-precision measurements, as initially proposed in atomic systems [2]. For instance, recent results on the $P$ parity violation have been obtained probing the highly dipole-forbidden $6 S-7 S$ transition in cesium $[3,4]$.

This kind of precision measurement is especially eager to take advantage of the most sensitive tools to gain in precision and to reduce systematic errors. In that respect, cold atoms have brought huge improvements in several high-precision investigations, and nowadays cold molecules hold even greater promise. In such experiments, interferometers and quantum beats with atoms and molecules are often used and very long interrogation times (up to a few seconds) are needed to let the phase being accumulated in the interferometer. The search for time dependence of the fundamental constants needs particularly highprecision measurements since it is looking for small relative drifts. In the search for a time dependence of the fine structure constant, which would imply the violation of both Lorentz invariance and CPT symmetry, the best measurement to date has been achieved through comparisons of atomic frequency standards [5]. The recent high-resolution spectroscopy of cold $\mathrm{OH}$ radicals in a Stark-decelerated beam combined with astrophysical measurements of $\mathrm{OH}$ megamasers gives hope to yield an even better determination of the time variation of the fine structure constant [6]. The time dependence of the proton-electron mass ratio is planned to be investigated by measuring extremely precisely the inversion splitting in the ammonia molecule in a molecular fountain [7]. A tremendously exciting search for a possible permanent electric dipole moment (EDM) of atoms, nucleons and elementary particles like electron and muon is being carried out [8-12], as its existence would reveal a violation of the time-reversal invariance. To date, the lowest limit on the value of a possible electron EDM has been established using the thallium atom [11]. Molecules are expected to open the way to even more precise measurements and many efforts are put nowadays to set

$\overline{{ }^{*} \text { Corresponding author. Email: nicolas.vanhaecke@lac.u-psud.fr }}$ 
Figure 1. Spectral energy density of the BBR at room temperature $(300 \mathrm{~K})$. On the right side are schematically depicted the typical energy ranges for vibrational and rotational transitions in heavy and light molecules.

up experiments with cold beams of heavy polar molecules such as $\mathrm{YbF}, \mathrm{PbO}$ or $\mathrm{PbF}$ [13,14]. Cold slowed or trapped molecules offer even better prospects, allowing very long interrogation times on the order of several seconds.

The accuracy of such high-precision measurements has become so amazingly high, that every possible little disturbance has to be considered very carefully. So has to be the black body radiation (BBR), always present in an experimental environment. Atoms in the ground state are normally immune to the absorption of black body photons at room temperature, since such photons do not carry enough energy to induce a dipole-allowed transition. This is not the case in Rydberg species, as it is known for a long time [15]. A distribution of molecular ions has also shown to evolve toward a thermal equilibrium at the environment temperature through exchange of black body photons [16]. It has been proposed to use BBR to cool down internal states of molecular ions [17] and very recently laser-trapping of radium took advantage of the BBR as repumping light [9]. Recently BBR absorption rates have been experimentally measured in trapped clouds of cold $\mathrm{OH}$ and OD radicals [18]. The presence of the BBR induces also a shift of the atomic resonances, which is a well-known effect on frequency measurements in atomic clocks. Such a light shift is now one of the biggest uncertainties in high-precision measurements of frequencies [19-21]. So far it has not been observed in molecular systems.

In this paper we investigate the possible limitations induced by the BBR on experiments aiming at interrogating molecular systems over long time intervals. Such experiments often require heavy molecules with large permanent dipole moments which make them very sensitive to the presence of any external electric field, like the BBR electric field. We first recall in section 2 the different transitions which can be induced by BBR in molecules, emphasizing through a simple model the crucial role of both the magnitude and the variation of the permanent electric dipole moment of the molecule with the internuclear distance. Then we explore several classes of molecules of relevance for running, planned or possible high-precision measurements: heavy fluoride radicals (section 3), strongly polar alkali dimers, alkali hydrides as well as sulfur oxide (section 4). We investigate in section 5 the role of the BBR-induced energy shift in two molecular systems of relevance now in the context of high-precision measurements: the YbF radical and the ammonia molecule.

\section{Absorption of black body radiation by diatomic molecules}

The spectral energy density of the BBR at a frequency $\nu$ and a temperature $T$ is given the Planck radiation law: 
where the summation is performed over all final states $f$ accessible from the initial state $i$ via an electric dipole transition at frequency $\nu_{f i}$. The details of the rovibronic structure of the quantum system are involved through the matrix elements $\mu_{f i}=\langle f|\mu| i\rangle$ of the dipole moment operator $\mu$ between the initial state $i$ and the final state $f$. We restrict ourselves to the study of heteronuclear (i.e. polar) diatomic molecules, assuming that both $i$ and $f$ states can properly be described within the Born-Oppenheimer (BO) approximation with total wave functions $\Psi_{i}^{B O}$ and $\Psi_{f}^{B O}$. The $\mathrm{BO}$ wave functions are written as products of electronic $(\psi)$, vibrational $(\chi)$, and rotational wave functions $(\Theta)$ :

$$
\Psi_{i, f}^{B O}=\psi_{\alpha_{i, f}}(R ;\{\vec{r}\}) \chi_{v_{i, f}}(R) \Theta_{J_{i, f}}(\Omega) .
$$

In eq.(3), the $\alpha_{i, f}, v_{i, f}$ and $J_{i, f}$ labels refer respectively to the electronic, vibrational, and rotational quantum numbers of the initial and final states. The electronic part depends on all electronic coordinates $\{\vec{r}\}$, and the rotational part on the angular coordinates $\Omega$ of the molecular axis in the space-fixed frame. We assumed that there is no coupling between vibrational and rotational degrees of freedom. The transition dipole moment $\mu_{f i}$ can be reformulated as the integral over the interatomic separation $\vec{R}$ :

$$
\mu_{f i}=\int \chi_{v_{f}}(R) \Theta_{J_{f}}(\Omega) \vec{\mu}_{f i}^{B O}(\vec{R}) \cdot \vec{\epsilon} \chi_{v_{i}}(R) \Theta_{J_{i}}(\Omega) d R d \Omega
$$

which accounts for the orientation of the instantaneous dipole moment for all electrons relative to the polarization $\vec{\epsilon}$ of the black body photon. The $R$-dependent transition dipole moment function $\vec{\mu}_{f i}^{B O}(\vec{R})$ is obtained after integration of the product $\psi_{\alpha_{f}} \mu \psi_{\alpha_{i}}$ on the electronic coordinates.

The energy distribution of the BBR spectrum peaks around $600 \mathrm{~cm}^{-1}$ at room temperature, which is not sufficient to induce any electronic transition starting from the ground state or from a low metastable state in diatomic molecules. Therefore we can safely ignore them, and restrict our attention to transitions between rotational or vibrational levels of a single electronic state $\alpha$. The transition dipole moment function now reduces to the permanent dipole moment function $\mu_{\alpha}(R)$ of the state $\alpha$ so that eq.(4) becomes

$$
\mu_{v^{\prime} J^{\prime} v J} \propto \int_{0}^{\infty} \chi_{v^{\prime}}(R) \mu_{\alpha}(R) \chi_{v}(R) d R \equiv \mu_{v^{\prime} v}
$$

where $v$ and $v^{\prime}$ (resp. $J$ and $J^{\prime}$ ) are the initial and final vibrational (resp. rotational) levels of the transition within the state $\alpha$. The proportionality factor in eq.(5) is the Hönl-London factor between the initial and the final state, which accounts for the initial and final rotational levels $J$ and $J^{\prime}$ and for the relative orientation of the molecular axis and of the polarization of the BBR electric field. We will see from eq. (5) that depending on the type of transition, the magnitude of $\mu_{v^{\prime} J^{\prime} v J}$ is determined either by the magnitude or by the $R$-dependence of the dipole moment function. The knowledge of molecular dipole 
moment functions generally require quantum chemistry calculations which are available over a wide $R$ range only for a few molecules of interest here. The value of this function at the equilibrium distance $R_{e}$ of the molecule and its first derivative around $R_{e}$ are also sometimes given in the literature. This information is actually sufficient to evaluate the BBR absorption rate, as in all cases considered here the vibrational ground state wave function -which peaks at $R_{e^{-}}$is of relevance. Indeed, the function $\mu_{\alpha}(R)$ can be expanded to the first order around $R_{e}$ and reads

$$
\mu_{\alpha}(R)=\mu_{\alpha}\left(R_{e}\right)+\left[\frac{d \mu_{\alpha}}{d R}\right]_{R=R_{e}}\left(R-R_{e}\right),
$$

where $\left[\frac{d \mu_{\alpha}}{d R}\right]_{R=R_{e}}$ is the first derivative of the electric dipole moment function evaluated at $R_{e}$. The scheme displayed in Fig.1 illustrates the various situations treated here. For light molecules, with a mass smaller than ten atomic mass units say, the typical energy for vibrational transitions often exceeds the peak of the BBR energy distribution, so that pure rotational transitions are dominant, the molecule remaining in its lowest vibrational level $(v=0)$. The constant term $\mu_{\alpha}\left(R_{e}\right)$ in eq.(6), i.e. the magnitude of the dipole moment, brings the main contribution to $\mu_{v J^{\prime} v J}$ and the integral in eq.(5) almost reduces to $\mu_{\alpha}\left(R_{e}\right)$. In contrast, for heavier molecules the energy of rotational transitions corresponds to the low energy part of the BBR distribution, so that vibrational transitions are favoured. Therefore $\mu_{v^{\prime} J^{\prime} v J}$ (with $v^{\prime} \neq v$ ) mainly depends on the slope of the dipole moment function, and no more on its magnitude, due to the mutual orthogonality of vibrational wave functions. A further simplification can be made if the vibrational (harmonic) constant $\omega_{e}$ of the relevant potential curve is known. The radial integral between two adjacent vibrational levels in eq.(5) can be analytically evaluated for a harmonic potential:

$$
\mu_{v+1, v}=\sqrt{\frac{v+1}{2}} \sqrt{\frac{\hbar}{m \omega_{e}}}\left[\frac{d \mu}{d R}\right]_{R=R_{e}},
$$

where $m$ is the reduced mass of the molecule. These approximations break down if the potential well is strongly anharmonic, if the dipole moment function is notably nonlinear or too flat around $R_{e}$, or if couplings such as rovibrational couplings are not negligeable.

\section{BBR absorption rate for fluoride radicals: $\mathrm{BaF}, \mathrm{YbF}, \mathrm{HgF}$ and $\mathrm{PbF}$}

In heavy paramagnetic molecules such as $\mathrm{BaF}, \mathrm{YbF}, \mathrm{HgF}$ and $\mathrm{PbF}$ - of relevance for the search of the electron EDM - the vibrational wave function of low-lying vibrational levels extends typically over a small fraction of an atomic unit ( $\left.1 \mathrm{a} . \mathrm{u} . \equiv a_{0}=0.0529177 \mathrm{~nm}\right)$, so that the dipole moment function of the electronic ground state can safely be considered linear over this extension. Therefore, the strength of the electric dipole transition between two consecutive vibrational states can be estimated following eq.(7) as soon as the derivative of the electric dipole moment function at the equilibrium distance is known.

Diatomic lanthanide compounds possess partially filled $4 f$ and $5 d$ shells and involve strong inter-shell couplings, which generate very complicated spectra. Their study is therefore challenging for both theoretical and experimental investigations. Among the molecules mentioned above, $\mathrm{YbF}[22,23]$ and $\mathrm{PbF}[24]$ have been studied from $a b$ initio theory and dipole moment function of $\mathrm{YbF}$ and its value and first derivative for $\mathrm{PbF}$ at the equilibrium internuclear distance have been derived.

Extensive quantum chemistry studies on alkaline earth monohalides are available mainly for the lighter ones (involving $\mathrm{Be}, \mathrm{Mg}$, $\mathrm{Ca}$, Sr linked with $\mathrm{F}$ or $\mathrm{Cl}$ ), providing all necessary quantities for the present purpose, including the derivative of the permanent dipole moment [25]. Quantum chemistry calculations have also been devoted to $\mathrm{BaF}[26]$ and $\mathrm{HgF}$ [27] molecules, providing the main properties of the electronic ground state except the $R$-variation of its permanent dipole moment function. Fortunately, alkaline earth monohalides (hereafter labelled as Me-Hal) have been extensively studied in the past, since their theoretical 
Table 1. Equilibrium distances, vibrational and rotational frequencies, values of the dipole moment function and of its first derivative at the equilibrium distance for the dimers discussed throughout the paper. The state considered here is always the rovibrational ground state, unless otherwwise stated.

\begin{tabular}{l|l|l|l|l|l|l|l|l}
\hline Species & Initial state & $\begin{array}{l}R_{e} \\
\left(a_{0}\right)\end{array}$ & $\begin{array}{l}\omega_{e} \\
\left(\mathrm{~cm}^{-1}\right)\end{array}$ & $\begin{array}{l}B_{e} \\
\left(\mathrm{~cm}^{-1}\right)\end{array}$ & $\begin{array}{l}\mu_{e} \\
(\mathrm{D})\end{array}$ & $\begin{array}{l}\mu_{e}^{\prime} \\
\left(\mathrm{D} a_{0}^{-1}\right)\end{array}$ & Ref \\
\hline $\mathrm{YbF}$ & $X^{2} \Sigma^{+}$ & 3.80 & 502 & 0.24 & 3.55 & 3.09 & {$[22]$} \\
$\mathrm{BaF}$ & $X^{2} \Sigma^{+}$ & 4.09 & 469 & 0.21 & 3.51 & 4.52 & {$[36]$} \\
$\mathrm{HgF}$ & $X^{2} \Sigma^{+}$ & 3.87 & 489 & & 7.24 & 4.14 & \\
$\mathrm{PbF}$ & $X^{2} \Sigma^{+}$ & 3.94 & 530 & & 4.32 & 3.38 & {$[24]$} \\
\hline $\mathrm{LiRb}$ & $X^{1} \Sigma^{+}$ & 6.50 & 185 & & 4.15 & 0.14 & {$[37]$} \\
$\mathrm{LiCs}$ & $X^{1} \Sigma^{+}$ & 6.82 & 164 & & 5.44 & 0.45 & {$[37]$} \\
$\mathrm{NaRb}$ & $X^{1} \Sigma^{+}$ & 6.84 & 107 & & 3.30 & 0.20 & {$[37]$} \\
$\mathrm{NaCs}$ & $X^{1} \Sigma^{+}$ & 7.20 & 98.0 & & 4.61 & 0.34 & {$[37]$} \\
$\mathrm{KRb}$ & $X^{1} \Sigma^{+}$ & 7.64 & 75.5 & & 0.61 & 0.03 & {$[37]$} \\
$\mathrm{KCs}$ & $X^{1} \Sigma^{+}$ & 8.01 & 66.2 & & 1.90 & 0.11 & {$[37]$} \\
$\mathrm{RbCs}$ & $X^{1} \Sigma^{+}$ & 8.28 & 49.4 & & 1.23 & 0.07 & {$[37]$} \\
\hline $\mathrm{LiH}$ & $X^{1} \Sigma^{+}, J=1$ & & & & & & \\
$\mathrm{CsH}$ & $X^{1} \Sigma^{+}, J=1$ & 4.50 & 891 & 2.7 & 8.30 & 2.10 & {$[38]$} \\
$\mathrm{SO}$ & $X^{3} \Sigma^{-}, N=0, J=1$ & 2.80 & 1150 & 0.72 & 1.51 & 0.94 & {$[39]$} \\
\hline
\end{tabular}

description is underlined by a simple picture: such molecules are ionic-bond molecules, in which the lone electron evolves in the field of two atomic ions, namely the closed-shell doubly-charged metal cation $\mathrm{Me}^{++}$ and the halogen anion $\mathrm{Hal}^{-}$. The ligand approach $[28,29]$ assumes that the electronic structure of the system can be described with orbitals centered on the free $\mathrm{Me}^{++}$ion while the electrostatic field of the ligand $\mathrm{Hal}^{-}$ion is treated as a perturbation. The electrostatic model proposed by Rittner [30] accounts for the mutual polarization of the $\mathrm{Me}^{+}$and $\mathrm{Hal}^{-}$ions through induced dipole moments which reduce the primary permanent dipole moment of the molecule. However, due to the large polarizability of the $\mathrm{Me}^{+}$ion, higher order effects break this approximation down. The more elaborated electrostatic model of Törring et al [31] accounts for the strong hybridization of the external electron of the $\mathrm{Me}^{+}$ion induced by $\mathrm{Hal}^{-}$, which acts as a radial shift of the center of charge of the electron wave function. The induced dipole moments are modified accordingly in the expression of the net dipole moment of the molecule, whose derivative can also be derived. Finally, as pointed out in refs. $[26,31]$ an effective polarizability of the $\mathrm{Hal}^{-}$ ion should be introduced in such ionic molecules which accounts for the influence of the $\mathrm{Me}^{+}$ion on the somewhat floppy negative ion. The effective value of 4.7 a.u. is recommended in refs. $[26,31]$ for $\mathrm{F}^{-}$within the $\mathrm{CaF}$ and $\mathrm{BaF}$ molecules, indeed much smaller than the free ion polarizability (about 16 a.u. [32,33]).

Using the value 81 a.u. of ref. [31] for the $\mathrm{Ba}^{+}$polarizability, the ionic model yields for $\mathrm{BaF}$ at the equilibrium distance $\left(R_{e}=4.09\right.$ a.u.) of its $X^{2} \Sigma^{+}$electronic ground state $\mu\left(R_{e}\right)=1.38$ a.u. (close to the experimental value 1.24 a.u. [34]) and $\left[\frac{d \mu}{d R}\right]_{R=R_{e}}=1.78$ a.u.. For $\mathrm{HgF}$ in its $X^{2} \Sigma^{+}$electronic ground state $\left(R_{e}=3.87\right.$ a.u.) the same model leads to $\mu\left(R_{e}\right)=2.85$ a.u. and $\left[\frac{d \mu}{d R}\right]_{R=R_{e}}=1.63$ a.u., taking the $\mathrm{Hg}^{+}$ polarizability 19 a.u. from ref. [35].

The structure parameters used for the BBR absorption rate calculations are collected in Table 1. We found rates slightly smaller than $1 \mathrm{~s}^{-1}$ (see Table 2), suggesting that the lifetime of a molecular sample in a well-defined initial state could be limited by BBR. In the near future, molecular interferometry for precision measurements will be performed in traps, which offer the opportunity to keep the molecules for sconds and hence in an interferometry experiment to let the phase being built for much longer times than in a beam experiment. Traps are therefore a very promising tool for measuring very small level energy differences with a molecular interferometer, as planned for instance with $\mathrm{YbF}$ and $\mathrm{PbF}$. If seconds are needed to let the phase build up, cycles of absorption-emission are then induced by the BBR and limit the constrast of the interferometer by breaking its coherence.

As a check of such predictions, we also calculated the BBR absorption rate for $\mathrm{YbF}$ and $\mathrm{PbF}$ using the ionic model and found a good agreement with the calculation using the derivatives of the dipole moment 
function from refs. $[22,23]$. Moreover, the influence of the inaccuracy on the static polarizability of the ions, which determines the molecular dipole moment, is exemplified with $\mathrm{HgF}$ and $\mathrm{BaF}$. Indeed, the $\mathrm{F}^{-}$effective polarizability depends a priori on the accompanying positive ion. In particular, the dipole moment of $\mathrm{HgF}$ (2.85 a.u.) presently obtained using the $\mathrm{Hg}^{+}$polarizability from ref. [35] (19 a.u.) is about $70 \%$ larger than the one given in ref. [27]. If we use the ionic model to adjust the dipole moment of $\mathrm{HgF}$ to the experimental value, the $\mathrm{F}^{-}$effective polarizability becomes close (14a.u.) to the one of the free ion. The derivative of the $\mathrm{HgF}$ dipole moment around $R_{e}$ increases with the $\mathrm{F}^{-}$effective polarizabilty, increasing the $\mathrm{BBR}$ absorption rate only from $0.47 \mathrm{~s}^{-1}$ to $0.86 \mathrm{~s}^{-1}$. Note also that Angstmann et al [35] computed a value of 122 a.u. for the $\mathrm{Ba}^{+}$polarizability, significantly higher than in ref. [31]. Introducing this value in the ionic model then yields a $\mathrm{F}^{-}$effective polarizability of 1.3 a.u. to recover the experimental dipole moment from [34]. We clearly reach here the limits of the validity of the ionic model but this does not significantly change the conclusions of our analysis.

\section{BBR absorption rates for alkali dimers, alkali hydrides and sulfur oxide}

Polar molecules are expected to exhibit long-range dipole-dipole interactions. One expects to be able to characterize the dipole-dipole interactions by studying collisions in a cold sample of polar molecules, just like were investigated the details of interactions between cold atoms in the late 90' [40]. Cold (half)collisions could also be studied by controlling the orientation of the molecules with external electric fields. Polar molecules are also promising candidates for quantum computing [41,42].

Great experimental efforts are put on the production of ultracold heteronuclear alkali dimers, which possess rather large dipole moments [37]. They are formed experimentally from trapped ultracold atoms and are therefore obtained at very low temperatures, much lower than in experiments starting from a molecular beam. The disadvantage of this technique is that it has so far adressed only alkali dimers. Up to now densities of ultracold polar alkali dimers are still low, but several group head toward accumulating and trapping these dimers in order to start investigating molecule-molecule collisions [43-45].

Many efforts are done nowadays on cold intense beams of alkali hydrides [46]. The lightest of them, LiH, could be stopped with a Stark decelerator in order to perform further sympathetic or evaporative cooling in a trap. However, in the samples of trapped molecules obtained up to now [47], densities are still too low, and temperature still too high to observe cold collisions.

Due to their large dipole moment, these molecules can undergo vibrational and rotational BBR induced electric dipole transitions within their electronic ground state. Nevertheless, for all alkali dimers, rotational constants are on the order of a fraction of a wavenumber, where the spectral energy density of the BBR is very small (see Figure 1). Only vibrational transitions are therefore of relevance. The vibrational matrix elements of the dipole moment were evaluated using the Fourier grid Hamiltonian method [48] taking as input data electronic ground state potentials and dipole moment functions provided by ref. [37]. We performed the calculations for several alkali dimers (see Table 2). For most of them, the absorption of black body photons cannot be noticeable at room temperature even for extremely long trapping times of several minutes which are reachable if special care is taken to reduce the background gas pressure. A noticeable exception to this is the case of LiCs. The black body limited lifetime of LiCs in its ground state at room temperature is only on the order of one minute, which constitutes a limitation in the investigation of collisions in a trapped cloud of LiCs and further evaporative cooling [44].

The lowest level in which $\mathrm{LiH}$ is foreseen to be efficiently manipulated in an electrostatic deceleration and trapping process is the $v=0, J=1$ rovibrational level of its $X^{1} \Sigma^{+}$electronic ground state. The pumping rate at room temperature due to vibrational transitions is rather small $\left(0.06 \mathrm{~s}^{-1}\right)$ since the vibrational constant is quite high $\left(\approx 1400 \mathrm{~cm}^{-1}\right)$. However, the black body photons are quite efficient at pumping the $v=0, J=1$ level to the $v=0, J=2$ level at a rate of $1.26 \mathrm{~s}^{-1}$ and at depumping it down to the $v=0, J=0$ level at a rate of $0.163 \mathrm{~s}^{-1}$. Moreover, the spontaneous emission depletes the $v=0, J=1$ level which decays to the $v=0, J=0$ level with a rate of $0.036 \mathrm{~s}^{-1}$. Finally this leads to an overall lifetime of LiH in the $v=0, J=1$ level of about $650 \mathrm{~ms}$ at room temperature. This harms long trapping of $\mathrm{LiH}$ in this state and therefore all cooling processes that could follow like evaporative, sympathetic or cavity-assisted cooling. Even at $77 \mathrm{~K}$ the lifetime increases only to about $3 \mathrm{~s}$, still limited by the black body assisted rotational transitions. 
Table 2. BBR absorption rate at $300 \mathrm{~K}$ for selected heavy diamagnetic polar molecules used in various precision measurement experiments, for selected alkali dimers and for $\mathrm{LiH}, \mathrm{CsH}$ and $\mathrm{SO}$. For all calculated rates and lifetimes the initial state is the vibrational ground state $(v=0)$. Values for KRb (resp. $\mathrm{RbCs})$ are in agreement with previous calculations of ref. [50] (resp. [51]).

\begin{tabular}{l|l|l|l|l}
\hline Species & Initial state & $\Gamma_{\text {rot }}\left(\mathrm{s}^{-1}\right)$ & $\Gamma_{\mathrm{v} i b}\left(\mathrm{~s}^{-1}\right)$ & Lifetime (s) \\
\hline $\mathrm{YbF}$ & $X^{2} \Sigma^{+}$ & - & 0.26 & 3.8 \\
$\mathrm{BaF}$ & $X^{2} \Sigma^{+}$ & - & 0.60 & 1.7 \\
$\mathrm{HgF}$ & $X^{2} \Sigma^{+}$ & - & 0.47 & 2.1 \\
$\mathrm{PbF}$ & $X^{2} \Sigma^{+}$ & - & 0.29 & 3.4 \\
\hline $\mathrm{LiRb}$ & $X^{1} \Sigma^{+}, J=0$ & - & $8.010^{-3}$ & 125 \\
$\mathrm{LiCs}$ & $X^{1} \Sigma^{+}, J=0$ & - & $1.710^{-2}$ & 59 \\
$\mathrm{NaRb}$ & $X^{1} \Sigma^{+}, J=0$ & - & $7.310^{-4}$ & $1.410^{3}$ \\
$\mathrm{NaCs}$ & $X^{1} \Sigma^{+}, J=0$ & - & $1.710^{-3}$ & 600 \\
$\mathrm{KRb}$ & $X^{1} \Sigma^{+}, J=0$ & - & $7.510^{-6}$ & $1.310^{5}$ \\
$\mathrm{KCs}$ & $X^{1} \Sigma^{+}, J=0$ & - & $8.210^{-5}$ & $1.210^{4}$ \\
$\mathrm{RbCs}$ & $X^{1} \Sigma^{+}, J=0$ & - & $1.510^{-5}$ & $6.710^{4}$ \\
\hline $\mathrm{LiH}$ & $X^{1} \Sigma^{+}, J=1$ & 1.52 & $6.010^{-2}$ & 0.63 \\
$\mathrm{CsH}$ & $X^{1} \Sigma^{+}, J=1$ & 1.55 & 1.2 & 0.36 \\
$\mathrm{SO}$ & $X^{3} \Sigma^{-}, N=0, J=1$ & - & $8.310^{-3}$ & 120 \\
\hline
\end{tabular}

In order to investigate dipole-dipole interactions, the best alkali hydride is $\mathrm{CsH}$ (with a dipole moment of $8.3 \mathrm{D}$ ), for which one can expect strong interactions between $\mathrm{CsH}$ molecules through the dipole-dipole interaction. However its dipole moment to mass ratio is not encourageous in the perspective of a Stark deceleration. Nevertheless, under the same conditions as for $\mathrm{LiH}$ above, we found that the contribution of the vibrational transition to $v=1$ levels amounts to $1.17 \mathrm{~s}^{-1}$, since the vibrational frequency $\left(891 \mathrm{~cm}^{-1}\right)$ is quite close to the peak of the black body spectrum. The rotational transitions contribute to the depletion of the $v=0, J=1$ level with the following rates: $0.56 \mathrm{~s}^{-1}$ for $J=2 \leftarrow J=1$ and $0.071 \mathrm{~s}^{-1}$ for $J=0 \leftarrow J=1$, while the spontaneous emission rate is negligibly small $\left(0.0056 \mathrm{~s}^{-1}\right)$. In total, at room temperature the $v=0, J=1$ level is depleted at a rate of $1.80 \mathrm{~s}^{-1}$. At $77 \mathrm{~K}$ this rate drops down to $0.16 \mathrm{~s}^{-1}$, essentially given by the $J=2 \leftarrow J=1$ black body assisted transition.

Cold chemistry involving free radicals is a blooming field and is expected to bring new insight on reactive collision and dissociation. As an experiment aims at producing cold SO radicals and O atoms produced from near-threshold dissociation of trapped $\mathrm{SO}_{2}$ [49], it is worth calculating also the BBR absorption rate of the SO radical in its ground state. The lowest electronic states have been studied and potential energy curves and dipole moments have been computed [39]. We estimate that the BBR absorption rate, solely due the first vibrational transition $\left(1120 \mathrm{~cm}^{-1}\right)$, is $8.310^{-3} \mathrm{~s}^{-1}$, in agreement with [18]. This should not affect the trapping time of $\mathrm{SO}$ radicals in an electrostatic or in a magnetic trap.

\section{The black body radiation induced shift in molecules}

According to quantum electrodynamics theory, light can not only be absorbed by a massive particle, but it also shifts the level energies of the particle [52]. The BBR is known for a long time to be responsible for a shift of the atomic hyperfine splittings [53], which is of extreme relevance for atomic clocks [19-21]. For atoms in their ground state, all electric dipole allowed transitions require photons with much more energy than available in the BBR spectrum at room temperature. Therefore the BBR electric field acts on the atom through its static polarizability, exactly like a dc electric field does, and only the amplitude of the black body electric field is then of importance. As shown in the previous sections, a molecule likely absorbs resonantly a black body photon through an electric dipole transition. Therefore the BBR does not act on the molecule simply like a dc electric field but the entire BBR spectrum has to be considered.

The black body is a very incoherent source of photons, with a coherence time of about $h / 4 k_{B} T \approx 40 \mathrm{fs}$ at room temperature [54], which is much smaller than the typical absorption time of a black body photon by the molecule (see rates of Table 2 and ref. [18]). Therefore, according to ref. [52], the BBR-induced 
frequency shift of the level $i$ reads:

$$
\Delta_{i}=\frac{4 \pi}{3 \epsilon_{0} h c^{3}} \sum_{f \text { states }} \mathcal{P} \int d \nu \frac{\nu^{3}}{e^{h \nu / k_{B} T}-1} \frac{\mu_{f i}^{2}}{\nu-\nu_{f i}}
$$

with the same notations as in section 2 , and where the symbol $\mathcal{P} \int$ holds for the Cauchy principal part integral.

Molecular interferometers constitute excellent tools to quantify very small energy differences or measure very accurately transition frequencies. First, the experiments carried out on $\mathrm{YbF}$ in order to detect the electron EDM ultimately need to measure frequencies down to a precision of tens of $\mu \mathrm{Hz}[13]$. In that reference, the effect of the BBR is not explicitely discussed. We found that the BBR-induced shift due to rotational electric dipole allowed transitions $(N=1 \leftarrow N=0)$ is on the order of tens of $\mathrm{mHz}$, while the contribution to the shift of vibrational transitions is on the order of a few $\mathrm{mHz}$, much larger than the above needed precision. However, a careful analysis of the paper by Hudson et al [13] shows that the ratio of the differences of phases measured under various external field conditions is insensitive to the first order to the induced light shifts. Indeed, each individual phase measured in the YbF interferometer depends on the difference between light shifts induced on two Zeeman sublevels, which is proportional (with an extremely small factor of about $10^{-13}$ ) to their energy difference. This ensures that a BBR-induced correction to the electron EDM could be at maximum $10^{13}$ times smaller than its actual value, hence totally undetectable.

The discussion above suggests that a BBR induced light shift could play a significant role if a frequency measurement aimed at a relative accuracy of $10^{-13}$, which is typical of what is needed to investigate possible time variation of fundamental constants. For instance, a very sensitive probe for a possible time variation of the proton-electron mass ratio is the inversion frequency in ammonia. A new experiment is currently being set up, which aims at performing very high resolution spectroscopy in a Ramsey type interferometry experiment in an ammonia molecular fountain [7]. An accuracy of $10^{-13}-10^{-14}$ is expected on the measurement of the inversion frequency (of about $23.8 \mathrm{GHz}$ ) in the state $J=1, K=1$. Note that the hyperfine structure of ${ }^{15} \mathrm{ND}_{3}$ molecules has been recently measured with an accuracy of a few $10^{-9}$ in spectroscopy experiments using cold decelerated ammonia molecules [55]. The use of a molecular interferometer is expected to increase further the accuracy by several orders of magnitude. Therefore we evaluated the BBR-induced energy shift on both inversion levels involved in this experiment, which might be of importance since the levels considered are of different symmetries, i.e., are coupled to different sets of levels by the electric dipole interaction.

Microwave transitions, which have led to the ammonia maser [56], have been studied for decades in $\mathrm{NH}_{3}[57,58]$, as well as its rotation-inversion structure. We denote the lowest level involved in the inversion transition by $0^{-}, J=1, K=1$, the upper one being $0^{+}, J=1, K=1$, with the opposite parity. Both levels are coupled by electric dipole allowed transitions to all other rovibrational levels. Let us examine first the rotational radiative coupling: each of these levels is coupled to an inversion level of the $J=2, K=1$ rotation manifold via an electric dipole allowed transition. Because of the inversion-rotation structure (see for instance [59]), these two allowed transitions, around $39.7 \mathrm{~cm}^{-1}$, differ in frequency by the sum of the inversion splittings of the $J=1, K=1$ and $J=2, K=1$ states, i.e., by about $46.8 \mathrm{GHz}$. This implies that the levels $0^{-}, J=1, K=1$ and $0^{+}, J=1, K=1$ experience different BBR-induced shifts, given by eq.(8). Both BBR-induced shifts, on the order of $80 \mathrm{mHz}$ at room temperature, differ by about $5 \mathrm{mHz}$, which represents $210^{-13}$ times the inversion frequency between the $0^{-}, J=1, K=1$ and $0^{+}, J=1, K=1$ levels. Although not negligible in the absorption rate of BBR photons, the coupling to higher vibrational states via BBR photons only gives rise to light shifts lower than $10^{-14}$ times the inversion frequency (see for instance $[60,61]$ for electric dipole moment matrix elements). Note that the dipole moment matrix elements we used give an absorption rate compatible with the one calculated in ref. [18].

In conclusion, at room temperature the measured inversion frequency between the $0^{-}, J=1, K=1$ and $0^{+}, J=1, K=1$ levels is lowered by about $210^{-13}$ by BBR-induced energy shifts. We have also calculated that a room temperature variation of $5^{\circ} \mathrm{C}$ changes relatively the measured frequency by about $10^{-14}$. We did the same type of calculation on ${ }^{15} \mathrm{ND}_{3}$ and we draw the same conclusion, i.e., that the inversion 
frequency is lowered by about $210^{-13}$ by BBR-induced shifts. This should be considered carefully in a high-precision interferometry experiment aiming at measuring the inversion frequency of ammonia at an accuracy of $10^{-13}-10^{-14}$ and more generally in high-precision frequency measurements involving molecules.

\section{Conclusion}

We have performed calculations to evaluate the influence of the black body radiation on molecular systems in use in, or of relevance for high-precision measurements. Both absorption rates and induced energy shifts have been estimated. The BBR absorption could disturb the coherence of molecular interferometers if they are achieved in traps with heavy radicals like $\mathrm{YbF}, \mathrm{PbF}$ or $\mathrm{HgF}$, which hold promise in the search for the electron EDM. Alkali dimers should not suffer from the BBR absorption, except LiCs that survives in its ground state during one minute only, which might hamper further evaporative or sympathetic cooling. Much more critical is the case of $\mathrm{LiH}$, which is predicted to be pumped out of the prepared initial state $v=0$, $J=1$ in less than one second at room temperature and only in $3 \mathrm{~s}$ at liquid nitrogen temperature. Finally, we have shown that the BBR-induced shift on molecular level energies should be considered carefully in high-precision frequency measurements, like in the case of a molecular fountain of ammonia, which is currently being built to investigate the time variation of the proton-electron mass ratio.

\section{Acknowledgements}

Useful discussions with Christian Jungen are gratefully acknowledged. We are indebted to Mireille Aymar for providing dipole moment functions of alkali hydrides. This work has been supported by the "Institut Francilien de Recherche sur les Atomes Froids" (IFRAF) and by the "Agence National de la Recherche" (ANR grant NT05-2 41884).

\section{References}

[1] J. H. Christenson, J. W. Cronin, V. L. Fitch, and R. Turlay. Evidence for the $2 \pi$ decay of the $K_{2}^{0}$ meson. Phys. Rev. Lett, 13:138, 1964.

[2] M. A. Bouchiat and C. Bouchiat. Parity violation induced by weak neutral currents in atomic physics. J. Phys., 35:899, 1975.

[3] J. Guena, M. Lintz, and M. A. Bouchiat. Measurement of the parity violating 6S-7S transition amplitude in cesium achieved within $210^{-13}$ atomic-unit accuracy by stimulated-emission detection. Physical Review A (Atomic, Molecular, and Optical Physics), 71(4):042108, 2005.

[4] S. C. Bennett and C. E. Wieman. Measurement of the $6 S \rightarrow 7 S$ transition polarizability in atomic cesium and an improved test of the standard model. Phys. Rev. Lett., 82(12):2484-2487, 1999.

[5] E. Peik, B. Lipphardt, H. Schnatz, T. Schneider, C. Tamm, and S. G. Karshenboim. Limit on the present temporal variation of the fine structure constant. Phys. Rev. Lett., 93(17):170801, 2004.

[6] E. R. Hudson, H. J. Lewandowski, B. C. Sawyer, and J. Ye. Cold molecule spectroscopy for contraining the evolution of the fine structure constant. Phys. Rev. Lett, 96:143004, 2006.

[7] H. L. Bethlem. Private communication.

[8] M. V. Romalis, W. C. Griffith, J. P. Jacobs, and E. N. Fortson. New limit on the permanent electric dipole moment of ${ }^{199} \mathrm{Hg}$. Phys. Rev. Lett, 86:2505, 2001.

[9] J. R. Guest, N. D. Scielzo, I. Ahmad, K. Bailey, J. P. Greene, R. J. Holt, Z.-T. Lu, T. P. O'Connor, and D. H. Potterveld. Lasertrapping of Ra-225 and Ra-226 with repumping by room temperature blackbody radiation. physics/0701263, 2007.

[10] C. A. Baker, D. D. Doyle, P. Geltenbort, K. Green, M. G. D. van der Grinten, P. G. Harris, P. Iaydjiev, S. N. Ivanov, D. J. R. May, J. M. Pendlebury, J. D. Richardson, D. Shiers, and K. F. Smith. Improved experimental limit on the electric dipole moment of the neutron. Phys. Rev. Lett., 97(13):131801, 2006.

[11] B. C. Regan, E. D. Commins, C. J. Schmidt, and D. DeMille. New limit on the electron electric dipole moment. Phys. Rev. Lett., 88(7):071805, 2002.

[12] F. J. M. Farley, K. Jungmann, J. P. Miller, W. M. Morse, Y. F. Orlov, B. L. Roberts, Y. K. Semertzidis, A. Silenko, and E. J. Stephenson. New method of measuring electric dipole moments in storage rings. Phys. Rev. Lett., 93(5):052001, 2004.

[13] J. J. Hudson, B. E. Sauer, M. R. Tarbutt, and E. A. Hinds. Measurement of the electron electric dipole moment using YbF molecules. Phys. Rev. Lett, 89:023003, 2002.

[14] D. DeMille, F. Bay, S. Bickman, D. Kawall, D. Krause, S. E. Maxwell, and L. R. Hunter. Investigation of PbO as a system for measuring the electric dipole moment of the electron. Phys. Rev. A, 61(5):052507, 2000.

[15] T. F. Gallagher and W. E. Cooke. Interactions of blackbody radiation with atoms. Phys. Rev. Lett, 42:835, 1979.

[16] U. Hechtfischer, Z. Amitay, P. Forck, M. Lange, J. Linkemann, M. Schmitt, U. Schramm, D. Schwalm, R. Wester, D. Zajfman, and A. Wolf. Near-threshold photodissociation of cold $\mathrm{CH}^{+}$in a storage ring. Phys. Rev. Lett., 80(13):2809-2812, 1998.

[17] I. S. Vogelius, L. B. Madsen, and M. Drewsen. Blackbody-radiation assisted laser cooling of molecular ions. Phys. Rev. Lett., 89(17):173003, 2002. 
[18] S. Hoekstra, J. J. Gilijamse, B. Sartakov, N. Vanhaecke, L. Scharfenberg, S. Y.T. van de Meerakker, and G. Meijer. Optical pumping of trapped neutral molecules by blackbody radiation. Phys. Rev. Lett., 98(13):133001, 2007.

[19] E. J. Angstmann, V. A. Dzuba, and V. V. Flambaum. Frequency shift of the cesium clock transition due to blackbody radiation. Phys. Rev. Lett., 97:040802, 2006.

[20] K. Beloy, U.I. Safronova, and A. Derevianko. High-accuracy calculation of the blackbody radiation shift in the ${ }^{133}$ Cs primary frequency standard. Phys. Rev. Lett., 97:040801, 2006.

[21] S. G. Porsev and A. Derevianko. Multipolar theory of blackbody radiation shift of atomic energy levels and its implications for optical lattice clocks. Phys. Rev. A, 74:020502, 2006.

[22] M. Dolg, H. Stoll, and H. Preuss. Ab initio pseudopotential study of YbH and YbF. Chem. Phys., 165:21, 1992.

[23] W. Liu, M. Dolg, and L. Lemin. Fully relativistic density functional calculations of the ground and excited states of $\mathrm{Yb}, \mathrm{YbH}, \mathrm{YbF}$, and YbO. J. Chem. Phys., 108:2886, 1998.

[24] K. K. Das, I. D. Petsalakis, H.-P. Liebermannand A. B. Alekseyev, and R. J. Buenker. Ab initio spin-orbit CI calculations of the potential curves and radiative lifetimes of low-lying states of lead monofluoride. J. Chem. Phys., 116:608, 2002.

[25] S.R. Langhoff, C. W. Jr. Bauschlicher, H. Partridge, and R. Ahlrichs. Theoretical study of the dipole moments of selected alkalineearth halides. J. Chem. Phys., 84:5025, 1986.

[26] M. Arif, Ch. Jungen, and A. L. Roche. Binding energy and dipole moment of alkali halide molecules. J. Chem. Phys., 106:4102, 1996.

[27] Y. Y. Dmitriev, Y. G. Khait, M. G. Kozlov, L.N. Labzovsky, A. O. Mitrushenkov, A.V. Shtoff, and A.V. Titov. Calculation of the spin-rotational Hamiltonian including $\mathrm{P}$ - and $\mathrm{P}, \mathrm{T}$-odd weak interaction terms for $\mathrm{HgF}$ and $\mathrm{PbF}$ molecules. Physics Letters A, $167: 280,1992$.

[28] S. F. Rice, H. Martin, and R. W. Field. The electronic structure of the calcium monohalides. A ligand field approach. J. Chem. Phys., 82:5023, 1985.

[29] A. R. Allouche, G. Wannous, and M. Aubert-Frécon. A ligand-field approach for the low-lying states of Ca, Sr and Ba monohalides. Chem. Phys., 170:11, 1993.

[30] E. S. Rittner. Binding energy and dipole moment of alkali halide molecules. J. Chem. Phys., 19:1030, 1951.

[31] T. Törring, W. E. Enrst, and S. Kindt. Dipole moments and potential energies of the alkaline earth monohalides from an ionic model. J. Chem. Phys., 81:4614, 1984.

[32] G. H. F. Diercksen and A. J. Sadlej. Electric properties of negative ions. Dipole hyperpolarizability of the fluoride ion. Mol. Phys., 47:33, 1982.

[33] S. A. Kucharski, Y. S. Lee, G. D. Purvis III, and R. J. Bartlett. Dipole polarizability of the fluoride ion with many-body methods. Phys. Rev. A, 29:1619, 1984.

[34] W. E. Enrst, Kändler, and T. Törring. Hyperfine structure and electric dipole moment of $\mathrm{BaF} \mathrm{X}^{2} \Sigma^{+}$. Chem. Phys. Lett., 84:4769, 1986.

[35] E. J. Angstmann, V. A. Dzuba, and V. V. Flambaum. Frequency shift of hyperfine transitions due to the blackbody radiation. Phys. Rev. A, 74:023405, 2006.

[36] T. Törring, W. E. Enrst, and J. Kändler. Energies and electric dipole moments of the low electronic states of the alkaline earth monohalides from an electrostatic polarization model. J. Chem. Phys., 90:4927, 1989.

[37] M. Aymar and O. Dulieu. Calculation of accurate permanent dipole moments of the lowest ${ }^{1,3} \Sigma^{+}$states of heteronuclear alkali dimers using extended basis sets. J. Chem. Phys., 122:204302, 2005.

[38] M. Aymar. Private communication.

[39] A. Borin and F. Ornellas. The lowest triplet and singlet electronic states of the molecule SO. Chem. Phys., 247:351, 1999.

[40] J. Weiner. Advances in ultracold collisions: experimentation and theory. Advances in Atomic, Molecular and Optical Physics, 35:45, 1995.

[41] M. A. Baranov, M. S. Mar'enko, V. S. Rychkov, and G. V. Shlyapnikov. Superfluid pairing in a polarized dipolar fermi gas. Physical Review A (Atomic, Molecular, and Optical Physics), 66(1):013606, 2002.

[42] D. DeMille. Quantum computation with trapped polar molecules. Phys. Rev. Lett., 88(6):067901, 2002.

[43] J. M. Sage, S. Sainis, T. Bergeman, and D. DeMille. Optical production of ultracold polar molecules. Phys. Rev. Lett., 94(20):203001, 2005.

[44] M. Mudrich, S. Kraft, J. Lange, A. Mosk, M. Weidemüller, and E. Tiesinga. Hyperfine-changing collisions in an optically trapped gas of ultracold cesium and lithium. Physical Review A (Atomic, Molecular, and Optical Physics), 70(6):062712, 2004.

[45] M. W. Mancini, G. D. Telles, A. R. L. Caires, V. S. Bagnato, and L. G. Marcassa. Observation of ultracold ground-state heteronuclear molecules. Phys. Rev. Lett., 92(13):133203, 2004.

[46] S. K. Tokunaga, J. O. Stack, J. J. Hudson, B. E. Sauer, E. A. Hinds, and M. R. Tarbutt. A supersonic beam of cold lithium hydride molecules. physics/0612163, 2006.

[47] S.Y.T. van de Meerakker, N. Vanhaecke, and G. Meijer. Deceleration and trapping of OH radicals. Ann. Rev. Phys. Chem., 57:159, 2006.

[48] V. Kokoouline, O. Dulieu, R. Kosloff, and F. Masnou-Seeuws. Mapped Fourier methods for long-range molecules: application to perturbations in the $\mathrm{Rb}_{2}\left(0_{u}^{+}\right)$photoassociation spectrum. J. Chem. Phys., 110:9865, 1999.

[49] S. Jung, E. Tiemann, and C. Lisdat. Cold atoms and molecules from fragmentation of decelerated $\mathrm{SO}_{2}$. Physical Review A (Atomic, Molecular, and Optical Physics), 74(4):040701, 2006.

[50] S. Kotochigova, P. S. Julienne, and E. Tiesinga. Ab initio calculation of the KRb dipole moments. Phys. Rev. A, 68:022501, 2003.

[51] S. Kotochigova and E. Tiesinga. Ab initio relativistic calculation of the RbCs molecule. J. Chem. Phys., 123:174304, 2005.

[52] C. Cohen-Tannoudji, J. Dupont-Roc, and G. Grynberg. Processus d'interaction entre photons et atomes. EDP Sciences, 1988.

[53] W. M. Itanov, L. L. Lewis, and D. J. Wineland. Shift of ${ }^{2} S_{1 / 2}$ hyperfine splittings due to blackbody radiation. Phys. Rev. A, 25:1233, 1982.

[54] A. Donges. The coherence length of black-body radiation. Eur. J. Phys., 19:245, 1998.

[55] J van Veldhoven, J Küpper, H. L. Bethlem, B. Sartakov, A. J. A. van Roij, and G Meijer. Decelerated molecular beams for highresolution spectroscopy. Eur. Phys. J. D, 31:337, 2004.

[56] J. P. Gordon, H. J. Zeiger, and C. H. Townes. The maser new type of microwave amplifier, frequency standard, and spectrometer. Phys. Rev., 99(4):1264-1274, 1955.

[57] W. E. Good and D. K. Coles. Microwave absorption frequencies of ${ }^{14} \mathrm{NH}_{3}$ and ${ }^{15} \mathrm{NH}_{3}$. Phys. Rev., 71(6):383-384, 1947.

[58] C. C. Costain. An empirical formula for the microwave spectrum of ammonia. Phys. Rev., 82(1):108, 1951.

[59] C. H. Townes and A. L. Schawlow. Microwave spectroscopy. Dover Publications, Inc., 1975.

[60] S. N. Yurchenko, M. Carvajal, H. Lin, J. Zheng, W. Thiel, and P. Jensen. Dipole moment and rovibrational intensities in the electronic ground state of $\mathrm{NH}_{3}$ : Bridging the gap between ab initio theory and spectroscopic experiment. J. Chem. Phys., 122:104317, 2005. 


\section{$\begin{array}{rrrr}\text { May 7, } 2007 & 15: 5 & \text { Molecular Physics } & \text { resubmitted } \\ \text { Page } \mathbf{1 1} \text { of } \mathbf{1 3 5} & & & \text { Molecular Physics }\end{array}$}

Precision measurements with polar molecules...

1

2

3 4

5

6

7

8

9

10

11

12

13

14

15

16

17

18

19

20

21

22

23

24

25

26

27

28

29

30

31

32

33

34

35

36

37

38

39

40

41

42

43

44

45

46

47

48

49

50

51

52

53

54

55

56

57

58

59

60

[61] S. N. Yurchenko, W. Thiel, M. Carvajal, H. Lin, and P. Jensen. Rotation-vibration motion of pyramidal XY 3 molecules described in the Eckart frame: The calculation of intensities with application to $\mathrm{NH}_{3}$. Advances in Quantum Chemistry, 48:209, 2005. 


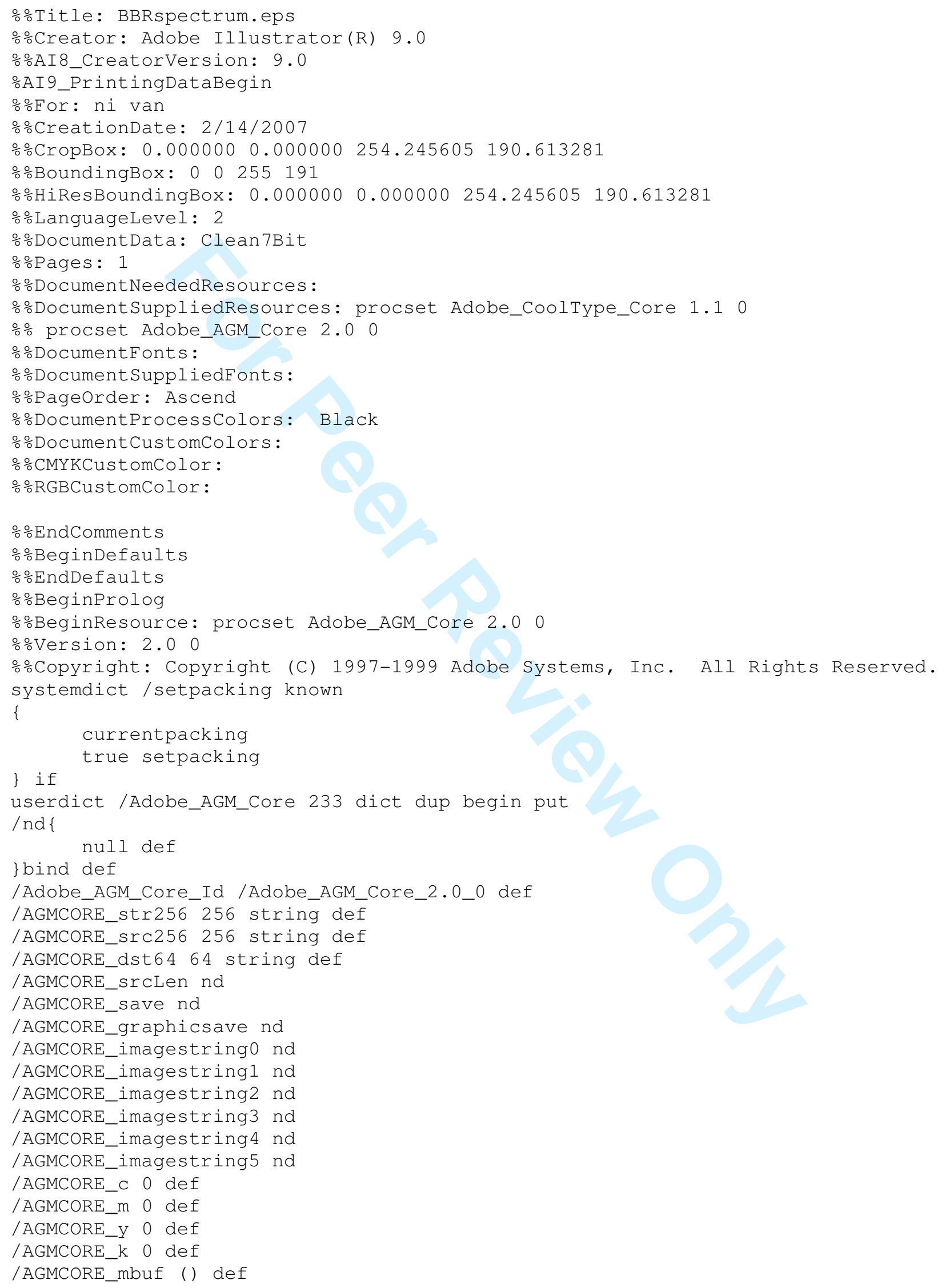

URL: http://mc.manuscriptcentral.com/tandf/tmph 
/AGMCORE_ybuf () def /AGMCORE_kbuf () def /AGMCORE_gbuf () def /AGMCORE_bbuf () def /AGMCORE_cmykbuf 4 array def 


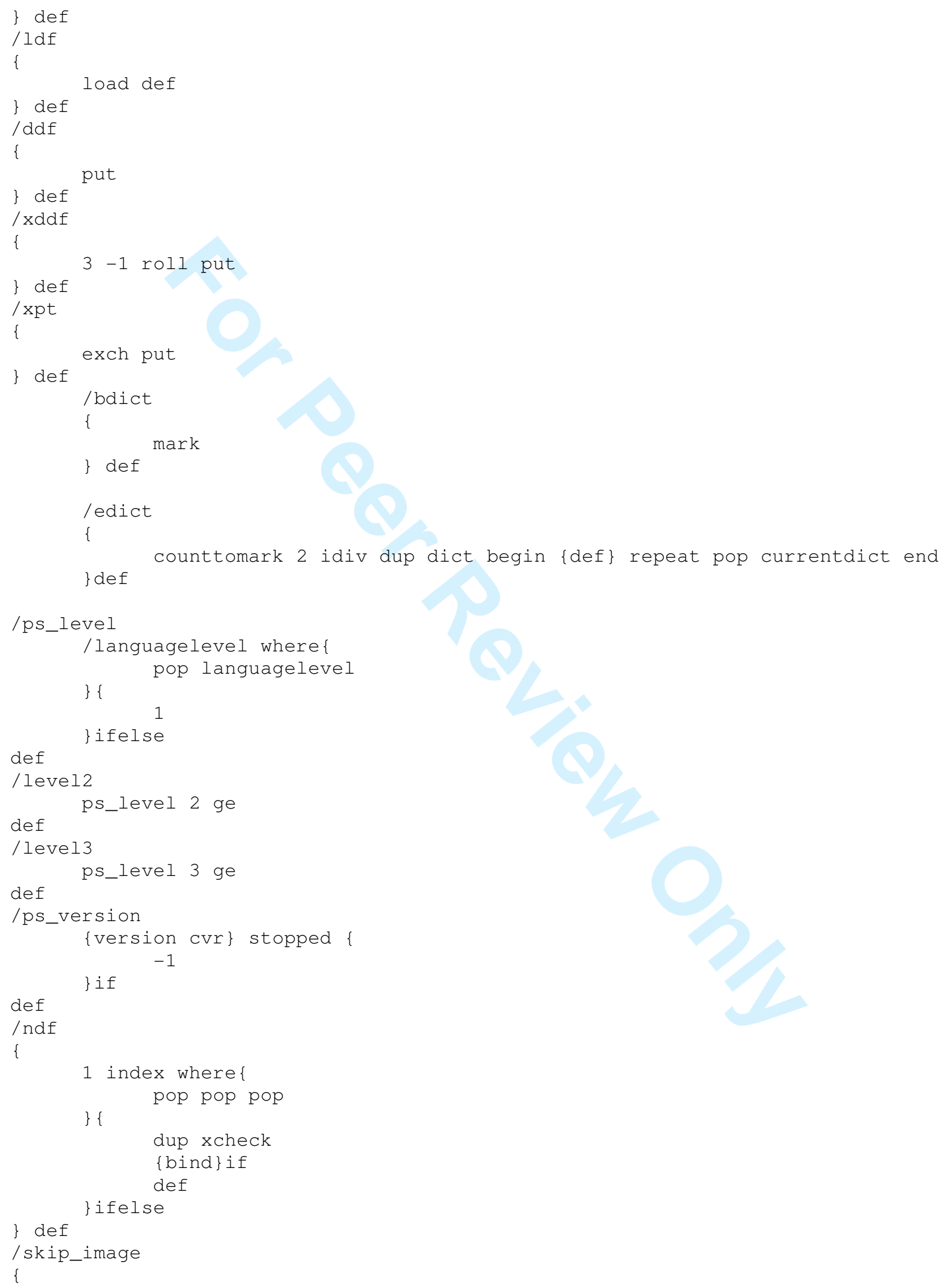


\} def 


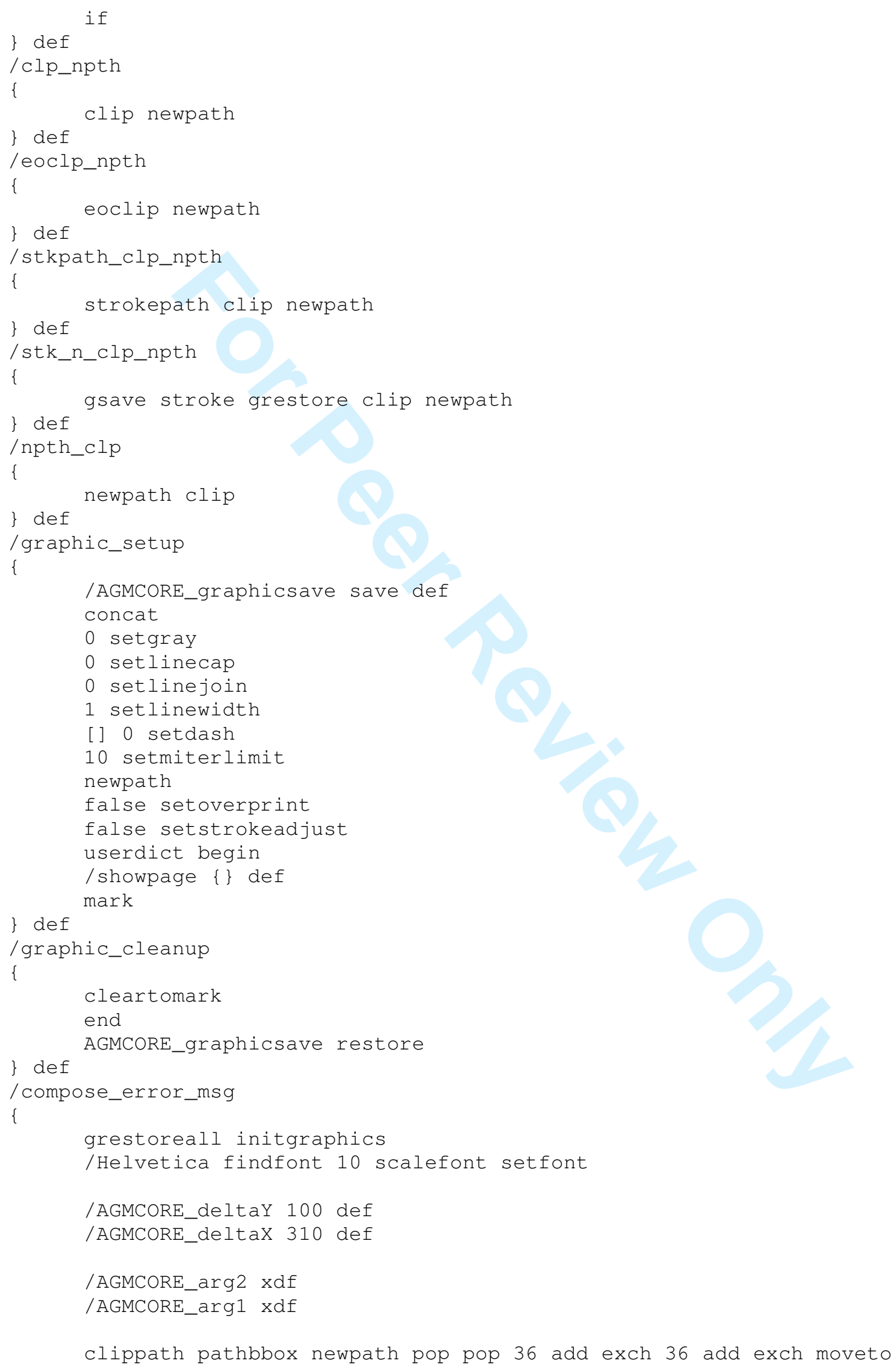


0 AGMCORE_deltaY rlineto AGMCORE_deltaX 0 rlineto

O AGMCORE_deltaY neg rlineto AGMCORE_deltaX neg 0 rlineto closepath

0 AGMCORE_\&setgray

gsave 1 AGMCORE_\&setgray fill grestore

1 setlinewidth gsave stroke grestore

currentpoint AGMCORE_deltaY 15 sub add exch 8 add exch moveto

/AGMCORE_deltaY 12 def

/AGMCORE_tmp 0 def

AGMCORE_err_strings exch get

\{

dup 32 eq

\{

pop

AGMCORE_str256 0 AGMCORE_tmp getinterval

dup (.) ne AGMCORE_argl 0 lt and

i

pop

\}

\{

stringwidth pop currentpoint pop add AGMCORE_deltaX 28

add gt

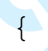

01 AGMCORE_str256 length 1 sub

\{

AGMCORE_str256 exch 0 put

\} for

/AGMCORE_tmp 0 def

\}

dup 94 eq

\{

pop

AGMCORE_arg1 0 ge

\{

AGMCORE_arg1 AGMCORE_str256 CVS

dup /AGMCORE_tmp exch length def

AGMCORE_str256 exch 0 exch putinterval

AGMCORE_str256 0 AGMCORE_tmp getinterval

AGMCORE_deltaX 28 add gt stringwidth pop currentpoint pop add

moveto
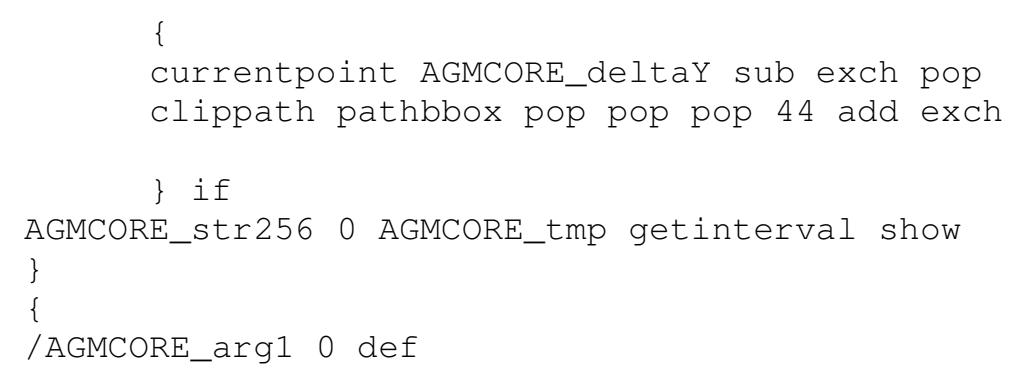

URL: http://mc.manuscriptcentral.com/tandf/tmph 
\} ifelse

01 AGMCORE_str256 length 1 sub

\{

AGMCORE_str256 exch 0 put

\} for

/AGMCORE_tmp 0 def

AGMCORE_arg1 0 ne

\{

/AGMCORE_arg1 AGMCORE_arg2 def

\} if

\}

AGMCORE_str256 exch AGMCORE_tmp exch put

/AGMCORE_tmp AGMCORE_tmp 1 add def

\}ifelse

forall

\} bdf

level2 \{

/AGMCORE_map_reserved_ink_name

\{

dup type / stringtype eq dup /Red eqf

pop (_Red_)

\}\{

dup /Green eq

pop (_Green_)

\}\{

dup /Blue eq\{

pop (_Blue_)

\}\{

dup /Cyan eq\{

pop (_Cyan_)

\}\{

dup /Magenta eq\{

pop (_Magenta_)

\}\{

dup /Yellow eq pop (_Yellow_)

\}\{

dup / Black eq\{

pop (_Black_)

\} \{ dup / eq \{

45

46

47

48

49

50

51

52

53

54

55

56

57

58

59

60

URL: http://mc.manuscriptcentral.com/tandf/tmph 
URL: http://mc.manuscriptcentral.com/tandf/tmph 


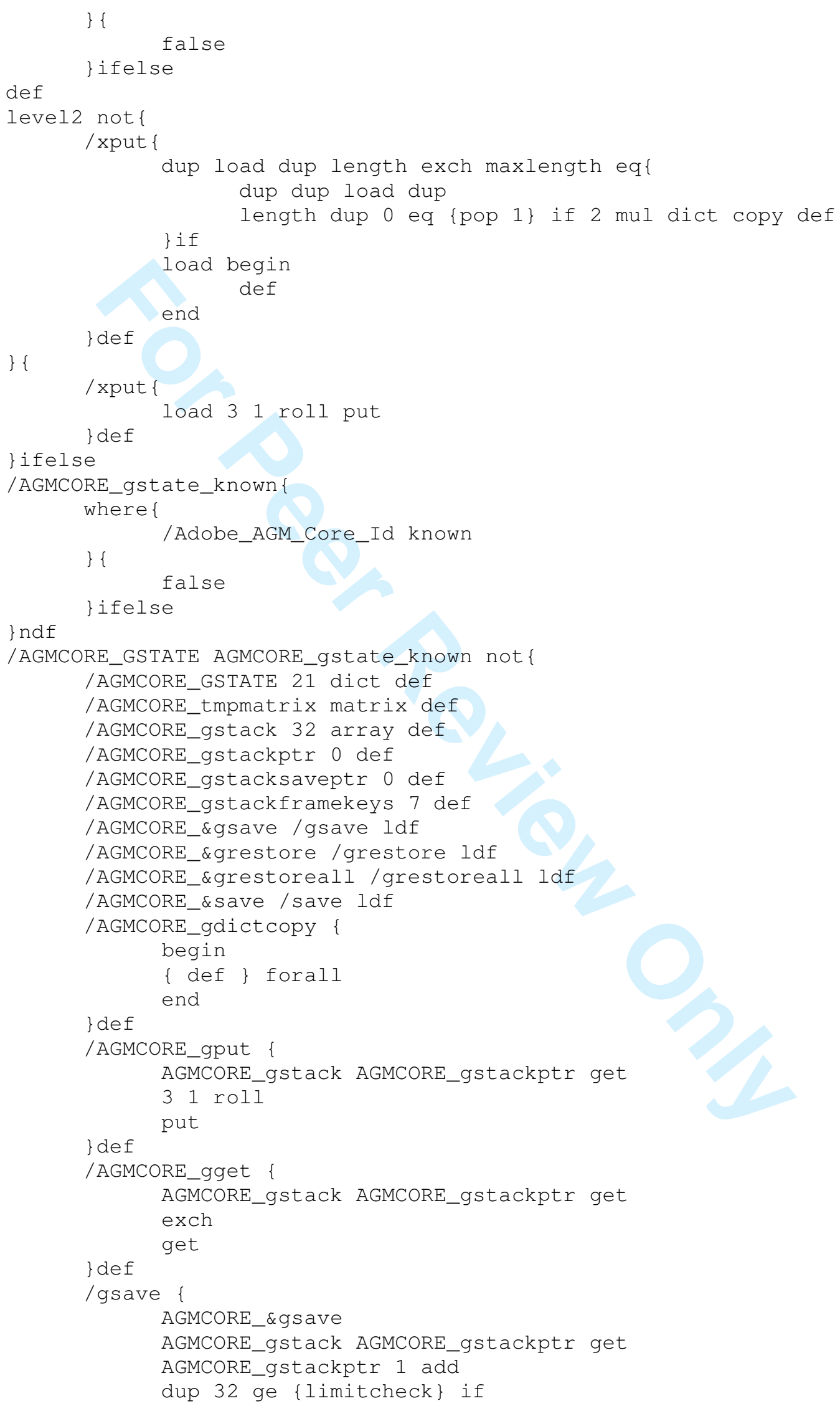

URL: http://mc.manuscriptcentral.com/tandf/tmph 
/AGMCORE_CCimage_exists /customcolorimage where \{pop true\}\{false\} ifelse

def

/ currentcmykcolor

\{

\} ndf

/currentcmykcolor AGMCORE_gget aload pop

/setoverprint

\{

\} ndf

pop

/ currentoverprint

\{

false

\} ndf

/AGMCORE_deviceDPI 72 o matrix defaultmatrix dtransform dup mul exch dup mul add sqrt def

/AGMCORE_cyan_plate 1000 test_cmyk_color_plate def

/AGMCORE_magenta_plate 0100 test_cmyk_color_plate def

/AGMCORE_yellow_plate 0010 test_cmyk_color_plate def

/AGMCORE_black_plate 0001 test_cmyk_color_plate def

/AGMCORE_plate_ndx

AGMCORE_cyan_plate\{

0

\}\{

AGMCORE_magenta_plate\{

1

\}\{

AGMCORE_yellow_plate\{

2

\}\{

AGMCORE_black_plate\{

3

\} \{

4

\}ifelse

\}ifelse

\}ifelse

def

/AGMCORE_composite_job

AGMCORE_cyan_plate AGMCORE_magenta_plate and AGMCORE_yellow_plate

and AGMCORE_black_plate and def

/AGMCORE_producing_seps AGMCORE_composite_job not AGMCORE_in_rip_sep or

def

/AGMCORE_host_sep AGMCORE_producing_seps AGMCORE_in_rip_sep not and def

/AGM_preserve_spots

/AGM_preserve_spots where\{

\}\{

pop AGM_preserve_spots

systemdict/setdistillerparams known product (Adobe PostScript

Parser) ne and AGMCORE_producing_seps or \}ifelse

def

URL: http://mc.manuscriptcentral.com/tandf/tmph 
AGMCORE_host_sep AGMCORE_will_host_separate not and \{ /AGMCORE_Cur_err /AGMCORE_Color_space_onhost_seps def AGMCORE_color_space_onhost_seps

\}if

/AGMCORE_avoid_L2_sep_space version cvr 2012 lt level2 and 
\{dup AGMCORE_c mul exch dup AGMCORE_m mul exch dup AGMCORE_y mul exch AGMCORE_k mul $\}$

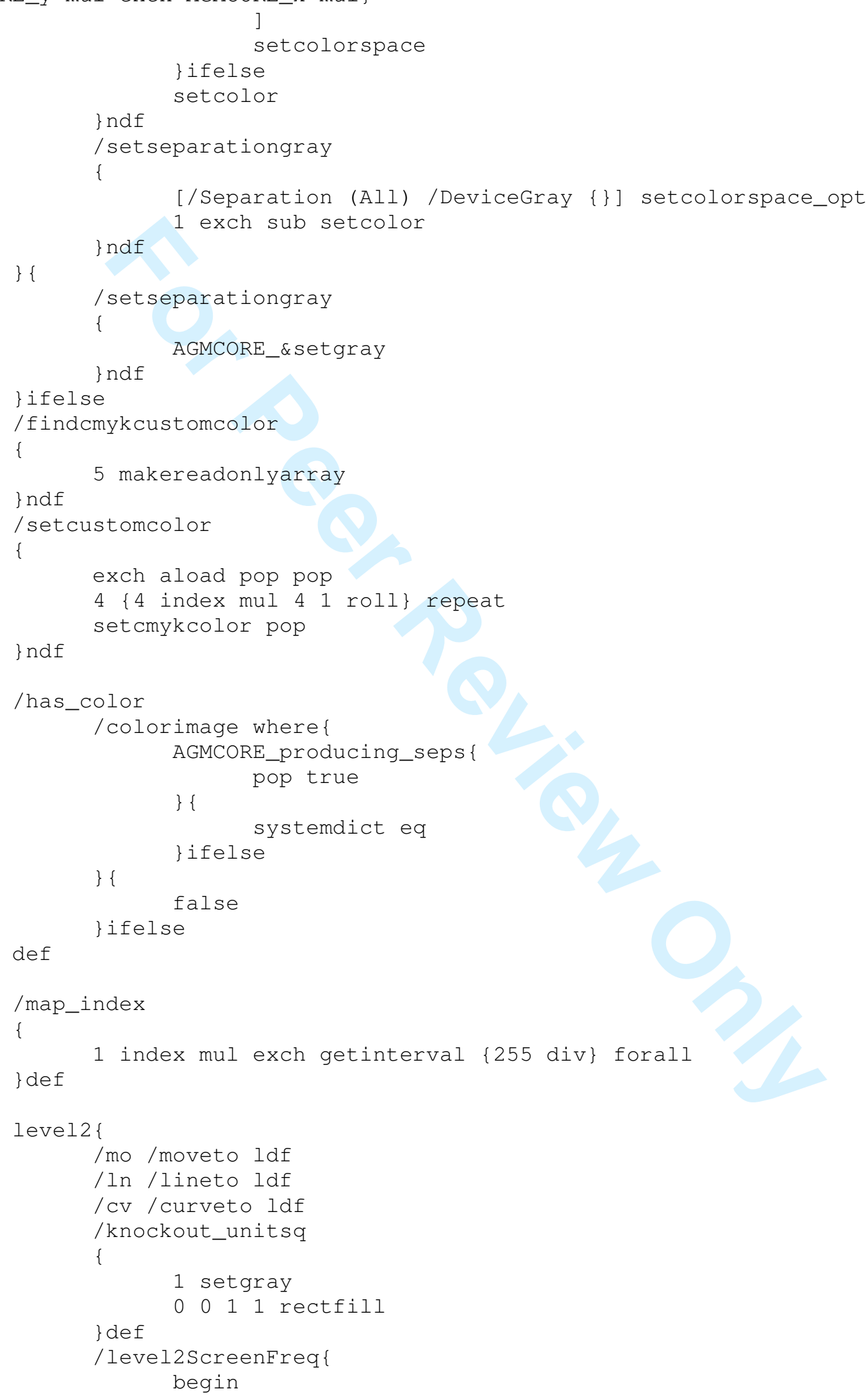

URL: http://mc.manuscriptcentral.com/tandf/tmph 
roll put

60

HalftoneType 1 eq

pop Frequency

\}if

HalftoneType 2 eq\{ pop GrayFrequency

\}if

HalftoneType 5 eq pop Default level2ScreenFreq

URL: http://mc.manuscriptcentral.com/tandf/tmph 
end

\} ndf

/set_gstate

\{

AGMCORE_GSTATE begin

AGMCORE_GSTATE_Ctm setmatrix

AGMCORE_GSTATE_Clr_spc setcolorspace

AGMCORE_GSTATE_clr_indx \{AGMCORE_GSTATE_clr_comps

AGMCORE_GSTATE_Clr_indx 1 sub get

/AGMCORE_GSTATE_Clr_indx AGMCORE_GSTATE_Clr_indx 1 sub def

repeat setcolor

AGMCORE_GSTATE_fnt setfont

AGMCORE_GSTATE_lw setlinewidth

AGMCORE_GSTATE_lC setlinecap

AGMCORE_GSTATE_lj setlinejoin

AGMCORE_GSTATE_ml setmiterlimit

AGMCORE_GSTATE_da AGMCORE_GSTATE_do setdash

AGMCORE_GSTATE_sa setstrokeadjust

AGMCORE_GSTATE_Clr_rnd setcolorrendering

AGMCORE_GSTATE_op setoverprint

AGMCORE_GSTATE_bg CVx setblackgeneration

AGMCORE_GSTATE_uCr CVX setunderColorremoval

AGMCORE_GSTATE_r_xfer CVx AGMCORE_GSTATE_g_xfer CVx

AGMCORE_GSTATE_b_xfer CVx

AGMCORE_GSTATE_gy_xfer CVx setcolortransfer

AGMCORE_GSTATE_ht /HalftoneType get dup 9 eq exch 100 eq or \{

currenthalftone/HalftoneType get AGMCORE_GSTATE_ht

/HalftoneType get ne

\{

cleartomark mark AGMCORE_GSTATE_ht \{sethalftone\} stopped

\} if

\}\{

AGMCORE_GSTATE_ht sethalftone

\} ifelse

AGMCORE_GSTATE_flt setflat

end

\} ndf

AGMCORE_producing_seps not \{

/setcolorspace where

/Adobe_AGM_Core_Id known not

\}\{

true

\}ifelse

\{

/ setcolorspace

\{

eq or \{

dup type dup /arraytype eq exch /packedarraytype

dup 0 get dup / Separation eqf

pop

[ exch \{\} forall]

URL: http://mc.manuscriptcentral.com/tandf/tmph 
dup dup 1 get

AGMCORE_map_reserved_ink_name 1 exch put

URL: http://mc.manuscriptcentral.com/tandf/tmph 


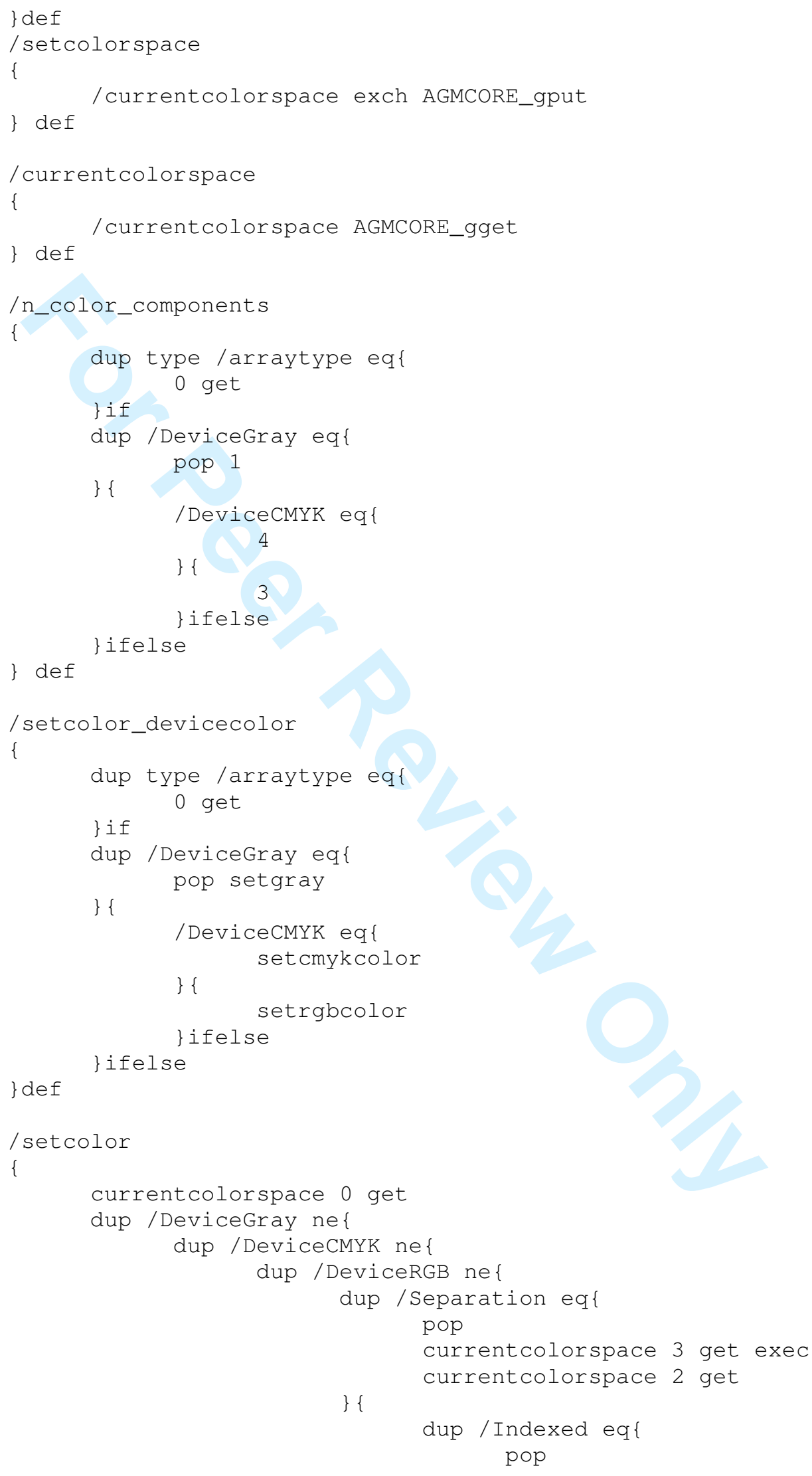

URL: http://mc.manuscriptcentral.com/tandf/tmph 


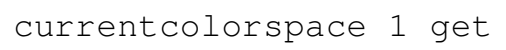

$$
\text { bind }
$$

URL: http://mc.manuscriptcentral.com/tandf/tmph 


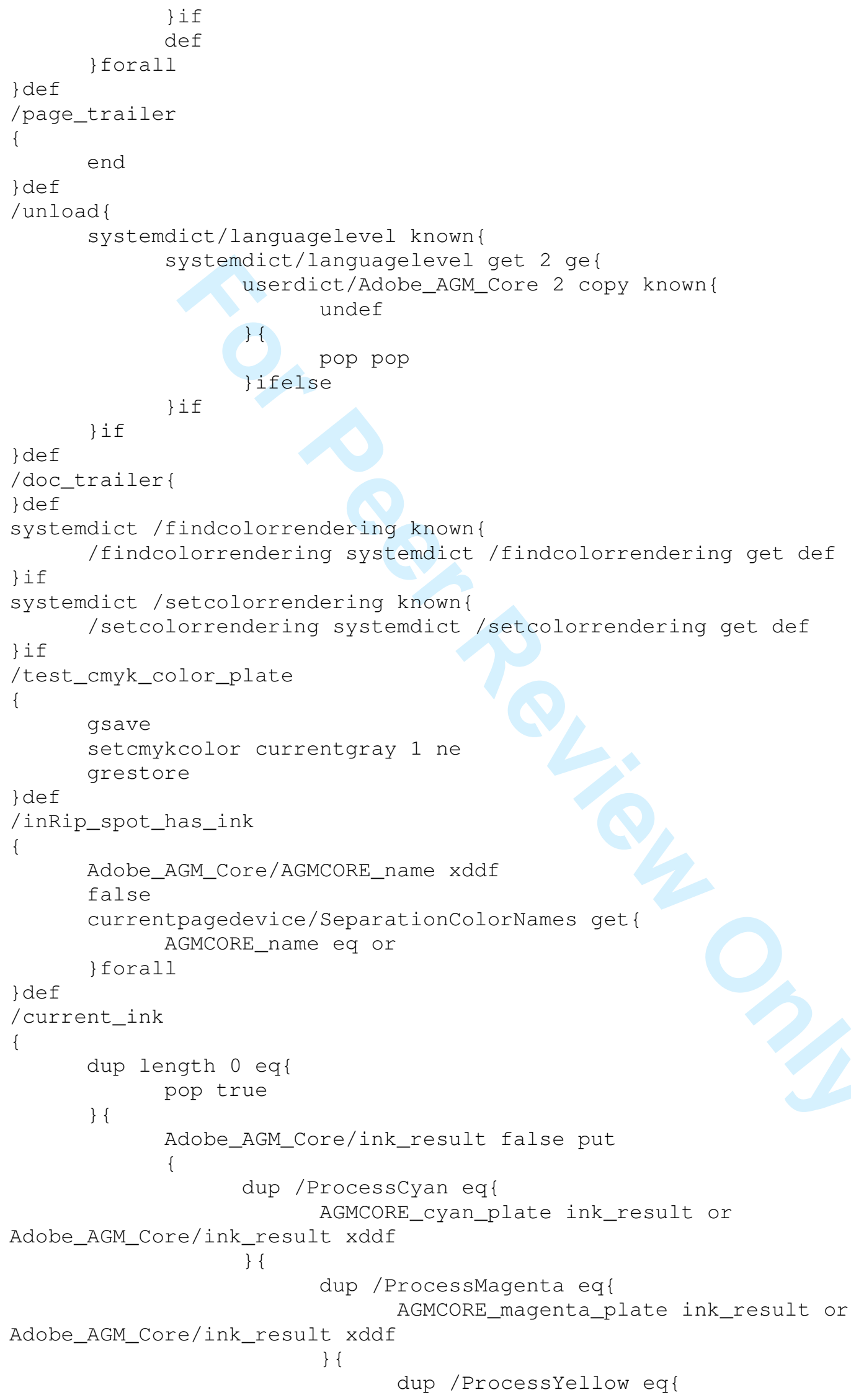

URL: http://mc.manuscriptcentral.com/tandf/tmph 
Adobe_AGM_Core/ink_result xddf

AGMCORE_yellow_plate ink_result or

\}\{

dup /ProcessBlack eq \{

AGMCORE_black_plate ink_result or

Adobe_AGM_Core/ink_result xddf

\}\{

dup null eqf

Adobe_AGM_Core/ink_result xddf

dup /sep_colorspace_dict AGMCORE_gget

pop false ink_result or 


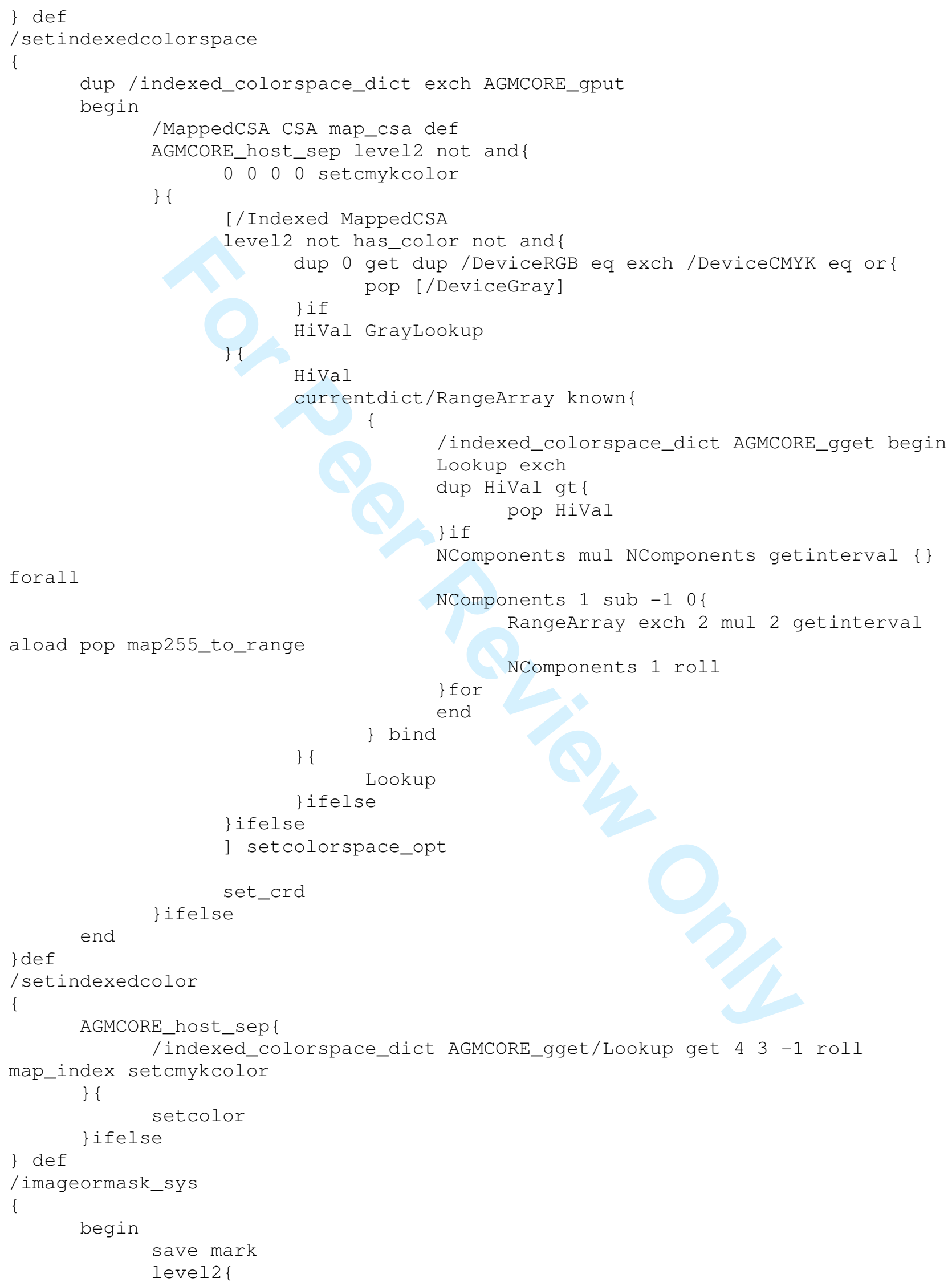

URL: http://mc.manuscriptcentral.com/tandf/tmph 
1

2

3

4

5

6

7

8

9

10

11

12

13

14

15

16

17

18

19

20

21

22

23

24

25

26

27

28

29

30

31

32

33

34

35

36

37

38

39

40

41

42

43

44

45

46

47

48

49

50

51

52

53

54

55

56

57

58

59

60

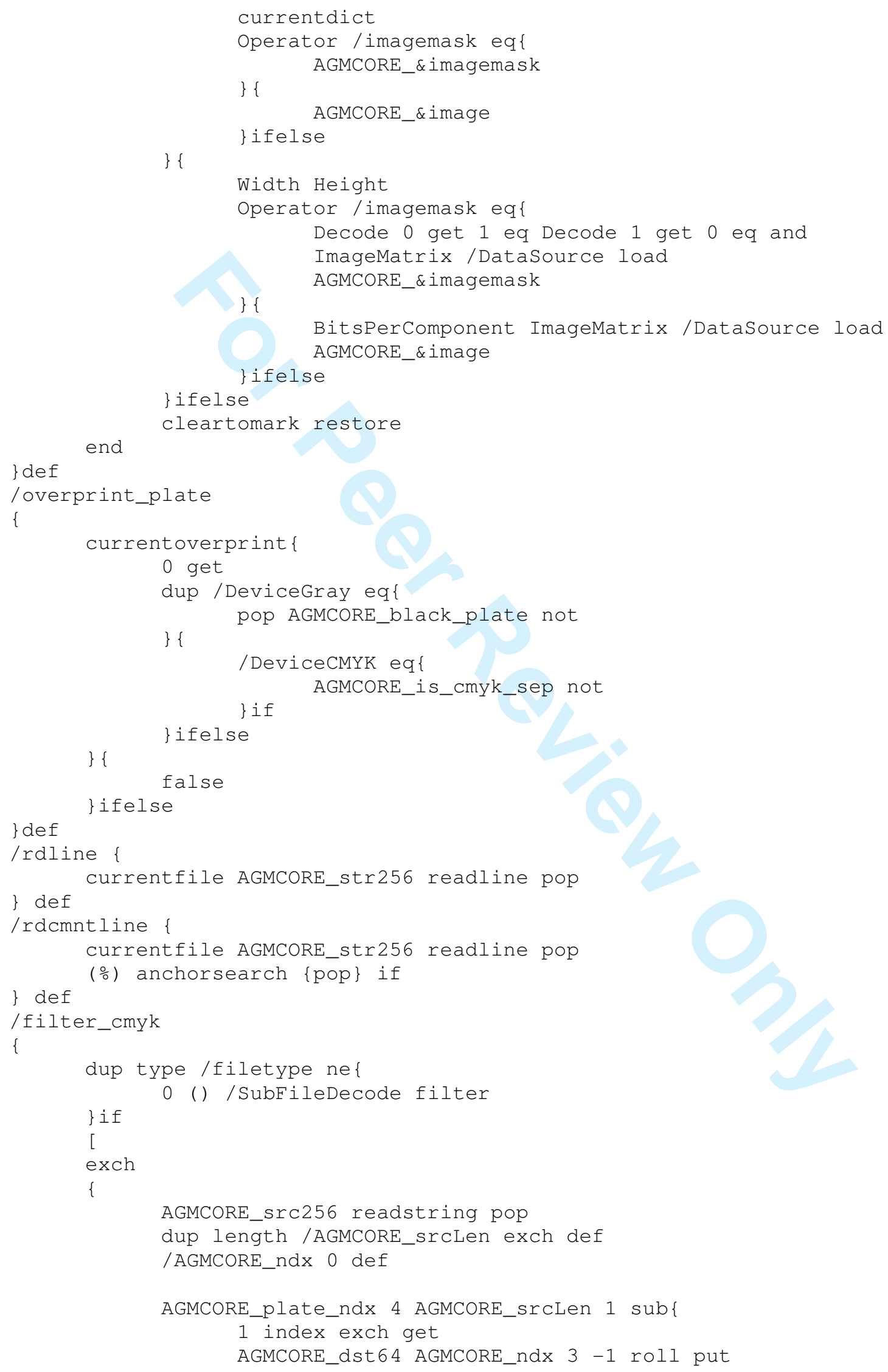

URL: http://mc.manuscriptcentral.com/tandf/tmph 


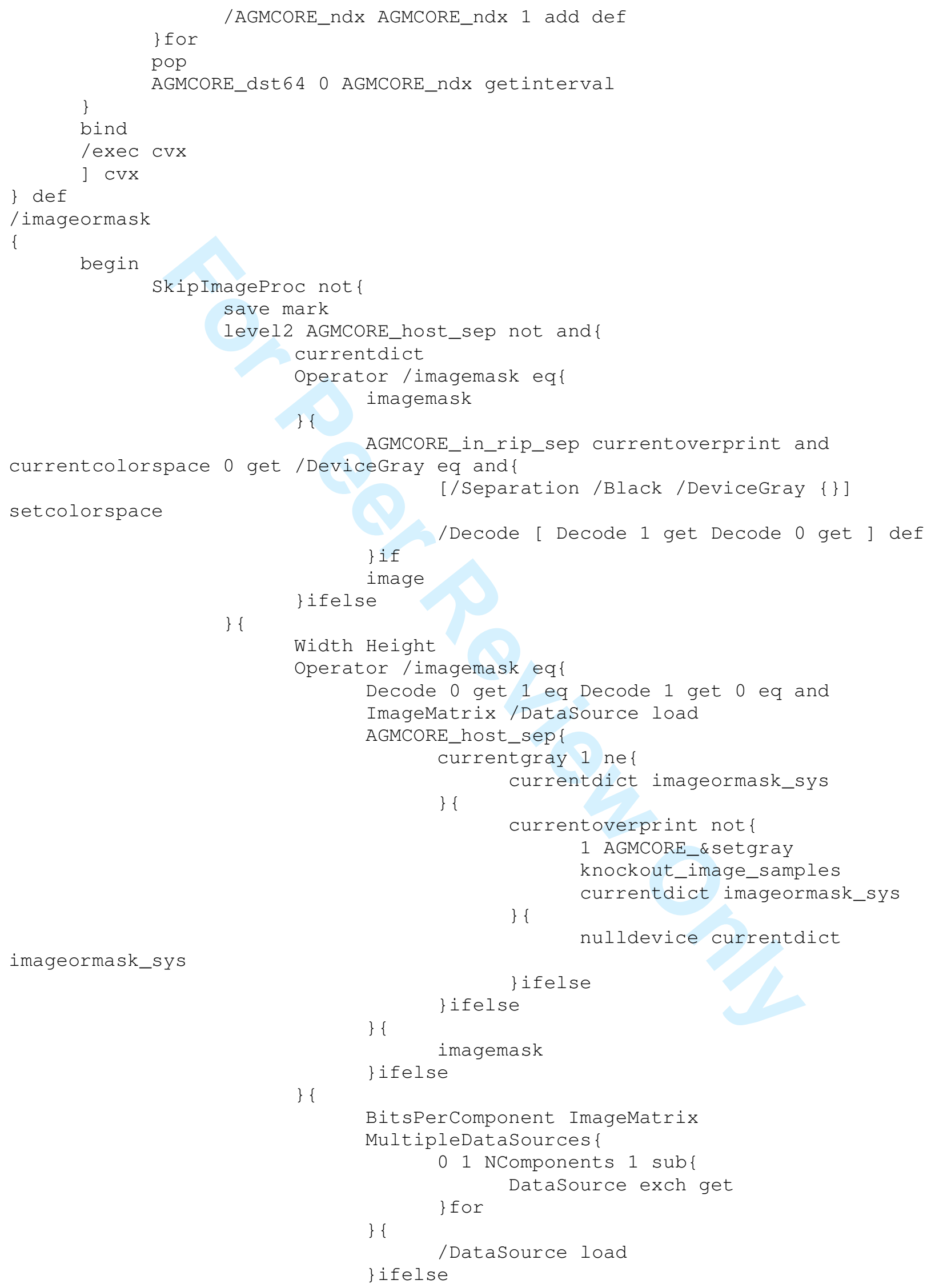

URL: http://mc.manuscriptcentral.com/tandf/tmph 


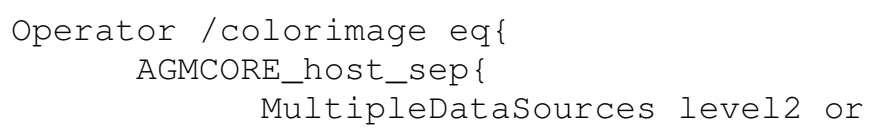


not \{

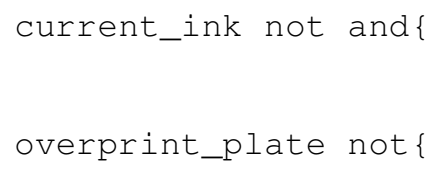


and \{

addprocs settransfer 


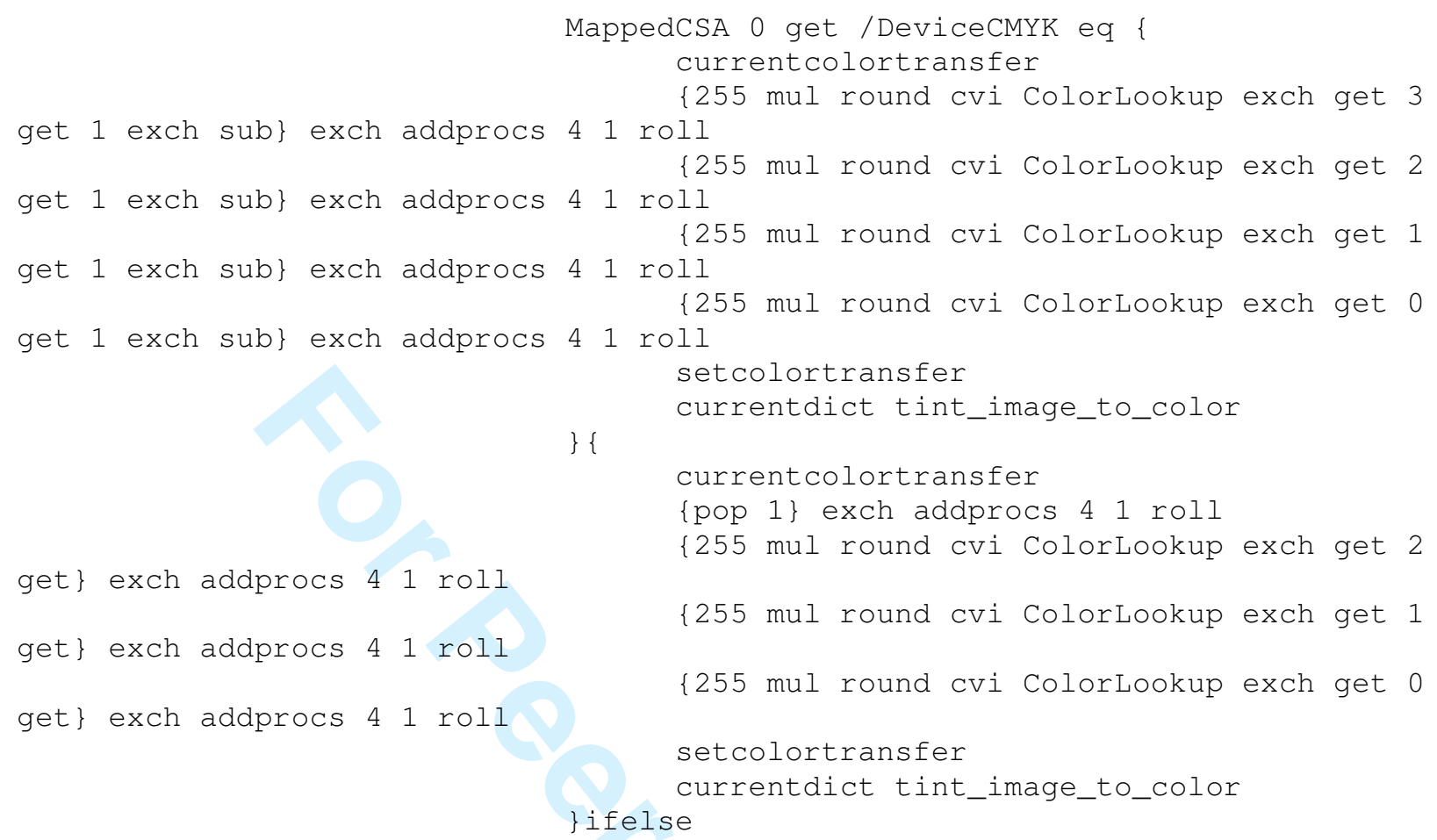




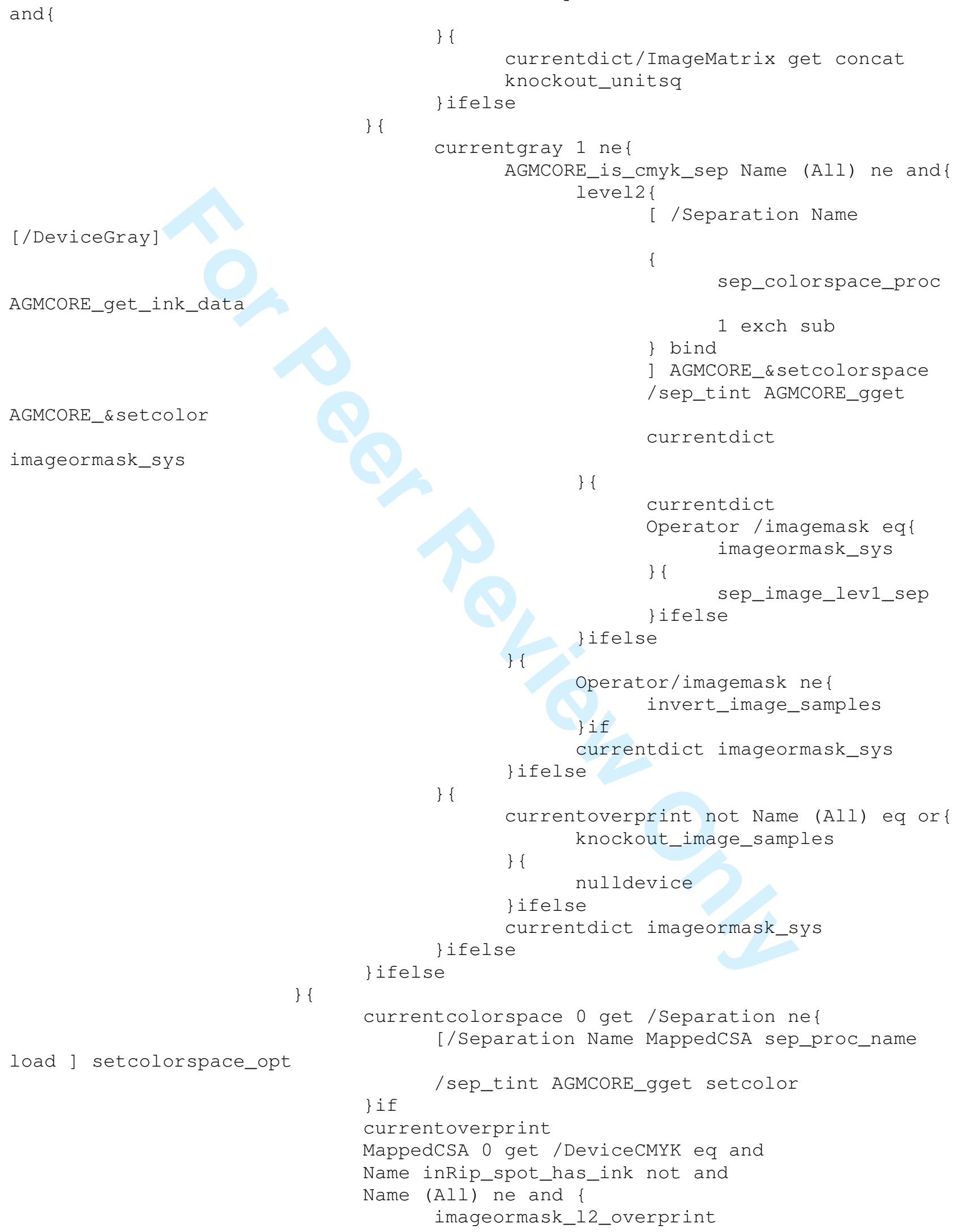

URL: http://mc.manuscriptcentral.com/tandf/tmph 
6

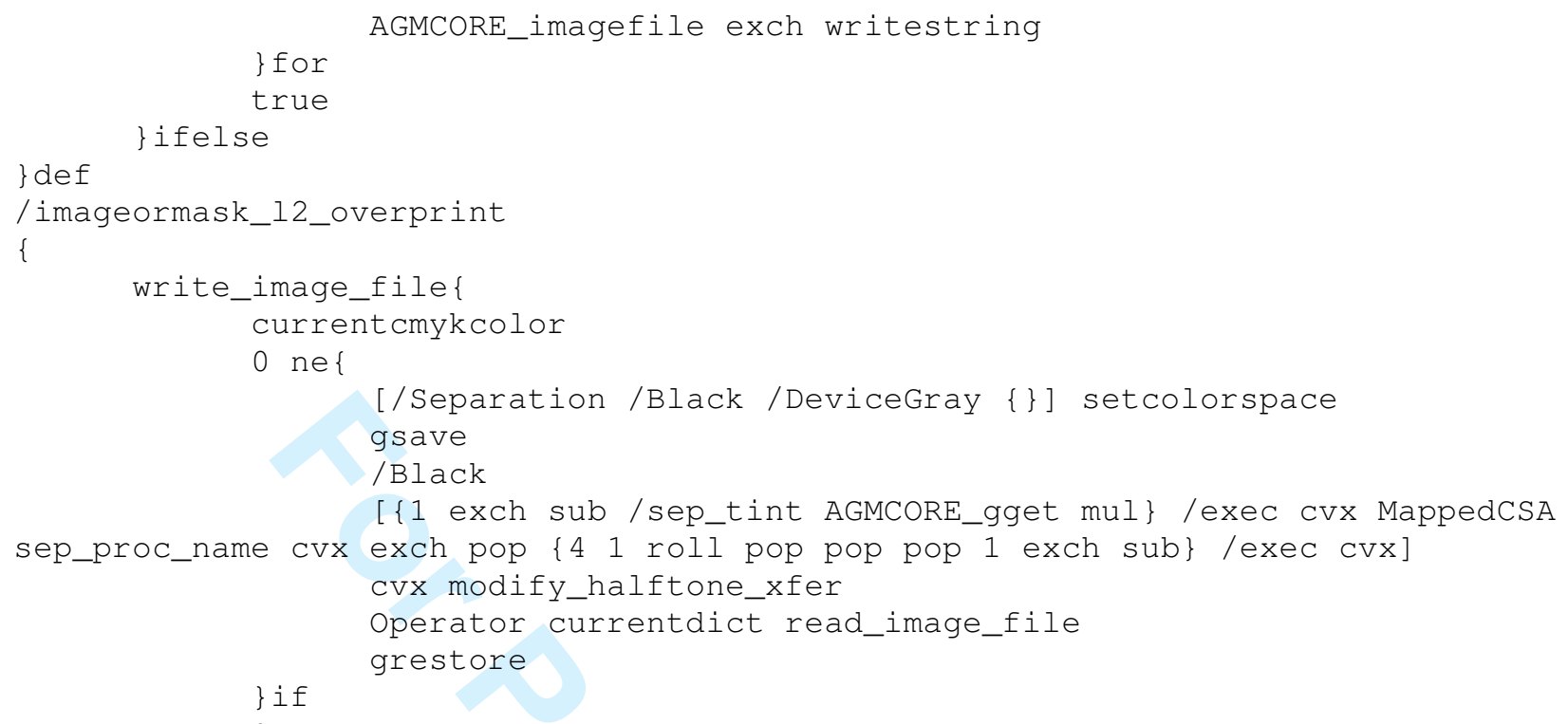

for

true 
1

2

3

4

5

6

7

8

9

10

11

12

13

14

15

16

17

18

19

20

21

22

23

24

25

26

27

28

29

30

31

32

33

34

35

36

37

38

39

40

41

42

43

44

45

46

47

48

49

50

51

52

53

54

55

56

57

58

59

60

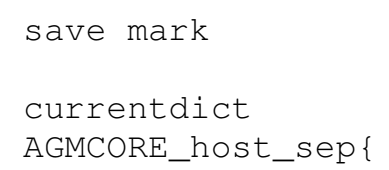

URL: http://mc.manuscriptcentral.com/tandf/tmph 
MappedCSA 0 get /DeviceGray eq \{

$\{$ HiVal mul round cvi Lookup exch get HiVal div\}

currenttransfer addprocs settransfer

imageormask

MappedCSA 0 get /DeviceCMYK eq \{

currentcolortransfer

\{4 mul HiVal mul round CVi 3 add Lookup exch get

HiVal div 1 exch sub\} exch addprocs 41 roll

\{4 mul HiVal mul round cvi 2 add Lookup exch get

HiVal div 1 exch sub\} exch addprocs 41 roll

\{4 mul HiVal mul round CVi 1 add Lookup exch get HiVal div 1 exch sub\} exch addprocs 41 roll

\{4 mul HiVal mul round cvi Lookup exch get

HiVal div 1 exch sub\} exch addprocs 41 roll

setcolortransfer

tint_image_to_color

\}\{

currentcolortransfer

\{pop 1 \} exch addprocs 41 roll

\{3 mul Hival mul round cvi 2 add Lookup exch get

HiVal div\} exch addprocs 41 roll

\{3 mul HiVal mul round cvi 1 add Lookup exch get HiVal div\} exch addprocs 41 roll

\{3 mul HiVal mul round cvi Lookup exch get

HiVal div exch addprocs 41 roll

setcolortransfer

\}ifelse

tint_image_to_color

30

31

32

33

34

35

36

37

38

39

40

41

42

43

44

45

46

47

48

49

50

51

52

53

54

55

56

end end

\} def

/indexed_image_lev1_sep

\{

/indexed_colorspace_dict AGMCORE_gget begin

begin

sub\}

\{4 mul HiVal mul round cvi Lookup exch get HiVal div 1 exch \{4 mul HiVal mul round cvi 1 add Lookup exch get HiVal div 1 exch

sub\}

sub\}

sub\}

\{4 mul HiVal mul round cvi 2 add Lookup exch get HiVal div 1 exch

\{4 mul HiVal mul round cvi 3 add Lookup exch get HiVal div 1 exch

AGMCORE_get_ink_data currenttransfer addprocs settransfer

currentdict imageormask_sys

end end

\} def

/indexed_image_lev2_sep

\{

/indexed_colorspace_dict AGMCORE_gget begin

begin

URL: http://mc.manuscriptcentral.com/tandf/tmph 
1

2

3

4

5

6

7

8

9

10

11

12

13

14

15

16

17

18

19

20

21

22

23

24

25

26

27

28

29

30

31

32

33

34

35

36

37

38

39

40

41

42

43

44

45

46

47

48

49

50

51

52

53

54

55

56

57

58

59

60

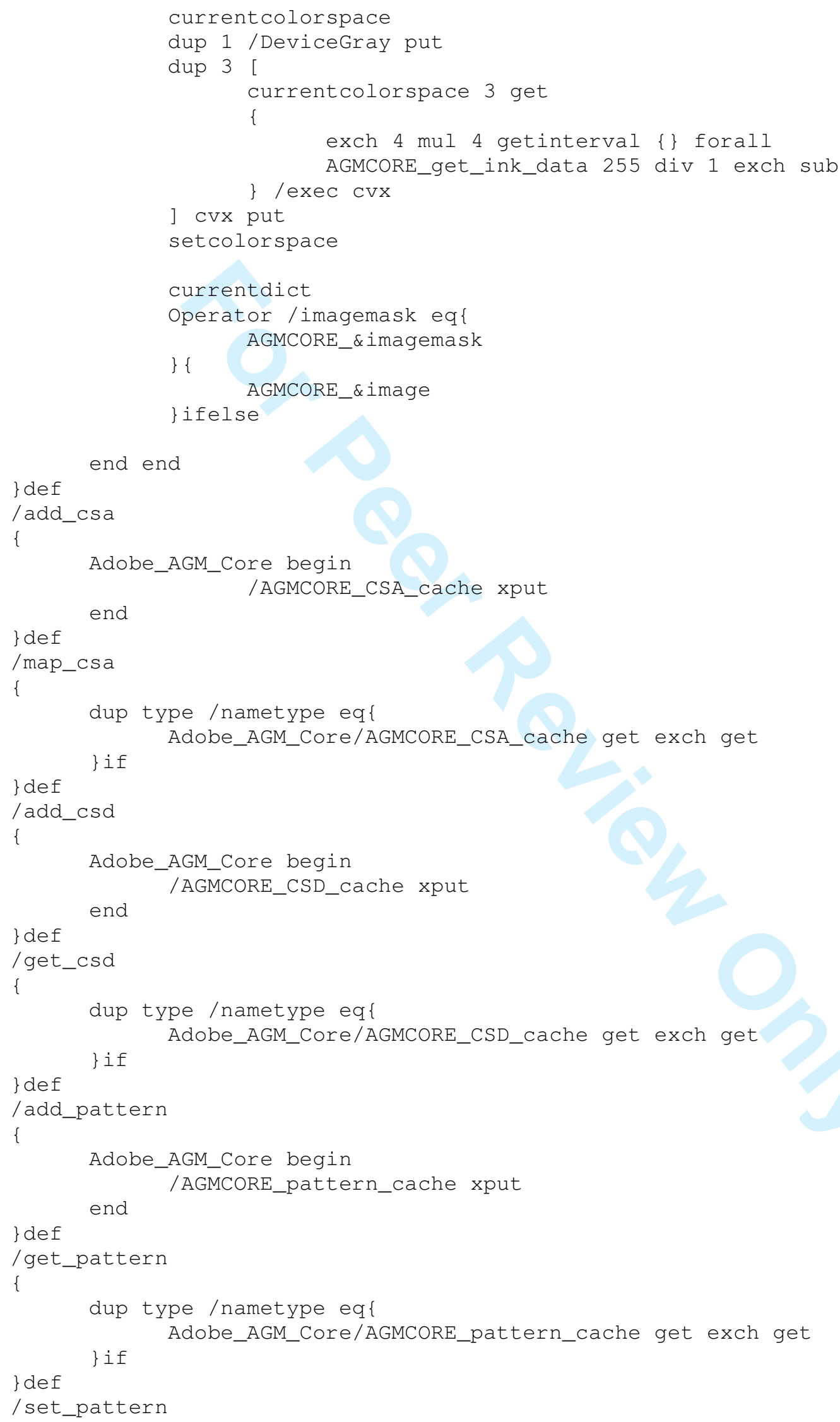

URL: http://mc.manuscriptcentral.com/tandf/tmph 
\{

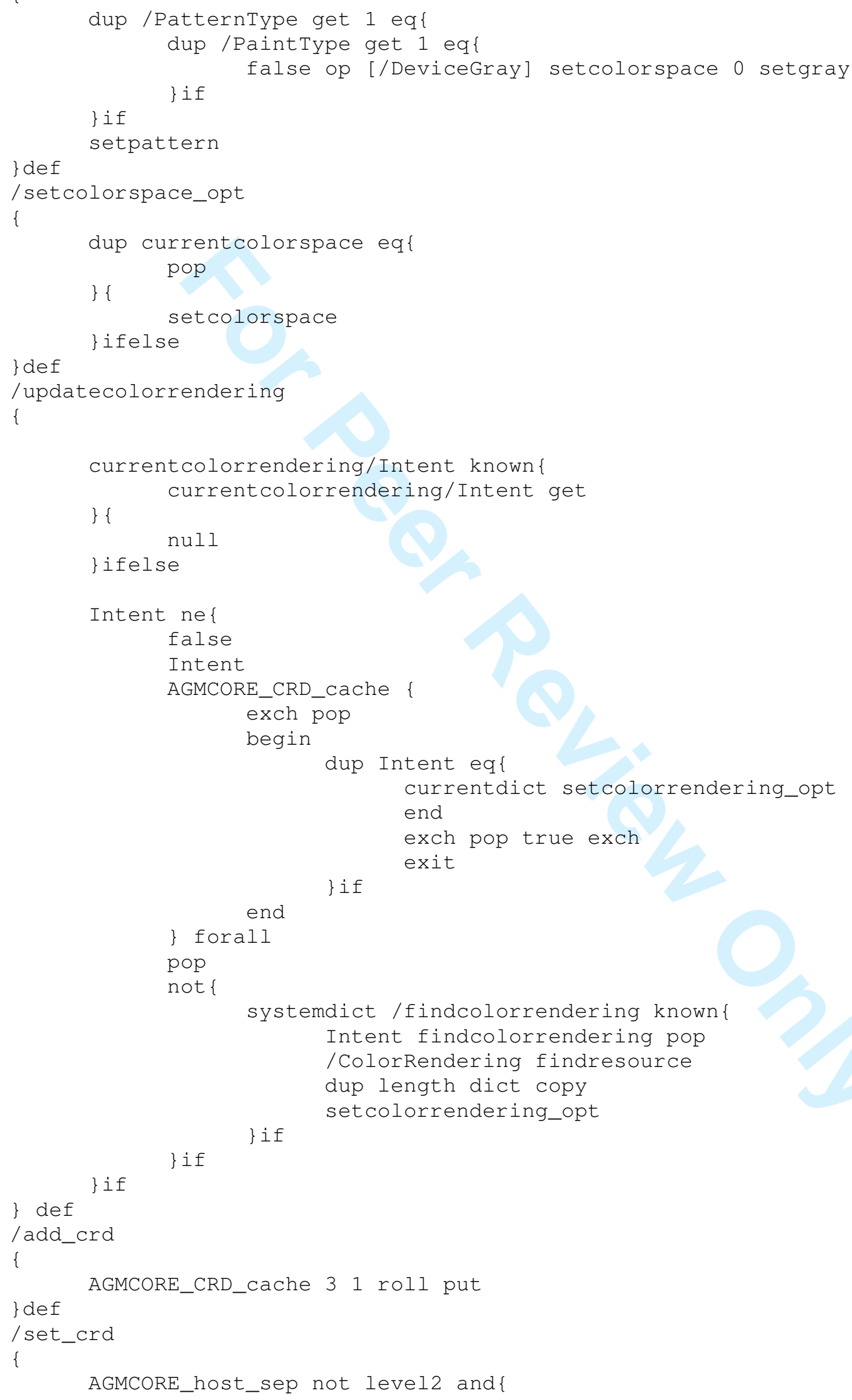

URL: http://mc.manuscriptcentral.com/tandf/tmph 


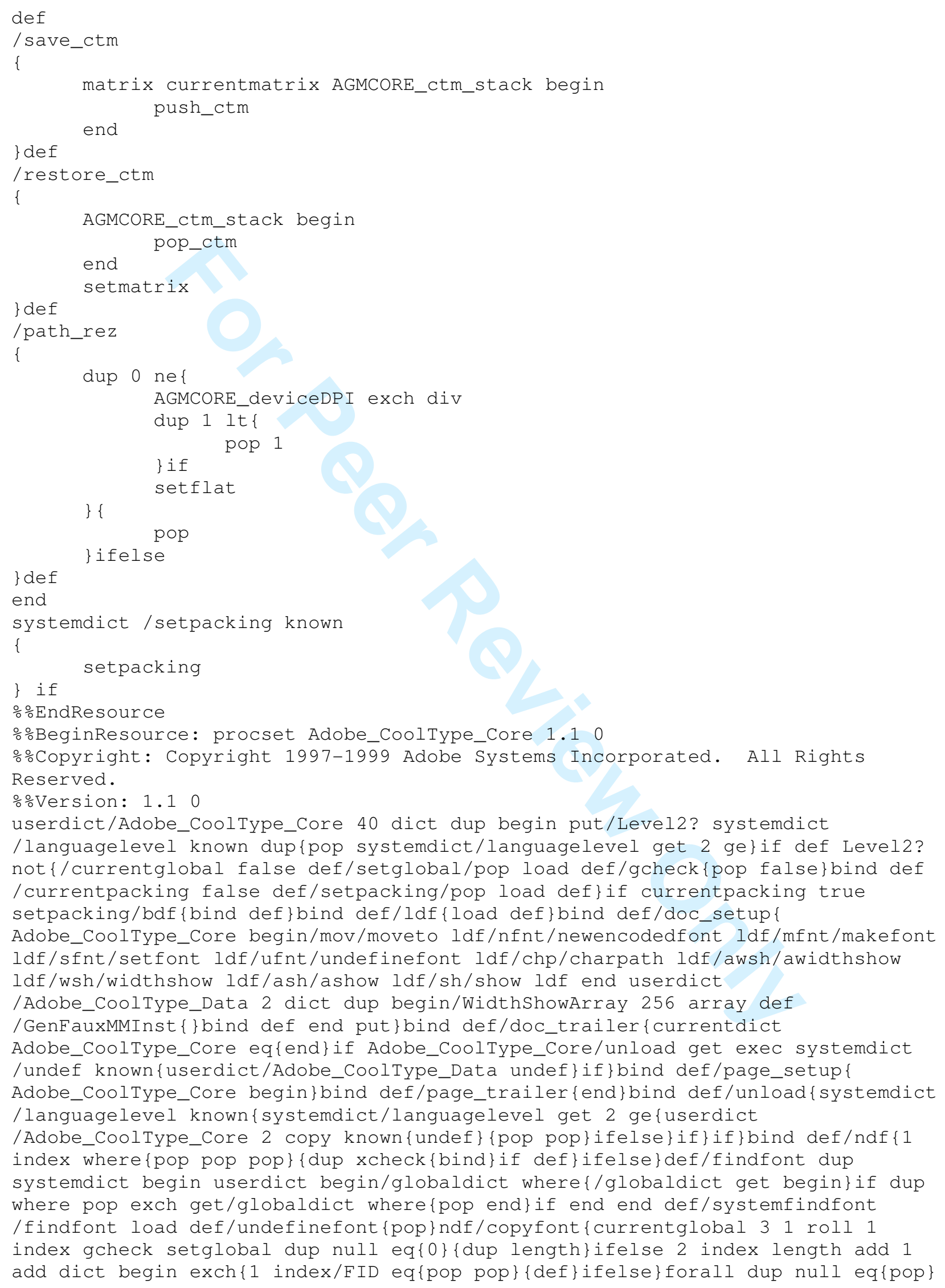


$\{\{$ def $\}$ forall\}ifelse currentdict end exch setglobal\}bdf/newencodedfont ffindfont dup length dict begin $\{1$ index/FID ne\{def\}\{pop pop\}ifelse\}forall/Encoding exch def currentdict end definefont pop\}bdf/scff\{\$SubstituteFont begin dup type /stringtype eq\{dup length exch\}\{null\}ifelse/\$sname exch def/\$slen exch def /\$fontname 1 index dup type/stringtype eq\{cvn\}if def/\$used false def end findfont \$SubstituteFont begin \$usedfcurrentglobal exch dup gcheck setglobal null copyfont begin/FontName \$fontname def/currentdistillerparams where\{pop\} /FontInfo currentdict/FontInfo known\{FontInfo null copyfont\}\{2 dict\}ifelse dup begin/ItalicAngle \$italicangle def/FontMatrix FontMatrix[1 0 ItalicAngle dup sin exch cos div 10 0]matrix concatmatrix readonly end 42 roll def def ifelse FontName currentdict end definefont exch setglobal\}if/\$sname null def /\$slen 0 def end\}bdf/ConcatStrings \{dup 0 exch\{length add\}forall string exch 0 exch\{3 copy putinterval length add\}forall pop\}bind def/BeginFauxMMFont $\{1$ index findfont pop 2 array astore ConcatStrings 31 roll(\$)exch 3 array astore ConcatStrings exch findfont currentglobal exch dup gcheck setglobal null copyfont/tempfontname exch definefont pop exch 12 dict begin/FontName exch def /FontType 0 def/FMapType 6 def/FontMatrix matrix def/PaintType 0 def\}bind def /EndFauxMMFont \{currentdict end dup/FontName get exch definefont exch setglobal exch mfnt sfnt\}bind def/GenFauxMMInst \{Adobe_CoolType_Data/GenFauxMMInst get exec\}bind def/DefFauxMMInst \{Adobe_CoolType_Data exch/GenFauxMMInst exch bind put\}bind def/WSALoop 4 copy 1 index add 1 sub 1 exch $\{3$ copy put pop 1 add for pop pop 32 roll add exch 256 add\}bind def/BuildWidthShowArray\{currentfont begin FontType 0 eq dup\{pop FMapType 6 eq\}if\{SubsVector dup length 1 sub 1 exch getinterval\}\{() \} ifelse end Adobe_CoolType_Data/WidthShowArray get 0043 roll\{WSALoop\}forall 2562 index sub WSALoop pop pop pop\}bind def/wsa\{ Adobe_CoolType_Data/WidthShowArray get\}bind def/?set\{3 index findfont dup /FontName known\{dup/FontName get/Courier eq\{4 index/Courier eq\}\{true\}ifelse exch/DistillerFauxFont known not and \{pop pop false\}ifelse $\{$ exch pop exch pop exch 2 dict dup/Found 3 index put exch findfont exch\}\{exch exec exch findfont 2 dict dup/Downloaded 65 roll put\}ifelse dup/FontName 4 index put copyfont definefont pop\}bind def/?str1 256 string def/?str2 256 string def/?add\{1 index type/integertype eq\{exch true 42 \} ffalse 31 \} ifelse roll 1 index findfont /Downloaded known \{exec exch\{exch ?str2 cvs exch findfont/Downloaded get 1 dict begin/Downloaded 1 index def ?str 1 cvs length ?str 11 index 1 add 3 index putinterval exch length 1 add 1 index add ?str 12 index(*)putinterval ?str 10 2 index getinterval cvn findfont ?str1 3 index(l)putinterval 2 dict dup /FontName ?str1 06 index getinterval cvn put dup/Downloaded Downloaded put end copyfont dup/FontName get exch definefont pop pop pop\}\{pop\}ifelse\} pop exch\{findfont dup/Found get dup length exch ?str1 cvs pop ?str1 1 index() putinterval ?str1 1 index 1 add 4 index ?str2 cvs putinterval ?str1 exch 0 exch 54 roll ?str2 cvs length 1 add add getinterval cvn 1 dict exch 1 index exch/FontName exch put copyfont dup/FontName get exch definefont pop\}\{pop\} ifelse\}ifelse\}bind def/?sh\{currentfont/Downloaded known exch\} if pop\}bind def /?chp\{currentfont/Downloaded known\{pop\}\{false chp\}ifelse\}bind def/?mv\{ currentfont/Downloaded known \{moveto pop pop\} \{pop pop moveto\}ifelse\}bind def setpacking end/currentdistillerparams where dup\{pop pop currentdistillerparams /CannotEmbedFontPolicy 2 copy known\{get/Error eq\}\{pop pop false\}ifelse\}if not 1 dict begin/SubstituteFont dup \$error exch 2 copy known\{get\} \{pop pop\{pop /Courier\}bind\}ifelse def userdict begin/\$SubstituteFont 20 dict dup begin / \$used false def end def end\{countdictstack array dictstack 0 get begin userdict begin \$SubstituteFont begin/\$str 128 string def/\$fontpat 128 string def/\$slen 0 def/\$sname null def/\$match false def/\$fontname null def/\$used false def/\$fontname null def/\$italicangle 26.5 def/\$findfontByEnum $\{$ dup /\$fontname exch def \$sname null eq\{\$str cvs dup length \$slen sub \$slen getinterval\}\{pop \$sname\} ifelse \$fontpat dup 0 (fonts/*)putinterval exch 7 exch putinterval/\$match false def mark\{\$fontpat 0 \$slen 7 add getinterval\{/\$match exch def exit\}\$str filenameforall\}stopped cleartomark/\$slen 0 def \$match false 
ne $\{\$$ match (fonts/) anchorsearch pop pop cvn/\$used true def\}\{/Courier\}ifelse\}bind def/\$ROS 1 dict dup begin/Adobe 4 dict dup begin/Japan1[/Ryumin-Light /HeiseiMin-W3/GothicBBB-Medium/HeiseiKakuGo-W5/HeiseiMaruGo-W4/Jun101-Light ] def/Koreal [/HYSMyeongJo-Medium/HYGoThic-Medium] def/GB1 [/STSong-Light /STHeiti-Regular]def/CNS1[/MKai-Medium/MHei-Medium]def end def end def /\$cmapname null def/\$buildfontname\{length \$str 1 index(-)putinterval 1 add \$str 1 index \$cmapname \$fontpat cvs putinterval \$cmapname length add \$str exch 0 exch getinterval cvn/\$used true def\}bind def/\$findfontByROS\{/\$fontname exch def $\$ R O S$ Registry 2 copy known\{get Ordering 2 copy known\{get\}\{pop pop[]\}ifelse \} \{pop pop[]\}ifelse false exch\{dup/CIDFont resourcestatus\{pop pop save 1 index /CIDFont findresource dup/WidthsOnly known\{dup/Widthsonly get\}\{false\}ifelse exch pop exch restore\{pop\} \{exch pop true exit\}ifelse\}\{pop\}ifelse\}forall\{\$str cvs \$buildfontname\} ffalse (*) \{save exch dup/CIDFont findresource dup/WidthsOnly known $\{$ dup/Widthsonly get not\}\{true\}ifelse exch/CIDSystemInfo get dup/Registry get Registry eq exch/Ordering get Ordering eq and and fexch restore exch pop true exit\} \{pop restore\}ifelse\}\$str/CIDFont resourceforall\{\$buildfontname\} \{ \$fontname \$findfontByEnum\}ifelse\}ifelse\}bind def end end currentdict/\$error known currentdict/languagelevel known and dup\{pop \$error/SubstituteFont known if dup\{\$error\}\{Adobe_CoolType_Core\}ifelse begin\{/SubstituteFont/CMap/Category resourcestatus\{pop pop $\{$ SubstituteFont begin dup length $\$$ slen gt \$sname null ne or \$slen 0 gt and\{\$sname null eq\{dup \$str cvs dup length \$slen sub \$slen getinterval cvn\}\{\$sname\}ifelse dup/CMap resourcestatus\{pop pop dup/\$cmapname exch def/CMap findresource/CIDSystemInfo get\{def\}forall \$findfontByROS\}\{pop \$findfontByEnum\}ifelse\} \{//SubstituteFont exec\}ifelse/\$slen 0 def end\}\}\{\{ \$SubstituteFont begin dup length \$slen gt \$sname null ne or \$slen 0 gt and \$findfontByEnum\}\{//SubstituteFont exec\}ifelse end\}\}ifelse bind readonly def\} \{ /findfont\{\$SubstituteFont begin dup systemfindfont \$slen 0 gt \$sname null ne 3 index length \$slen gt or and\{dup/FontName get dup/Courier eq exch 3 index ne and \{pop \$findfontByEnum findfont dup maxlength 1 add dict begin\{1 index/FID eq \{pop pop\}\{def\}ifelse\}forall \$fontname currentdict end definefont\} \{exch pop\} ifelse\}\{exch pop\}ifelse end\}bind def\}ifelse end end\}if end

으을

$\because \%$ EndProlog

$\therefore \%$ Beginsetup

Adobe_AGM_Core/AGMCORE_err_strings 3 dict dup begin /AGMCORE_bad_environ (Environment not satisfactory for this job, which requests at least PostScript Level $\wedge$ and at least PostScript version ^. Ensure that the PPD is correct or that the PostScript level requested is supported by this printer. ) def /AGMCORE_color_space_onhost_seps (This job contains colors that will not separate with on-host methods. ) def /AGMCORE_invalid_color_space (This job contains an invalid color space. ) def end put

22010 true Adobe_AGM_Core/doc_setup get exec Adobe_CoolType_Core/doc_setup get exec

$\therefore$ EndSetup

을

$\therefore \%$ EndPageComments 응 BeginPageSetup

Adobe_AGM_Core/page_setup get exec Adobe_CoolType_Core/page_setup get exec 을 EndPagesetup Adobe_AGM_Core/AGMCORE_save save ddf mark 10 


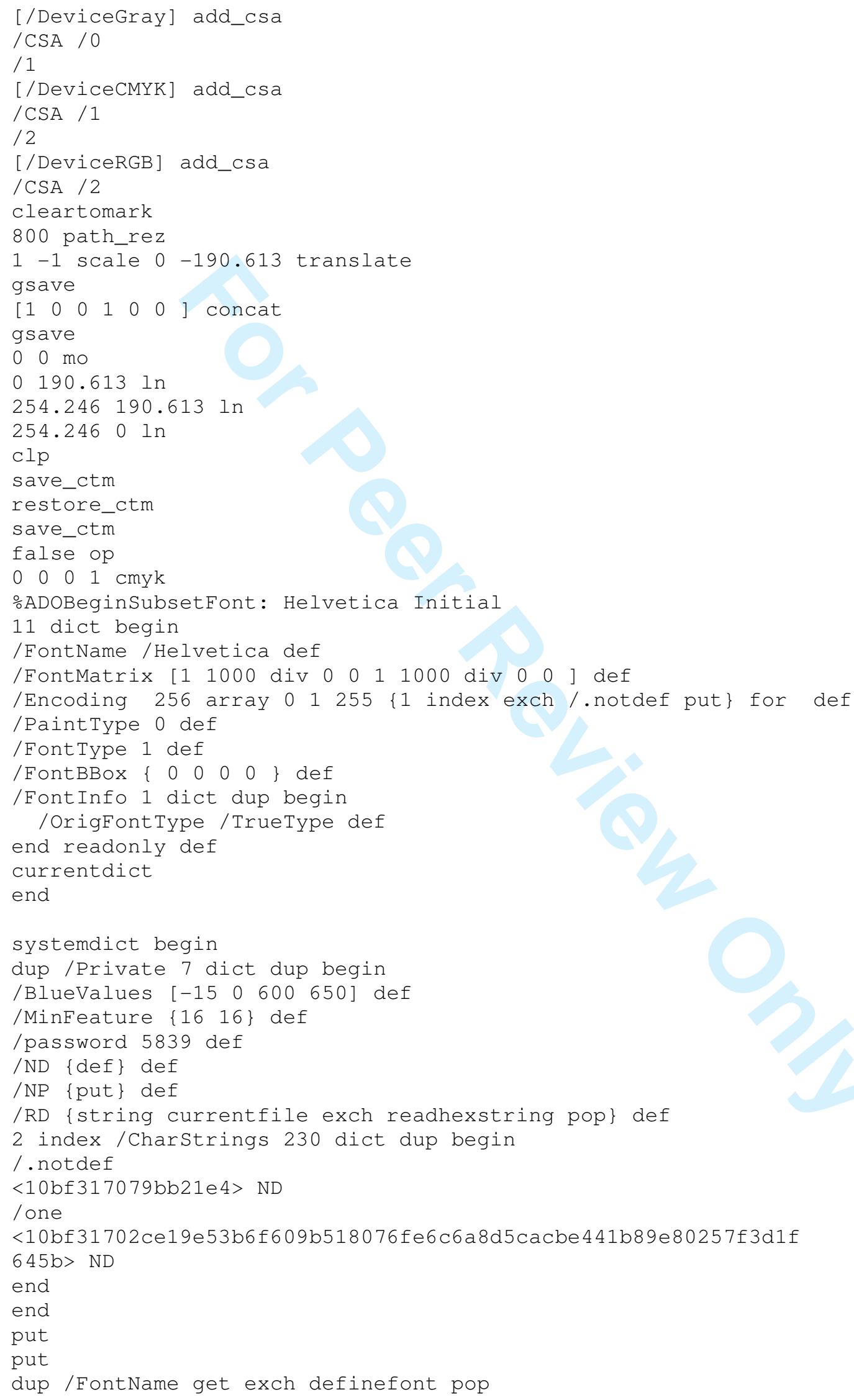




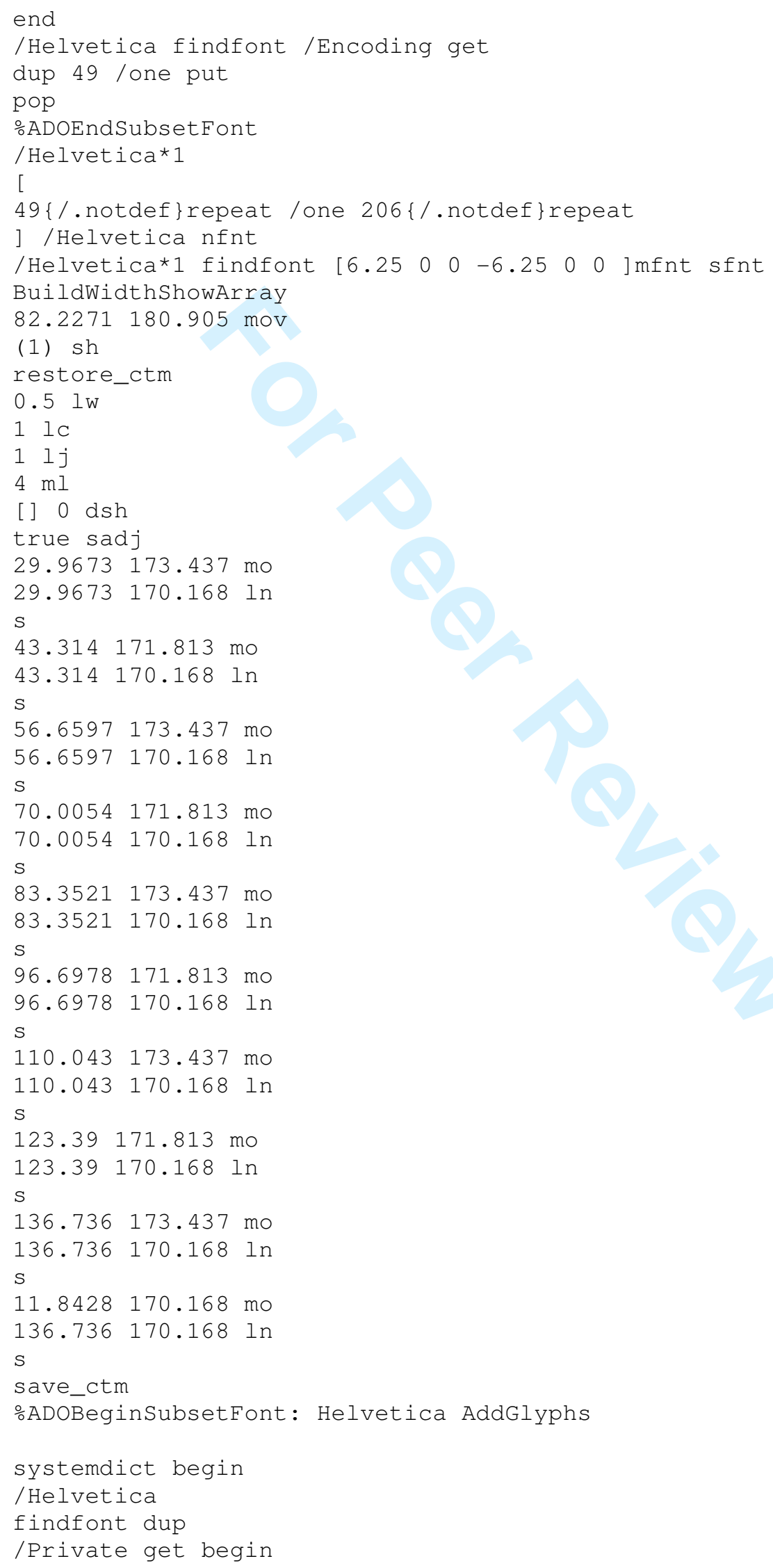




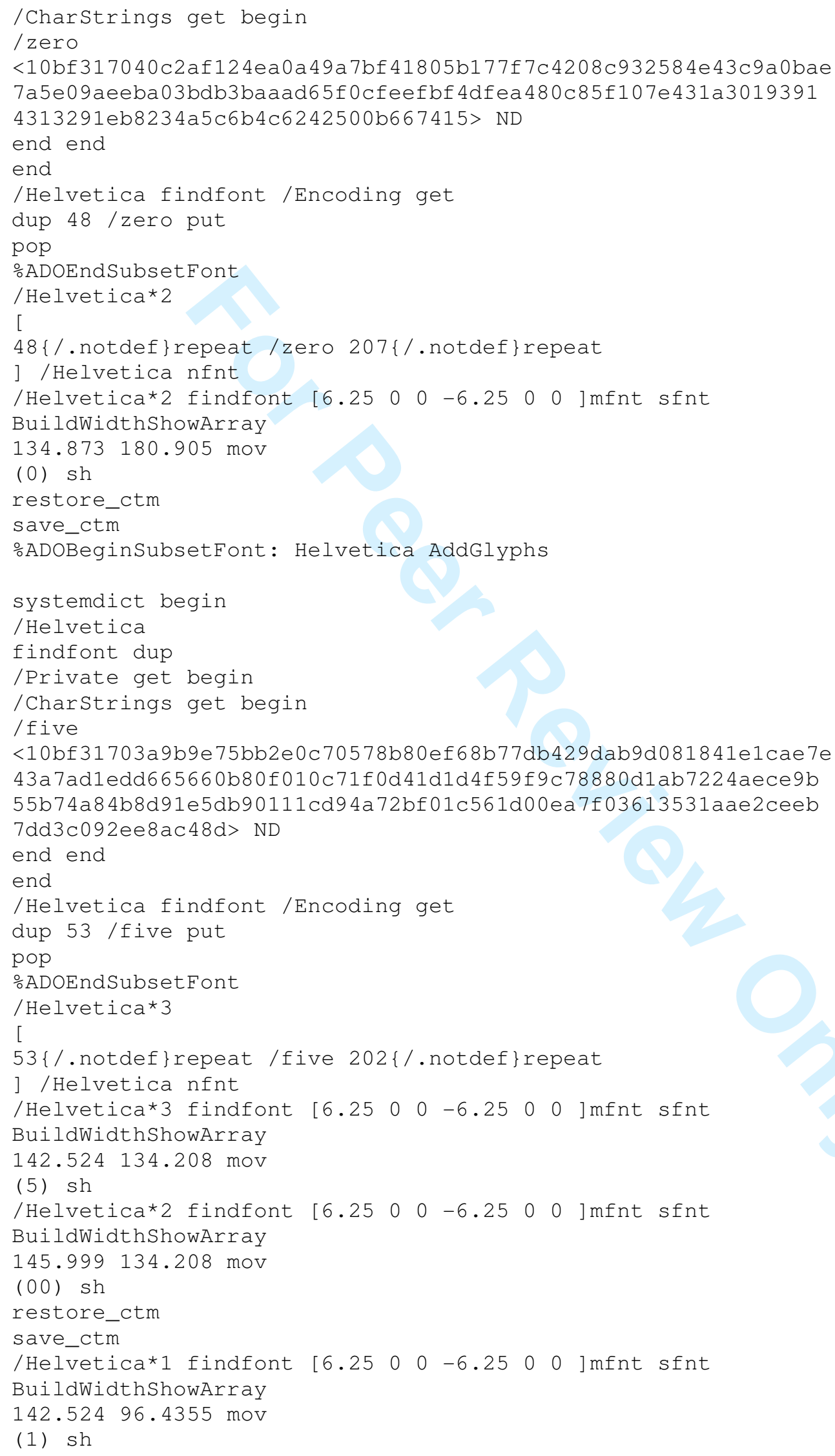


/Helvetica*2 findfont $\left[\begin{array}{lllllll}6.25 & 0 & 0 & -6.25 & 0 & 0\end{array}\right] \mathrm{mfnt}$ sfnt BuildWidthShowArray

$145.99996 .4355 \mathrm{mov}$ (000) sh

restore_ctm

$140.37170 .168 \mathrm{mo}$ $137.475170 .168 \mathrm{ln}$

$\mathrm{s}$

$136.98151 .293 \mathrm{mo}$ $136.98151 .293 \mathrm{ln}$

$\mathrm{s}$

$140.253 \quad 132.418 \mathrm{mo}$ 136.98132 .418 ln

$\mathrm{s}$

$136.98113 .521 \mathrm{mo}$ $136.98 \quad 113.521 \mathrm{ln}$

$\mathrm{s}$

$140.25394 .6465 \mathrm{mo}$

$136.9894 .6465 \mathrm{ln}$

$\mathrm{s}$

$136.98 \quad 75.7715 \mathrm{mo}$

$136.9875 .7715 \mathrm{ln}$

$\mathrm{s}$

$140.25356 .897 \mathrm{mo}$

$136.98 \quad 56.897 \mathrm{ln}$

$\mathrm{s}$

$136.98 \quad 37.9985 \mathrm{mo}$

$136.98 \quad 37.9985 \mathrm{ln}$

$\mathrm{s}$

$\begin{array}{lll}136.98 & 0.25 \mathrm{mo}\end{array}$

$136.98 \quad 0.25 \quad$ ln

$\mathrm{s}$

$136.98 \quad 170.168 \mathrm{mo}$

$136.9816 .583 \mathrm{ln}$

$\mathrm{s}$

$1 \mathrm{lw}$

$136.736170 .096 \mathrm{mo}$

$136.722169 .952 \mathrm{ln}$

$136.694169 .785 \mathrm{ln}$

$136.653169 .643 \mathrm{ln}$

$136.583169 .499 \mathrm{ln}$

$136.512169 .332 \mathrm{ln}$

$136.429169 .188 \mathrm{ln}$

$136.332169 .046 \mathrm{ln}$

$136.219168 .879 \mathrm{ln}$

$136.094168 .735 \mathrm{ln}$

$135.955168 .593 \mathrm{ln}$

$135.816168 .426 \mathrm{ln}$

$135.648168 .282 \mathrm{ln}$

$135.466168 .139 \mathrm{ln}$

$135.286167 .972 \mathrm{ln}$

$135.09167 .829 \mathrm{ln}$

$134.867167 .686 \mathrm{ln}$

$134.658167 .519 \mathrm{ln}$

$134.42167 .376 \mathrm{ln}$

$134.169167 .232 \mathrm{ln}$

$133.919167 .065 \mathrm{ln}$

URL: http://mc.manuscriptcentral.com/tandf/tmph 


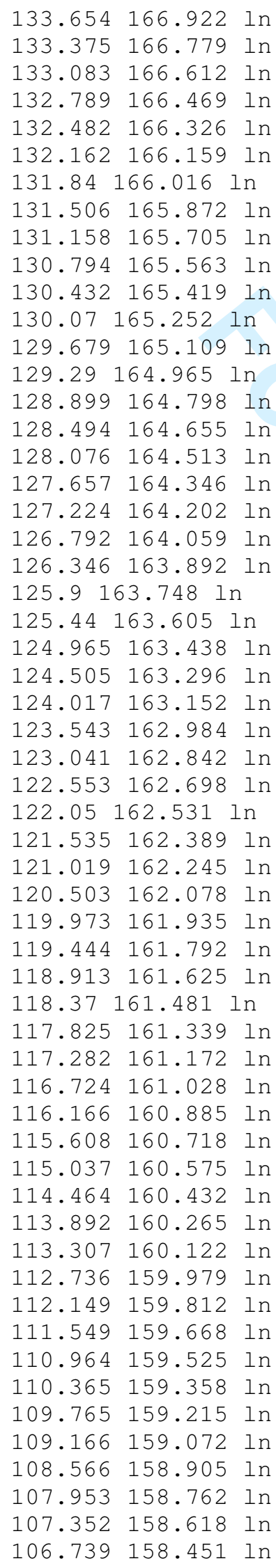


$106.125158 .309 \mathrm{ln}$ $105.511158 .165 \mathrm{ln}$ 104.883157 .998 In $104.27157 .855 \mathrm{ln}$ $103.642157 .711 \mathrm{ln}$ $103.029157 .544 \mathrm{ln}$ $102.402157 .401 \mathrm{ln}$ $101.774 \quad 157.259$ In $101.146157 .092 \mathrm{ln}$ $100.519156 .948 \mathrm{ln}$ $99.8774156 .805 \mathrm{ln}$ $99.2495156 .638 \mathrm{ln}$ $98.6226 \quad 156.494 \mathrm{ln}$ $97.981156 .352 \mathrm{ln}$ $97.353156 .185 \mathrm{ln}$ $96.7114156 .042 \mathrm{ln}$ $96.0835155 .898 \mathrm{ln}$ $95.4429155 .755 \mathrm{ln}$ 94.8003155 .588 In 94.1733155 .445 In $93.5317155 .302 \mathrm{ln}$ $92.8901155 .135 \mathrm{ln}$ $92.2485154 .991 \mathrm{ln}$ 91.6216154 .848 ln $90.98154 .681 \mathrm{ln}$ $90.3384154 .538 \mathrm{ln}$ $89.6968154 .396 \mathrm{ln}$ $89.0698154 .228 \mathrm{ln}$ $88.4282154 .085 \mathrm{ln}$ $87.7866153 .941 \mathrm{ln}$ $87.1587 \quad 153.774 \quad \mathrm{ln}$ $86.5171153 .631 \mathrm{ln}$ $85.8901153 .488 \mathrm{ln}$ $85.2485153 .321 \mathrm{ln}$ $84.6206153 .179 \mathrm{ln}$ $83.979153 .034 \mathrm{ln}$ $83.3521152 .868 \mathrm{ln}$ $82.7241 \quad 152.725 \mathrm{ln}$ $82.0972152 .581 \mathrm{ln}$ $81.4692152 .414 \mathrm{ln}$ $80.8413152 .271 \mathrm{ln}$ 80.2144152 .128 In $79.5864151 .961 \mathrm{ln}$ $78.9731151 .817 \mathrm{ln}$ $78.3452151 .675 \mathrm{ln}$ $77.7319151 .508 \mathrm{ln}$ $77.1177151 .364 \mathrm{ln}$ 76.5044151 .222 In $75.8901151 .055 \mathrm{ln}$ $75.2778150 .911 \mathrm{ln}$ $74.6636150 .768 \mathrm{ln}$ $74.0493150 .601 \mathrm{ln}$ $73.4497150 .458 \mathrm{ln}$ $72.8501150 .314 \mathrm{ln}$ $72.2368150 .147 \mathrm{ln}$ $71.6372150 .005 \mathrm{ln}$ $71.0522149 .861 \mathrm{ln}$ 
$70.4517 \quad 149.694 \mathrm{ln}$ $69.8657149 .551 \mathrm{ln}$ 69.2661149 .408 In $68.6812149 .241 \mathrm{ln}$ 68.0952149 .098 ln $67.5093148 .955 \mathrm{ln}$ $66.937148 .788 \mathrm{ln}$ $66.3521148 .645 \mathrm{ln}$ $65.7798148 .501 \mathrm{ln}$ $65.2085148 .334 \mathrm{ln}$ $64.6362148 .191 \mathrm{ln}$ $64.0786148 .048 \mathrm{ln}$ $63.52147 .881 \mathrm{ln}$ 62.9487147 .738 ln 62.3911147 .594 In $61.8472147 .427 \mathrm{ln}$ $61.2896147 .284 \mathrm{ln}$ $60.7456147 .142 \mathrm{ln}$ $60.2017146 .975 \mathrm{ln}$ $59.6577 \quad 146.831 \mathrm{ln}$ 59.1138146 .688 ln $58.5835146 .521 \mathrm{ln}$ $58.0542146 .377 \mathrm{ln}$ $57.5239146 .234 \mathrm{ln}$ $56.9946146 .067 \mathrm{ln}$ $56.478145 .925 \mathrm{ln}$ $55.9614145 .78 \mathrm{ln}$ $55.4458145 .613 \mathrm{ln}$ $54.9302145 .471 \mathrm{ln}$ $54.4282145 .327 \mathrm{ln}$ $53.9116 \quad 145.16 \mathrm{ln}$ $53.4106145 .018 \mathrm{ln}$ $52.9224 \quad 144.874 \quad \mathrm{ln}$ $52.4194144 .707 \mathrm{ln}$ $51.9321144 .563 \mathrm{ln}$ $51.4438144 .421 \mathrm{ln}$ $50.9692144 .254 \mathrm{ln}$ $50.4819144 .11 \mathrm{ln}$ $50.0073143 .968 \mathrm{ln}$ $49.5327143 .801 \mathrm{ln}$ $49.0728 \quad 143.657 \mathrm{ln}$ $48.6128143 .514 \mathrm{ln}$ $48.1528143 .347 \mathrm{ln}$ $47.6929143 .204 \mathrm{ln}$ $47.2319143 .061 \mathrm{ln}$ $46.7866 \quad 142.894 \quad$ In 46.3394142 .751 In $45.9067142 .607 \mathrm{ln}$ $45.4614142 .44 \mathrm{ln}$ $45.0288142 .297 \mathrm{ln}$ $44.5972142 .154 \mathrm{ln}$ $44.1782141 .987 \mathrm{ln}$ $43.7603141 .844 \mathrm{ln}$ $43.3413141 .701 \mathrm{ln}$ $42.9233141 .558 \mathrm{ln}$ $42.519141 .391 \mathrm{ln}$ 42.1147141 .247 In 
$41.7095141 .104 \mathrm{ln}$ $41.3198140 .938 \mathrm{ln}$ $40.9282140 .794 \mathrm{ln}$ $40.5376140 .651 \mathrm{ln}$ $40.1479140 .484 \mathrm{ln}$ $39.771 \quad 140.34 \mathrm{ln}$ $39.395140 .197 \mathrm{ln}$ $39.0181140 .03 \mathrm{ln}$ $38.6558139 .888 \mathrm{ln}$ $38.2935139 .744 \mathrm{ln}$ $37.9312139 .577 \mathrm{ln}$ $37.5679139 .434 \mathrm{ln}$ $37.2192139 .29 \mathrm{ln}$ $36.8706 \quad 139.123 \mathrm{ln}$ $36.5356138 .98 \mathrm{ln}$ $36.187 \quad 138.838 \mathrm{ln}$ $35.853 \quad 138.671 \mathrm{ln}$ $35.5317138 .526 \mathrm{ln}$ $35.1978138 .384 \mathrm{ln}$ $34.8765138 .217 \mathrm{ln}$ $34.5562138 .073 \mathrm{ln}$ $34.2485137 .931 \mathrm{ln}$ $33.9419137 .764 \mathrm{ln}$ $33.6353137 .62 \mathrm{ln}$ $33.3286 \quad 137.477 \mathrm{ln}$ $33.0356 \quad 137.31 \mathrm{ln}$ $32.7427137 .167 \mathrm{ln}$ $32.4497137 .023 \mathrm{ln}$ $32.1704136 .856 \mathrm{ln}$ $31.8921136 .714 \mathrm{ln}$ $31.6128136 .57 \mathrm{ln}$ $31.3345136 .403 \mathrm{ln}$ $31.0693136 .26 \mathrm{ln}$ $30.8042136 .117 \mathrm{ln}$ $30.5532135 .95 \mathrm{ln}$ $30.2881135 .807 \mathrm{ln}$ $30.0371135 .663 \mathrm{ln}$ $29.8003135 .497 \mathrm{ln}$ $29.5493 \quad 135.354 \mathrm{ln}$ $29.312135 .21 \mathrm{ln}$ $29.0752135 .043 \mathrm{ln}$ $28.8521134 .9 \mathrm{ln}$ $28.6289134 .757 \mathrm{ln}$ $28.4058134 .59 \mathrm{ln}$ $28.1826 \quad 134.447 \mathrm{ln}$ $27.9731134 .304 \mathrm{ln}$ $27.7642134 .137 \mathrm{ln}$ $27.5547 \quad 133.993 \mathrm{ln}$ $27.3599133 .851 \mathrm{ln}$ $27.1646133 .684 \mathrm{ln}$ $26.9692133 .54 \mathrm{ln}$ $26.7739133 .396 \mathrm{ln}$ $26.5928133 .23 \mathrm{ln}$ $26.4116133 .086 \mathrm{ln}$ $26.23132 .943 \mathrm{ln}$ $26.0625132 .776 \mathrm{ln}$ $25.8955132 .634 \mathrm{ln}$ 


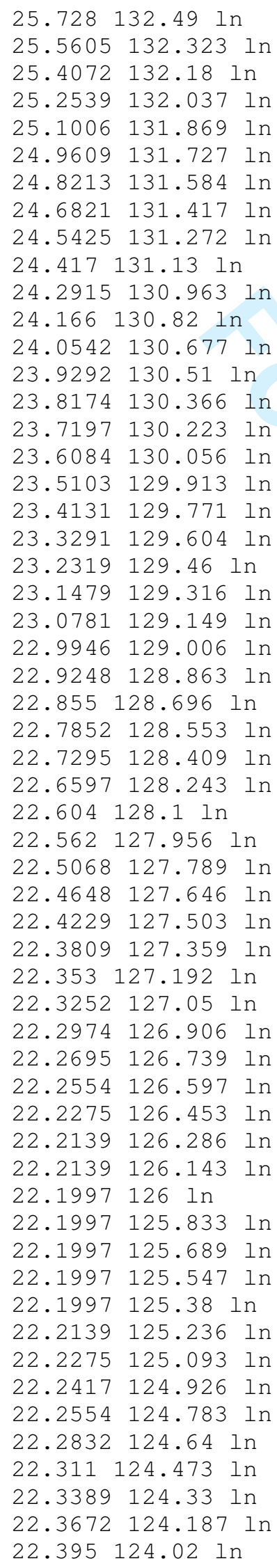


$22.437 \quad 123.876 \quad$ ln $22.4785123 .733 \mathrm{ln}$ $22.5205123 .566 \mathrm{ln}$ $22.562123 .423 \mathrm{ln}$ $22.6182123 .28 \mathrm{ln}$ $22.6597123 .112 \mathrm{ln}$ $22.7158122 .969 \mathrm{ln}$ $22.7715122 .826 \mathrm{ln}$ $22.8413122 .659 \mathrm{ln}$ $22.897122 .517 \mathrm{ln}$ $22.9668122 .373 \mathrm{ln}$ $23.0361122 .206 \mathrm{ln}$ $23.106122 .063 \mathrm{ln}$ 23.1758121 .919 ln 23.2598121 .752 ln $23.3433121 .609 \mathrm{ln}$ $23.4268121 .467 \mathrm{ln}$ $23.5103121 .3 \mathrm{ln}$ $23.5942121 .155 \mathrm{ln}$ $23.6919121 .013 \mathrm{ln}$ $23.7754120 .846 \mathrm{ln}$ $23.873120 .702 \mathrm{ln}$ $23.9707120 .56 \mathrm{ln}$ $24.0825120 .393 \mathrm{ln}$ $24.1802120 .249 \mathrm{ln}$ $24.2915120 .105 \mathrm{ln}$ $24.3892119 .938 \mathrm{ln}$ $24.5005119 .796 \mathrm{ln}$ $24.6265119 .652 \mathrm{ln}$ $24.7378119 .485 \mathrm{ln}$ $24.8491119 .343 \mathrm{ln}$ $24.9746119 .199 \mathrm{ln}$ $25.1006119 .032 \mathrm{ln}$ $25.2261118 .889 \mathrm{ln}$ $25.3516118 .746 \mathrm{ln}$ $25.4771118 .579 \mathrm{ln}$ $25.6162118 .436 \mathrm{ln}$ $25.7559118 .293 \mathrm{ln}$ $25.8813118 .126 \mathrm{ln}$ $26.0205117 .982 \mathrm{ln}$ $26.1743117 .839 \mathrm{ln}$ $26.3135117 .672 \mathrm{ln}$ $26.4531117 .529 \mathrm{ln}$ $26.6064117 .386 \mathrm{ln}$ $26.7598117 .219 \mathrm{ln}$ $26.9136117 .076 \mathrm{ln}$ $27.0669116 .933 \mathrm{ln}$ $27.2202116 .766 \mathrm{ln}$ $27.3735116 .622 \mathrm{ln}$ $27.541116 .479 \mathrm{ln}$ $27.6943116 .313 \mathrm{ln}$ $27.8618116 .169 \mathrm{ln}$ $28.0288116 .026 \mathrm{ln}$ $28.1963115 .858 \mathrm{ln}$ $28.3638115 .715 \mathrm{ln}$ $28.5449115 .572 \mathrm{ln}$ $28.7124115 .405 \mathrm{ln}$ 
$28.8936115 .263 \mathrm{ln}$ $29.0752115 .119 \mathrm{ln}$ $29.2563114 .952 \mathrm{ln}$ $29.4375114 .809 \mathrm{ln}$ $29.6191114 .666 \mathrm{ln}$ $29.8003114 .498 \mathrm{ln}$ $29.9814114 .355 \mathrm{ln}$ $30.1768114 .213 \mathrm{ln}$ $30.3579114 .046 \mathrm{ln}$ $30.5532113 .901 \mathrm{ln}$ 30.7485113 .759 In 30.9434113 .592 In $31.1387 \quad 113.448$ ln 31.3345113 .306 ln 31.5435113 .162 In 31.7388112 .995 In 31.9478112 .852 In $32.1431112 .709 \mathrm{ln}$ 32.3521112 .542 In $32.561112 .398 \mathrm{ln}$ $32.77 \quad 112.256 \mathrm{ln}$ $32.98112 .089 \mathrm{ln}$ $33.189111 .945 \mathrm{ln}$ $33.3979111 .802 \mathrm{ln}$ $33.6206111 .635 \mathrm{ln}$ $33.8296111 .492 \mathrm{ln}$ $34.0532111 .349 \mathrm{ln}$ $34.2622111 .182 \mathrm{ln}$ $34.4858111 .038 \mathrm{ln}$ $34.7085110 .896 \mathrm{ln}$ $34.9321110 .729 \mathrm{ln}$ $35.1558110 .585 \mathrm{ln}$ $35.3784110 .442 \mathrm{ln}$ $35.6021110 .275 \mathrm{ln}$ $35.8384110 .132 \mathrm{ln}$ $36.061109 .988 \mathrm{ln}$ $36.2993109 .822 \mathrm{ln}$ $36.522109 .679 \mathrm{ln}$ $36.7593109 .535 \mathrm{ln}$ $36.9956109 .368 \mathrm{ln}$ 37.2192109 .226 ln $37.4565109 .082 \mathrm{ln}$ $37.6938108 .915 \mathrm{ln}$ $37.9312108 .771 \mathrm{ln}$ $38.1675108 .629 \mathrm{ln}$ $38.4048108 .461 \mathrm{ln}$ $38.6558108 .318 \mathrm{ln}$ $38.8921108 .176 \mathrm{ln}$ 39.1304108 .009 In $39.3813107 .865 \mathrm{ln}$ $39.6177107 .722 \mathrm{ln}$ $39.8696 \quad 107.555 \mathrm{ln}$ $40.1196107 .412 \mathrm{ln}$ $40.3569107 .269 \mathrm{ln}$ $40.6079107 .102 \mathrm{ln}$ $40.8589106 .959 \mathrm{ln}$ 41.1108106 .814 In 
$41.3618 \quad 106.647 \mathrm{ln}$ $41.6118106 .505 \mathrm{ln}$ $41.8628106 .362 \mathrm{ln}$ $42.1147106 .195 \mathrm{ln}$ $42.3657106 .052 \mathrm{ln}$ $42.6157105 .908 \mathrm{ln}$ $42.8667105 .741 \mathrm{ln}$ $43.1323105 .598 \mathrm{ln}$ $43.3833105 .455 \mathrm{ln}$ 43.6479105 .288 ln $43.8989105 .146 \mathrm{ln}$ 44.1646105 .002 In $44.4155104 .834 \mathrm{ln}$ $44.6802104 .691 \mathrm{ln}$ 44.9312104 .548 In $45.1958104 .381 \mathrm{ln}$ $45.4614104 .238 \mathrm{ln}$ 45.7261104 .095 In 45.9771103 .928 In $46.2417103 .784 \mathrm{ln}$ $46.5073103 .642 \mathrm{ln}$ $46.772103 .475 \mathrm{ln}$ $47.0366 \quad 103.331 \mathrm{ln}$ $47.3022103 .188 \mathrm{ln}$ $47.5669103 .021 \mathrm{ln}$ 47.8315102 .878 ln $48.0972102 .734 \mathrm{ln}$ $48.3618102 .567 \mathrm{ln}$ $48.6274 \quad 102.425 \mathrm{ln}$ $48.9058 \quad 102.281 \mathrm{ln}$ 49.1704102 .114 In $49.436101 .972 \mathrm{ln}$ $49.7007101 .828 \mathrm{ln}$ $49.9653101 .661 \mathrm{ln}$ $50.2446101 .518 \mathrm{ln}$ $50.5093101 .375 \mathrm{ln}$ $50.7739101 .208 \mathrm{ln}$ $51.0532101 .064 \mathrm{ln}$ $51.3188100 .922 \mathrm{ln}$ $51.5972100 .755 \mathrm{ln}$ $51.8618100 .611 \mathrm{ln}$ 52.1274100 .468 In $52.4067100 .301 \mathrm{ln}$ $52.6704100 .158 \mathrm{ln}$ $52.9497100 .015 \mathrm{ln}$ $53.215399 .8477 \mathrm{ln}$ 53.493799 .7051 ln $53.758399 .5615 \mathrm{ln}$ $54.037699 .3936 \mathrm{ln}$ $54.303299 .251 \mathrm{ln}$ $54.581599 .1084 \mathrm{ln}$ 54.860898 .9648 ln 55.125598 .7979 ln $55.403898 .6543 \mathrm{ln}$ $55.669498 .5107 \mathrm{ln}$ 55.948798 .3438 ln 56.213498 .2012 ln 


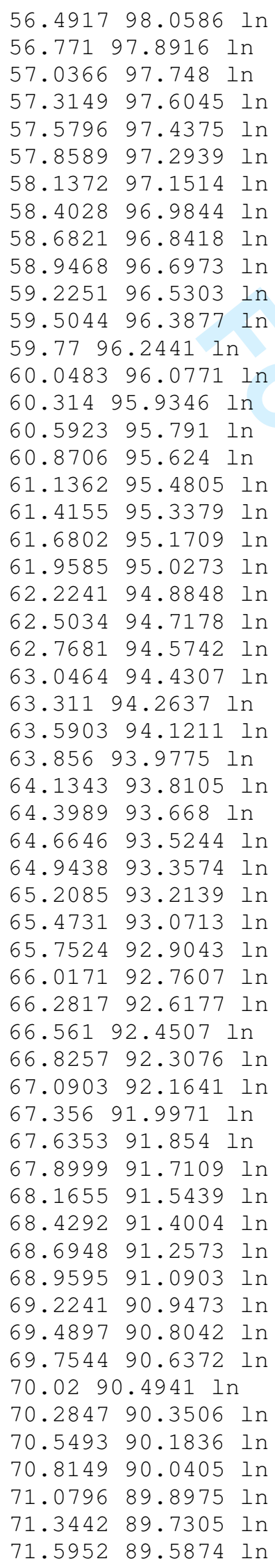


$71.8608 \quad 89.4438$ ln $72.124589 .2769 \mathrm{ln}$ 72.390189 .1338 ln $72.641188 .9907 \mathrm{ln}$ $72.906788 .8237 \mathrm{ln}$ $73.171488 .6807 \mathrm{ln}$ $73.422488 .5371 \mathrm{ln}$ $73.68788 .3701 \mathrm{ln}$ $73.93888 .2271 \mathrm{ln}$ $74.202688 .084 \mathrm{ln}$ $74.453687 .917 \mathrm{ln}$ $74.719287 .7739 \mathrm{ln}$ $74.970287 .6304 \mathrm{ln}$ $75.235887 .4634 \mathrm{ln}$ 75.486887 .3203 In $75.736887 .1772 \mathrm{ln}$ $75.987887 .0103 \mathrm{ln}$ $76.253486 .8672 \mathrm{ln}$ 76.504486 .7236 In 76.755486 .5566 ln $77.006386 .4136 \mathrm{ln}$ $77.257386 .2705 \mathrm{ln}$ $77.508386 .1035 \mathrm{ln}$ $77.759385 .9604 \mathrm{ln}$ $78.011285 .8169 \mathrm{ln}$ $78.261285 .6499 \mathrm{ln}$ $78.512285 .5068 \mathrm{ln}$ $78.763285 .3638 \mathrm{ln}$ $79.014285 .2207 \mathrm{ln}$ $79.265185 .0537 \mathrm{ln}$ 79.502484 .9102 In $79.753484 .7671 \mathrm{ln}$ $80.004484 .6001 \mathrm{ln}$ $80.241784 .457 \mathrm{ln}$ $80.492784 .314 \mathrm{ln}$ $80.72984 .147 \mathrm{ln}$ $80.98184 .0034 \mathrm{ln}$ $81.218383 .8604 \mathrm{ln}$ $81.469283 .6934 \mathrm{ln}$ $81.706583 .5503 \mathrm{ln}$ $81.942983 .4072 \mathrm{ln}$ $82.193883 .2402 \mathrm{ln}$ $82.431283 .0967 \mathrm{ln}$ $82.668582 .9536 \mathrm{ln}$ 82.905882 .7866 ln $83.142182 .6436 \mathrm{ln}$ $83.39482 .5005 \mathrm{ln}$ $83.631382 .3335 \mathrm{ln}$ $83.867782 .1899 \mathrm{ln}$ $84.10582 .0469 \mathrm{ln}$ 84.327681 .8799 ln $84.564981 .7368 \mathrm{ln}$ 84.802281 .5938 ln $85.039681 .4268 \mathrm{ln}$ $85.276981 .2832 \mathrm{ln}$ 85.498581 .1401 ln $85.7368 \quad 80.9731$ ln 


\begin{tabular}{|c|c|}
\hline 595 & \\
\hline 6.1958 & $80.687 \mathrm{ln}$ \\
\hline 6.433 & $80.52 \ln$ \\
\hline & 80.37651 \\
\hline & 80.2334 \\
\hline & 80.0664 \\
\hline & 331 \\
\hline & $3 \ln$ \\
\hline & 33 \\
\hline & 97 \\
\hline & 79.3 \\
\hline & \\
\hline & 79.0 \\
\hline & 78.8 \\
\hline & 78.70651 \\
\hline .36 & $78.563 \mathrm{ln}$ \\
\hline 50 & 78.41991 \\
\hline & 78.25 \\
\hline & 78.10 \\
\hline$\cdot 24$ & 77.96 \\
\hline & 77.79 \\
\hline & 377.65631 \\
\hline .896 & $77.5132 \mathrm{ln}$ \\
\hline 1.10 & $77.3462 \mathrm{ln}$ \\
\hline .32 & 577.20311 \\
\hline .53 & 577.06011 \\
\hline .74 & 576.8931 \\
\hline .97 & 76.74 \\
\hline .17 & 76.60 \\
\hline .38 & 76.4395 \\
\hline & 76.2 \\
\hline .82 & 376. \\
\hline & B 75. \\
\hline . & 75. \\
\hline & 75. \\
\hline .65 & $\begin{array}{lll}7 & 75.5327 & 1\end{array}$ \\
\hline 3.85 & $75.3896 \mathrm{ln}$ \\
\hline 4.06 & $75.2466 \mathrm{ln}$ \\
\hline 94.271 & $75.0796 \mathrm{ln}$ \\
\hline 4.487 & 74.936 ln \\
\hline 7.6899 & $74.793 \mathrm{ln}$ \\
\hline 7.88 & $374.626 \mathrm{ln}$ \\
\hline & 291 \\
\hline 28 & 74. \\
\hline & 74. \\
\hline & 74. \\
\hline 5.8 & 73. \\
\hline & 73. \\
\hline 5.25 & 73.5762 \\
\hline & 73. \\
\hline 6.69 & 73.26611 \\
\hline 96.89 & $73.123 \ln$ \\
\hline 97.08 & 72.97951 \\
\hline 97.2827 & $7 \quad 72.81251$ \\
\hline 97.478 & $72.6694 \mathrm{ln}$ \\
\hline & 372.5 \\
\hline 97.869 & 72.3594 \\
\hline
\end{tabular}


$107.61763 .9121 \mathrm{ln}$ $107.7763 .769 \mathrm{ln}$ $107.92463 .6021 \mathrm{ln}$ $108.09163 .459 \mathrm{ln}$ $108.23163 .3159 \mathrm{ln}$ $108.38463 .1489 \mathrm{ln}$ $108.53863 .0054 \mathrm{ln}$ $108.69162 .8623 \mathrm{ln}$ $108.84462 .6953 \mathrm{ln}$ $108.99862 .5522 \mathrm{ln}$ $109.13762 .4092 \mathrm{ln}$ $109.29162 .2417 \mathrm{ln}$ $109.44562 .0986 \mathrm{ln}$ $109.58361 .9556 \mathrm{ln}$ 109.73761 .7886 In $109.87661 .6455 \mathrm{ln}$ $110.0361 .5024 \mathrm{ln}$ $110.16961 .3354 \mathrm{ln}$ $110.30861 .1919 \mathrm{ln}$ $110.46161 .0488 \mathrm{ln}$ $110.60260 .8818 \mathrm{ln}$ $110.74160 .7388 \mathrm{ln}$ $110.88 \quad 60.5957 \mathrm{ln}$ $111.0260 .4287 \mathrm{ln}$ $111.16 \quad 60.2852 \mathrm{ln}$ $111.29960 .1421 \mathrm{ln}$ $111.43859 .9751 \mathrm{ln}$ $111.57859 .832 \mathrm{ln}$ $111.71759 .6885 \mathrm{ln}$ $111.85759 .5215 \mathrm{ln}$ $111.99659 .3784 \mathrm{ln}$ $112.13559 .2354 \mathrm{ln}$ $112.26159 .0684 \mathrm{ln}$ $112.458 .9253 \mathrm{ln}$ $112.54158 .7822 \mathrm{ln}$ $112.66658 .6152 \mathrm{ln}$ $112.80558 .4717 \mathrm{ln}$ $112.9358 .3286 \mathrm{ln}$ $113.0758 .1616 \mathrm{ln}$ $113.19558 .0186 \mathrm{ln}$ $113.32157 .8755 \mathrm{ln}$ $113.4657 .708 \mathrm{ln}$ $113.58657 .5649 \mathrm{ln}$ $113.71157 .4219 \mathrm{ln}$ $113.836 \quad 57.2549 \mathrm{ln}$ $113.96257 .1118 \mathrm{ln}$ $114.10256 .9688 \mathrm{ln}$ $114.22856 .8252 \mathrm{ln}$ $114.35356 .6582 \mathrm{ln}$ $114.478 \quad 56.5151 \mathrm{ln}$ $114.60456 .3721 \mathrm{ln}$ $114.71556 .2051 \mathrm{ln}$ $114.8456 .062 \mathrm{ln}$ $114.96655 .9185 \mathrm{ln}$ $115.09155 .7515 \mathrm{ln}$ $115.218 \quad 55.6084 \mathrm{ln}$ $115.32955 .4653 \mathrm{ln}$ 
$115.455 \quad 55.2983 \mathrm{ln}$ $115.58 \quad 55.1553 \mathrm{ln}$ $115.69255 .0117 \mathrm{ln}$ $115.818 \quad 54.8447 \mathrm{ln}$ 115.92854 .7017 ln $116.05454 .5586 \mathrm{ln}$ $116.16654 .3916 \mathrm{ln}$ $116.278 \quad 54.2485 \mathrm{ln}$ 116.40354 .1055 In $116.51553 .938 \mathrm{ln}$ 116.62653 .7949 In 116.73853 .6519 In $116.84953 .4849 \mathrm{ln}$ 116.97453 .3418 ln 117.08653 .1982 In $117.19853 .0313 \mathrm{ln}$ $117.30952 .8882 \mathrm{ln}$ $117.4252 .7451 \mathrm{ln}$ 117.53252 .5781 In $117.64452 .4351 \mathrm{ln}$ $117.74252 .2915 \mathrm{ln}$ $117.85352 .1245 \mathrm{ln}$ $117.96551 .9814 \mathrm{ln}$ $118.07651 .8384 \mathrm{ln}$ $118.17451 .6714 \mathrm{ln}$ $118.28651 .5283 \mathrm{ln}$ $118.39751 .3853 \mathrm{ln}$ $118.49551 .2178 \mathrm{ln}$ $118.60751 .0747 \mathrm{ln}$ $118.70450 .9316 \mathrm{ln}$ $118.81650 .7646 \mathrm{ln}$ $118.91350 .6216 \mathrm{ln}$ $119.02450 .478 \mathrm{ln}$ $119.12350 .311 \mathrm{ln}$ $119.2250 .168 \mathrm{ln}$ $119.33250 .0249 \mathrm{ln}$ 119.42949 .8579 In $119.527 \quad 49.7148 \mathrm{ln}$ $119.62549 .5713 \mathrm{ln}$ $119.722 \quad 49.4043 \mathrm{ln}$ $119.8249 .2612 \mathrm{ln}$ $119.93149 .1182 \mathrm{ln}$ $120.02948 .9512 \mathrm{ln}$ $120.126 \quad 48.8081 \mathrm{ln}$ $120.224 \quad 48.665 \mathrm{ln}$ $120.30748 .4976 \mathrm{ln}$ $120.406 \quad 48.3545 \mathrm{ln}$ $120.503 \quad 48.2114 \mathrm{ln}$ $120.648 .0444 \mathrm{ln}$ $120.69947 .9014 \mathrm{ln}$ $120.795 \quad 47.7578 \mathrm{ln}$ $120.87947 .5908 \mathrm{ln}$ $120.977 \quad 47.4478$ ln $121.075 \quad 47.3047 \mathrm{ln}$ $121.15947 .1377 \mathrm{ln}$ $121.256 \quad 46.9946 \mathrm{ln}$ $121.3446 .8516 \mathrm{ln}$ 


\begin{tabular}{|c|c|}
\hline 121.437 & \\
\hline 121.521 & $46.541 \mathrm{ln}$ \\
\hline 1.619 & 46.3979 l \\
\hline 1.703 & $46.231 \mathrm{ln}$ \\
\hline .799 & 6.08791 \\
\hline & 5.9443 \\
\hline & .7773 \\
\hline & \\
\hline & 12 \\
\hline & 5.3242 \\
\hline & 45.18121 \\
\hline $4 \quad 45$ & $5.0376 \ln$ \\
\hline & 7.8706 \\
\hline & 44.7275 \\
\hline & 4.5845 \\
\hline 4 & 44.4175 \\
\hline 3 & 44.2744 \\
\hline 16 & 44.1313 \\
\hline & 43.9639 \\
\hline 69 & 43.8208 \\
\hline & 43.6777 \\
\hline 3.237 & 43.51071 \\
\hline 3.324 & $43.3677 \mathrm{ln}$ \\
\hline 3.404 & 43.22411 \\
\hline 3.487 & 43.05711 \\
\hline 3.557 & 42.91411 \\
\hline 3.644 & $42.771 \ln$ \\
\hline 724 & 42.62791 \\
\hline 94 & 42.4609 \\
\hline .878 & 42.3174 \\
\hline & 42.1743 \\
\hline 32 & 42.0073 \\
\hline & 41.8643 \\
\hline & 41.7212 \\
\hline & 41.5542 \\
\hline & 41. \\
\hline & 41.2 \\
\hline & 41.1006 \\
\hline & 40. \\
\hline & 451 \\
\hline & 1751 \\
\hline & 39 \\
\hline & 508 \\
\hline & 938 \\
\hline & 508 \\
\hline & 077 \\
\hline 5.133 & 39.7407 \\
\hline 5 & 39.5977 \\
\hline 25.273 & 39.4541 \\
\hline 25.356 & 39.28711 \\
\hline 25.425 & $39.144 \mathrm{ln}$ \\
\hline 125.482 & $39.001 \mathrm{ln}$ \\
\hline 125.551 & $38.834 \mathrm{ln}$ \\
\hline & 38.69091 \\
\hline 125.691 & 38.54741 \\
\hline & $38.3804 \mathrm{ln}$ \\
\hline 125.83 & $38.2373 \mathrm{ln}$ \\
\hline
\end{tabular}


$125.938 .0942 \mathrm{ln}$ $125.95637 .9272 \mathrm{ln}$ $126.02537 .7837 \mathrm{ln}$ $126.09537 .6406 \mathrm{ln}$ $126.16637 .4736 \mathrm{ln}$ $126.2237 .3306 \mathrm{ln}$ $126.29137 .1875 \mathrm{ln}$ $126.36137 .0205 \mathrm{ln}$ $126.41636 .8774 \mathrm{ln}$ $126.48636 .7339 \mathrm{ln}$ $126.54236 .5669 \mathrm{ln}$ $126.61236 .4238 \mathrm{ln}$ $126.66736 .2808 \mathrm{ln}$ $126.73736 .1138 \mathrm{ln}$ $126.79235 .9707 \mathrm{ln}$ $126.86235 .8271 \mathrm{ln}$ $126.91835 .6602 \mathrm{ln}$ $126.97435 .5171 \mathrm{ln}$ $127.043 \quad 35.374 \quad 1 n$ $127.09935 .207 \mathrm{ln}$ $127.15535 .0635 \mathrm{ln}$ $127.22434 .9204 \mathrm{ln}$ $127.28134 .7534 \mathrm{ln}$ $127.336 \quad 34.6104 \mathrm{ln}$ $127.39234 .4673 \mathrm{ln}$ $127.44834 .3003 \mathrm{ln}$ $127.51734 .1572 \mathrm{ln}$ $127.57434 .0137 \mathrm{ln}$ $127.628 \quad 33.8467 \mathrm{ln}$ $127.68533 .7036 \mathrm{ln}$ $127.74133 .5605 \mathrm{ln}$ $127.79633 .3936 \mathrm{ln}$ $127.85233 .25 \mathrm{ln}$ $127.908 \quad 33.1069$ ln $127.96432 .9399 \mathrm{ln}$ $128.0232 .7969 \mathrm{ln}$ $128.076 \quad 32.6538 \mathrm{ln}$ $128.13132 .4868 \mathrm{ln}$ $128.18732 .3438 \mathrm{ln}$ $128.22932 .2002 \mathrm{ln}$ $128.28532 .0332 \mathrm{ln}$ $128.3431 .8901 \mathrm{ln}$ $128.39631 .7471 \mathrm{ln}$ $128.45331 .5801 \mathrm{ln}$ $128.49431 .437 \mathrm{ln}$ $128.54931 .2935 \mathrm{ln}$ $128.60631 .1265 \mathrm{ln}$ $128.66230 .9834 \mathrm{ln}$ $128.70330 .8403 \mathrm{ln}$ $128.75830 .6733 \mathrm{ln}$ $128.8 \quad 30.5303 \mathrm{ln}$ $128.85730 .3867 \mathrm{ln}$ $128.91230 .2197 \mathrm{ln}$ $128.95430 .0767 \mathrm{ln}$ $129.0129 .9336 \mathrm{ln}$ $129.05229 .7666 \mathrm{ln}$ $129.10729 .6235 \mathrm{ln}$ 


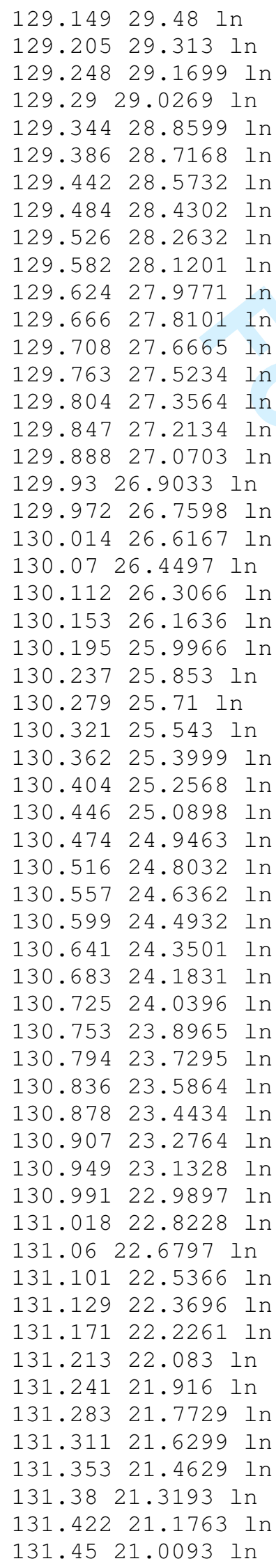


$131.49220 .8662 \mathrm{ln}$

$131.52 \quad 20.7231 \mathrm{ln}$

131.56220 .5562 ln

$131.5920 .4126 \mathrm{ln}$

$131.63120 .2695 \mathrm{ln}$

$131.6620 .1025 \mathrm{ln}$

$131.687 \quad 19.9595 \mathrm{ln}$

$131.7319 .8164 \mathrm{ln}$

$131.757 \quad 19.6494 \mathrm{ln}$

$131.78519 .5059 \mathrm{ln}$

$131.82719 .3628 \mathrm{ln}$

131.85519 .1958 In

$\mathrm{s}$

save_ctm

/Helvetica*1 findfont $\left[\begin{array}{lllllll}6.25 & 0 & 0 & -6.25 & 0 & 0\end{array}\right]$ mfnt sfnt BuildWidthShowArray

$142.52458 .6865 \mathrm{mov}$

(1) $\mathrm{sh}$

/Helvetica*3 findfont $\left[\begin{array}{llllll}6.25 & 0 & 0 & -6.25 & 0 & 0\end{array}\right] \mathrm{mfnt}$ sfnt BuildWidthShowArray

$145.99958 .6865 \mathrm{mov}$

(5) $\mathrm{sh}$

/Helvetica*2 findfont $\left[\begin{array}{lllllll}6.25 & 0 & 0 & -6.25 & 0 & 0\end{array}\right] \mathrm{mfnt}$ sfnt

BuildWidthShowArray

$149.47458 .6865 \mathrm{mov}$

(00) $\mathrm{sh}$

restore_ctm

save_ctm

ADOBeginSubsetFont: Helvetica AddGlyphs

systemdict begin

/Helvetica

findfont dup

/Private get begin

/CharStrings get begin

/space

$<10$ bf 317079c7734bf7> ND

end end

end

/Helvetica findfont /Encoding get

dup 32 /space put

pop

ADOEndSubsetFont

/Helvetica*4

[

$32\{/$.notdef\}repeat /space $223\{/$.notdef\}repeat

] /Helvetica nfnt

/Helvetica*4 findfont $\left[\begin{array}{lllllll}0 & -7.75 & -7.75 & 0 & 0 & 0\end{array}\right]$ mfnt sfnt

BuildWidthShowArray

$165.79691 .7349 \mathrm{mov}$

( ) $\mathrm{sh}$

restore_ctm

$0.5 \mathrm{lw}$

$134.778 \quad 18.7969 \mathrm{mo}$

$136.9816 .583 \mathrm{ln}$

$139.10218 .6943 \mathrm{ln}$

$\mathrm{S}$

URL: http://mc.manuscriptcentral.com/tandf/tmph 


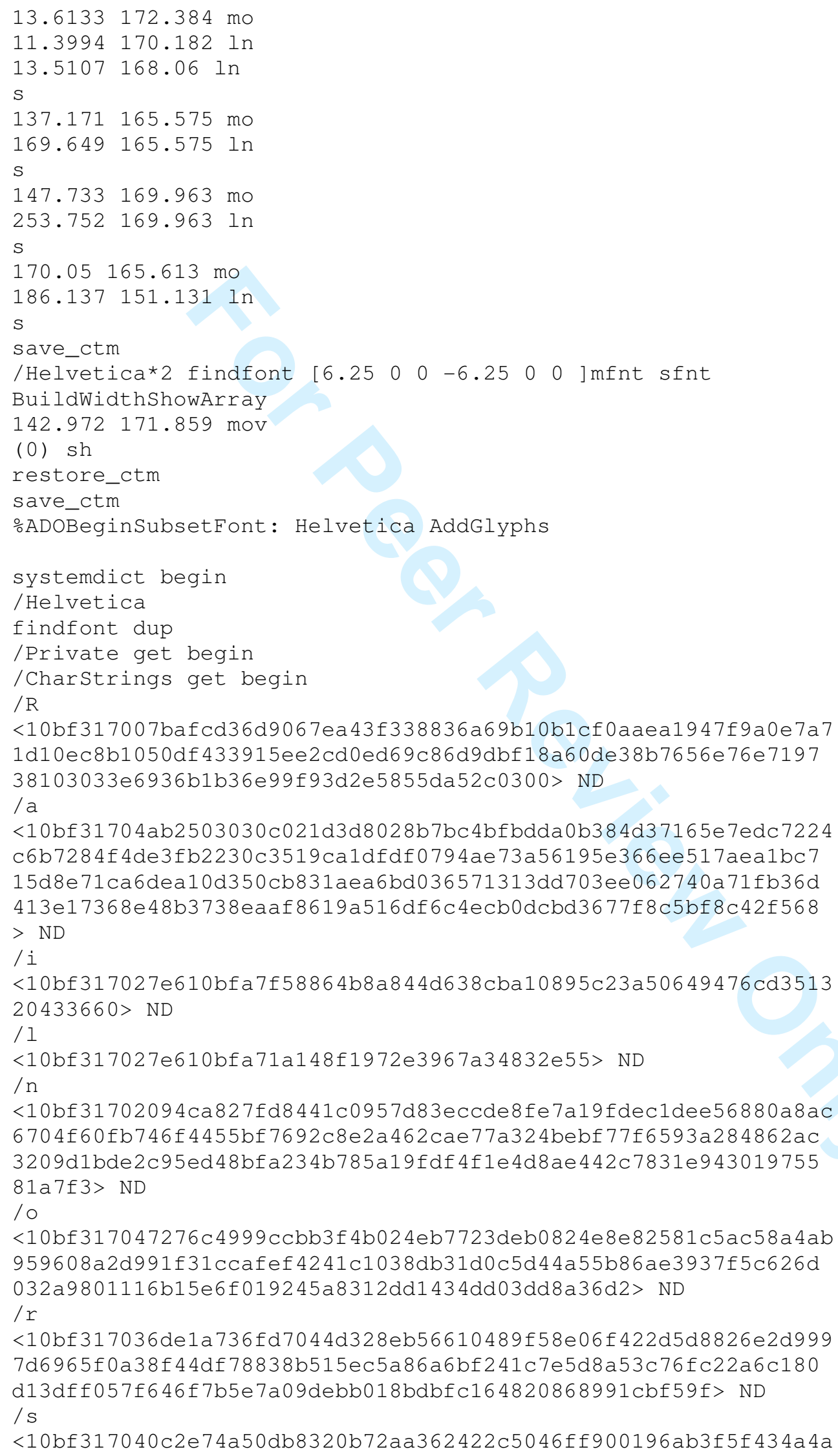

URL: http://mc.manuscriptcentral.com/tandf/tmph 
ef605ac2e8787b3b2c0a32f8140a 7caf48f682c792596170e829a 74 eeab9 e 6 b13e51 72982e4c3aa3bd41b0f2649cd6fc 7558007 ffe3d8b95c3a53b15 d60552dc9d8b58f17081fcda3d7441e8da35b81ca90071d9a58c33330a6c c65f543fd95a38eac5bb4 70b69332ac4e09a31d6ff0c3c6f7132ba18b413 e71f969364b63412c18b670d7baef70c500eb778e76e> ND 
$197.75166 .946 \mathrm{mov}$

(o) $\mathrm{sh}$

\%ADOBeginSubsetFont: Helvetica AddGlyphs

systemdict begin

/ Helvetica

findfont dup

/Private get begin

/Charstrings get begin

/ C

<10bf317041f0a949233299062f20927d17046b803a3239107f5fc5937643

$6 \mathrm{~d} 193872 \mathrm{a} 9 \mathrm{c} 41376664$ ee1d53646aab55191f7f22c2d93139e9b3f1f 9218

d273604ce5dbbf3c0707849e2a0760> ND

/e

$<10$ bf 31704 ab2503030fec 2 f 45 e 7 e 5 cf 47 e903e6 74 aa 3389 f 3620374 e 8 c 9 e d34a92ac68abe08c5fb 790 c6003e9d3a6d07d3d1b09583c781dacd 978778

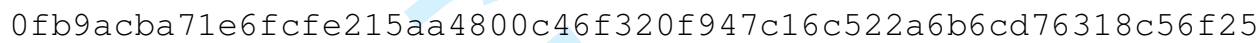
e3703c6a5c41576b7f6c890695b9841c9ec2> ND

$/ \mathrm{f}$

<10bf31706fd71d386b83230daddba58a9e6370f 44 bacd52b1 729701425 a3 043058 b817c8fb50597ee8d81edc0a37a40b7e19b23074ce2d363afa5ee 9 $6552508937 \mathrm{dc} 332 \mathrm{c} 9 \mathrm{dd} 4 \mathrm{e} 8 \mathrm{~b} 3 \mathrm{c} 28 \mathrm{e} 57028 \mathrm{~b} 5 \mathrm{f} 6 \mathrm{~b} 663 \mathrm{be} 18248199 \mathrm{~b} 1 \mathrm{c} 2918 \mathrm{~b} 8$ $051 \mathrm{~d} 7 \mathrm{~d} 1 \mathrm{a} 830 \mathrm{acbce} 6746>\mathrm{ND}$

$/ \mathrm{h}$

$<10$ bf 31702094 ca 827 fd8441c0957d83eccde 8 fe $7 a 19$ fdecldee 56880 a 8 ac 6704 f60fb746f4455bf 7692 c80e4dd6f 72689 feldfcf 08 b 95 d 9 d2 7 b6 29 c 9 6 be 320675 c 7 f 0 d2 2 ac1 909 addc 91 e 4 d 24 f 538497 c $3 a 125$ f 2290 f 2 e 33 a 5 c 0 $34095 \mathrm{C} 76>\mathrm{ND}$

$/ \mathrm{m}$

$<10$ bf 317021 c2695e9c7fa6f82eed1619e9e5c441345edc34908a1558c814 d098cad2a350950c603d48fbbf 1 dd3892ba3a 7 a 0 f 1377 b 46 e 09 ac 5 cade 6 a $435 \mathrm{db} 726 \mathrm{ce} 747 \mathrm{dab} 69 \mathrm{cfed} 4 \mathrm{ab} 554 \mathrm{ba} 84052$ e66fc7e $07266 \mathrm{c} 9817537 \mathrm{~d} 65 \mathrm{bf}$ f 31 b2 4021 df 986002 b 731 bd 41 bbf 2101 ea 82 a 479977265 b 6 a 16 df de 920 b0 $69054 \mathrm{a} 15036 \mathrm{~b} 7 \mathrm{~d} 78794 \mathrm{~b} 70 \mathrm{c} 9 \mathrm{c} 5 \mathrm{ec} 866 \mathrm{aa} 5647 \mathrm{a} 925 \mathrm{c} 21749 \mathrm{bf} 38329 \mathrm{c} 50848$ b6eafd5abbaf882d0f9ce6> ND

$/ \mathrm{u}$

<10bf31703f932f5ed018c596aaa 8 f 7609375 f318f 4 ecbec66e97863e 3512 ff20661d34a 4e877adb4c4b5c4a6d91847b1377efecd3a8443467ed2398e c 773d19d50f0 765cla 8c0f3f7a31fd382b41fddd16b9df52d173511bb8dd $7 \mathrm{C}>\mathrm{ND}$

/ v

$<10$ bf 317069 b1fd714631eca10c760070117668a0c05e5b38c7d861f456c0 e3dd6317ac8060fdad17b6a854b5db0fcf934a34a5a2> ND

$/ \mathrm{y}$ <10bf3170789a7df8aefa30c3938f228b6b52130014271654504f35238741 a 244043537 fe 890 efa 6389032 d5a 401 fa 6 e2966e86999b879839803ea 716 e946819819ecb365bc221200103f4a35d4583ace0d158a40057484793585 990 bda1661163ff61d7709d6c92810ca8ead63d8> ND

end end

end

/Helvetica findfont /Encoding get

dup 99 / c put

dup 101 /e put

dup 102 /f put

dup $104 / \mathrm{h}$ put

dup $109 / \mathrm{m}$ put

dup $117 /$ u put

URL: http://mc.manuscriptcentral.com/tandf/tmph 
dup $118 / \mathrm{v}$ put

dup $121 /$ y put

pop

ADOEndSubsetFont

/Helvetica*6

[

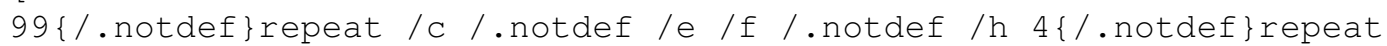

$/ \mathrm{m} 7\{/$.notdef\}repeat /u /v 2\{/.notdef\}repeat /y 134\{/.notdef\}repeat

] /Helvetica nfint

/Helvetica*6 findfont $\left[\begin{array}{lllllll}6.25 & 0 & 0 & -6.25 & 0 & 0\end{array}\right]$ mfnt sfnt

BuildWidthShowArray

$201.225166 .946 \mathrm{mov}$

(f) $\mathrm{sh}$

/Helvetica*4 findfont $\left[\begin{array}{lllllll}6.25 & 0 & 0 & -6.25 & 0 & 0\end{array}\right] \mathrm{mfnt}$ sfnt

BuildWidthShowArray

$202.962166 .946 \mathrm{mov}$

( ) sh

/ Helvetica*6 findfont $\left[\begin{array}{lllllll}6.25 & 0 & 0 & -6.25 & 0 & 0\end{array}\right] \mathrm{mfnt}$ sfnt BuildWidthShowArray

204.7166 .946 mov

(he) sh

/Helvetica*5 findfont $\left[\begin{array}{lllllll}6.25 & 0 & 0 & -6.25 & 0 & 0\end{array}\right]$ mfnt sfnt

BuildWidthShowArray

$211.65166 .946 \mathrm{mov}$

(a) $\mathrm{sh}$

/Helvetica*6 findfont $\left[\begin{array}{llllll}6.25 & 0 & 0 & -6.25 & 0 & 0\end{array}\right]$ mfnt sfnt

BuildWidthShowArray

$215.124166 .946 \mathrm{mov}$

(vy) sh

/Helvetica*4 findfont $\left[\begin{array}{llllll}6.25 & 0 & 0 & -6.25 & 0 & 0\end{array}\right]$ mfnt sfnt BuildWidthShowArray

$221.374166 .946 \mathrm{mov}$

( ) sh

/Helvetica*6 findfont $\left[\begin{array}{lllllll}6.25 & 0 & 0 & -6.25 & 0 & 0\end{array}\right] \mathrm{mfnt}$ sfnt BuildWidthShowArray

$223.112166 .946 \mathrm{mov}$

(m) $\mathrm{sh}$

/Helvetica*5 findfont $\left[\begin{array}{lllllll}6.25 & 0 & 0 & -6.25 & 0 & 0\end{array}\right]$ mfnt sfnt

BuildWidthShowArray

$228.318166 .946 \mathrm{mov}$

(ol) $\mathrm{sh}$

/Helvetica*6 findfont $\left[\begin{array}{lllllll}6.25 & 0 & 0 & -6.25 & 0 & 0\end{array}\right]$ mfnt sfnt BuildWidthShowArray

$233.181166 .946 \mathrm{mov}$

(ecu) sh

/Helvetica*5 findfont $\left[\begin{array}{lllllll}6.25 & 0 & 0 & -6.25 & 0 & 0\end{array}\right] \mathrm{mfnt}$ sfnt BuildWidthShowArray

$243.256166 .946 \mathrm{mov}$

(1) $\mathrm{sh}$

/Helvetica*6 findfont $\left[\begin{array}{lllllll}6.25 & 0 & 0 & -6.25 & 0 & 0\end{array}\right] \mathrm{mfnt}$ sfnt BuildWidthShowArray

$244.643166 .946 \mathrm{mov}$

(e) $\mathrm{sh}$

/Helvetica*5 findfont $\left[\begin{array}{lllllll}6.25 & 0 & 0 & -6.25 & 0 & 0\end{array}\right]$ mfnt sfnt

BuildwidthShowArray

$248.118166 .946 \mathrm{mov}$

( s) $\mathrm{sh}$

URL: http://mc.manuscriptcentral.com/tandf/tmph 
$103\{/$.notdef $\}$ repeat /g $152\{/$. notdef $\}$ repeat

] /Helvetica nfnt

/Helvetica*7 findfont $\left[\begin{array}{lllllll}6.25 & 0 & 0 & -6.25 & 0 & 0\end{array}\right]$ mfnt sfnt BuildWidthShowArray

$212.348147 .893 \mathrm{mov}$

(g) $\mathrm{sh}$

/Helvetica*6 findfont $\left[\begin{array}{lllllll}6.25 & 0 & 0 & -6.25 & 0 & 0\end{array}\right]$ mfnt sfint BuildwidthShowArray

$215.823147 .893 \mathrm{mov}$

(h) $\mathrm{sh}$

/Helvetica*5 findfont $\left[\begin{array}{lllllll}6.25 & 0 & 0 & -6.25 & 0 & 0\end{array}\right]$ mfnt sfnt BuildWidthShowArray

$219.298147 .893 \mathrm{mov}$

(t) $\mathrm{sh}$

/Helvetica*4 findfont $\left[\begin{array}{lllllll}6.25 & 0 & 0 & -6.25 & 0 & 0\end{array}\right]$ mfnt sfnt BuildWidthShowArray

$221.036147 .893 \mathrm{mov}$

( ) $\mathrm{sh}$

/Helvetica*6 findfont $\left[\begin{array}{llllll}6.25 & 0 & 0 & -6.25 & 0 & 0\end{array}\right]$ mfnt sfnt BuildWidthShowArray

$222.773147 .893 \mathrm{mov}$

(m) $\mathrm{sh}$

/Helvetica*5 findfont $\left[\begin{array}{lllllll}6.25 & 0 & 0 & -6.25 & 0 & 0\end{array}\right]$ mfnt sfnt BuildWidthShowArray

$227.98147 .893 \mathrm{mov}$

(ol) sh

/Helvetica*6 findfont $\left[\begin{array}{lllllll}6.25 & 0 & 0 & -6.25 & 0 & 0\end{array}\right] \mathrm{mfnt}$ sfnt BuildWidthShowArray $232.842147 .893 \mathrm{mov}$ (ecu) sh /Helvetica*5 findfont $\left[\begin{array}{llllll}6.25 & 0 & 0 & -6.25 & 0 & 0\end{array}\right]$ mfnt sfnt BuildWidthShowArray

$242.917147 .893 \mathrm{mov}$

(1) $\mathrm{sh}$

/Helvetica*6 findfont $\left[\begin{array}{llllll}6.25 & 0 & 0 & -6.25 & 0 & 0\end{array}\right]$ mfnt sfint BuildWidthShowArray

$244.304147 .893 \mathrm{mov}$

(e) $\mathrm{sh}$

/ Helvetica*5 findfont $\left[\begin{array}{lllllll}6.25 & 0 & 0 & -6.25 & 0 & 0\end{array}\right] \mathrm{mfnt}$ sfnt BuildwidthShowArray

$247.779147 .893 \mathrm{mov}$

(s) $\mathrm{sh}$

restore_ctm

save_ctm

ADOBeginSubsetFont: Helvetica AddGlyphs

systemdict begin

/Helvetica

findfont dup

/Private get begin

/CharStrings get begin

/V

$<10$ bf 3170573 f 824 b12583a5c22b8f9dfe20065e831d5da166a512eb722d5

$028 f 1679 c 64 c 43 c 669716$ c5d9a6beb31a1882e0959cd84893d98> ND

$/ \mathrm{b}$

$<10$ bf 31702094 ca820c254a 78deec33d46bcefb3b88be60ae4a539cc46302

cfc62a03dc640b42f99a02da9a 79950b22a9d589cc8ecdf 47 aad59a18d38

URL: http://mc.manuscriptcentral.com/tandf/tmph 


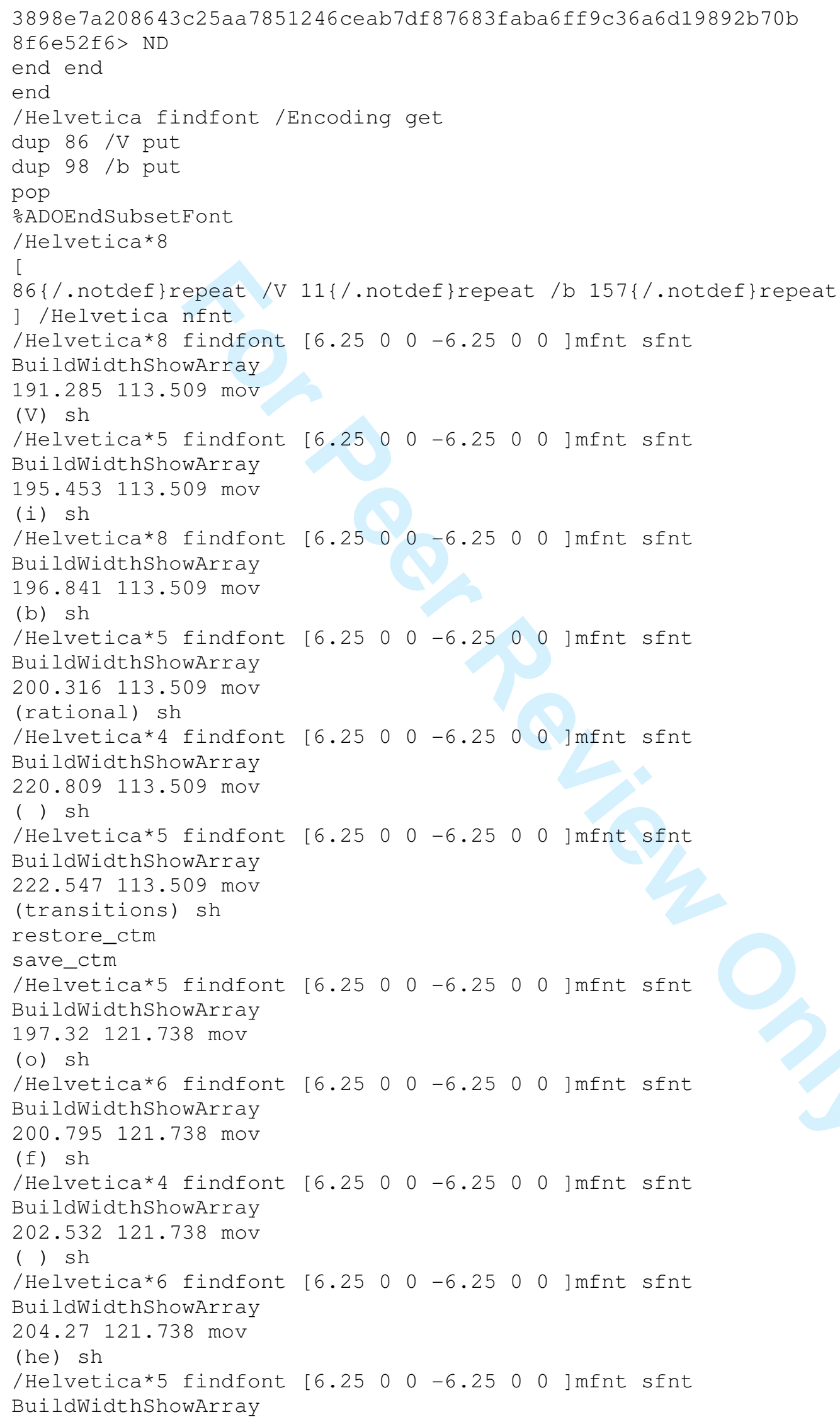


$211.22121 .738 \mathrm{mov}$

(a) sh

/ Helvetica*6 findfont $\left[\begin{array}{lllllll}6.25 & 0 & 0 & -6.25 & 0 & 0\end{array}\right]$ mfnt sfnt BuildWidthShowArray

$214.695121 .738 \mathrm{mov}$

(vy) sh

/Helvetica*4 findfont $\left[\begin{array}{llllllll}6.25 & 0 & 0 & -6.25 & 0 & 0\end{array}\right]$ mfnt sfnt BuildWidthShowArray $220.945121 .738 \mathrm{mov}$ ( ) sh

/ Helvetica*6 findfont $\left[\begin{array}{lllllll}6.25 & 0 & 0 & -6.25 & 0 & 0\end{array}\right]$ mfnt sfnt BuildWidthShowArray $222.682121 .738 \mathrm{mov}$ (m) sh / Helvetica*5 findfont $\left[\begin{array}{lllllll}6.25 & 0 & 0 & -6.25 & 0 & 0\end{array}\right]$ mfnt sfnt BuildWidthShowArray $227.889121 .738 \mathrm{mov}$ ( 01 ) $\mathrm{sh}$

/Helvetica*6 findfont $\left[\begin{array}{lllllll}6.25 & 0 & 0 & -6.25 & 0 & 0\end{array}\right]$ mfnt sfnt BuildWidthShowArray $232.751121 .738 \mathrm{mov}$ (ecu) sh /Helvetica*5 findfont $\left[\begin{array}{lllllll}6.25 & 0 & 0 & -6.25 & 0 & 0\end{array}\right] \mathrm{mfnt}$ sfnt BuildwidthShowArray $242.826121 .738 \mathrm{mov}$ (1) $\mathrm{sh}$ / Helvetica*6 findfont $\left[\begin{array}{llllll}6.25 & 0 & 0 & -6.25 & 0 & 0\end{array}\right]$ mfnt sfnt BuildWidthShowArray $244.213121 .738 \mathrm{mov}$ (e) $\mathrm{sh}$ / Helvetica*5 findfont $\left[\begin{array}{lllllll}6.25 & 0 & 0 & -6.25 & 0 & 0\end{array}\right]$ mfnt sfnt BuildWidthShowArray $247.688121 .738 \mathrm{mov}$ (s) $\mathrm{sh}$ 
BuildWidthShowArray $215.86774 .6011 \mathrm{mov}$

(h) sh

/ Helvetica*5 findfont $\left[\begin{array}{lllllll}6.25 & 0 & 0 & -6.25 & 0 & 0\end{array}\right]$ mfnt sfnt BuildWidthShowArray

$219.34274 .6011 \mathrm{mov}$

(t) $\mathrm{sh}$

/Helvetica*4 findfont $\left[\begin{array}{lllllll}6.25 & 0 & 0 & -6.25 & 0 & 0\end{array}\right] \mathrm{mfnt}$ sfnt BuildWidthShowArray

$221.0874 .6011 \mathrm{mov}$

( ) $\mathrm{sh}$

/Helvetica*6 findfont $\left[\begin{array}{lllllll}6.25 & 0 & 0 & -6.25 & 0 & 0\end{array}\right]$ mfnt sfnt BuildwidthShowArray

$222.81774 .6011 \mathrm{mov}$

(m) sh

/ Helvetica*5 findfont $\left[\begin{array}{llllll}6.25 & 0 & 0 & -6.25 & 0 & 0\end{array}\right]$ mfnt sfnt

BuildwidthShowArray

$228.02374 .6011 \mathrm{mov}$

(ol) $\mathrm{sh}$

/Helvetica*6 findfont $\left[\begin{array}{lllllll}6.25 & 0 & 0 & -6.25 & 0 & 0\end{array}\right]$ mfnt sfnt BuildWidthShowArray

$232.88674 .6011 \mathrm{mov}$

(ecu) sh

/Helvetica*5 findfont [ $\left[\begin{array}{llllll}6.25 & 0 & 0 & -6.25 & 0 & 0\end{array}\right]$ mfnt sfnt BuildWidthShowArray

$242.96174 .6011 \mathrm{mov}$

(1) $\mathrm{sh}$

/Helvetica*6 findfont $\left[\begin{array}{lllllll}6.25 & 0 & 0 & -6.25 & 0 & 0\end{array}\right]$ mfnt sfnt BuildWidthShowArray

$244.34874 .6011 \mathrm{mov}$

(e) $\mathrm{sh}$

/ Helvetica*5 findfont $\left[\begin{array}{lllllll}6.25 & 0 & 0 & -6.25 & 0 & 0\end{array}\right] \mathrm{mfnt}$ sfnt BuildWidthShowArray

$247.82374 .6011 \mathrm{mov}$

(s) $\mathrm{sh}$

restore_ctm

save_ctm

/Helvetica*8 findfont $\left[\begin{array}{lllllll}6.25 & 0 & 0 & -6.25 & 0 & 0\end{array}\right]$ mfnt sfnt

BuildWidthShowArray

$190.96866 .8569 \mathrm{mov}$

(V) sh

/ Helvetica*5 findfont $\left[\begin{array}{llllllll}6.25 & 0 & 0 & -6.25 & 0 & 0\end{array}\right]$ mfnt sfnt BuildWidthShowArray

$195.13766 .8569 \mathrm{mov}$

(i) $\mathrm{sh}$

/ Helvetica*8 findfont $\left[\begin{array}{lllllll}6.25 & 0 & 0 & -6.25 & 0 & 0\end{array}\right] \mathrm{mfnt}$ sfnt BuildWidthShowArray

$196.52466 .8569 \mathrm{mov}$

(b) $\mathrm{sh}$

/ Helvetica*5 findfont $\left[\begin{array}{lllllll}6.25 & 0 & 0 & -6.25 & 0 & 0\end{array}\right]$ mfnt sfnt BuildWidthShowArray

$199.99966 .8569 \mathrm{mov}$

(rational) sh

/ Helvetica*4 findfont $\left[\begin{array}{lllllll}6.25 & 0 & 0 & -6.25 & 0 & 0\end{array}\right]$ mfnt sfnt

BuildWidthShowArray

$220.49366 .8569 \mathrm{mov}$

( ) sh 


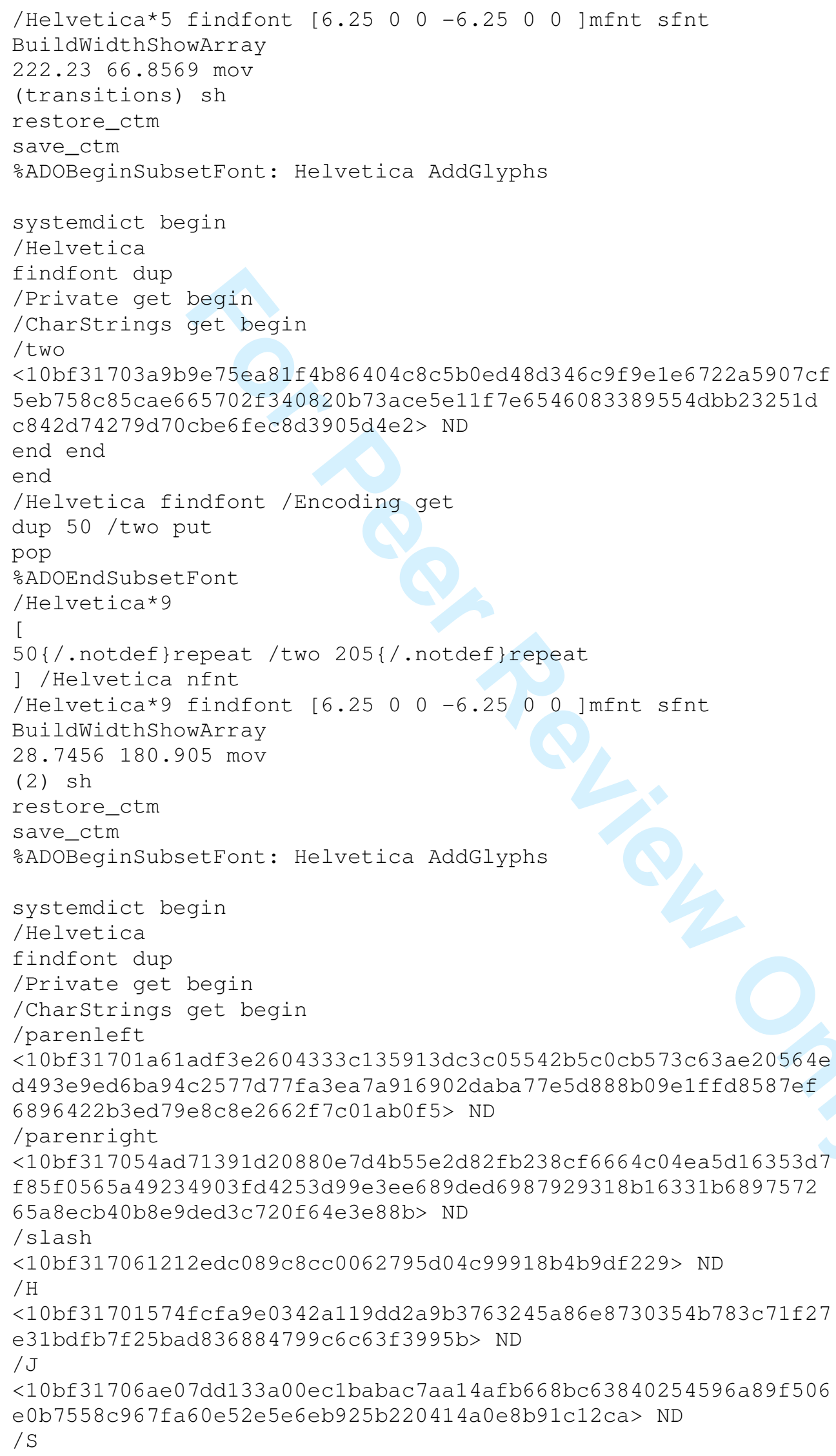


$<10$ bf 317033474622541600 cc2acc0f9a1a21d860dd13cae6b2a2e0d1db84 bf 8 c1 1 b 9 c 8200 d539d5d6df9ad 95 df 75 cbb 91 cee 52542 ba 8 b2e 89 d 06 d 385 $5 \mathrm{f} 6188883 \mathrm{ac} 9 \mathrm{bbacd} 5 \mathrm{c} 45 \mathrm{a} 12 \mathrm{eb} 260272 \mathrm{e} 8 \mathrm{~b} 0 \mathrm{~d} 364 \mathrm{a} 7 \mathrm{ad} 65265 \mathrm{e} 472633926 \mathrm{f}$ e938aace 9 a38 8 efe 95 d9c 448 e 82 e19b19326f278fd8 80 ec 47 df 55 e 79 c 728 96119 b424b1d449136811125c288f0b6f349b2005c3d5c6d67a 814 fb6c 91 c855aa47d559fb4ea193> ND

$/ d$ $<10$ bf 317047276 c4999a66a 7f91a435f5a2bcb493402c2f337c6cef 41543c 564 d2b6989b1943e8fd4c28f55b8e5efc 85 e 71 de 1 caae 3 a 017 bed 9 f c 6 b 70 afba1bfd24a2 7b96bb7a1facf3da 7bfe50e6a 042548913 a 52 e 71105 f 7526 30b5196211a132> ND

$/ \mathrm{p}$ <10bf31702094ca827c0b31e3f26978ae02e 7e4c0f36b1b5615f1b414c499 e $50702 \mathrm{ddb} 3372392561 \mathrm{dbc} 0$ afed $06 \mathrm{ab} 340321 \mathrm{cb} 8 \mathrm{e} 542 \mathrm{cca} 7 \mathrm{fb} 5 \mathrm{de} 8 \mathrm{c} 74274$ 84 ef 63 ea $77040 \mathrm{~d} 0$ ffc 2 ec 6456 dbod 6620 e 2 f 71662 e 8 de 73 a 841 e 0 dcca 864 $520 \mathrm{ce} 883 \mathrm{be} 8257 \mathrm{aa} 7 \mathrm{e} 4646 \mathrm{~d} 5 \mathrm{fe} 5 \mathrm{fcf} 79 \mathrm{a} 1791 \mathrm{bef} 781 \mathrm{a} 08056563 \mathrm{e} 345 \mathrm{c} 07 \mathrm{f}$ 3b8fe8074841eeff0d29dd36d0eabe 46db8114db4f295182> ND

$/ \mathrm{z}$ <10bf31704ab218185f94615d219391c6102bb84a292d20406ae334338215 476329 e 7 dca9973db7d91cd833d1cf> ND end end end /Helvetica findfont / Encoding get dup 40 /parenleft put dup 41 /parenright put dup 47 /slash put dup $72 / \mathrm{H}$ put dup $74 / \mathrm{J}$ put dup $83 / \mathrm{s}$ put dup $100 / d$ put dup $112 / \mathrm{p}$ put dup $122 / z$ put pop \%ADOEndSubsetFont / Helvetica*10 [ $40\{/$.notdef\}repeat /parenleft /parenright 5\{/.notdef\}repeat / slash $24\{/$.notdef $\}$ repeat / H / notdef /J $8\{/$.notdef $\}$ repeat /S $16\{/$.notdef\}repeat /d $11\{/$.notdef $\}$ repeat /p $9\{/$. notdef $\}$ repeat

/z $133\{/$. notdef $\}$ repeat

] /Helvetica nfint / Helvetica*10 findfont [ $\left[\begin{array}{lllllll}6.25 & 0 & 0 & -6.25 & 0 & 0\end{array}\right] \mathrm{mfnt}$ sfnt BuildWidthShowArray

$0188.982 \mathrm{mov}$

( $\mathrm{Sp}) \mathrm{sh}$

/Helvetica*6 findfont $\left[\begin{array}{lllllll}6.25 & 0 & 0 & -6.25 & 0 & 0\end{array}\right]$ mfnt sfnt BuildWidthShowArray $7.64369188 .982 \mathrm{mov}$ (ec) $\mathrm{sh}$ /Helvetica*5 findfont $\left[\begin{array}{lllllll}6.25 & 0 & 0 & -6.25 & 0 & 0\end{array}\right] \mathrm{mfnt}$ sfnt BuildWidthShowArray $14.2437188 .982 \mathrm{mov}$ (tral) sh / Helvetica*4 findfont [ $\left[\begin{array}{llllll}6.25 & 0 & 0 & -6.25 & 0 & 0\end{array}\right] \mathrm{mfnt}$ sfnt BuildWidthShowArray $22.9248188 .982 \mathrm{mov}$ 
( ) sh

/Helvetica*6 findfont $\left[\begin{array}{lllllll}6.25 & 0 & 0 & -6.25 & 0 & 0\end{array}\right]$ mfnt sfnt BuildWidthShowArray

$24.6623188 .982 \mathrm{mov}$

(e) $\mathrm{sh}$

/ Helvetica*5 findfont $\left[\begin{array}{lllllll}6.25 & 0 & 0 & -6.25 & 0 & 0\end{array}\right] \mathrm{mfnt}$ sfnt BuildWidthShowArray

$28.1373188 .982 \mathrm{mov}$

(n) sh

/Helvetica*6 findfont $\left[\begin{array}{llllll}6.25 & 0 & 0 & -6.25 & 0 & 0\end{array}\right]$ mfnt sfnt BuildWidthShowArray

$31.6123188 .982 \mathrm{mov}$

(e) $\mathrm{sh}$

/Helvetica*5 findfont $\left[\begin{array}{llllll}6.25 & 0 & 0 & -6.25 & 0 & 0\end{array}\right]$ mfnt sfnt BuildWidthShowArray

$35.0873188 .982 \mathrm{mov}$

(r) $\mathrm{sh}$

/Helvetica*7 findfont $\left[\begin{array}{lllllll}6.25 & 0 & 0 & -6.25 & 0 & 0\end{array}\right] \mathrm{mfnt}$ sfnt BuildWidthShowArray

$37.1685188 .982 \mathrm{mov}$

(g) $\mathrm{sh}$

/Helvetica*6 findfont $\left[\begin{array}{lllllll}6.25 & 0 & 0 & -6.25 & 0 & 0\end{array}\right] \mathrm{mfnt}$ sfnt BuildWidthShowArray

$40.6435188 .982 \mathrm{mov}$

(y) $\mathrm{sh}$

/Helvetica*4 findfont $\left[\begin{array}{llllll}6.25 & 0 & 0 & -6.25 & 0 & 0\end{array}\right]$ mfnt sfnt

BuildWidthShowArray

$43.7685188 .982 \mathrm{mov}$

( ) sh

/Helvetica*10 findfont $\left[\begin{array}{llllll}6.25 & 0 & 0 & -6.25 & 0 & 0\end{array}\right] \mathrm{mfnt}$ sfnt BuildWidthShowArray

$45.506188 .982 \mathrm{mov}$

(d) $\mathrm{sh}$

/ Helvetica*6 findfont $\left[\begin{array}{lllllll}6.25 & 0 & 0 & -6.25 & 0 & 0\end{array}\right]$ mfnt sfnt BuildWidthShowArray

$48.981188 .982 \mathrm{mov}$

(e) $\mathrm{sh}$

/Helvetica*5 findfont $\left[\begin{array}{llllll}6.25 & 0 & 0 & -6.25 & 0 & 0\end{array}\right]$ mfnt sfnt

BuildWidthShowArray

$52.456188 .982 \mathrm{mov}$

(nsit) sh

/Helvetica*6 findfont $\left[\begin{array}{llllll}6.25 & 0 & 0 & -6.25 & 0 & 0\end{array}\right]$ mfnt sfnt BuildWidthShowArray

$62.1809188 .982 \mathrm{mov}$

(y) $\mathrm{sh}$

/Helvetica*4 findfont $\left[\begin{array}{lllllll}6.25 & 0 & 0 & -6.25 & 0 & 0\end{array}\right] \mathrm{mfnt}$ sfnt

BuildWidthShowArray

$65.3059188 .982 \mathrm{mov}$

( ) $\mathrm{sh}$

/Helvetica*10 findfont $\left[\begin{array}{lllllll}6.25 & 0 & 0 & -6.25 & 0 & 0\end{array}\right]$ mfnt sfnt BuildWidthShowArray

$67.0434188 .982 \mathrm{mov}$

$(\backslash()$ sh

/Helvetica*1 findfont $\left[\begin{array}{lllllll}6.25 & 0 & 0 & -6.25 & 0 & 0\end{array}\right]$ mfnt sfnt

BuildWidthShowArray

$69.1246188 .982 \mathrm{mov}$

(1) $\mathrm{sh}$ 


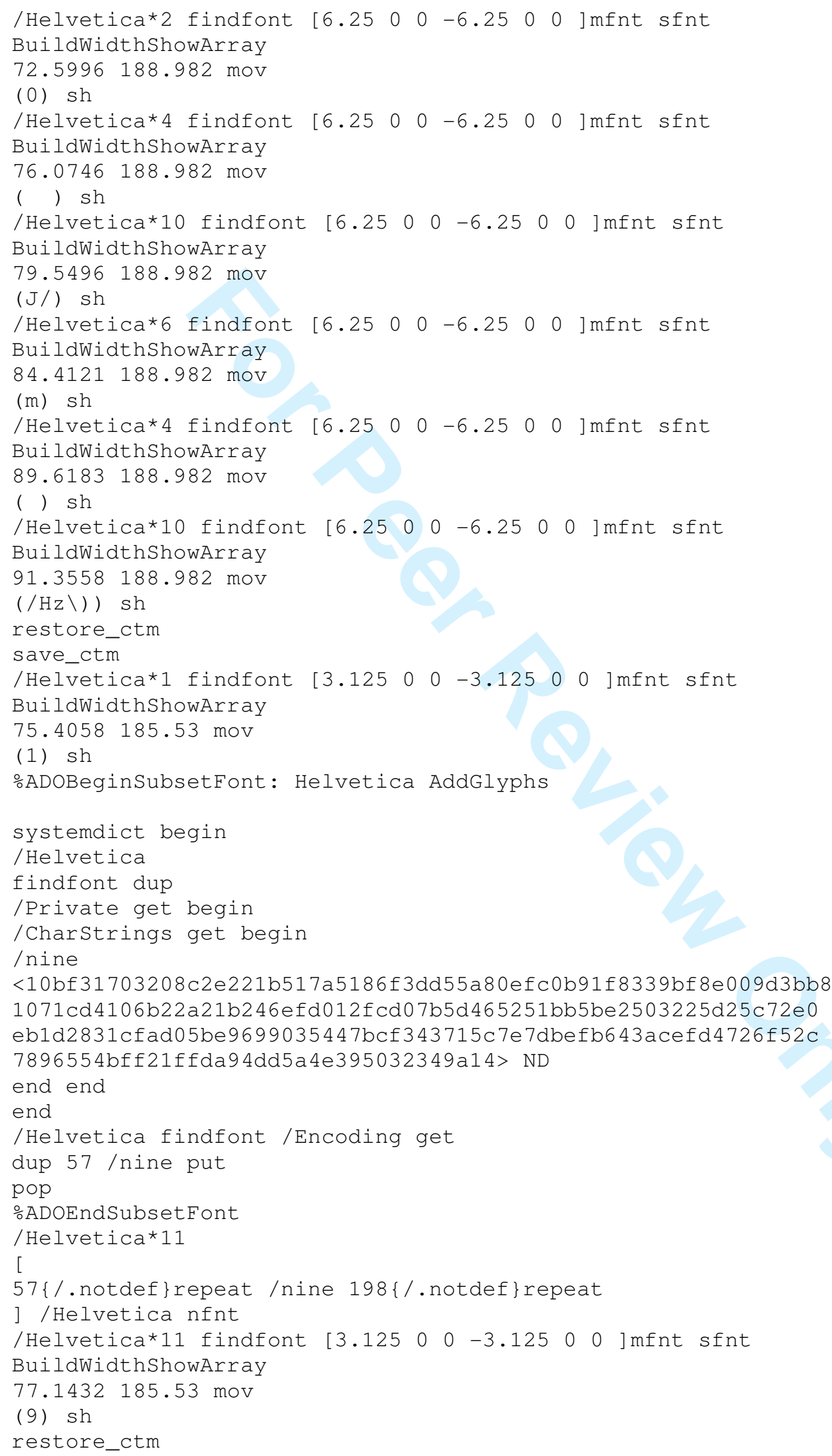


save_ctm

\%ADOBeginsubsetFont: Helvetica AddGlyphs

systemdict begin

/ Helvetica

findfont dup

/Private get begin

/Charstrings get begin

/three

$<10$ bf 31703 e6 5 fd 7e384 78bb8cdc815f391da1d8e98fb667ae1de5387b9e2

5 adf 16 a18c21547c5b7e4a4d3bb688955858c2a2587f 884949 e50417a 4ed da 8 e91b48b3d6aefaa61a288b6b8 877 c330f52739b732112e18c9e2584 77 a11af5a92cb3ed0ed0b61758427ed7a13137d28d15> ND

end end

end

/ Helvetica findfont / Encoding get

dup 51 /three put

pop

ADOEndSubsetFont

/Helvetica*12

[

51 //.notdef $\}$ repeat / three $204\{/$. notdef $\} r e p e a t$

] /Helvetica nfit

/ Helvetica*12 findfont [ $\left[\begin{array}{llllll}3.125 & 0 & 0 & -3.125 & 0 & 0\end{array}\right]$ mfnt sfnt

BuildWidthShowArray

$89.813185 .267 \mathrm{mov}$

(3) $\mathrm{sh}$

restore_ctm

save_ctm

ADOBeginSubsetFont: Helvetica AddGlyphs

systemdict begin

/ Helvetica

findfont dup

/Private get begin

/Charstrings get begin

$/ \mathrm{E}$

$<10$ bf $317005 a 58 e 2868$ ec 80 f $87 b 86200583708$ f 825 d6b2c1c1312977a2aa0

9136 ca794a38c61a48ea76846c0f02d850d7a99e> ND

end end

end

/Helvetica findfont / Encoding get

dup 69 /E put

pop

ADOEndSubsetFont

/ Helvetica*13

[

$69\{/$.notdef $\}$ repeat /E $186\{/$. notdef $\}$ repeat

] /Helvetica nfnt

/ Helvetica*13 findfont $\left[\begin{array}{lllllll}6.25 & 0 & 0 & -6.25 & 0 & 0\end{array}\right]$ mfnt sfnt

BuildWidthShowArray

$102.4813 .4912 \mathrm{mov}$

(E) $\mathrm{sh}$

/ Helvetica*5 findfont $\left[\begin{array}{llllll}6.25 & 0 & 0 & -6.25 & 0 & 0\end{array}\right]$ mfnt sfnt

BuildWidthShowArray

$106.64913 .4912 \mathrm{mov}$

(n) $\mathrm{sh}$

URL: http://mc.manuscriptcentral.com/tandf/tmph 


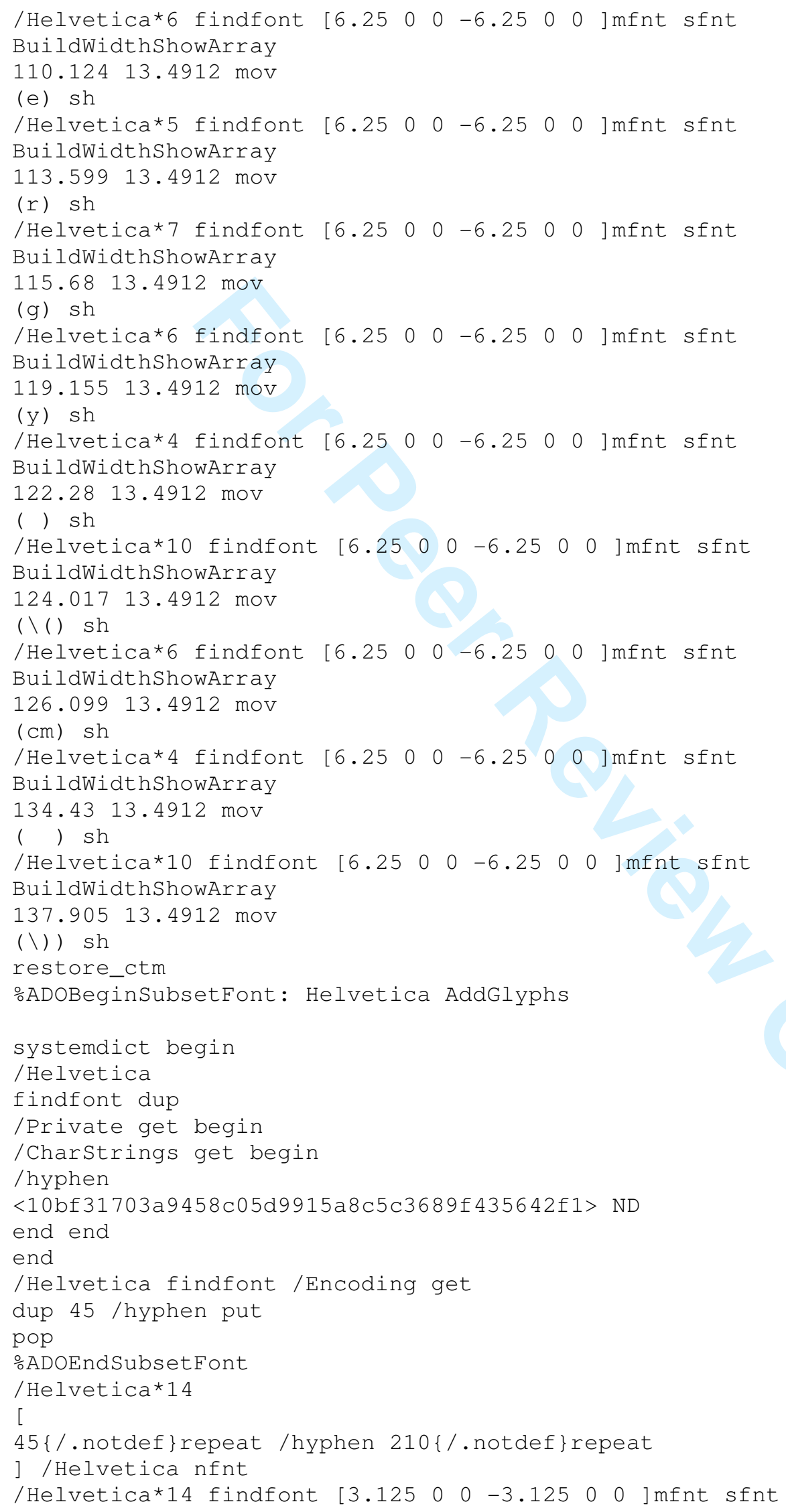


BuildWidthShowArray

$134.5819 .77393 \mathrm{mov}$

(-) sh

/Helvetica*1 findfont $\left[\begin{array}{lllllll}3.125 & 0 & 0 & -3.125 & 0 & 0\end{array}\right]$ mfnt sfnt

BuildWidthShowArray

$135.6219 .77393 \mathrm{mov}$

(1) $\mathrm{sh}$

grestore

grestore

Adobe_AGM_Core/AGMCORE_save get restore

응 PageTrailer

Adobe_CoolType_Core/page_trailer get exec

Adobe_AGM_Core/page_trailer get exec

은? Trailer

Adobe_CoolType_Core/doc_trailer get exec

Adobe_AGM_Core/doc_trailer get exec

$\because \circ \mathrm{EOF}$

gsave userdict /annotatepage 2 copy known \{get exec\}\{pop pop\} ifelse grestore showpage

AI9_PrintingDataEnd

userdict /AI9_read_buffer 256 string put

userdict begin

/ai9_skip_data

\{

mark

\{

currentfile AI9_read_buffer \{readline \} stopped

30

31

32

33

34

35

36

37

38

39

40

41

AI9_PrivateDataBegin

\%!PS-Adobe-3.0 EPSF-3.0

\%reator: Adobe Illustrator (R) 9.0

\%AI8_CreatorVersion: 9.0

으아: (ni van) (lac)

응itle: (BBRspectrum.eps)

응 CreationDate: 2/14/2007 3:51 PM

AI9_Datastream

URL: http://mc.manuscriptcentral.com/tandf/tmph 


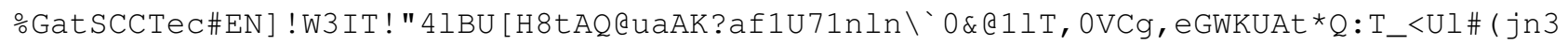
MRA3^" 9 ) 5RIXAMDe; De

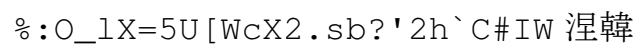

\&ZcYbuCako>_Vt\&pHTDQh5QAlE ( [_sa \V! [q.VNU^HpoNPQpq : JC>XCr?G?BolW7r9

H1Q6`?G (U_jqeQCHr8Y5If02K?bLj11 [M-*Hop1KDtW/b^A-

jVY?sC6rP.MO>ii\#) ??<,R4FGC\&hi,\#TJ (n>\$joh>J\) TpTe) 1 . :

I OcIaLW (qHYM] 6 \$gV8491H@fPlTbFBnT21@pp\&.NGO_0 鐗. L 'g@t5r-

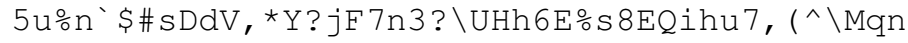

ㄷK\$S\#?' $\left.N C f G^{\wedge} O S \_0, \$ H=Y ? I\right] Y h ` e-6 h-$

WkMMu2Zr>hgtRY $\square$ 겧 \igW, \a2j<j?2U2XSaB5) 040 [ ID J'_G2; 'HrV\$9e5<k_q

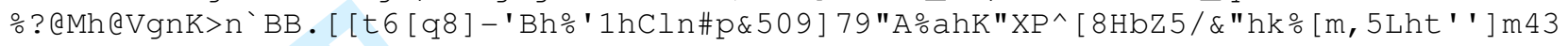

尸: $Q^{\wedge} r p e>5^{\wedge} W 4 g l m p C>9$

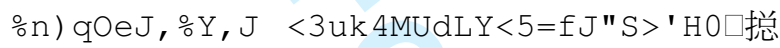

'DUH \YD [K>Im: [DqF, fomQRk@ $\mathrm{C}^{\wedge}<;$ FnF $\left.\$ 1 P 5 C J d \backslash \backslash N\right]-f e m j-\backslash F^{\star} r B \$$

K30:QT9"L\%`P_0DmQQ09s37`srU@dIc7p@fq/9WTrSmX\&@kp84_YU0 [ qW>bArQd-

hlOimCNr'E; ql5 $\backslash \mathrm{n}: \operatorname{Tn} \& 2 \mathrm{~s} 7 \mathrm{M}:$ rCOImOGQ $\% \mathrm{~L}$

M; $Q q$ : ? ] MTVCAQV2JR:P: h<nBG\&7=8hH0rFct<j<), X07X\#e?`pF^a\$Vh0L, QuU5Erid`; @a^/3//9Db $\mathrm{nFFbC9-KTUSmN43[.8}$

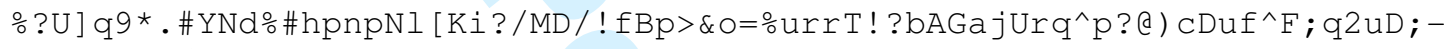

dQ] I' $\mathrm{cf} 7 / \mathrm{C} 6>$ tbAQ] JTT?XEHQHhEZQ

。UPJd.Q^IYCI, W\&F0-B*MnB: JGkPXK.a, .K9po! ; 'i-

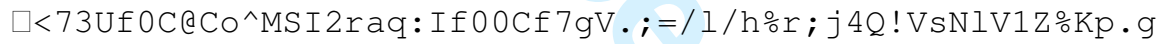

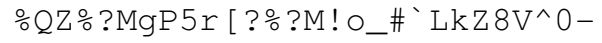

5Sgnc [HPO (LjIM'aV') \$Pi\#9Wl1hgTW:KX/RaR, @s, a 8"\#R\\$lmG=7PWu: LL9Eo@64LpXf:\$

ㅇ ? ) $6 \mathrm{u} / \mathrm{M}: \mathrm{crq}$, a $95 \mathrm{C} ;>4 \mathrm{rRK}(-$

IspNj55Y7"??, 5 i iMV2"1KC2?PCJ\&YnC. (e:S.PEs3B6Ra8IV3^A.\# \) XMJ\%j9<\$f3c8o\#tFs ]

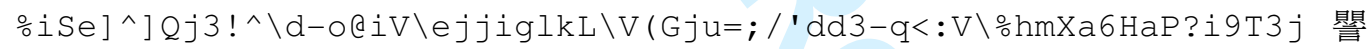

, CY6DgqZ_0; JR8q:kW $\square$ ) >Da 4

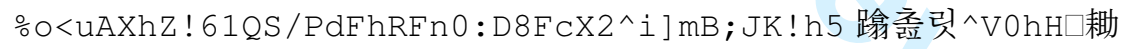

$\square\left(\mathrm{XHWSq} \$ \mathrm{~S}, 1 \& 0 \mathrm{I}\right.$ Srp] $\mathrm{C}^{\wedge} \mathrm{XS} \% \operatorname{VbIgFZ} 37 .=\mathrm{S}$

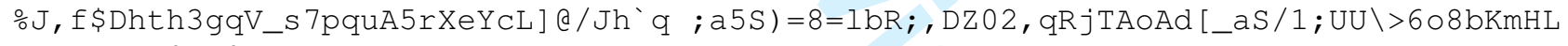
$\varepsilon=" I>4$ SR0Ab9H ] tWq'

? b"tl?\%)_CXXAH6,nG.s*q\$f\#n) 7XCE'hD`ng7luIseQ"f3`t\&rpj2s1nj\$;2>cha5G\#Y\$\%fsI! 1 qM' b1DEtIfD?kr8C:uEt-.,

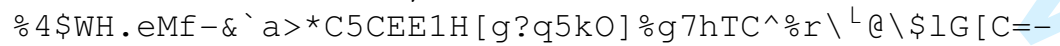

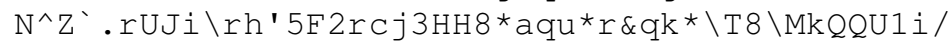

$\circ \mathrm{pUiZ}-$

$2\left(\$ . \backslash \&^{\star}\right]$, scLOV [aLe ( "IJ`Ae\%mQ\$GZc2kn<i^L (MH0bq! QUF ] AI\#mJKMWkgq02^. omQN (h9\?-

$; ! \wedge \%$.

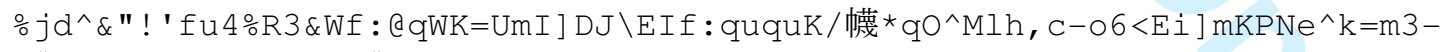

g\#JiLLh] q"\&^u61aW\#O, i ] MhgY=U

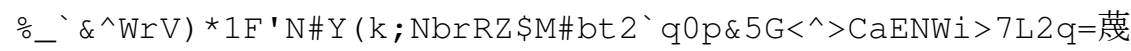

ea4q[tf1R] Y; Sb^Z31smsQ/X ( ( * *VdFI' [ :H1CUm

윽BjDg4e0h1'kH_s; UBNo] 4ABCV [HV: $63-$

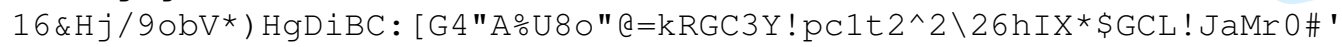

응 ! 4H.mq?ekt] o@NZsQ_XM) [ "\#ba4T7\%; tL\#sGbI^8c [ . ] VH'hKj2-

$\left.k I] \& i N S / f \# L q=T C Y 3>t j) L I b F \backslash Z r \% \_? \&-\right)$ DR

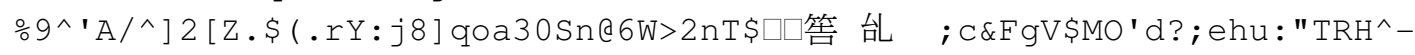

$/ \& '='$ of $\$$ fmb6t\&^GE2jo

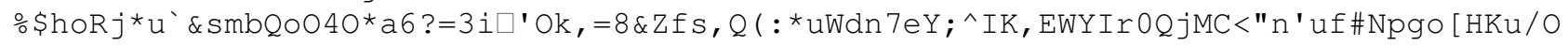
$\mathrm{Y} 8 \mathrm{C} 4 \mathrm{X}==\mathrm{ZqW}$ " $\circ \mathrm{C}$

\%LAPkZ5F3U (qMuHidJM\& [TNa (PCS; ] N*prmI3M4\%Wmdo`qf3\#jZYO>^\#qRN6pjCN5hSOX"<; 4N>Qn) BB J-a^E1V_e'ZfYAlTqd> [2

애hSp_eZfCmIa PkAQt2oQ<YtjSNk\&htlBpN/m; ; \$kV\$T3qR7\p_n] "fb@H) \#JZ'BBeYZ-

b $7 \mathrm{RHh}>\mathrm{Zu} / \circ=, \mathrm{P}^{\wedge} 2 \mathrm{qs} r \mathrm{~h}=$ Ot $\mathrm{s} \div \mathrm{Or}$

URL: http://mc.manuscriptcentral.com/tandf/tmph 
$\frac{\circ}{0}:>\operatorname{Xmndgl}[\mathrm{X}] \mathrm{D}^{\prime} \backslash \mathrm{J} " ; \mathrm{mb}<\mathrm{me}-$

dmGEe:508d6?f [m`J\%hBC.2f=5ks5g9trGDuR\&*r7; s69JE4T`] q>? ! YMQ $\frac{\circ}{2} \&-$

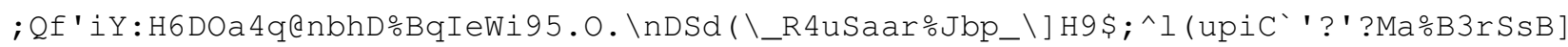
保政\&^Q"PL

HhZPkq/d-s \Js, 3bN:F`rIL; "7] 5a\#6e; Ql? ?\$\$ TaTo'A>TURe*j0m4I' \$WgQgYtQ'X*m3`n!tA5J 櫯\UYscflgmp。] D [

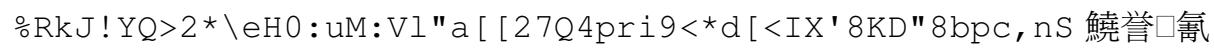
$\left[\mathrm{p} 4 \mathrm{~V} \& \mathrm{FRGn} 16 \mathrm{ZT} \backslash 3 \mathrm{HKr} 8 ; \mathrm{G} 8 \%=\mathrm{W} 32 \mathrm{~h} \mathrm{~h}^{\prime} \mathrm{OL}\right.$

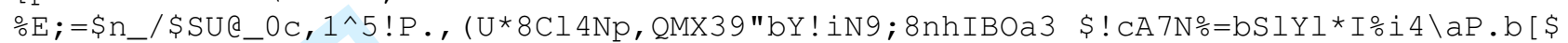
kArr] 4 YnY`H ['H]

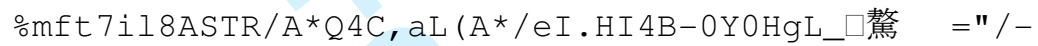
Il \#FHn 4qR) X66 [i?nVKTk2k@, YJ.\$?s-6e\&\$_P<*7Bm^* opouP' O.Wp9MrT) 4hn: 7@h\#mZ:/, .r82 [jo!n6Rh5?JkGG\&\$J\#<\%RQs! I ] C`rKr! 'NA"I` $0 \mathrm{cn}<\mathrm{d}^{\prime} \mathrm{og}$ ! (oa]. 'nS: qpHa0:U" 8 [8L

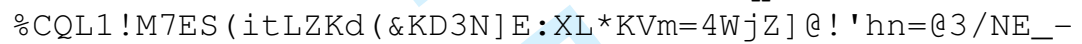
_hIhknh`oZ_YEr`S=Y/tC) 7CCi'U: 1qU) bon $\square$ ?uUGQ6 $<\mathrm{Z}$ \%?T_C\%B6[1ITA76>I.C\$[IsUeaq3UP_o2/(BL>t5c>7lcia1[I.\#?RLn7! iC1mg] 0c\%ta6>BHKd$\square$ ! "DSGVtsLfD<nfX!\&.G 이 ! -

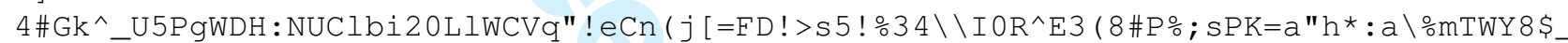
$\mathrm{kPp} \& " /=8) \mathrm{Sa} " \mathrm{pM} ! / \wedge$ 。<lXU3s; YA^jf; rhpoIHIaeduYkpA'r; \#<mlfS43YO9L:4rWf9,PVI*qCi/8h"`0\#qs`G_p/_^CF1ZMHBC920!ZX/@26rB\&dJ!a 。itoG>0) bh $1 \mathrm{~W} ; \mathrm{kh} 3 r O 7 \mathrm{KQ} / \mathrm{dQj} 9 \& \mathrm{~F} f \& B \&<\operatorname{PfF}(-$ , ZR, edIGsMmU\$ACPYU: qI3 4egR@nqB' \#5rQ"s9Y3oQU>*6<. ON6s1Q iNC ㅇrsoHuqeg ( \&-U/83T46n00ss, kT-

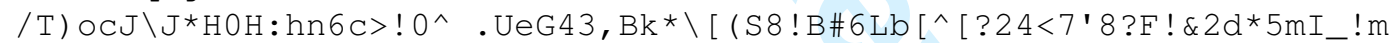
\% ic 2NfHNN=CHbR8kr9_Dr0]_a64t1RXRInh?i9n\$m) NIX\&, 4 \&\$`rZ) t\$HG6 'qR;MWk`5kPa3je38G;C?_NEjKerCdGU\&\&91p0g 8^

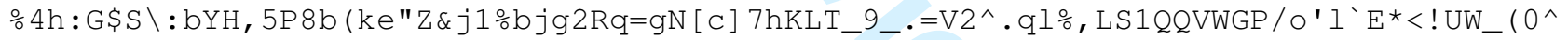
$\mathrm{kp}^{\wedge} \mathrm{qiF} 6 \mathrm{r}^{\prime}=\mathrm{e} ;$ TJHerE/eJ

○Ea. *7cCU) GjOm^DpGJgqS OP10glBFor8f3WT>*WV_QZ; kGQZBoh. IY9SCP/>YnU [MEeV=Z@:A\$igcq $[>2 \mathrm{ek} ; \mathrm{b} 0 \mathrm{f} @$ or $(\mathrm{O})=\mathrm{N}$ :

○QBLu^XJ; U⿺ [b-m/PrriNem*1Pj\#fYeB8Lp\#Pn02"KphA (ec) YKKehjZJSe $\square$ sor $\square \square \square$ 濽 $<$ EOV] Bp5Ae\&P9, Hpp!

$\frac{\circ}{0}><>/$ TO6KQ] UF gVV. 5\#E) V: Qc8\%, $\mathrm{B}^{\wedge} \mathrm{RXm}=\mathrm{QdCA}-$

\%LIE7\]1Sj,CJC`<6N\$HAlP^G(IC_O), a_KHk_<Cep?b0>AIVk \\%L4m0h?_g

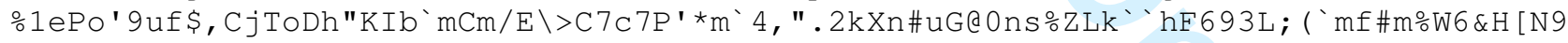
Knk; ‘kc (AFTS3SJU]

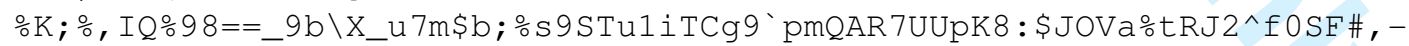
\$"UXX`Y [ $\mathrm{QT}$ I IG-D7S4"/ ( $\mathrm{i}<\mathrm{kPVPS} . \mathrm{m} ? \mathrm{i} \_\mathrm{b}$

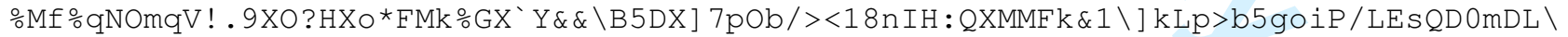
RLA=MkiuI5\#lGdaj?WIYC

○SpOipk-6H $\backslash[$ _rp97`DYg-

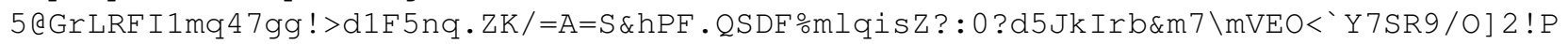

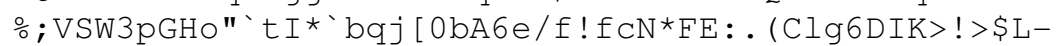

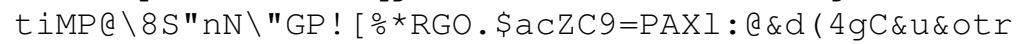
。GF \$) 3`22YF_Ud\%; R7m:nN^'>e! s?) OI.J=j*g'C (oB9<; S>3"T>Yj_F8..f9! /e00\#4*2510jXF34=b tN, Y:uXN-H5'_"] ehi [

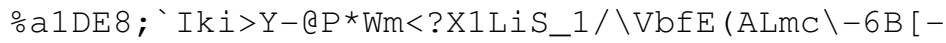
, b4CBUQE^Z ( $Q^{\prime} 5$ \$ I "HO9 :^XO_WI=0 [_ic>"/e3" [YAk1H_.bJ: [8en ㅇ) jH?pVD5p2iP?"! "\&nfLcONr8_j<D@ps-

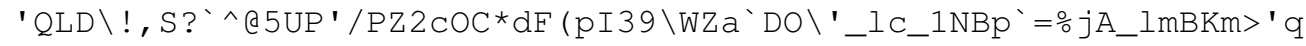


ㅇ.SSbQX ) ehFn:L6XY $\backslash 5 \mathrm{~B}>{ }^{\prime}(\square><\backslash \mathrm{Hq} * 2 \mathrm{~J}-$

o1Nk<ea (cI3ZSPm\&gGuEXK; 2n2F5Z [07` T) X \nm97b, A nnKuk2, a;.r] CYs <.

○04jK\&iDKN1A 冷 ( \$OK3] Q4-E0elEL\# [ ) CXW-

k3 ' DoYUqqaTtVMB \D" jAun>5 "5D?V9J; 3rj` J \>PUs3Z?XTdi" OUN?N, ' Goeqm

○) bfmEb_c4fO^S@ [ ] OM1mA $\$$ [Gp1H9"tQ6ch (\#UJpln^<kI, 9k;-BI_oDiOs-

$i Q \backslash ! \backslash j>g X J ` * V d] \# 9[E c \$ 3 c N$ ! LpN\$trp3gfq

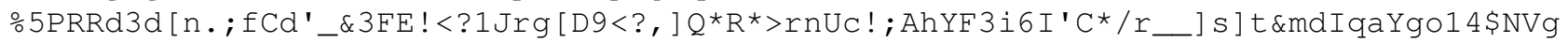
$>\mathrm{RRU}{ }^{\wedge} \wedge \mathrm{O} \# \mathrm{~B} 9 \mathrm{~N} 7 \mathrm{Wg} ; \mathrm{mWQ}$ ( GO

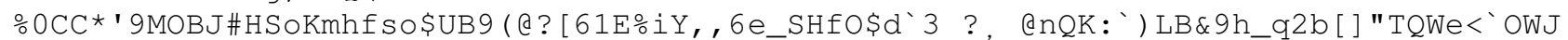
4_OG;7GnlEF

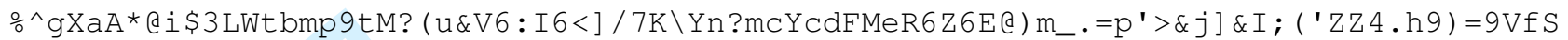
A0p_] $=` Z r^{`} e 6(Z, C " H L<C$

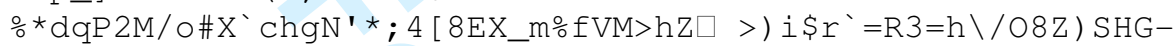

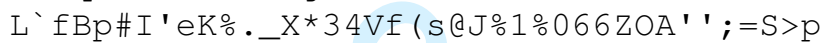

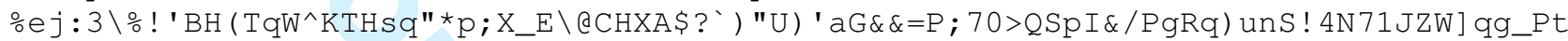
$\mathrm{X} 3 \mathrm{Y}] \mathrm{WGNGUA} \wedge \mathrm{Q} @ 5 \mathrm{sl} 1 \mathrm{t}] \mathrm{gU}$

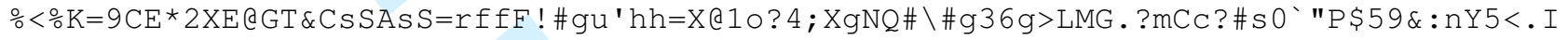
$\mathrm{YBdk} / \mathrm{R}<\mathrm{LC} \cdot \mathrm{m} \backslash \mathrm{X} 6 \mathrm{ai}: 8 \mathrm{jY}$

。\&.93Z, OACO-9FL4-: : \&MsV\#U2rGL,_Ws!m\CYMr!<U\#4/TBkA8I 4 *_

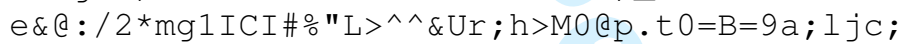

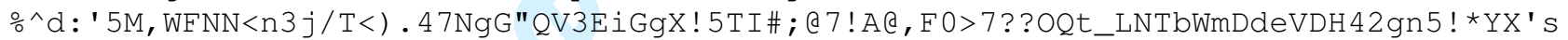
$r \$ \star W i\left[F=,\left(8 f \circ M\left[H Y^{`} 1 C j\right.\right.\right.$

1a8R8niI\&S (Bt*1BDc5s (1Jnd"9QgrXaPc1mK'r\(, 6m>A4mCk^31\&03: JtrO?LZBgUWjXN. ZpT\& .<_ D?09`aN'f:d(aP\*iE7>'

K100-U=b! 15A] g\$) 70S! Tce\&MrRB] iU"k\$FRWC_D-

$1\left[g @, j j E T \& g / Q K \$ 8 ! Y^{`}: 3 t .6 Z i T 6 h ! 6 . e J T \square: e^{\circ}-V P U 65 p^{\wedge} I Z(g p X 1\right.$

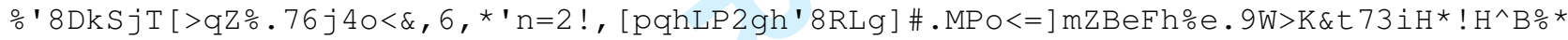
jp5IM.H'So52YC/; raq]

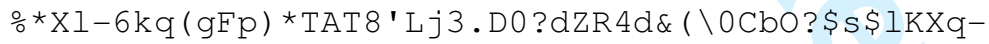

NabL=J98RT65\#loYgR'Kh\#rQ<?WiJ35BY\#Z@) i 9HB]P. 09DJcX? 'nq

○Pn:BE[ fNqMp.S"] @60>Md>K [rB37EJTGAB1FMIs@H>?aClelk3e,_cK! ไt?VGWBN=) Leq0J : CegH?P< $($ ogk^k4>7`WdfS5 7kK]rU

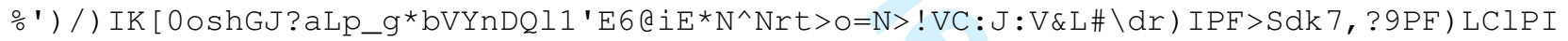

륓秾: *2EOL $\%$-*

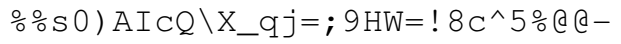

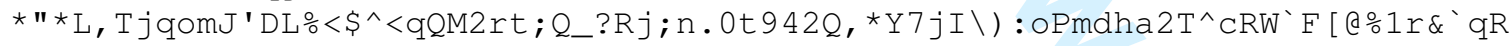

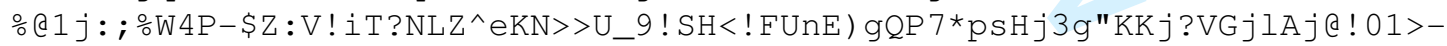

$Q^{\wedge} \mathrm{O}=\mathrm{q} !<\mathrm{sH}>$ WRIC $71 I^{\wedge} \mathrm{K} \% /$ prWtmoqts

$\frac{\circ}{0}=$ @ 'q/) "`6 (jed, AfZBlc-\#tu*`5-

uJ 'Pq@iuLhVU" ( : tM2iuFoHg@EX [A<q.<'] ) \&O]M0QOuM \JM? 4 "KuAcimYn66*/, t7E-

Y/ ! 79\$!2d3ai4"Dgi'/s\&/Z.9?hb*U"1Q) GM78I_An!4=(Elki"*>pbh\$M1OX_\$5m9\$\#u) "Z0lI'd51

$\mathrm{HW}$ ! aC=, U2H_9j!) s7KbK,

-of^ugQ8XdK] ำ (dZH67! Qh\#, .; ! 7KsTp] E6f26\#-

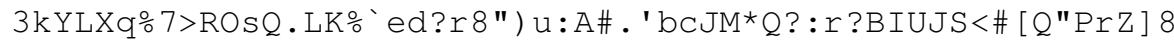

\%3"fnRRfEVJPZ\%QB] \#’]i/Y/isPtqj/\%) Cl!g]'4dQ-'Lu (6-

DFT \$eB2lipOAe; P\&e6\$ri*OfhmN\$B: I 40XKoA0uYgrk\#_Q\$kHU\$Q

k $\mathrm{kJ1R}>, \mathrm{R}<\mathrm{k} \& \mathrm{GS} \_\mathrm{ROL} \_\mathrm{W} ;[$ ] C:B\&A\$7aG" [T\#: [2dOT-

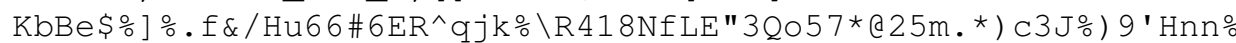

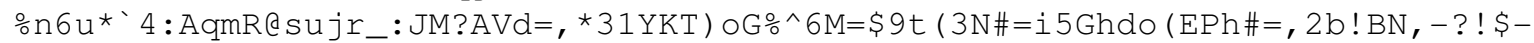

${ }^{*} \mathrm{M} 2</ 6$ (Qi.bRnpT5:q3P9,01] O/2

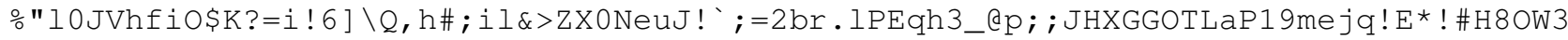
$\mathrm{nbAeI} 470 \mathrm{~F} 9 \mathrm{~d} ! \mathrm{mHS} \circ \mathrm{C}^{\wedge} \mathrm{Y} 4$

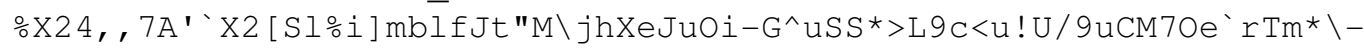

15310VQPPD) NggR9@, W') "; QVajfY" \$Lp8ud

응, s [Co9WILeP\$\#be, 1N@J ( QP \$Oi : kDmON : *OB. \&PtSatRH>DDdZmU (aF"CS.g45, FCo 退

, ebD" ‘rd/Noug] q9\#scW"?cY

URL: http://mc.manuscriptcentral.com/tandf/tmph 
4 S [i1 (SO9J\#6YOD"] 5<j<P'L! (K: OJALOhm`( oa@YVB_86:_L\&='_OWQD2> 9Pe\$?, 7W<\&=_q_Ei?e ! R68PnC0u7115C\#: $55 \mathrm{p}$

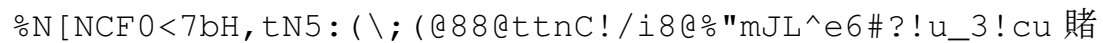

口_JULHiT^5UFQu"1Rs6\#!cg18s\&8]-fT`! "p<

。dR@5Y'C6!: ]: h<sVLe/; 8d.03pb!>(3K\%[:TF-

hiBpiAhY (\&JgDhKoabHn [:6)_Nf`d[dYq\&?Q\%riIj\%qWoQEl\&W5JmUse

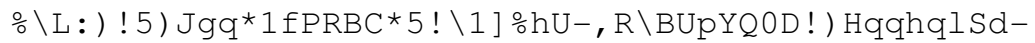

$>d ` m Z d \& h / \backslash K \& I B g E 2 \$ \& 9 N r:) J ;><C^{\star} J f, Z h C d 9 u N " G q a \# V P ` G V$

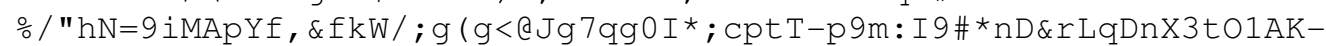

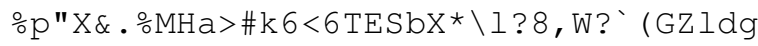

$\frac{\circ}{0}>\mathrm{io} \mathrm{PN}^{\wedge}-$

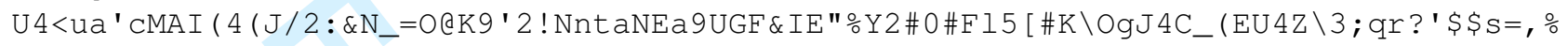
$6 \mathrm{nlWOk} \wedge \mathrm{IC}$

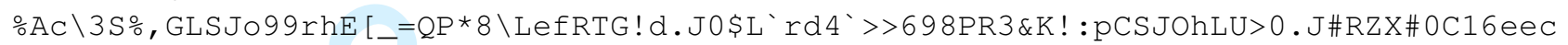
B9Ym! gJiaW! \&"\#P.>S

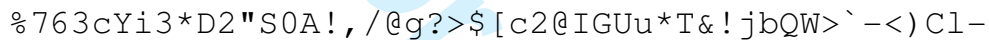

9TWpRQU $\square \$ \circ d<C O L>I \circ, 6[] R I$ eg\$bgfTn51"7_(ogX-FSNf1u/

`sOCOTI6m7CsZK5K^j\#<C ( (e\$PaLno` @D5 \( luLEJMHu\$NRoMK\#!a`65\#^RN-[r7TUJs-

LKn'h (@8n-\tT5q(6,. =Fkm-e, ..e-

\%/ 'D_JAKIqA`EK. h>h:CV\$.SFArDKYrSY-2WA>6TDNJ=`5DB@PH`ZJM] j/eD<im-

!ee', 6k,V4J1^DXi) alsF3to_KI9)! \$="Y

$\therefore 8 * \mathrm{D} .\left[>\mathrm{VmWXOu}=\mathrm{MqC} \backslash \mathrm{sb}, \mathrm{L}: 21 \mathrm{p} / \# 8=\mathrm{F} \# * W 0 \_\mathrm{US} \&>\mathrm{UF} Q R n c g^{\wedge} 1-\right.$

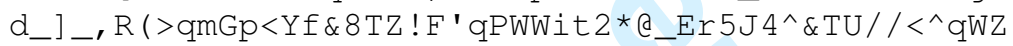

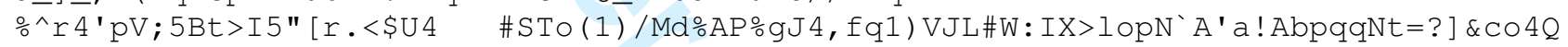
ej@1?_-91Q70\t\&

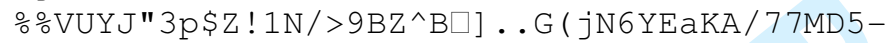

^Qj2V. @16JeZn6\&r?k\#XSD2u0BQ3M; l?) * 'aJ@VhFVW<mb (-1@`WXB/B

○H\&G"6_^QbQHtGfPS :Y", Y, k 7D-C*Gk, i Tqi\&L"CC?M<BigC?G5\&hns*e-O/e\&, -

( [RBN.Mp6> [I:_\Hu6FERqi4UZ! uP`\#N<BDL

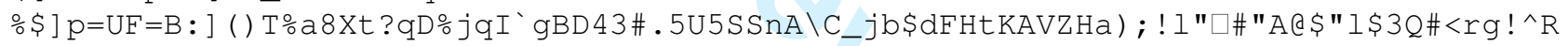
$\mathrm{Em} 2 \mathrm{M} \div \mathrm{XV}$ ! $\mathrm{YZg}>$

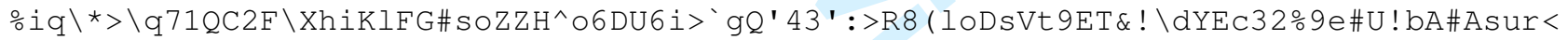
$1 \mathrm{M} \#{ }^{\prime}<\mathrm{n} 7 ; 5 \mathrm{ankCP} ;$;

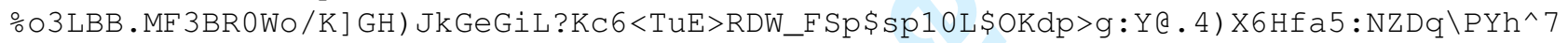
N! iiipG;ON`\#_sAg-4rGP

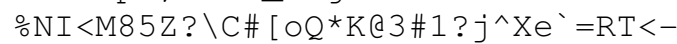

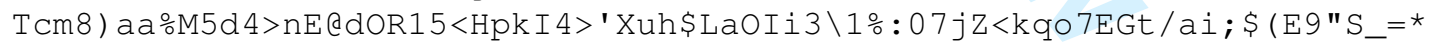

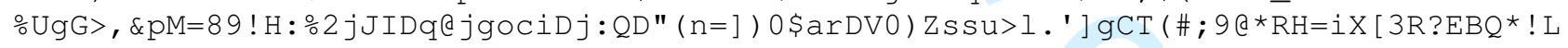
) : / 7\$7>4XK11'*^X`DQ

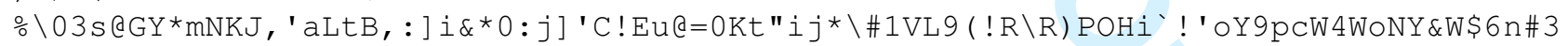
$l * \mathrm{pSfEBK} \backslash 3 / \mathrm{JAlEYepD}$

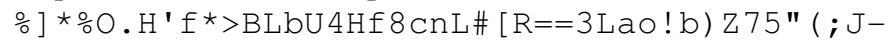

' [ jh\&EFUKPjijKiYM"sY2KG_AP<R=9`3e"_H4KXEV1e] eq! "m?rk:G9oal

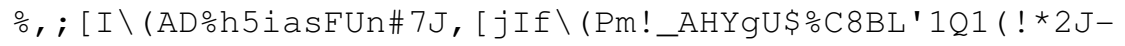

B_>UI!B 7b \huE5[MuG\$rETh/4] jjOXJChX:h5U1YZSE?g3p

$\circ \mathrm{E} 2$ 隇

^fp:CWpLeri\& (WWAgPi9u, / . Q; NXbThp, 8 (X'17! XU' *9=BaA3 ' aEXR3H\&W7L ( ) sORR\&G '^UNu5\& ] QF 4 \#KR1>j_tfB

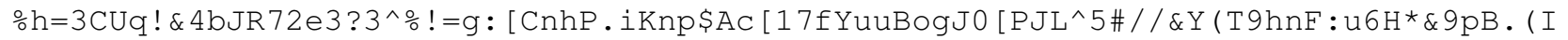

" $\mathrm{Y}<\mathrm{G}[\mathrm{\text {" }}$ [?Rq1 晨 $<) \mathrm{Wrk}$

3, 9=@rAO\$\#dfg'kk"d958) "WT, VH [mf_. \Y]D'KJ"J6] sG`FCpPd"-

9 ! L\$Kt 4 \$O'_A 5 eA?) X7riMBkqaEG1K! - "fZo\$r; ) 6

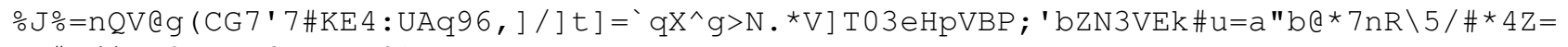
L (\#gjiP] 2XYoa6stY-NO\%

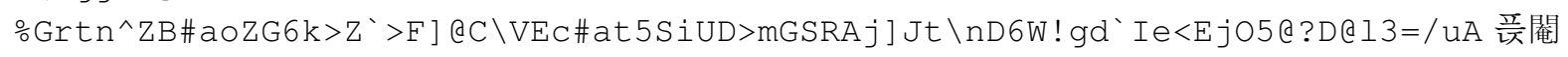

$\square(>\mathrm{H} 3 \mathrm{LO}) \mathrm{L}$ ! \$C\&Oi\%; SG5 
\%JuVoa: (JWOI^X:a0>e8" 羿'EQ94QN`d8>^CIQLC: -

k@ut\#bkCC@\&/i $6<{ }^{\prime} \mathrm{GjYaY} 2, \mathrm{o} 1 \mathrm{iBR} \backslash \mathrm{n} 13 / \mathrm{P}={ }^{\wedge} \mathrm{C} . \mathrm{R} 8 \mathrm{C} 0 \mathrm{I} \backslash ; \mathrm{kL}: \mathrm{f} 7=$ "<_1W

ㄱtOu'fm/2 $\backslash \mathrm{W}<\mathrm{Rn}(\mathrm{RO}-$

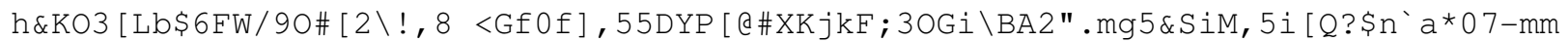

으 ‘ $\mathrm{j}[\mathrm{ZO}=\mathrm{TXmaZ} / \mathrm{gpk}$ ?pd=uaM/OqbQ] ViOch9X4_e] (1 ccaq0LANe1-

:Zh5!kiO*BJ. (eSPj2k"*)\$'; "T3eTjZhA5^r $>\mathrm{N}$

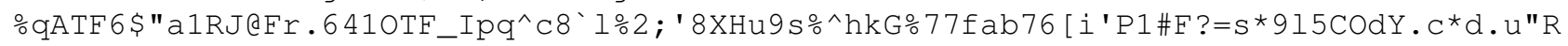
hK' $\mathrm{qEX}>\mathrm{Et} 0 \mathrm{~S} \div 45 \mathrm{U} 2 \mathrm{r}<\$>\mathrm{S}$

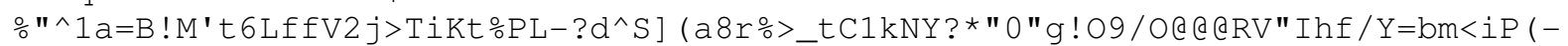

$\star />" 343 \mathrm{EPM} \$ \mathrm{VQ}\left(=7^{\wedge} \mathrm{UE}<\_3 \mathrm{BqQ}\right.$

○GJWC : \&C/:SamHUm\&kmPLmbC<?J [hsiqdim3FGK [L79d_GLqXhZAT3; a 8) g*;m) \ZA*" (QArtgE_@6eK $>r-Z O, 2 \mathrm{EV} 3 \mathrm{~F} 2<=\mathrm{BXIeTm} \mathrm{Q}$

F J\# ( Lf' ' , oDN2*'>! ( @Gr-HDs>LpXDMuHK2V1U, bOp: I6 'N! Ya3*ie>^q*HFB; 'B-

C3T10HIn9UUPFa9S3DC0 4bm94f<d6_qP

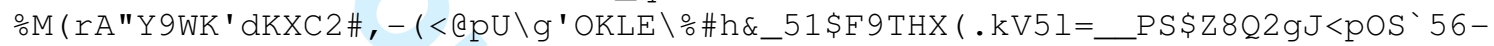

foDn; [ "mRc18, [\#*faG\#, QGMT, 8A*

RMjgLkQMHh $\backslash: \mathrm{d} \backslash \mathrm{f}^{\prime} \mathrm{Q}>\mathrm{\&}^{\prime} 5>^{*}>$, TU7N5Xk!n] VLt=Ahh-DMjkP\%11n1:2ZCsXgr?d009Qal-

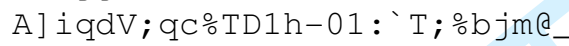

$\circ \mathrm{Q} 8 \mathrm{de} 8 \mathrm{E} 0 \div \mathrm{NF} " \mathrm{~g}] \mathrm{Xh} \$-$

$37 i$ ), H ( , OVQUM ' , _T1NGEt $8 \mathrm{~g}$ ? GONG<, a@ ] USH/XJTdD; r ] 0CJEuF5_hA` b=<2@G! PpCeFhHJC29V"Pfq Q 19

D ] =A9, ] DSC=bs\$' \#; e*J9Z6 (pAe 餘=urR11ZIn-LSNT4_A`N"I'k@Q5nब; 9h6Zu6<-iu4^I6Nn-

OW. O*"e.YC"QYSerRE

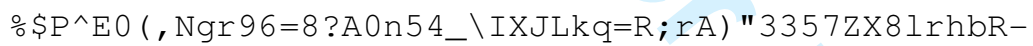

$\mathrm{B}:(\mathrm{mt} 1 \mathrm{~g}:$ \$G7QU17h\%a] O/<nK8cs"Sj:s`\%-A, i-GJD\#dLq-DUoC [

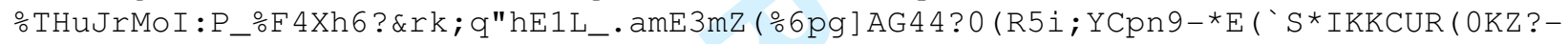
bfC'F3f@K) ! : 0OGSCPQjso:

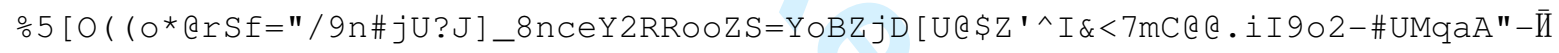

! $e^{\wedge} \mathrm{hNaX}: Q 1 \mathrm{q} \%-,=\mathrm{a} " \mathrm{I} 3 \mathrm{dh} 6$

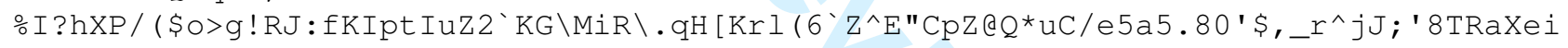
$\$[\# ;$ Oar ! MF (* LIBQm

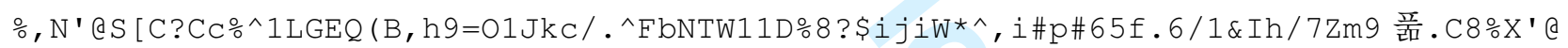
$\mathrm{PE} .<] \mathrm{pS} \mathrm{S}^{\prime} \& 7 \mathrm{E}=\mathrm{Q} \cdot(\mathrm{Eg} 7 \mathrm{Q}$

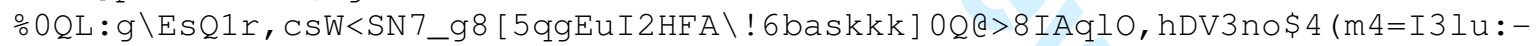

7 J\#') $\backslash \frac{\circ}{0} \& h^{\prime}>$ ! $>$ NUhP [ / ; d ] \#P"K

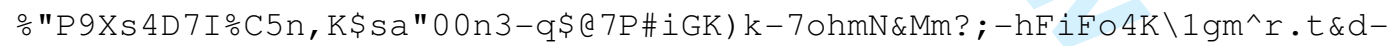

$\mathrm{P}^{\prime} \mathrm{NIe}$ II] sJIIiRk\%qT'-PdFu^U]-\%[Za

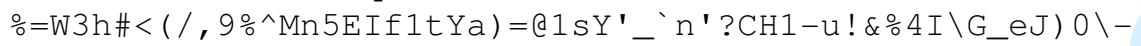

$j W: 75 d B^{\wedge}\left[Q^{\prime} m V \_h \$ ! A J K M N\right] F \_D \_R, ! \bar{C} \div\left[u V^{*} h 40 @ \& N 6 u\right)$

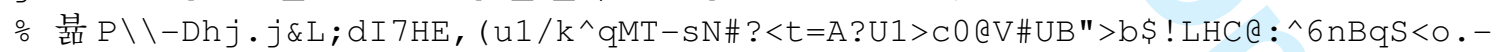

WBV? jpfC'HIMWRp (\#! bS19

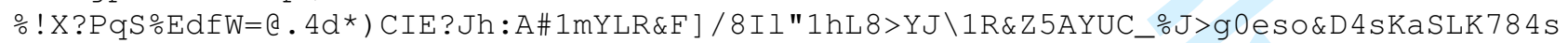
$4 \mathrm{p} 4 \mathrm{XGP}-\mathrm{Q} \cdot \mathrm{qY} \cdot \backslash ! \mathrm{S}^{\prime} \mathrm{M}(<\mathrm{h} 2$

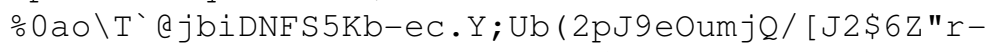

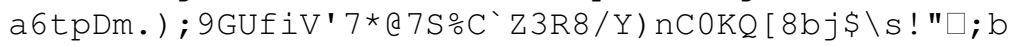

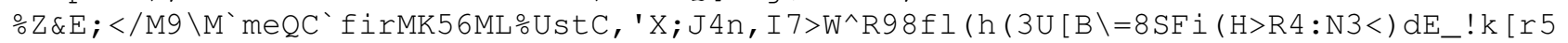
$\mathrm{M}>\mathrm{udB} ;(\mathrm{s} . /$ ECC (jD2'YqQ

\% (Bfb\#ZtM\&rjo\&?Wid, eFE<dPjqc19n*UGq5! ?D_:MSj5VM (UoV) NJ\$-

[SCer?' / HhZPi \/aNPr3Y,Adl [H'5*"<7Ra_<, oedPZ; i

。kMpQLR<'WU\$ j"=] 0^98ji@NJm_s\$I' . 7tcLS897Ymf: IJ [ a jC?qUbuQo/625g=i \$EaL6UA=b67\$XXr"SMI *YE>LQ\%euKR! 4 ? j\$

○QiZP\#! ?f?VbS ] 0 . \ekneSE1S ! qC9HW-

E`iGCEW_N] ] \& : 'M@2pN.jQ"k [jO/ !>(^\$:X\&hJpG\%l(V) ] jVrCos/ZG^pN"@99@Z^R48:

○0 dW: 6 'MoU7/Vlbbrc ] HOAIA?KVC7A [K, $2 ; 0-$

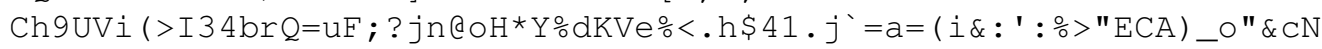

URL: http://mc.manuscriptcentral.com/tandf/tmph 


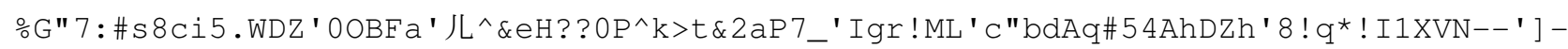
aGIRfTN $>[p=g 9$ lot

○gRsFnGrsRZ\&7E`6EFV0Y8IUDJ, Y\%Hq; d03:bdT:` I38B5VtBK (Q') U) _s, _! bTrEYVDZp2) <8;^d@5G $6 \mathrm{PO}->\mathrm{AnVN}^{\wedge} \$ g h q e \mathrm{Nj} 14 \backslash$

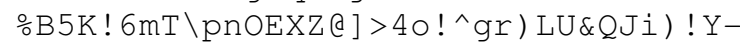

.5K"KX6Q_' O] CVA<`7\&S> (V4\&\#A9FCC? 4 "Hp9! \$CQi3QgN`! 3 (E`AIur! GHPV' OU^1

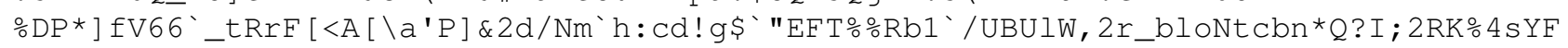
rOHK.r: 1 ?L $>\& \mathrm{C}[; \mathrm{B}$

유) $\circ \&<2 \star * \circ 2 ! \mathrm{VT}(>! r q) \mathrm{E} 2 \_>>$. 鲟

$\square /$ YR\#k`I.^X3tmZ; ; \2um; j\$H" ."G9RHKCW(UJ\&7\qL (pM1L (hjmf2`_86.Ek5e 7j'!

$\circ \mathrm{M}-\mathrm{p}<\mathrm{m}(\mathrm{Z}>8 \mathrm{HjUsX}) \_\mathrm{R}=$ ' Q6 f InsQKEbAt -

VU`ri84j_K"UPpi@c10JI,^nmjH1\%n\&Ag"1q<s/Km*r[Ih\&AKZU>9EVB>n9t^aP $\backslash>$;

\%/ ' ; iurm[Z9c: \$W. VpuOlSnC $\backslash$ ) IJdJ-' (D21nC?PDFWQE5YIQ-

ZLZhOO"BIEYe\$6\*IoTp17i1Y"8<[\&B! @tDe_MqRg5 (^<PSIk;

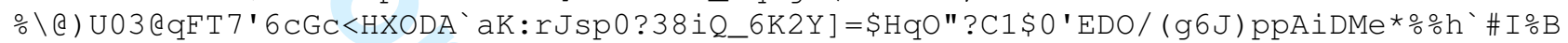
ㄴ) G] Aj] SHE710\$Q \#mY5

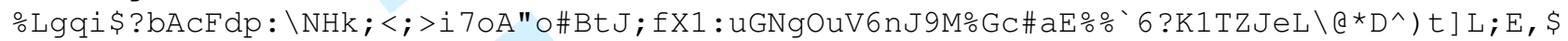
D) - mYM3 $\backslash-C S \#$ ' $r \backslash T 96>M$

F 2sNCbN@J$QpL\&VQICp`<GCJC (jfJ\$!W, K*" "Sj^M1Z7LEi/A4J`_gG! -

ABXI.R(T.KZt'sC.6ZYt]MX' (15EXN\&IA56IAQ9t9_

ㅇ) iU-ki+G>MR [o_<q"N\; ar^/tG6Y"HlJd-

Ai $59 \& \mathrm{~m} @ 9 \mathrm{E} 1 \mathrm{~b} \backslash \mathrm{t}) 0: 9 \mathrm{q} / \& \mathrm{COgHK}>0 * 5 \mathrm{iH} 1 \mathrm{pB}<;^{\prime}, \mathrm{h} 2 \mathrm{~A}^{\wedge} \mathrm{pfA} 31 \mathrm{I} \% 23 \mathrm{ZWeF} " \mathrm{rM} \mathrm{M}^{\wedge} \mathrm{E} 6 \mathrm{i}$

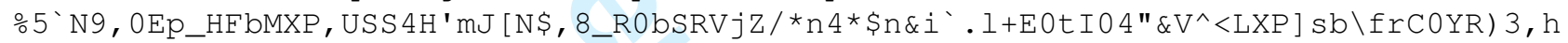
$\mathrm{b} 0 \mathrm{~g} / \mathrm{hpF} \mathrm{eq} / \mathrm{mXH}$ ! Ntke

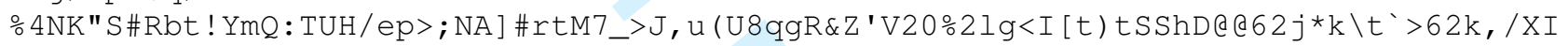
$\mathrm{IRB} \# \mathrm{KQ}<\mathrm{k}$; $\mathrm{YF} 9$ ? $<\mathrm{pba}$ ] SS

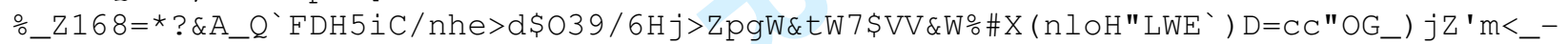
$/ 2<\mathrm{QK} / \mathrm{q} "<-\mathrm{HpG}<[\mathrm{LM} ; 8$

。GDjD! - \#OQ/JK^X4I^[2:/9! \\&dd3\&=1K6*> (i19'Xt74"X_gYtE^E`u6fQ_.5p[9_3iq-

u $9 \mathrm{dH} \$ V^{\prime}(0 \#$ jObks ] a $5 \times B j-p V r f \circ M m M<$

ㅇU#i.\&( ' O>] S [ ) Dd>iQqi=h]_l] hJK?2,P9] (N-k/o<*6EFH! ?c67Fpmd!MLeKW7WKr) b 掴 $\backslash=6 * d J K P \backslash G e E,[] ' g 2 u \# D X D T K g$

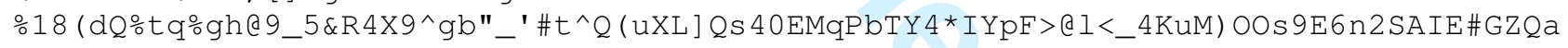

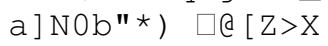

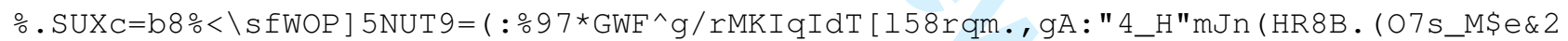
$\$ 0 \$ 8 \mathrm{n} \cdot \mathrm{u}[\mathrm{r} \& \mathrm{CAct} 49 \mathrm{sjH}$

\%6pM2a0080EkL.>1/dpk9Wd2q, nr\%Hp (712V- (\$Rk 捿; U_5iqZ8hK3 'B' -

CC, iC/\$961N\$di*5tq@e3QT>64iGm1tP\$Akak\$

$\circ \mathrm{b}=\mathrm{HK} 6={ }^{\wedge} \mathrm{Ce}-$

fM' 3c6U8:900KL. ihd (c ! cejncst\&; "QokI) ZqeVpmA!m\#q\# IngC\#.EZOE2 [A8! ZIN2d/N98@Sqe6C^ $1 \mathrm{CHE} 7 \mathrm{gYb}) "$

$\left.\circ *=\mathrm{N}={ }_{-}-\mathrm{SUU}^{\circ} \circ \mathrm{g}\right] 6 \mathrm{i}$ hut $:=\wedge 1 \mathrm{ZL} * 7 \mathrm{~K}^{\wedge} \mathrm{RBPS} 86-\$\left[\mathrm{~b}^{\wedge} 0 \& . \mathrm{Y} 6 \mathrm{~V}(\mathrm{C} \% \mathrm{q} \% 1 \mathrm{~K} \vee 8 \mathrm{~V}-\right.$

$\$ G \& S Q 3 L S$ ' OXA? <Cp0HViZgPAGCre* $\mathrm{3} \$ \mathrm{~kW}=\mathrm{F}$

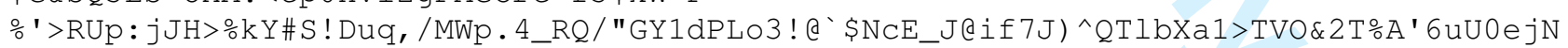
$\mathrm{AJ}]<\mathrm{RZ}>\mathrm{hi}>\mathrm{gif}^{\prime}$ ! $\circ 1<\mathrm{V} 3 \mathrm{~b}$

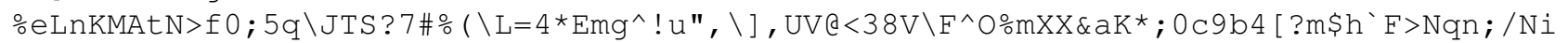
$\mathrm{K}\left[\mathrm{ch}<\mathrm{J} \cdot 10.4 \mathrm{~b} \div\left[3 \mathrm{VE}<\mathrm{N}^{\prime}\right]\right.$

$\left.\frac{\circ}{0}>\mathrm{jt}\right) \mathrm{aS}, 3 \mathrm{~B} \& 2 \backslash \mathrm{G} 4 \mathrm{P} 3 \mathrm{QC}-$

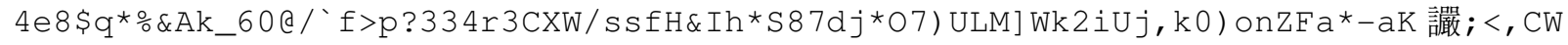

ㅇ) R3AjRF\&StQC"78?. Li\&`PP`uBA-V [ 8WZRQ [uf^5<QqO=03C 75N=-

$\mathrm{n}[<3 \&$ ! 8WZ6IB; , g/ ; F9unV@ ] ], CKF ] eK\$\&\$=\$O5J]U [ ! 7s

○OUFR1 \8e11@rcEr2lXcm, Xqs07Le; 5\$4>B; d5W_hicj/e>) C^, 1MCoJ [mD: j*@nl3'H-eUDfAjOYF ] F JN9b: $\mathrm{k} ;<$ ?०VmW2 [ ! $\div 5>$.

Ft\#8SP_mYCgFFLN7CC (.2'^] fSV [HYCS<g3i2sWk-F>.e.Sb\&'CPIYm\2=;Rs34g01dL3>(ujC?\%\&bI'GB2Q8NafNfl<h:qBG5> 
ㄱFPM $\left.\left.\left.\backslash 0 \backslash d / S^{`} V m\right)-/^{\prime} q 5 \_7^{\wedge} Q \_" \backslash \$ \backslash\right) a\right] k s @ ! t J `, \operatorname{mMJF3}:$ 秵 _S"' $1 \mathrm{KjK}(\mathrm{GD}$ \# \#TD8H (mRpZ> \I ?> '] M_< <VN' ( [ ] 2 ?>J-X

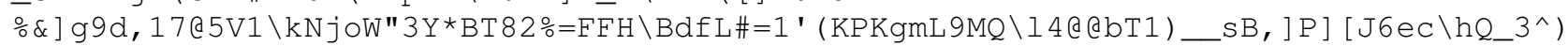
] i>aAjHWf9p_L (G\%d'-d2

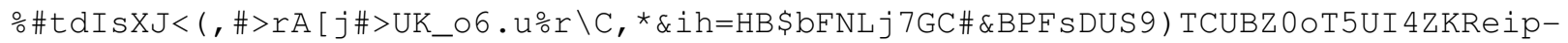
$a<j] \backslash A @ r c 27 \mathrm{KL})$ fO) J-Vi"

, aXm6\$>aBnYJL41XYRm\&81 [ IM6Y 緑

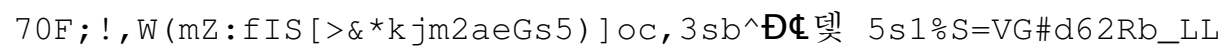

N: 2Ei ( ) /Ad"rnYLS [bq5L (RABG6>GL/F "XHogElQE=GDa_e" ! [ **@YB\#RmOA; [Fndi (D (G@rY/A! Eb\& P"CfopDI\$1q20\$7N>

-I>QSHRO2NL7g 枺: >IXN [*\#=De33SK3?g38Ntbe_\#fh) \ID [\#@uUl 苂

$\$ \mathrm{k}\left(36^{`} \mathrm{q} 1 \mathrm{~s} 0 /\right.$ ! , M/^KrNAk0e/a*, <6e: ) R \jjK7o

ㅇ /)n (50tVl:_">Flj-@MUHPd5b14s<G" [5j\%WCd)-

$\mathrm{u}: \mathrm{HL}=\mathrm{qu}[\wedge$ aMSK8BiU\&@C*Lf!TE $), \operatorname{VrKP} 0 \mathrm{e} 01<{ }^{*} \mathrm{~A} 6 \mathrm{Yp} \backslash ; \mathrm{X}(\& g-\mathrm{B}=. \mathrm{ku}] \mathrm{qT}$

。UunbeGqf8Z: [T (Vg. \LlkXdqQ) (oRdE>`Y_*eu2) eehoV\#2 . Wg j-

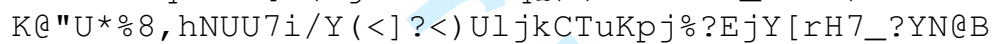

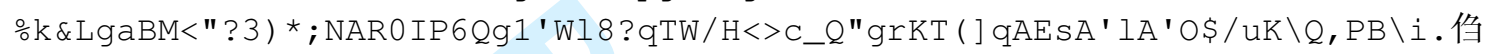

@Ba8mL\#; <=^5I\$.n/jJRF.H:Ij; I

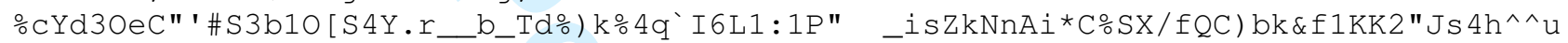
YEj"h<t*O, rQ.

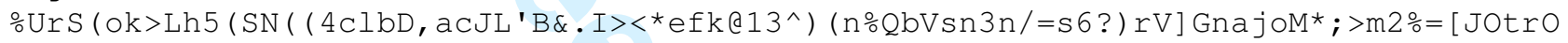
C_, ITSNj["KZ] T*p6E_9;

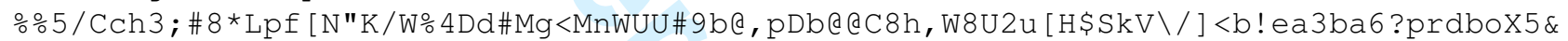
] "GCjEAjCZA4S7 (H'TI

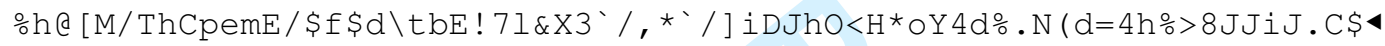

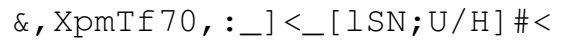

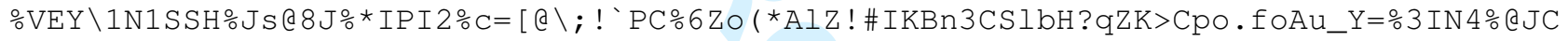
$\mathrm{V}^{\prime} \mathrm{W} \backslash\left[\mathrm{C} \mathrm{aE} ; 5\left({ }^{\prime} 2 * \mathrm{~L}=\$ 5 \mathrm{~F}\right.\right.$ !

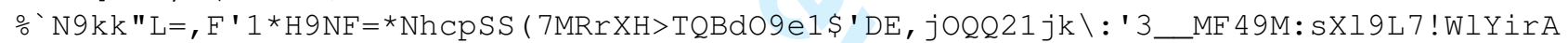
$\mathrm{g}=Q \operatorname{Tm} \mathrm{T} \backslash<\$=\mathrm{knbQsGQV0d}$

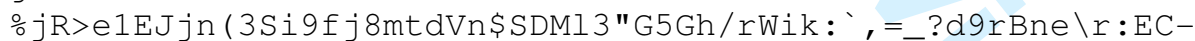

2*233' (Y36ES\$; Rt<? 9YSfnT? 4h, [\$*. ( ?*! QU=YXP

。EVI2BMfm^_gSrJMVs\$h?0SF ; j"99M [t! 4M_3M2_GAJD (1k/t) FX?i29gdkD'gr] '\&C6d! NVqk [\#hTp "@RD\#3A”'ghls_ced.

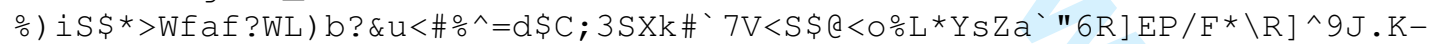

Vr! 210>kq_47W[hY5\#4E\#;Yb6nT8

。dkF2/2, :JH]) (URo>\$e,P, >e! =i. ZoYqoNL 7LXQLd^C; 6p [HW", ) L8iq1is"\$1 [_dDcuj0D6W?Tu> [) $1-\mathrm{Y}:$ flodcomHon ' $\mathrm{N}$; ah

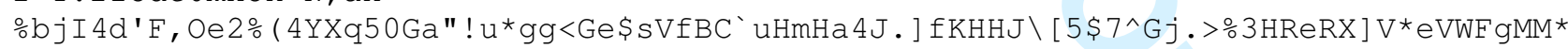
$i \backslash \mathrm{Ul} 4 \mathrm{AA}: 8 \mathrm{E} @ \# @ \backslash \mathrm{m}] \mathrm{m} \$ " \mathrm{dK}$

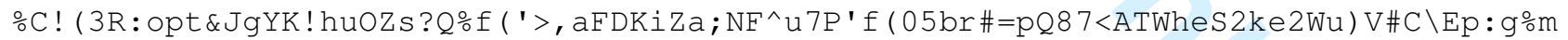
$\mathrm{k} / \mathrm{V}^{\prime} \mathrm{pHY} \mathrm{q}^{*} \mathrm{O} / \mathrm{OU} \mathrm{Uigq]S} \mathrm{S}^{\wedge}>$

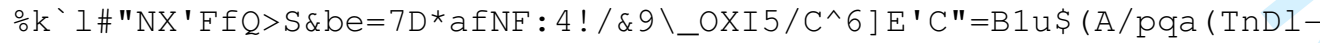

@ > O>04Xtet \$cuCAf [nN ( i $4 \mathrm{dWi}\left[\mathrm{H}^{\wedge}\right.$ ! K: $\mathrm{f} 40$ ! 2 "O

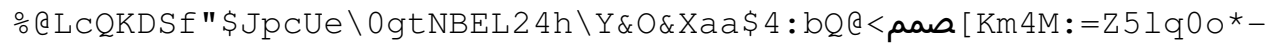

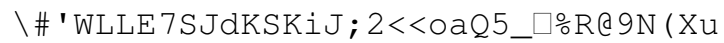

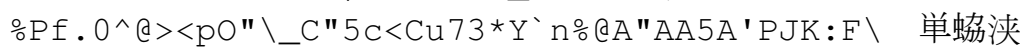

콤 $\square$ ( ?a (J@^aYqE\&9XM5p_\#; 0 \$ 'Pr`WhI30`HEu0khYJ

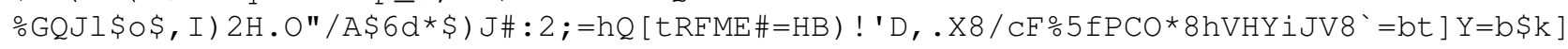
$\mathrm{MZO}<\$ \mathrm{~S}] 7=\mathrm{kL} \$ \mathrm{~A}<, \mathrm{B} ! \mathrm{IV}-($

a (PI [T930Yi-f2Y<K0^\&JTpR2D \2OsPq=W"YP\&\&"Yij9F6qeK0-

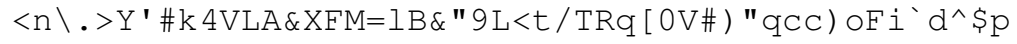

。m5Ag 4 *WjKR [ D^7\#Z`U8t] M7^<9R; 9DB/fa\#F !W7S\#nF9T/29L) TMs12f\$`q1dEn2k`PnkkKSD: :JC)( sV\&69U11;NN_CP)*gGZ"_ 


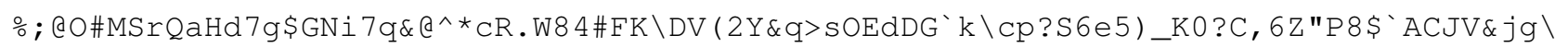
$\mathrm{U}\left(\mathrm{V}^{\prime} \mathrm{QR} 3 \mathrm{~B} " \mathrm{fC}<\mathrm{bu} 9,\right)$ ! Pr.

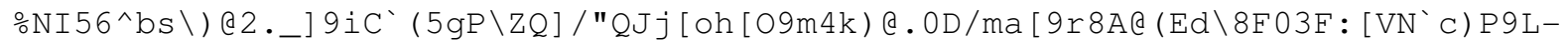
) (d*i`7OVCANI $\backslash$ e $/ \mathrm{En}] \mathrm{L}<\mathrm{g} 3$

ㄴㄷKaoQqP=Lf6TfQ' jo`njpKgW1TBY\#ID, L*Xo2eIh) n: iBoPK:mHtTsMh\#W, -Bl-

$\mathrm{EPXa}: \mathrm{F} / \mathrm{n} 0>2 \mathrm{r} \backslash \mathrm{S}$. 'C2QFZ\#) BU0spU1h/76e

。WI\#Mc6rP\$UJ\$: : 1J) \&1-

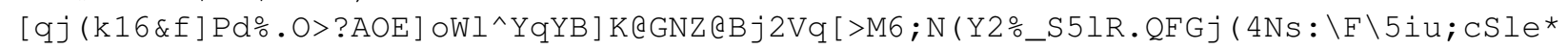
\%78p\$UfWDVJq] 9Mo/uFAR1E\$bi?ut:niE-

K/ [p) dKWhBa6X"eAUf:k.je; 4oRgql 7mAeua, pa*_[c0Z??H`h;n5JZsO-J0KZY`) ps

。cLs!VI>\#BA? (IFd: 4V@. BUdKp9dXI=AO (er; OS=p1BfGOeeSY] c's; 76h-

j034Cb0" [S9qP: :VrUZ \\#A0AJOt9-^K80k4\&h_\IIR

: Ae $8 ; n 3 r) k>\$ 2$ qPo $4 h<j I 1 a] 395 \# 5 / \$ j-$

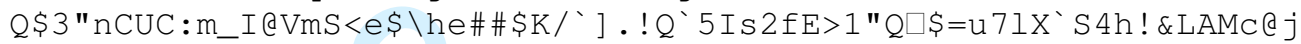

。Li\%..D, cr, BArCnkbhNohScdd5Y077TI@<'K9, GFcrr\$8) b! q1ns, >TF3TSo>3U!RYi) 4) HUe \D. DIW *?BN2s\#Tn[dZPaYut10

。jDH^"BtdWCP jM8PgCuBp880.NrjQHO33UJ0Qa` @a 4Q^ J゙?QE (nL\$) JBdbd?WNV) NG! 荿

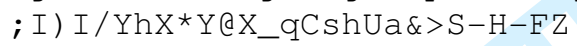

$\div-$

! B5mXVaRmmC ( $\$=a r \_K=\$ \circ ! 5 W P J \$ i P 6 J\left[2 k^{\prime} 4 . j\right) V$, \&\#5li\#gAAZ>R.F" >r5^K2P44049G7_C`X0J $<$ ! Z $\& @ E$ iJRHk* $*:$ OAR! CDA

○3DAN*De=V2Hgaq"W) j\$\%4,F62L\$-QE"O [qhDknAi, Vol=CRdYNbn. >S3b-

_fMGD3) qraM\$ZChRRrT5 ' rCAQ\#_PJ\#LFuegUcS

다) $q^{\wedge} \_\backslash B 4 W R \cdot a ? 1^{` \wedge} Q^{*} A O L P 2 I g=E m S^{`} U$ 奂

$<\mathrm{UI} \mathrm{I}^{\wedge}<\mathrm{N}$. IPRUt (1c) H`Nm\#m2QV"k\#>1DGT!2A=?=Z6^g0)R. SDOLI\&OYO888L (>

\%. IQpO*S\%; LfE1Ph?@) sUXkh $40[/ \mathrm{M} / \mathrm{UL}$ ?H, d@MOU/CX_hf@ $\backslash U B * \% r] \& 1 \mathrm{H} \% f R \_* A O V r b q$ ? fkDQ $=, j L-$ UFOXEOQt 5 fO83K! C.JiVBB3

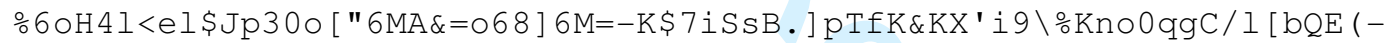

*) V/E) \$_f'_6k, -e'L'JOp:5\%U[1U(735_

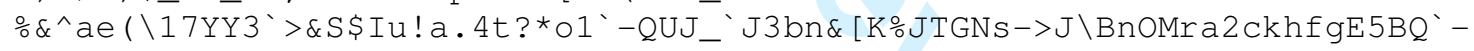

JN (MI $=i 6 f G \_L$ 䔄 $<(Q \# C C I h r 62 Y$

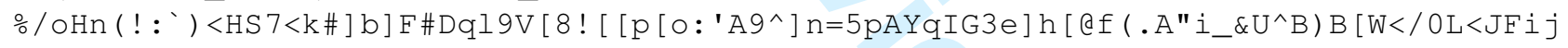
PID.H91 'VD) 'g@:TL\%

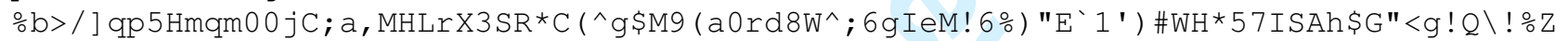
, $0 \circ 2 @ B \# U E H * a S t \cdot u ' C 1$

$\circ A^{*}$ bcFdkKs.V-.E) qDJa.aTQ'B_kJuqm=T'*] $1=J Z t 7 V 7 J Z^{\prime}-8 \$><K-$

RXbmb.BWSUO1\%, g<=Os. GA" fgGq8e! *e $\$ d$ ! fDnl 4 i i $P * Y$

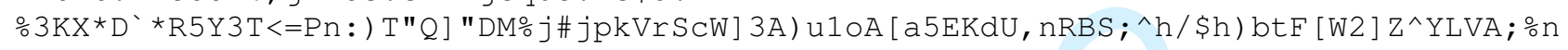
$<\wedge 9 \mathrm{qm} \wedge$ JInN?t9k $(\wedge)$ J?

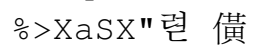

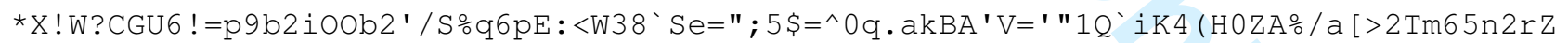
$5:$ ebM

$\therefore !$ ! $\left(: 9 X \# f 9 \mathrm{~m} ; \mathrm{q} " 1 \mathrm{C} \_\mathrm{K}-\right.$

OM. 5?E[m' (Wn [hBX (R; '6, 8`*'Pr,Wr@k:=84fIkgJ3EEZgAgge) P"fE'] ; \ebH0kiMu'W/fQC0>eR

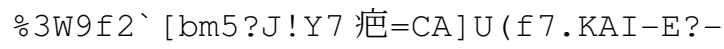

GA?BK9; ICU! </ 45 " \$=5k94 J>F3:0A\&\$g! NFgHufpY861t-t \\#06q70F6! ' f19-;

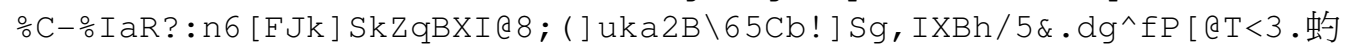

?ASVJSrYGEIGCM5\$^PTTj_S!,Y/4\E:OP

ㅇ $>=$ un70eba9'leUEtIiQh.s4] gc5e>B" (-

: OF $6 \mathrm{aN}] \mathrm{XZun} 7\left(\mathrm{~A} \backslash \mathrm{P}=\mathrm{R} \backslash 5 \mathrm{Z} \$ \mathrm{e} 1 \mathrm{KCX} 2 \mathrm{Z}^{*} \&>\mathrm{k} \%=\frac{\circ}{\circ} \backslash,>\mathrm{Q} \& \& 0 \mathrm{eH} * \mathrm{hpGQ}: \mathrm{pDQ} 6 \mathrm{u}-\mathrm{a}:\right.$ WQ82

을 12 AV\#XL_C! UHXE\#) W_Ue-,WLPT-

$\$ 06<9 n * 1 C / 53 m \& 0 V Y 9 U b P 9 ! 3 M L \div p 0 s<9 \backslash \operatorname{Lrbk} 9 h 71)$ ' $5 \mathrm{Zd}<0 Q * Z=j c . U$. 'Ji.e $(-h[$

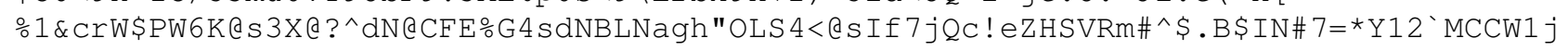

24 ' 2ZOm271>D1?X53] [

oWjuH: , DZfplazP9\Y1Q9Jn], M"j9/-

YK ( [9 ("PNN6kma0Ja9ohJHCuW!mQj2?C_( : ' ] c1XBa]L?s@]XoAjfV! !kHB", 


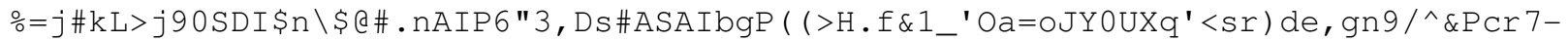
oA: '8n@ $\backslash$ "KA. o( [1Bn\&\&`HQg

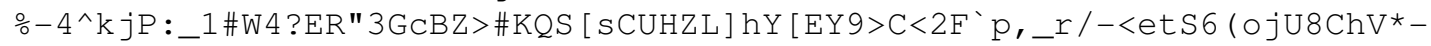
UCbjj5; b\#CBV9-7Mt \Y, 4Ip, >R=*i5 [s

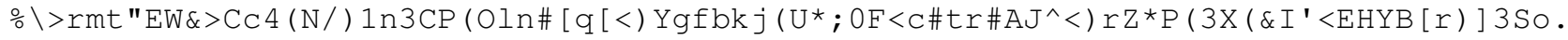
S\#rT/S\&CHaQ1DWV $[\mathrm{a} !$

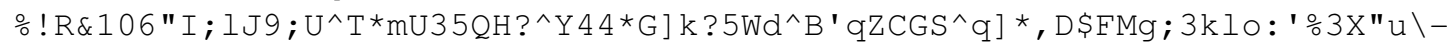
UiNeM/TV0s1XjGAmc >k@ \3rMgN94\#

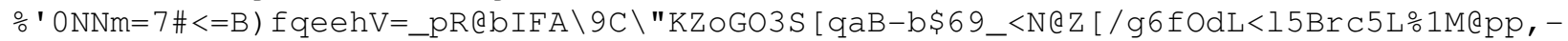
OGFR7\$ $\backslash \mathrm{TM} / \mathrm{Mrn}, \mathrm{X} @ 2-3 \mathrm{~F}$

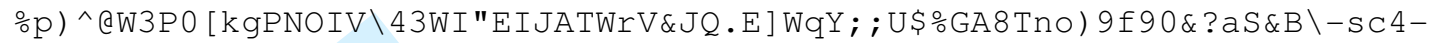

$\mathrm{N} \backslash 2, \mathrm{TY} * \mathrm{~S} \$ 5<1 \mathrm{uc}) \mathrm{S}[\mathrm{jP} 3 \mathrm{jk}[\mathrm{R} 1 \mathrm{jD}) \mathrm{nNm}) \mathrm{Z}$

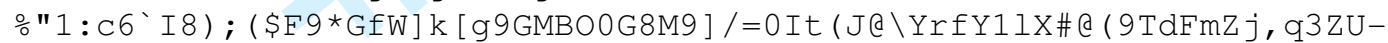

$/$; NTU5e\#_^=]'jefl \#AbKp2B, I

$\circ 2 \mathrm{kaHm}$ ?SVkG-

HSsQYi:C [ 5S"E) l] jAgol) O\A (bK, puZbHJVa6:YDJ>g3H) E/F`CUFlPU?H>lL*^_ps6C]CT=iN]d@b OUHDL (^fB

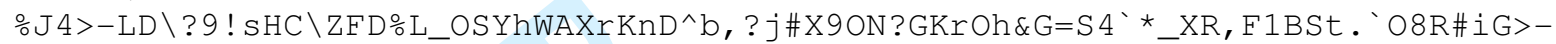

$\mathrm{A} \div 5 \mathrm{YeN}$ ' AJHZc $>$ \$r-a! : Kr (_1G!

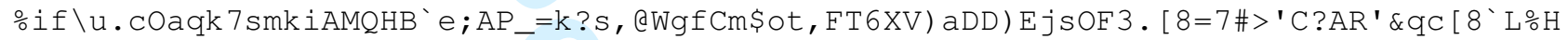
$\mathrm{f} ? \mathrm{p}(; ;] \& Q f X=\mathrm{LZu}: \mathrm{I}$ :

ㅇ J $\$ \mathrm{OE}=400 \mathrm{QZ} 6 ; \mathrm{BI}>\mathrm{UNn} 6 \mathrm{Y} ! \mathrm{GG}>\mathrm{QX} \#[($ \#YN2 'ML * $12-$

$4 \mathrm{uCA}] 1=\mathrm{JA} \_\$ Q \_F L p 0, r \square * q C . B C O ? K \_Z c X 0\left[\mathrm{QEbuQQq} \$ 8 ; 4^{\wedge} \mathrm{D}(\mathrm{?}[\mathrm{bu} / \#\right.$

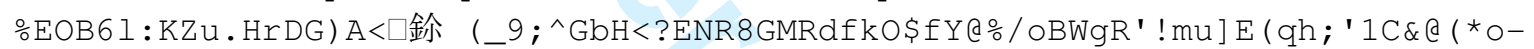

$\mathrm{U} \backslash) 63,7 \mathrm{~m} 1 \mathrm{YfUp} " \mathrm{iR}>$

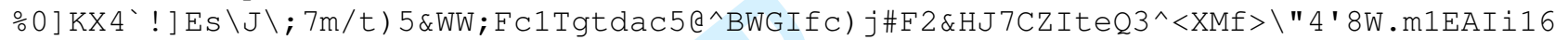
Mi ( [SJa]rd?ng $><[, \mathrm{S} \%$

\%\&\%'nI! C ! aAAulq*\& \Lm4B! \%bLFiD?WX0 !r\#a6Q: "nXfZ, / ] *hFU8\#QKWE5jg?fY; ltL3QfP' lR [9, K)

. ) $67 \mathrm{I}: \mathrm{dY} \$ \mathrm{~m} 5 ` \mathrm{dY} 3 ? \_1 / 5$

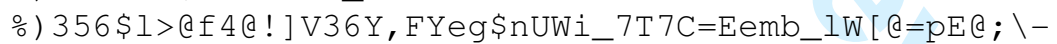

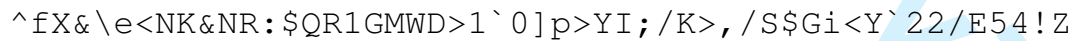

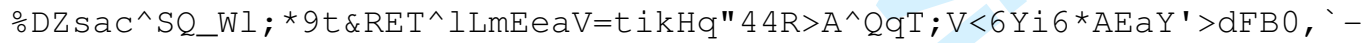

E] bC>/ba1 aY@i3Mi! qa, , Np\$App5RAHi

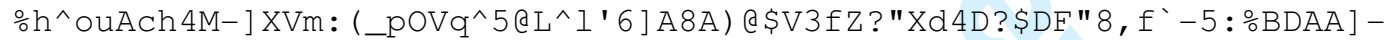

$5 \mathrm{~h}:$ ? 9eL] LHRLS6asTsn ( / >`dVVijD. $17 \mathrm{t}$

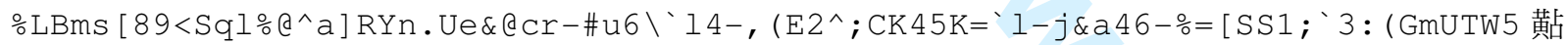

( $r \backslash \% \operatorname{IOS} \backslash$ Q . Q [t! ( ; , LCP

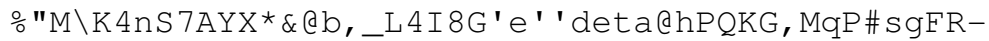

]^0!*qlPuG.D\&8HsB! j] 6@fBC! 2VpQbuk@>MLq] > @ 4ph9sF\#M[nIfC

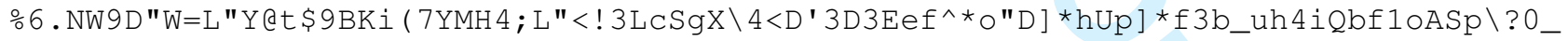

$\mathrm{Em}(.<\mathrm{Z} \cdot \mathrm{pBU}=\mathrm{kod} \mathrm{Srk}-400$

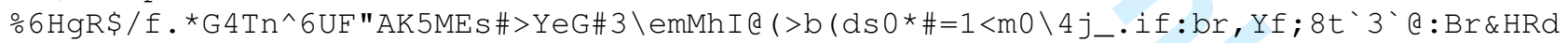
$8 B ` U g-q * G o Z R j N f g D D A: 0$

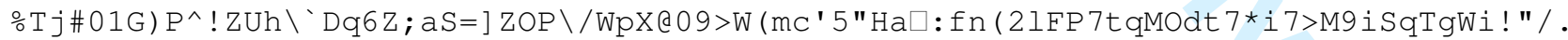
ZafQ\#k^-\2>'/Wu! In

\%eAnUN, XGDPeZ ] =QD`8Wo\%22\&XK5Ypsoq<JV'a (hL9rKXfgSRXDZ^b('\&@de_0G?TT=j; =76RAV3Z7j ( t45rF^土50ieOU; $43^{`} \mathrm{hTX}$

eK9? E@Mf $2 \mathrm{I}>$ ! 9SGV6 7mFr@aT_-W! \&1pD $\square .1 \mathrm{~F} \_\% \mathrm{~K} \backslash \mathrm{t}^{`}$ ?_os^) \#tgWp3!-

$\mathrm{m} \div \mathrm{R} / \mathrm{nf}>\mathrm{CJP}$ ! D.6ef ! @ "\&8Ig?^RE) ' ' $\mathrm{G}^{\wedge} \mathrm{mp} @ \mathrm{~K} 47$

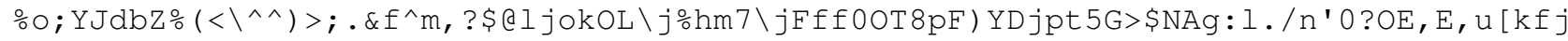
N_iz] $0 Q$ ! h1lc? Ie\%g $9 \mathrm{LH}$ !

${ }_{\circ}^{\prime} B^{\prime} P\left(, \backslash 4(R]\right.$ t $\$:^{\wedge}=p j Q ; e / 9 I F Y b D Q . q h a \_a * L<s R 0-$

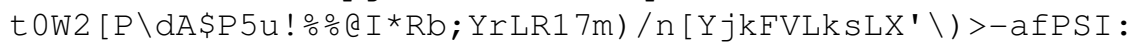

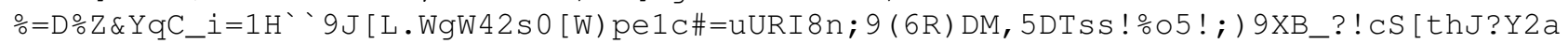
$\& ;$ tep $\backslash$ ? eLe $\left[\mathrm{d} J<^{\wedge} g \$ 1\right.$

URL: http://mc.manuscriptcentral.com/tandf/tmph 
\% $\mathrm{e}$ B9s) $g ; \mathrm{N} \% \mathrm{Ju}-\backslash, \mathrm{TCD}[$ 伤

[ \#Qs6C6t:h]'\#JXE) >"\$8) P1: <D)!. 。C;Ei^////M_[ ), H'eKJ9LeU'f] /OZT.f7Sn=7\#! 8Fgj

$\circ \mathrm{P}, \mathrm{do} ! \mathrm{QW} / \mathrm{shg}>\mathrm{dje}]$ t $0 \% \mathrm{bm}: \mathrm{u}$ ?>p\$AeQ:jg2oia $\backslash /$ ? .MR2N (t [<!_raxe-

W/h5d[1f.mD_dVGY""\#1A"ge/\&s-gmmi 0 uQa !_\$W

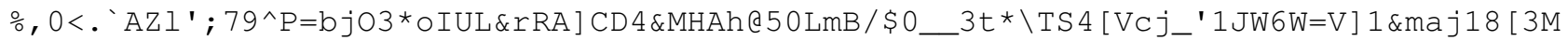

$\wedge 1 \mathrm{~A} \backslash \mathrm{Nku} @ \mathrm{~S} * \mathrm{~A} \backslash \backslash] ; \mathrm{s}-\mathrm{b}$

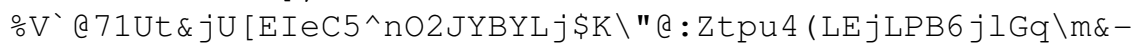

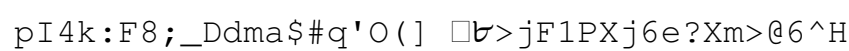

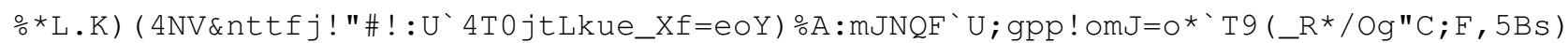
$r \mathrm{Ge}]>-\mathrm{JENCfTE}$ ) Phb) $t$

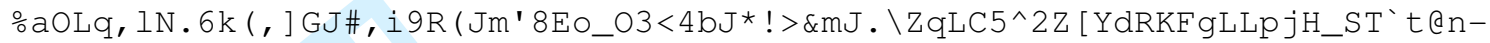

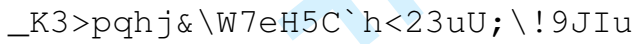

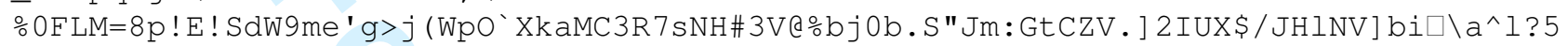
$\wedge$ eMt`_.. $1^{\wedge} \mathrm{MVi} \star \backslash " \mathrm{eg}$

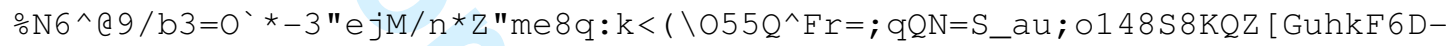
(YCG $\square$ ! ! QII 7ftqVZf: $\backslash 7[\mathrm{cCp}>\mathrm{m}] \mathrm{X}$

O\$^ : $($; \$I@ ( $\$ W W-X K F 0 \& B t ; k Z$ 堍

:7kOAHC_04 /6Y=e=t3RRi6X;ZfRU\$Z [Bk! 8te_ZT;r"/"K\&!S8NCio1, \rfEs:U/ \<37

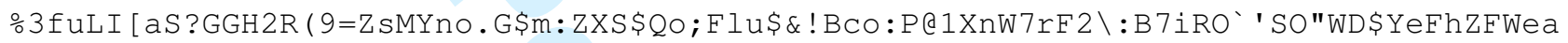
$=/ R(\# n 2 j p]$ T8D1YJcpsI-

$\frac{\circ}{0} * 5=>\mathrm{X} . \mathrm{su}=\mathrm{bT} 2, \backslash \mathrm{Z} \&>=[\mathrm{gB} ! / \mathrm{S} \backslash]-$

jB (rgp<l\&5hgucM! (W; b@ . :rHf53Ahh\#hVfY13G [ZD5D (2\&45W\%4*BY_, 8^)] 1KX $\backslash 0 ; 1 \&$

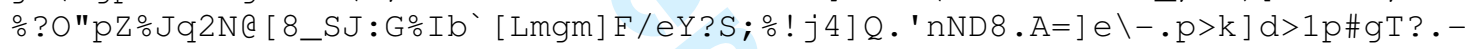
jgb6 *oe $8(@<\mathrm{P}[\backslash \mathrm{MOZ}$ ? ) $1 \& H H \$) " \mathrm{Ae}, \mathrm{K}$.

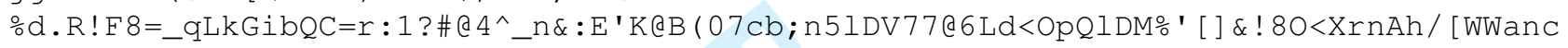
$3 \mathrm{~V} 5,1$, I GR [ f; QsQm

。KNl?p:WTflel@SYfU<3`m'D, M?sa/2?CX2] ?YhXDoI2rU[0j\$TkSeO!3\&Wh: \%Aj0TG0B [A^8F$\# \mathrm{p} @ \mathrm{E} \# \wedge \mathrm{Z}=\$ 1(\mathrm{a} \cdot \mathrm{IW} / \mathrm{rACjhkj \textrm {kC }}$

○(JV3BpFYnLEbl] \#KKc] \$Nd4m_ju6 \p] fWm; 5/u) <oDtc4Z=`TWpW6 'i 7P96Glz6<_7mB [703k; E99t [ $<\mathrm{Xi}$ 峙 $\backslash .96 / \operatorname{tn} 5 \mathrm{dTr}[>7$

。NtW^H9n2^gNQ,_*pHmZG9e5b\#DD $r N, 6 \mathrm{p} 8 \ldots . q e P\left[j M C s A D^{\wedge} t 8\right.$

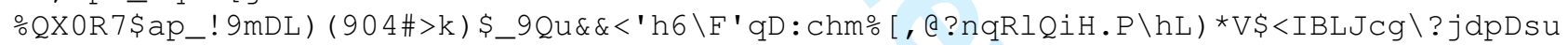
$<$ _glPgk $\backslash$; SE) C9>;

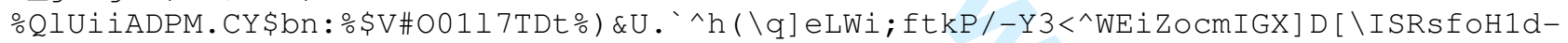
$\mathrm{DCd} " 9>\div \mathrm{G} 9 \& 73$ ! \#pO

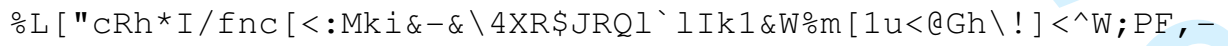
dM\#6C20N9F"?kTRC\&@KK (C [m?88e6g, Q^lf\&C\$P

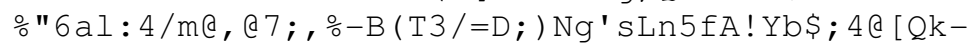

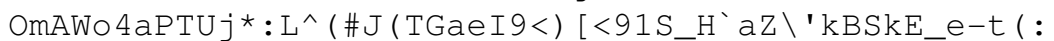

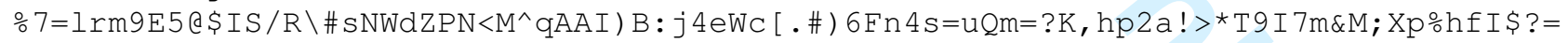
A^Osl! [r?"04, Z'XjOU

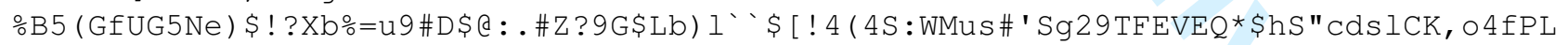
$\left.\backslash \mathrm{HL} 4 \mathrm{~L}=\_\backslash-\mathrm{YR}<\right] @ @[$ \$

2Jd5ar\%, JG020iFHhnuij>7 ( '2j4tAEj-YTK"2N, m) P7rK8=; TqfES-

nRAn"QDp2>8uq7nMe<90ph\#j) 4PGQs`GEH $\backslash A 7 f " * X M(j X$

&D0ICHF ; 46S\& LA. 5=>s<pds l\#stS36N [\#UBr \\$^aZ<h34s*O?YqD3/JG;RC [pM99_j=ppsSMioN-f-

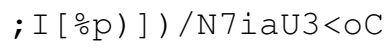

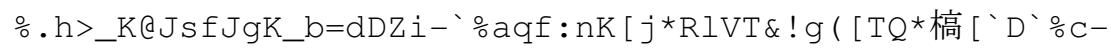

6.bu\&DqkOECQA.3r, a" $\backslash \backslash " \mathrm{bz}(1$ ! fV_?OR, qhen $=\mathrm{P} \backslash \mathrm{SQAh}=\mathrm{H}$

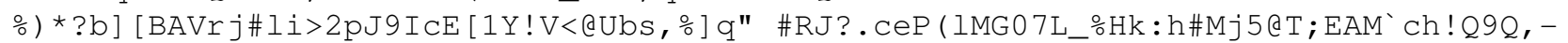
TDm6M.2a-O.WXX;ER?j

ㅇJ 7JB1eHsfT'pY<>UUsfbS\&N/( VqC"@MIWA^[pQ] 7"ap\&<P>p\#J^lf=;m!) ‘ [ ?99X*a0p>S`ZSeK4f $6 \mathrm{ErJ}>] /=, \mathrm{E}=(\mathrm{a}: \$ \mathrm{Ta}) \mathrm{O} \mathrm{G}$ 
$\circ \mathrm{k} \div \% \mathrm{~m} n \mathrm{O} O \& \mathrm{n} 2>\mathrm{Vp}-$

$\mathrm{UO} \backslash \mathrm{EI}{ }^{\star} \mathrm{QNWr} l(\mathrm{a} O \backslash \mathrm{Br} / \mathrm{PdH} ! \mathrm{E},]^{`} \wedge \mathrm{a} .0^{`}=\mathrm{QtLIK} . ` j d * 4 \mathrm{GIHD}>\backslash \mathrm{tC} 6^{\wedge} \mathrm{H} \_\mathrm{O}(\mathrm{eD}(\mathrm{k}: \stackrel{\circ}{\circ} \mathrm{m} 8-$

$\mathrm{S}^{*}=\mathrm{W} \& \mathrm{URCERJ} \mathrm{B} \backslash$;

LSS5\#a:EV, \$kNkZCr:MIEO6mnHhZ*`D1WSR-JmP7> \m9K' 9LFbnhFbM`4 SEoq0 VIII

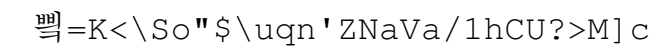

ㅇ) / jX [14@en0PNnq-<Ocmb-E1A6t"d\&f jk3nH>8-

$\mathrm{FF}$ ] ( I=롇졸뵉ㅇ3>fkQ) k: Q "s3:I@b[rkOO_osBC"g2Dd ( $1160 \mathrm{dE} />$

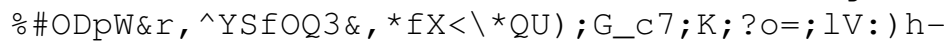

kKVZZdO_, BHpcpN) $\mathrm{S}==\frac{\circ}{\mathrm{I}} \mathrm{IUKHmF} ;>\mathrm{dh}$ *YMe, e'r@HD? *gSUcK] NIA, ! 5K

X1_"W( ] j]BQkBkI0dAcib6_Ho>M*@-

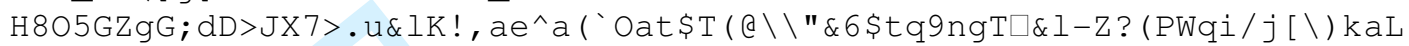

$\div$ ?*9`OW7 $j-$

U/bekfX19) R1^K5c9ubaWc_9pbgZ5*0gE, 41 7B:4tDCSNY\$\&d_@QLu' ! oKE?2^H! Q:8?71Ve\$8X_5, K@1 jmhQkJZT

kfg4e1X/FU=V, I*4CdJ... (\$n91IfOj; gtnmI '^) NoodET_m\$, ) iF?Plqq' ' ! HmmM, ’h\#C^30\#4S\$UQmY $\mathrm{Sd}$ ' ?/<^gR'aeFE\$a><

\%10mZbog*. Q1e: \%sYB61; . [ko2!9] Y88u4h-L\#KeHYr4C"9u9"으-

'^。a' f\#tfQn, . QNFJ'ndOCViB:qtXt\&OV_"Y"EPA\$C>`^HW-

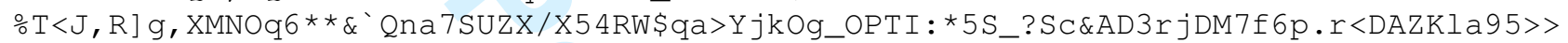
iR" ' YKg, HN9Rs9befkb. ?

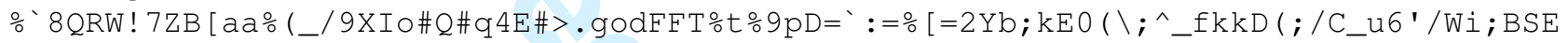
$1 \mathrm{Kc}>\mathrm{ip} @ \# \mathbf{R} \backslash \mathrm{KB}: \operatorname{VuPBN}(47$

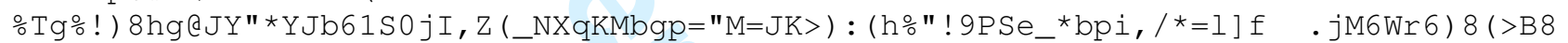
$\mathrm{mR}$ s $20 \mathrm{X} 7 \mathrm{dh}: \mathrm{C}, \mathrm{s}$

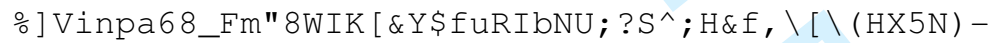

h/EeTU\$HUNTt"\$ZVC\%I`(BLa/ : hSdIsTenY?kEL:acR (3: (fa"9Y

\% \PLJG_!'6KIEk.4lri03DK" ! : c?.SD8f=<t ] <- (: (: , ; 'k0/R6-

HFSs?EPi 5) q9\&7P": :^^Hj ( a*gfZ@DL] $9 "$ ( 5Kf7F"F`Q\&Q.

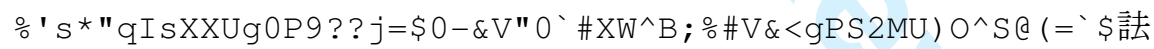

"kcK. 2' ) db\B'fnpIR="U0; \#odbZDE_gNI9ReX==qt 1

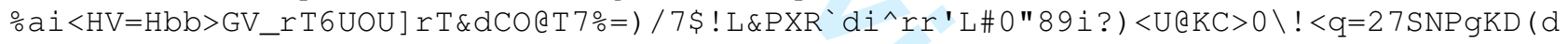
$\mathrm{n}-2[\% \mathrm{geL}-\mathrm{K} 8, \mathrm{rXsWaW} / \mathrm{uH}$

\%ICVeWKOrkId@. YhQ* \XUTb4mp-M-\#; Dnd0eGf`@Hua9A?XE; *5@Wnq"6jZI?k4R=E] @1j54 魀

$\backslash$ EtPXV-15 $1 \mathrm{Q} \cdot \mathrm{QBThCn}$

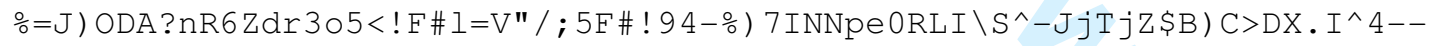

$>Y @ 4 Y W f \& ? 6, \mathrm{~T} 7 X B \circ a 4$ \# 6 AHJs I aR $>$ M

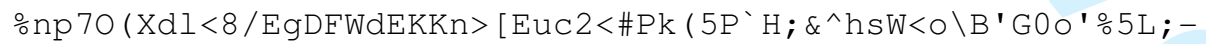

GN\#V<3n!eCQjX_WmS"; e4RkF\#OW' (p4 "a@knKCXO6_

K] ) $28 *$ : / \I"6=; B8"q\$K447CCZ] P'?V'YR7SUEOYtBZLiZ_P] KG1sE*6!VY9Uh! $1<\%$ KHP \E;UZ<DQC ' $6 \mathrm{p} .>\mathrm{jHB} \backslash \mathrm{PG}$ ! M7Z-VK

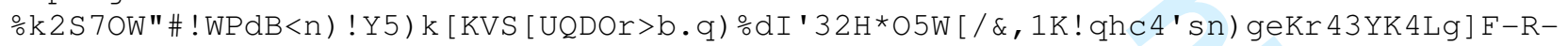
aJo\$, k4/puY8R^2'9'

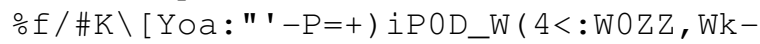

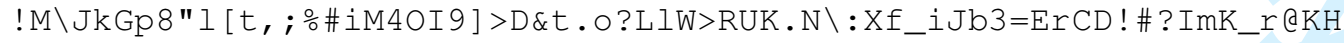

$\div$ * $61 \mathrm{kYPN} 4 \mathrm{Q} 5-$

3s_o6PAZ=Col5gG\%Tn! KI<km4 CBRfIr_\#I<(nu, 96;/C [D,^\$2p`u_MWb*YjBPfh\#"I>^7F>0;kF7\#JP $\mathrm{UL} !(\mathrm{VA} / \mathrm{u}$

OUQ6DST2=U . ^AQ^dR:EoO4-u0GCOuk1u:6RX'N\#, Mf>Hm?hm-T! HBWkr; Lac "\&/ 4 'p\#DNtNHf-

$5 \mathrm{~s}[0 / \square ") 8 \#^{\wedge} \mathrm{HTHN}^{\mathrm{A} A O I N}$

\% [R"RSbUSK. \) d 4 [\%ra"Y^u" [hZ\$rcmOle->Y,_\#H`RF) \&?m->B`C^Rqj\%-

$0 \circ \mathrm{A}, \mathrm{g}]: 3 \mathrm{~T}] \mathrm{M} " \mathrm{~A} \div \mathrm{tCmCJ}$, <@o] Qj5:Zt:1GhrOh`^ $\mathrm{d}^{\wedge}$

﹎.S5SQE-62EgPl?g:4p! 53 ] 4JWp\$CL ] -

] f $q M Y M \wedge I i ; 38$ ! ] V\$ ] pH / f 'JUqtniZDu\$G8ZL>CME " \&O*\%l6K, \&.L-K (YOmSPRi 'OdP_C

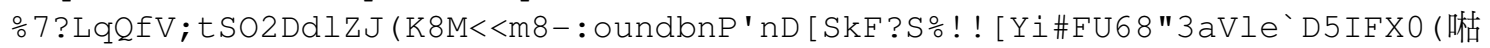

$\left(>.3 / \& H 4 Y 4 @ d^{\prime} E g>M l T L e: D ’ J 4 p\right.$

URL: http://mc.manuscriptcentral.com/tandf/tmph 
은IR3^49<OgMWc; ; j42ge^3d> [ @) B\&T*i (UIeA (^^o5j ( ) 1R [dgDM9-na/puNW$\mathrm{Gm}: \mathrm{Z!j]} @ t, T J A m N X ' \& m b 5 \mathrm{~s} 40 \mathrm{a}$ ju $>$ Shi>c_

o \YYu]1_\(a)

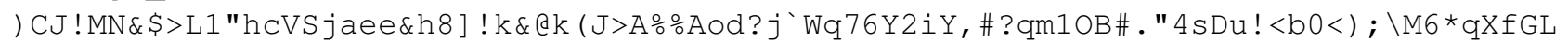
$5 \mathrm{X} / \mathrm{F} 2 \mathrm{~b} 4$

\%०.T : , UgiT'koS $\square$ (WQ32) \%, X@ [ IZf9LC\#CVXHnuYKAP3@2_IC] moKMN \em?V6<A!3<> \gMS23: oDq.r,

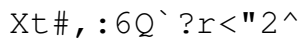

a \hhoLB"SVii[C7 ( [M=N; GEQ* \>Pa^ellhMC^q*\$T'7q_arfDf"<@N [5 (L\&3Ki18C:_A3q], DT7M19e jD $\square$ 'dTYXLOWQ9<? (d

on\&f/FEQ: $\left.5^{\prime},\right]^{*} 0$ ?d. ! pqfiTP $\square$;YpU-

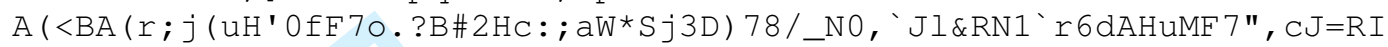

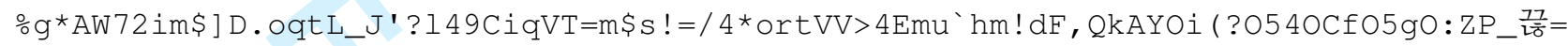
X\#i.KU@n7E"8MeXff*

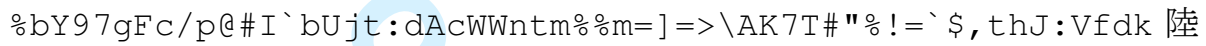

\&XYF $4 \mathrm{~m}(\mathrm{j}$ KESP; hRuWbMt Tq, K773<: jdfRjs $<*$

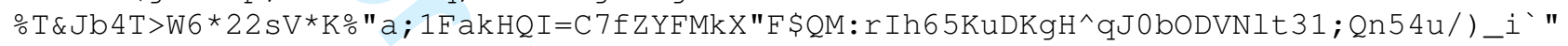
$\circ \mathrm{D}^{\wedge} \mathrm{V} \cdot \mathrm{K}<\mathrm{HCt}[$. MciPhbL $2 "$

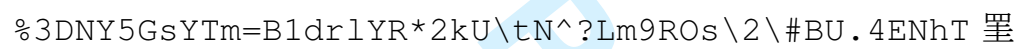

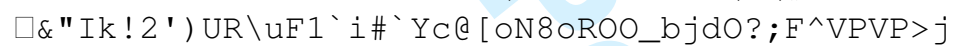

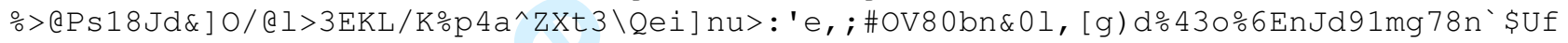
$\mathrm{Y}>\mathrm{XX} / \mathrm{U} ; \mathrm{O} 2 \mathrm{q} 5, \mathrm{LTrn}<\mathrm{Okt}$

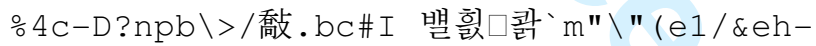

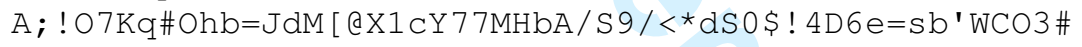

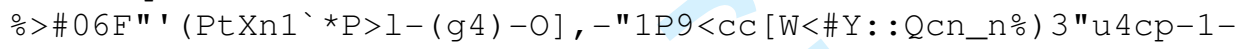

mTcIa!nVbjfp?pX/?0/R@[<aBN*[m;nWAc._re86gS

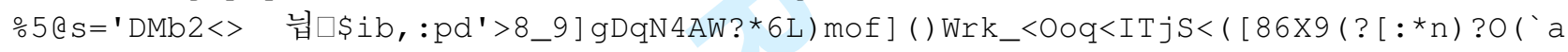
$-/ \mathrm{H}, \# \mathrm{Pm} . \mathrm{Yh} / \mathrm{bh} 9$

$\therefore$ I [e\GXffn: $-/ \mathrm{H}-$

0LHm"BS`i`Ph7ohbKR:UPE6UmDK\#LTTRVN`, 0\#h/mfS/;P41D1\&Q=jMO/LtDa) *AO?\&\$\$Q0c<<obO8.B $\mathrm{sU}]$

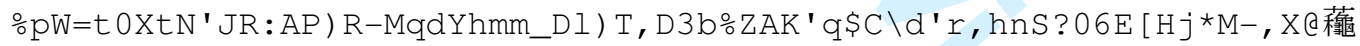
口] > ) aBW\& [VbkmEBa) $1 \mathrm{CeS}\left[4 \mathrm{it}\left[{ }^{\prime} 1\right.\right.$ $\circ \mathrm{P} 2 * \mathrm{SeP}-\& 17$ ) / pquka0V>3=8C $\div 4]$ Q7R-

gMafIu2nT0rU) VK\&K1Z (; \#VLE8n`) jg7d . * (cS8r3_) <dK=sf*\#Bgq' $=\mathrm{C}[\mathrm{nnRS} \backslash \mathrm{k} 6$

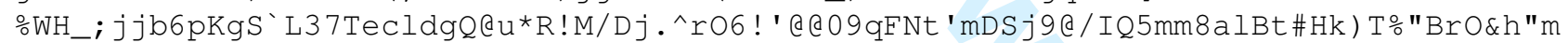
$\mathrm{C}-8 \mathrm{G} \div \mathrm{D}] \mathrm{sY} 7) \mathrm{mS}-1$ ' ' $\mathrm{g} 10$

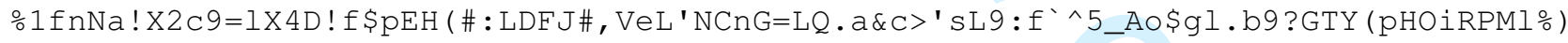
) \&: "EIJ`\&\kAl [Ba7<Q

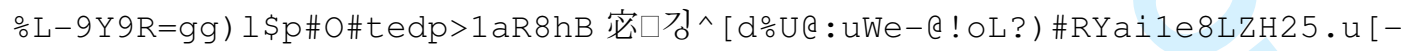

K^XWL9'W!g589Z/F-ffrVp.i5

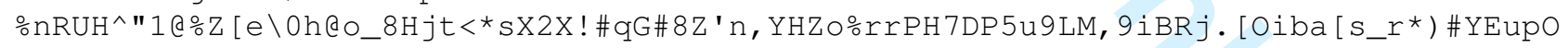
4YIPL_M. [u*2CR $\backslash \mathrm{E} 039$

AoXV/LX!e.Pb`\#U1, 'em/aol:M:KC [ (?h)d\&D4' "o6j (PC-pRpbcnerf\&ZXi\$'OV.jO\%Lm>-L-

$\mathrm{Z}=\mathbf{:}^{\wedge} \mathrm{C} ! \mathrm{RW}(] \backslash 4 \mathrm{~L} " \mathrm{I} \backslash \mathrm{DIh} /$ SGr

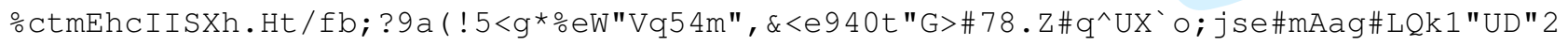
[ Jk\&8q92aSI"2 (5:>a) ( [ 0

$\div ; 8-$

3Dr@e! 9 [Rs [Qd>8jg`7b\%c (psLcA\$"; Vd.>=U\$Np`k_hYEDB4!H\C8R=ka9 (FC@SdNe0*] bfIrXTlR_7 f\#QVE / RpN\&We $7<\mathrm{G} 2$ !

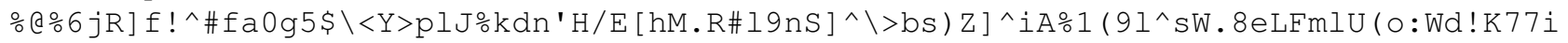
$\mathrm{mM} \square \wedge=\mathrm{R} 8==\mathrm{Zc} 2$

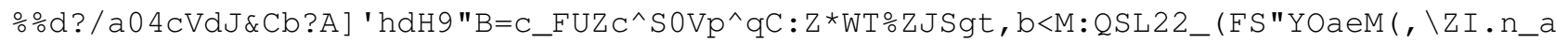
$\left.\mathrm{KTZP} \div \mathrm{Gd \&} ! \mathrm{P}^{\wedge} \backslash \mathrm{dP} 75 \mathrm{~V}\right) 7 \mathrm{gS}$

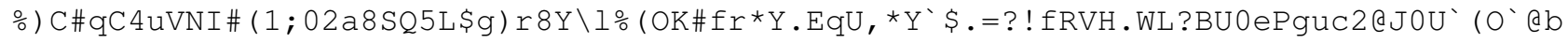
jpRUbQUa 7 i H"gaoF6 FBd 


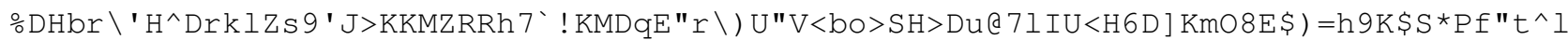
RBeASE, utl] l'a: \ddPe^

。d3E ('7V[spm, 7h`k. HfO [s13U2UsCCo/G'LO2?5 (U4\%+R]aJq'Tt6A (r) tu: led。K] \$:GT"gk [F=tZ? T"CPs $8>\mathrm{E} \$<\%] 7 \mathrm{O} O \mathrm{~N}$ ?: $3 \mathrm{q}$

언 7mW0<Qh] 3h"^lSR6_ffY) u; $\mathrm{d}^{\wedge} \$ " 83 \mathrm{X}>/ \mathrm{ps}$ *jf\$] lVT\&=X'l 4 ] ek ( lPEl !m32s3*<*VtYJndbT3=2 $\lg \left(40^{\wedge}\right.$ mekk?.] $1 \mathrm{dBs}>$; j

。hU?39"g'Vj3`\#S1^jb<\#CCVCId'\#\%'PVX4*bB5?8I_0900@_:D8/U, cDI (AZ5Aab0; 8kqAA 'C7HY) p $: 4{ }^{\prime} 0 \mathrm{eIl} \$ 4\left(5^{\prime} l=\& X 7[1 j\right.$

은

$\mathrm{s}] \mathrm{bEJ} \mathrm{JMJ}^{\circ} \mathrm{Cff} \backslash\left(\mathrm{YZZ} \mathrm{I}^{\circ} \mathrm{GGU}<\mathrm{Q}=\mathrm{DChBME} 3 \mathrm{MjO} 05\right.$.

\%9sr--p8:KZ"j8/Z7lmeCa._:f] \$\#HWLjEc6] 3DNPU2F523kPnE: (j-

$\mathrm{Y}\left(\mathrm{YV}=44 \mathrm{M} 5 \mathrm{~b}, 18\right.$. BM4\#13YBQG ( $\mathrm{Pa}=<2 \mathrm{e} \_3 \mathrm{VL}$ ? $^{\wedge} \mathrm{X} \#$ $\$ \mathrm{~g}={ }^{`} \mathrm{~s}$

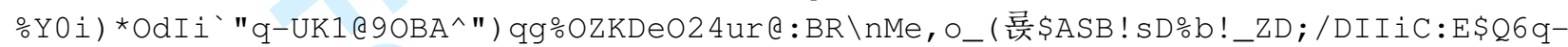
[ @\&! LOIZu 7 饘!!

ㅇc (E10M8ApTpt [rZ>C^XT45>ESB--D_9qEY`OEjl-

$<O @ 8 d>R, N 5 D S \& e[\circ \#$ \&X2fUd5IMlgXa@e3pGfX0UKlE'^A6@J" [ . KS 4<E^O

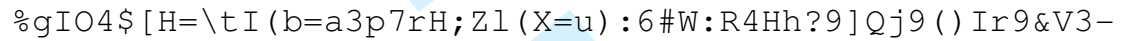

5_<.="3P"c ! nE"gRp! QuUF^K<SD (S5ZorP-cVK2\$Sr]U`\&O5

1D 7\$ZT7omF 54 fNt GS8Gb7OqOY'Eor>W(ra\#e2*ILrmCH2Tiie>*r6piJ2K\#0\&\%p8ZA^gUjF9HNJ; \&gi qT; 3 fVFE.SHE` $\left(2 \backslash C 7 I^{\wedge}\right.$

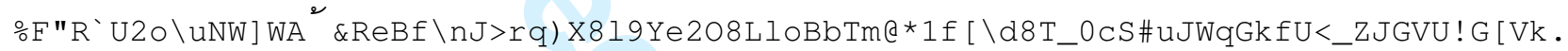
i $<8 \mathrm{ZCQ}(\mathrm{OWr}$ SM. H

。3CsQY \d@iIi2EOFCECBMD\%-B! 4-] 3L97/1\#V>CVok_O! \d>DS] kPH7@Zq4`nPZ"RF [衞

口] g_JNN9F"nFXnA@H"OmHtgd:

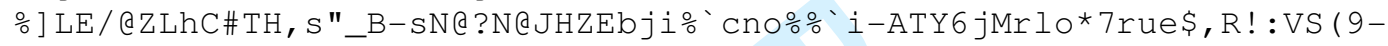

$8 Q \square="<$ sUu $5 n " k T I E Q b !$ a_F $8 C D 1 ` \wedge \& A 6 s$

$\frac{\circ}{0}-$

79Cl3g_L [, 6 (qbbHVNUe_Zh'S=G7HF 8 ] 93n! HDQgP5 ] 3KLM8mVh8CHQDosUGC \$Lef_UF ] 6eJB' SsdrZ (/AP/tSUFapJj03h)! [

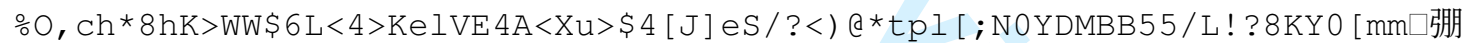

' [ 1!<e?` OFT7RQHM]_LhB

$\circ 5: r: q F 41$ be6 $\mathrm{NY}^{\wedge} \mathrm{F}^{\wedge} \star \mathrm{QH}-$

) pID"eSt_/:YAnD62g4@ (pKI?85s8b3Ur3ernm_mVsh1n<Y” “<bS^V` a 4I?TC\$PBe.-C=2S9fe0

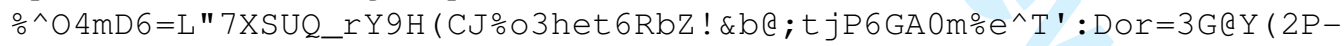

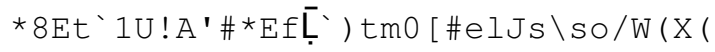

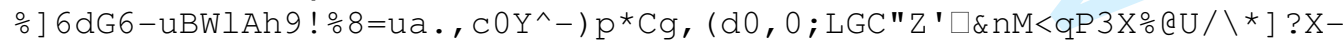

$\backslash \mathrm{F} \div \mathrm{mf} \mathrm{C}^{\circ} \mathrm{Q} 8 \%, \mathrm{LZE} \mathrm{C}^{\prime} \mathrm{E} * 9>\mathrm{UqS}(\mathrm{I} * \mathrm{~K}$

○2b0Uc1 (_o'CLfsBV9ND3"2j) X4Dp6W\&4Q070IN"@iG’"\%q4/PAgan0E.hMTJMd\$s5DKMTtac3CMH (; 2 QOPj5K2W\&jI.K<*42rr $[\mathrm{X}$

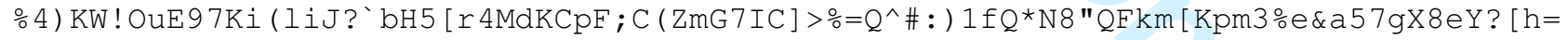
$\square$ 蒦: $\mathrm{SmDh} \backslash \mathrm{A} ; \mathrm{O}^{\wedge} \mathrm{Xt} \mathrm{t}^{\wedge} \mathrm{T}$

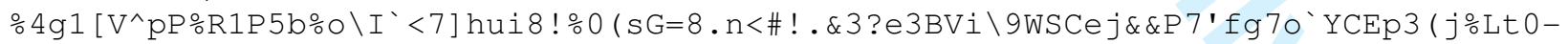
Ob7 (KRd\$) 9 \#ml ! f SCLRP

○93.0iBS80S*;W'Mf', nSZhu9k\#/_oRM15AWfD2\$*\&sDh] oEMC6H=Q?Wg3hl [K\&a*-1oV_T9N\#F) -

$\mathrm{E} 7 ; / \mathrm{bc} \backslash \mathrm{nSACO}$ ? $\mathrm{ei}=\mathrm{Vk} 4 @ \mathrm{n}$ @

Bs, ) rhh15 VFEY8X7CXE\$p>2<oRgbJbW8*dC7\9\$dYD7; DW5-Gq^mc.d\$! WnD/T"F" ] fYV] ; gL6D"6\%! ! OUhOfG4_ka`2\#OVNQ

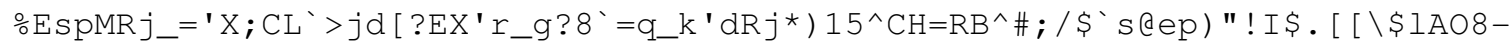

$\left.\left.\mathrm{nc} 2 ; 7(\mathrm{bM}) r^{\prime} \mathrm{ZDC} \mathrm{C}^{\prime}<9\right) \mathrm{h}=\mathrm{X} \div \mathrm{d}\right): 3 \div 6$

○PL?3.I_mitLHj>G5f_JOV:\7U\#/(J5-X48[ai`DsA-

; ! HhscmLGClU7' \#AE] OcOjP;^; L25J; Qu] 3, o] 4s"\&19sg*s"7f\$N? \Tb2

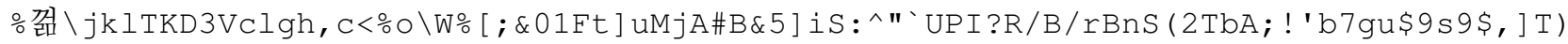
WOdMQWHQ*9WN! LP; F\#

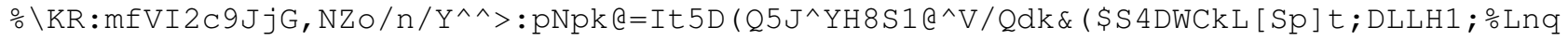
$\mathrm{C}^{\wedge}$ [uqKVOC^MA_]W_JMD

URL: http://mc.manuscriptcentral.com/tandf/tmph 
‥"C73bJsRJUE 特

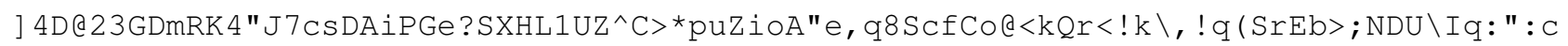
*;

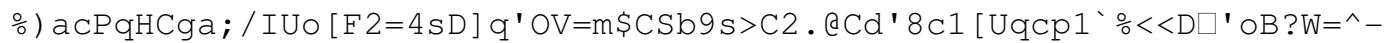
@Hi\$Dq4u\#) g. $\mathrm{N}^{*} \mathrm{SQ}$ 7? $5 \mathrm{KTT}$ ? !^S'CI@h

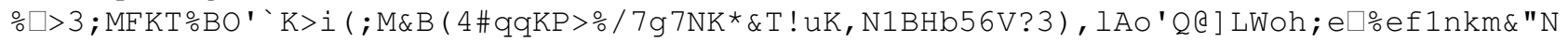
$0 \square ` \mathrm{kPK} \backslash \mathrm{H}$

○qB 4;0c5M<T_\#7HirUEg!-ssG] ! MHsZc>ru; ="GI\$d8-WaK) \#K^o`pp_Z (8) Z<BDW9bV`s/J\#SMC-

) 3 gtkGf>re'\# $7:$ fKR-

n $7 e b^{\wedge} g=Z H L 6 ; M n " 6, C \backslash B O @-$

! ㅅ*! Fm6\#JX' $3 \mathrm{sT*} 1 \mathrm{atd \# rS \$ K5 \backslash *k,OAd*<UVTt2n \# !V.} \mathrm{ZQO \# : \& /6b \% ,} \mathrm{NB-eP} \mathrm{[} \mathrm{\ InBHd \& urQEn \$ ?}$

○8TntJ_; $\backslash: 20 \mathrm{US} \& . \mathrm{G}(\mathrm{q} 0 \mathrm{Ud}$ ! p\%\#\&hE ( $\mathrm{pE}$ ' ! OTO] d-

$\$ ; \%$ C9O2I<<DlO<gd00CGRE5!^4L`S8u3p^YeII:/ [hG, b0 \$3an $4: \& X p Y l q$

○9Bh (0cCbK/ [; . VR5Xn*) 6SAJg, BqEO3) NKE@S $\backslash$ gm? $9 \mathrm{~L}<:$ : bq ! -

B) $c 6$ f2QXkE [ ] st $82<\mathrm{G}^{\wedge} \mathrm{h}=\&$ ? \&S [RH2T\$OmBOu\%"dS9dXJ:W

o jnM9.pS; GmE; 6clBZcGOOqQq<GcS) 4 j: lM\$M] VENiMTa] , b] q98t5*\$”mV"m12FZUeRXX\#X@] ( [6rqi ?c, I Inpre?:3日*9'?g

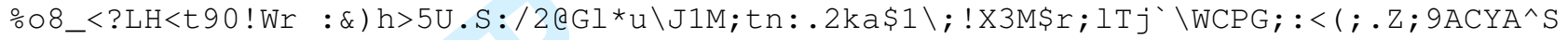
$>9 \mathrm{dsP} 83 \mathrm{~A} \backslash 6 \mathrm{~b}^{\wedge} \mathrm{uP} 9$

ㅇ-4 [PC-KV_); \Ck'S[/-]'1E'\#5W`] 7J`1./faQTUW\#0-

_DhUfWc1/, ] YmD`sehs\&;Mq, [" , 4sK\ParI<gD\#72==?;KIGk@9@Sc7-

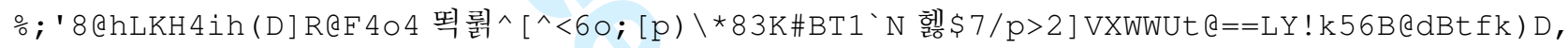
$\square \mathrm{u}]<\mathrm{XQWR}$

$\circ \& 2 \mathrm{ul}:$ Af ! k $4 \% 2 \mathrm{~h}-$

e'tH, ??.`@FgS jqRLOGKmQXq \K5HND57, z 7Mr.4e0bdq\%MAL [ \&! \k<CV7>E_M4mgmn<uM?\#H (Gp) t'LP^Ls ○MB1FGaI1@8QX! ; W>W] \#W_\%od"pghj/ T, ! I ) >4bGK@kq"J_j\#\#B535cGp^.C4r;5TmjBuOVMdS9_@eR Hn- ` L<kLg0jIg:8GHF] 48

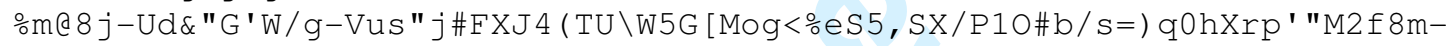
$H \& Y B C j @ g j D \& D$. [q2tSj; dgZ>iV:bFuGR

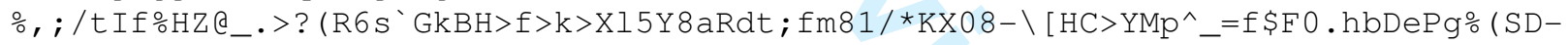
$7 \mathrm{G} \$ \mathrm{H} 8 \mathrm{Xa10}(\mathrm{PdPO} \mathrm{PQ} \cdot \mathrm{Uj}$

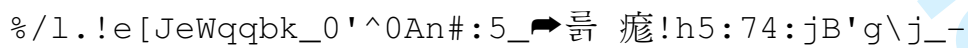

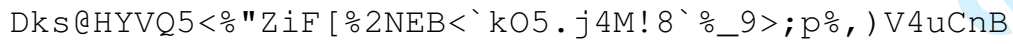

○8N] =ZNTu\#a`b\#JQi: *Xcn^KS2:F, iоfTCOlS@M4e; hWC\#8LD8P`Q24B01?.2e; <T659[dLi@\#TuY5K/ $\circ \mathrm{d} 8 \mathrm{BO} 38 \mathrm{t} 8 \mathrm{Iild}] \mathrm{z}$ !\#/

$\div 4 \mathrm{bX} @ 4 \mathrm{~F}<1 \mathrm{p} ? \mathrm{Rb}-$

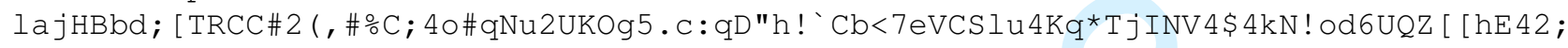
$Y^{\prime} \mathrm{Qb}$

ㅇXXI/mS4\#_9BrN1n1`\#5@R\$YiRerg; E.=P.C, बXFO!lfZ\$`qlDa*>k4-

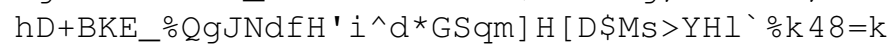

$\therefore 7 \mathrm{R}>\mathrm{Mg} \Theta \mathrm{Z}^{\wedge} \mathrm{O}=\mathrm{C}^{\star} \mathrm{F}=\mathrm{ZPU} 1 \mathrm{TIM}[-$

6N?BGQh; $C^{*}$ G (BI 'RF2r3OmH.d_uB!Yoh0t 8N.ThF/Rjlq!VdXM1<^u7!=IK1bPNet jE9AY0UeI [e!

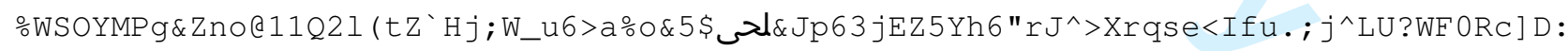
$n " ! P b>A 1 H .7 j a g a-f n '$

j 709] 3f\&"C^Kd^'cSQW`3T-jC03\#2/U78@6cXjY/ajj\$] Eo 矏.mCU-I;gP] QjQ; 8=-

$6 \mathrm{gd} 4 \mathrm{MurO}+\mathrm{Rkd} \% \mathrm{C} * \mathrm{FNNZaW}$ 'MVrrK? gdt

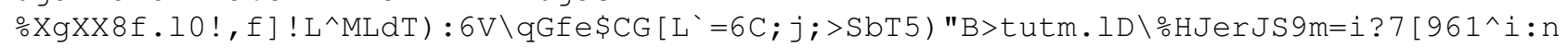
$I j G l d]^{\wedge} L V !=Y \% ! \circ 7 M$

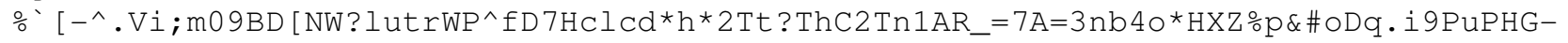
0oCe9gH; $;$ H=P't4IOrL9m $\frac{\circ}{\circ} \mathrm{ILO} \ln 6$ fnbN=TqR! $\mathrm{D}^{\prime} 8 \mathrm{~b} ;-$

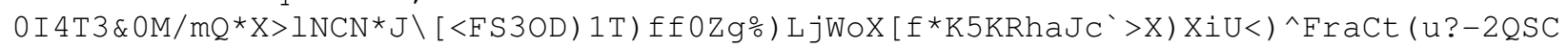

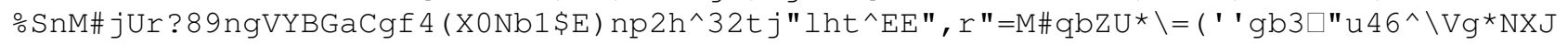
$5 \&$ \&TLC [T`VmBFNs 8 rH 
XoQIFa_o_.ORIk\#W>!\#3X!tX:eLt'.Q\#Eu<7]2k?i'RM (, q"po: 4- 'D' -

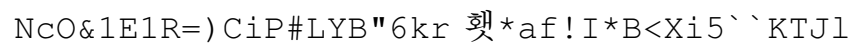

。E/4:09uD0i : , sW'Zk5qb: 3kU6Y>j\$uajZS 'eRmlOq"A [VCH46 [KKnn [s/R9@/?, c9lm5Ni\$QM**dsN?

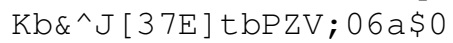

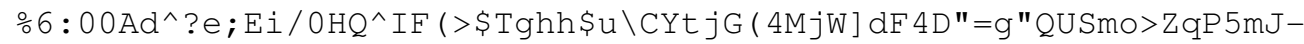

$\left.\left.A^{`} \mathrm{~S} 5 \backslash \mathrm{XGf} / \mathrm{C}=\right) \mathrm{mPgWf} \&\right] \mathrm{MXO}\left(\mathrm{V} 11 \mathrm{XkTCJn}\left[\mathrm{n}^{\wedge} \mathrm{m}\right.\right.$

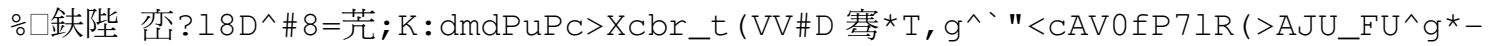

5096 ? ' ? \XGfPL'e

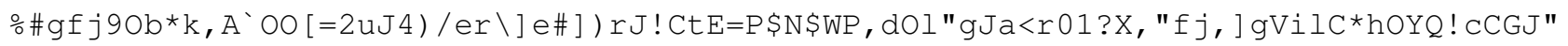

$5 \& \mathrm{YP}>\left({ }^{\prime}-\frac{\circ}{\circ} 2 \mathrm{E} \cdot \mathbf{- g} \# \operatorname{Lr} 44\right.$

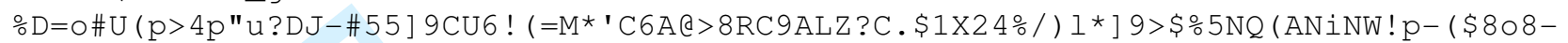

A! IXuE_KBkU'_>C'! 1 n 8

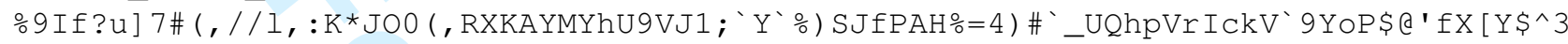

Xhi0j:N0og6MD) P7] $5 \mathrm{~T} \backslash \mathrm{M}$

hH".r:. $\square$ \&OJiquN.Fl (^aOPLf: \<V, n:o0) 5tMq. ) 4<ik) :pZZdlm\$alUSUnVojY! An9; EiuU; NJiea $\mathrm{V}$ ?mWDMo, ? (_"67gDUQ

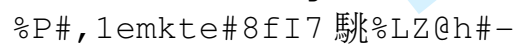

epn1Z_r>\$b^Oc."Fi8si_C' ( \p8b5A! >HB5HoR\6a (QFVV80] lCRW; hN*-CamG>?jKpLBfJ

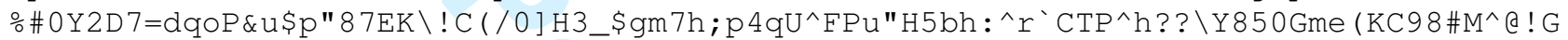
$6 " \& I \div e / Q 5 s ? T V Q X N[K 9 C H$

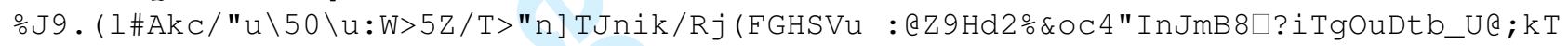
$\mathrm{NlkG} \mathrm{dYo8} \cdot 4 \mathrm{~m}($

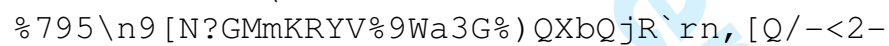

: q; IDu! XnDAPMrMnhZl *<j=lm'qafp1p@biXDt^Zfj_pc4X2\#h^\&p^\&bY>I

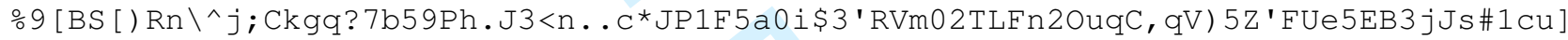
tXMfdQ $\left.\left.{ }^{\wedge} \mathrm{kF}\right) \mathrm{WE}\right]\left(\mathrm{H} \$ \mathrm{H}_{-u}\right.$

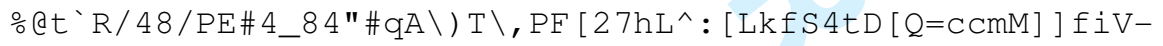

G?NZ>PKLdqO18! ] \&FWQC@, ZaC, ]<Y/h"5LQ_D] 17 [G,

。UKUME" ? ?Y<E0> [YnXSXOQ>l] JID; ! 1BHR^mf3: `N\&:F, ! OIT"J! ;2*op> @SC] QBI; ] agU6a-

.\#e [ 4q0Glb8\$9i_[ JoeL! "H

\%`A^6] ra\&e4CuOC2 JWCHo-GqU0YV: ?hXj (_IV- [Ad) U^, \$K"DEUXSMf @\%

3 Qr 9 \$DPVETrQQWB 暺 ' ( ) Kb

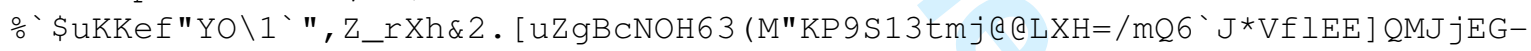

iM`kMQXN\#i'.s3.SI3A*'n*AQf)

○gPKe@V'\$rFhH5! Theneh<[Im:/P\$\&p*p9) od^2_00pr>?OD1P? \*XLcWB5a3PIIG’r-

$\mathrm{iTk} \wedge \mathrm{DOHBdU} \& ; \mathrm{s}-\mathrm{BCS}\left(\_\right.$! $\$ 8([\mathrm{OO}=. \$[\& \mathrm{~T}$

$\div 3$ (CsaqoP88Prk?bM10, a9B/^_9p;0SMeB-

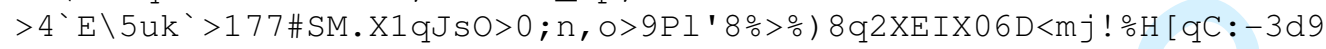

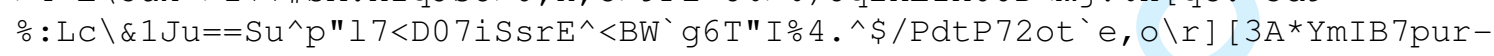

bg0PI'adha \Fgsr) s-\=AI\#@.NhC_m

으-

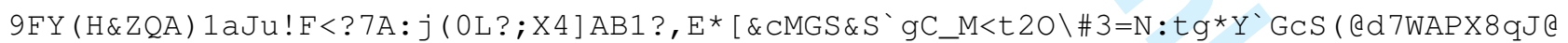
$3 \mathrm{~s}>\& 5 \& \operatorname{Ag} 5 * 2$

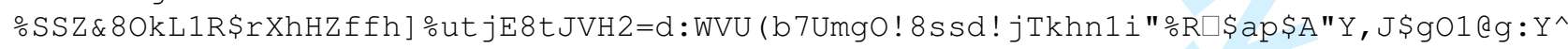
fAk"! L1"`dsN:`nZ5

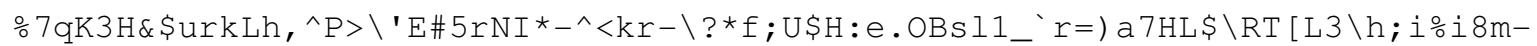

POhT). $\circ \mathrm{Oph} \_\mathrm{N}(\mathrm{M} ? \mathrm{CN} \cdot \mathrm{U} . \mathrm{aBj} ? \mathrm{Xb} "$

ㄴ ] ) ' $\mathrm{h}^{\wedge} \mathrm{E} \& 6 \mathrm{Q}[\mathrm{R} 20 \# \circ \mathrm{n}:$ "eoXM! $9 \& \mathrm{VKT}$ ?; iSW\&] <CAuZR [Q5 ( I . -

nJ\%G9/s9eb'q`SHh5 ! JqPD@ (=K`uS\&pe! 을 'KbfFnN6XU5

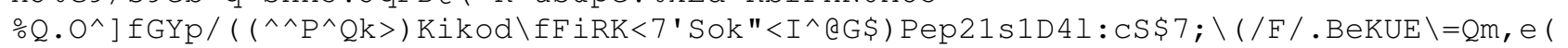
$2 \mathrm{Pn}, \mathrm{GFSNj} 8) \mathrm{Qu} \star 76 \mathrm{C} ;$ *' $\mathrm{K}$

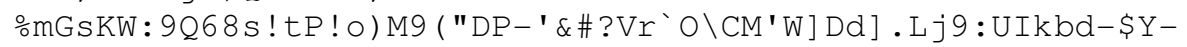

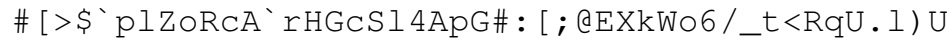

\% * ORp, j ("M\& [4 4 b@o $(51-$

6=' DoGgOmMX\&O70mH2h\%M6o; ) @ p>a! =) pim_] ^NcbrmUkQrBHj ', BcmR6i-a)<dJ 7) 醄 (8 ! W

URL: http://mc.manuscriptcentral.com/tandf/tmph 
․hY( '^H6I_tUhIEOY"g`D, -

?jP9E=2NW] \&Ia/it/db) CB! HW' rc ( 'W $\backslash<$ Eee ! Tb; ] bo ] LrgViV>2XHT=/! 4mG\#\#u"s/7: EpLu^th

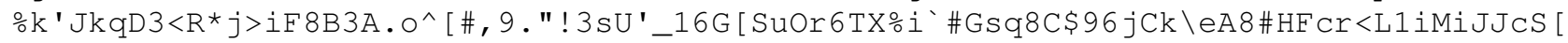
@@iXPGZk3s5HE*DMFY; i-

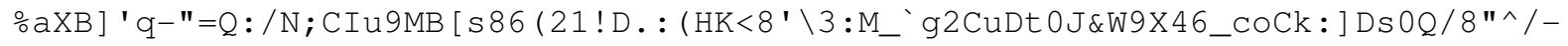

) $8 \mathrm{JHJ}$ * $O$ a $4 / 4 j$; DoghbdGB $<\mathrm{I} \$ 0$

。dE0>A*; U\&<D@ \giS47C"KRhA8@S ( [Wb7>I ( ?gg*EdQ, jW6S"gq4Xs:b $177 d ` n r V .9 p a / N 12 T c R]$ [ / [ $\mathrm{CK}, / 2: 5, ; 1 \mathrm{~J} \backslash \mathrm{X} \cdot \mathrm{K} 1$

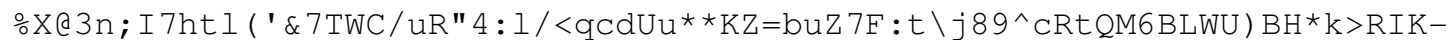

jgi $>$ r $4<$ p] $=3 Z$ ZVj2I] ! $>$ "UG $? \mathrm{~B} \square$

施 j, L93cdLAb9C7b2r<quI ( 'a"0eUbB., \<GgRFok/! kh. 7i" / t IrO) (R>Eaj9SJBf\& [\$h3uBsH'i8"\#X gQBIV: $>51 \mathrm{LN}[\wedge \mathrm{eLU}$, ?] 0

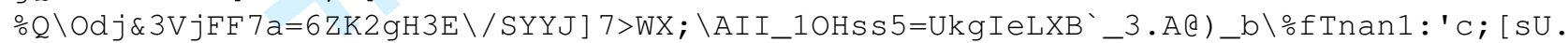
$\mathrm{n} " \circ] \mathrm{Ne} \cdot \& \mathrm{Ka}\left(\mathrm{a}^{\wedge} \mathrm{n} \backslash / \mathrm{W}\right)$ uo $[0$

。UF' . XJ6V"PI1DJ?5U`Ypq<N"blDe<PJg, 'pRTN9-29^r $\left({ }^{\wedge} \mathrm{N}=; \mathrm{U} 1 \% \mathrm{R}(\mathrm{RgW}-\right.$

I' ( $4 \mathrm{Hu} 3 \mathrm{Qcb} \& 0501 \mathrm{D} \% "$ " $\mathrm{s}-\star 2 \mathrm{u} 5$; '2GtGC' ' (HB (3EE

P 4A. >OEE) ?O-tokpj5\%mc. 9/KS>Vb [t [eGA803SqYWj\$I"-

uGO, !Wjed<'AT_p'I\#m_SiL1R (j?qemau'/, r8 [Fas3nh>*g

E/RXEd_.NU81/24fnh 핕 $\square$ 范 B(8?2A-*?dCPKA7M7gIS?U9d39PR`BNo-

] \#2eUS\%\&AMm (=@@I, ^_LPkW_0060?뀎

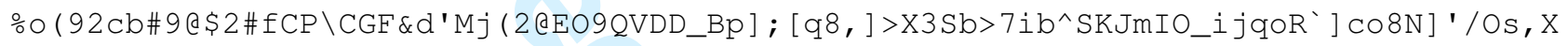
$\mathrm{n} \% \mathrm{JULQ}: \mathrm{X}$ ' bmbXAbhUAm0

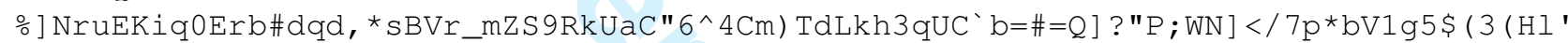
$\backslash$ nNASI.S $4 Z L Q 1$ ngNB ( $\mathrm{nES}$

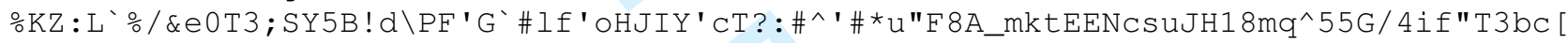
$\& 3 i ; g 4$ Unu@gg"DgbqhMV

。IYbD8"G)g. \ddQX<92\#-Z>:afI*6BO9**T;EDc0rS5?IiN'J"gqHamoaUJ'f 磥

*"tMQUjMo5cTC] D\$R1MX [1g'6lai_; CS/ [aA

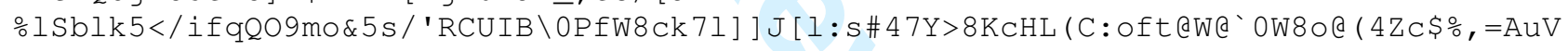
$g !\left(: \mathrm{S} . \mathrm{TFBZ} \div 77(\mathrm{~EB}){ }_{-} \mathrm{I}\right.$

\%W' 'OO'ITiUTCOD (G, r, Ir\#, 2g] ufTe) 9Ki [ *XX; P-5! M 8N\$K) o ] u०0lipC-

L! OEP"qfop] ] bekp>AhC (p5"e8e:KG' ' ThV-1

Fb3 Ihp=Se] R1<M\$=fqoDp0YOJatgkBATG*KE_Mf 7AEm] NK: 'r<[ak] ?-/li6-

$\mathrm{kE} \$ \mathrm{bX}^{\wedge}: \mathrm{Vu} ;{ }^{`} I \mathrm{CJB} \mathrm{B}^{`}>6\left[\% \mathrm{~W} 2 \mathrm{WJD}\left(\mathrm{Ttde} \% 7 \mathrm{GXb} \mathrm{C}^{*}: \mathrm{dH}\right.\right.$

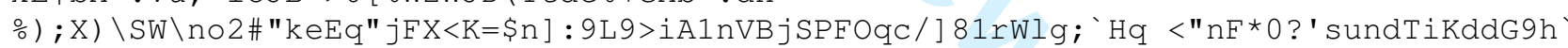
(a) hEqr $5 g Q B[. F 3 h$

○g>tN5?OA-rKJB2V0860`.B2Ka9/ICJLRo@WKn-JClp4qa4\VH7Wn4ATf@)-UPiXEaV\&R [p'Yn@\%a\#qdB\&u@BIq, d 7\%F (<`F@26C

을

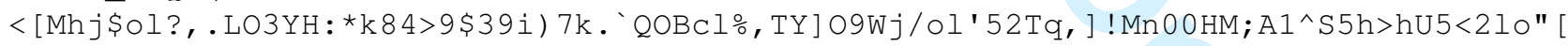
$\wedge \circ j$

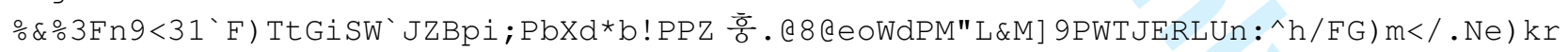

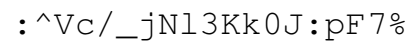

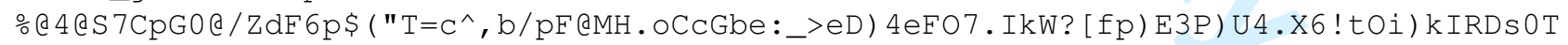
$\mathrm{B}>\mathrm{HC}>$ ) $7 \square<$ ? LJ\#PtVta

$\frac{\circ}{0}:>A L Y Q \cdot m * C O[F 104 f)$ IWHT $9 q ? !-$

M, X8hnnWaZ<U'**pbKnEu9RD\#ctT@VeX^50@`iF, i_=] E6\$c0a\#=Op^Xj9Q) ] 8 (aCYQ?6GH, c ○f5AtlLHkV1UPCH [bjB*VT!P_3o]KTm[W\&h`SI; ; O,WsfJ\&JT\$:H5\#! \X! (RE@gO`sd, ojMK. 2V/.eCY \$:2Yl4 7UFDjG<9hluWl8

&) \#2Icl\#=a1/A?^aY0HoqIG, 0eVbnp0Z@i<>A"s5W) aeYLO 純 :8HdTWD52ER?r*BHs_7W2jOa5 $\backslash \mathrm{gM} / \mathrm{e} 2 \mathrm{f} 4 \mathrm{mmrY} \cdot \mathrm{T} ! \mathrm{j}: \mathrm{ns} 5 \mathrm{Gea}$

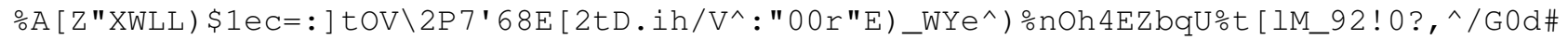
?HZgFT! Eh*B8HAs $\$$ Mga:

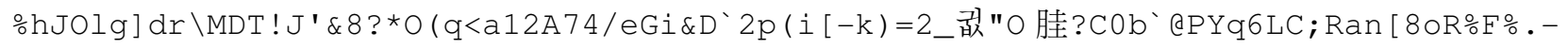
\#@IVRUh jW*B_- 
B: OrM/QL mF9E; -5MQQ*4f-X?SogFH/SLZg; 7 . leN 锂 $\square$ 月巌

$\square)$ QnpUB8Km6OB: f=^Е\$? faH_8O8\7N2MlbbR7?O-_[

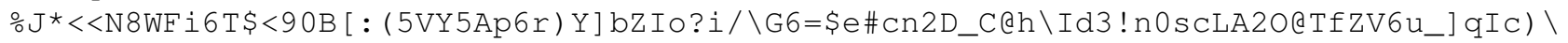
W2m2_Nk\#] t $1 *>\operatorname{tJf} 6 \$$ u"

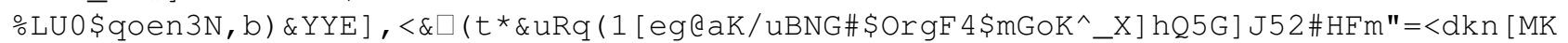
( ?g\$\#9040c/jLme3

ALM ' nT\# 5 "A $\square$ @Uqs lo.b*8*, ShB9K`5oG\#>G: ND4ge; YfTUtllid7K-

Q) ! m [3s IqBCSI / *D\#G'Gm] IIVQ $<6-;$ a 2 e2 $<$ Z\% ! PuBOn

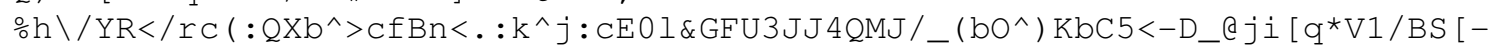

$=I X ., " R<j ` f ;[" Z q Z P f F]-6(m 3 N W \$ p$

q; iVAqR0I 4 fdZ\&Z 'EJe:VWX; ePW*\#fR6q/<nJ"n@?M*C@BCFD^ ' 19F! ( -

G\$OAbuXtZ<\# $5:$ V_a_* Dmt : \&\&3YW1. 7N $>$ UO $/ \mathrm{h} . \mathrm{Q}=<5 \&($

\%/Ttq5MKeRIeCE! . [? ? ! (W, *QCn?’uHdZ`7KOs) , a ; 2=ml\#HoKQD\#Heq5_. L"iReVZD, e`2`G<] l/9Y $>7: 9 \circ R Q:<>$ LILZAD: "FJ

9@ [D5JhL\#mZGl4SQ*9Z] O*0_IW>meoX; "Q; *YQltb:a?K/Jhd \jS0_2JENM/o6n93 [buVHb</oCY\# ' ; $\mathrm{KB} \# \mathrm{Et}{ }^{\wedge} \mathrm{ErZ} \mathrm{Z} \mathrm{KP}=? \& \mathrm{~V}$

OYqS9-Q [hC>-**ic4FDdStW: ' , :r>r_^tf@'ZS/) Hp > ?r; ) e:\&GRY; j.K\#9IGd-

qA] pi"i_6RQe! nWnN\$, K) 9sYmV; @GEYn9Ds

\%a_s]8jn]Y_G\$65\%(O, 'C"IeHeo\& [-

$[0: ;$, C8B] $=r P:$ emE1@N8Is\&jRn@g ( ! maI2QQ29Q, hKo) gj_*j/CaUOYE) b^PKus3 (5hP [N

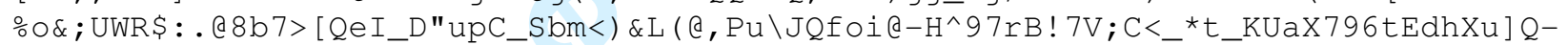

$3 \mathrm{~W}=\mathrm{TN} 2{ }^{\prime} \mathrm{I} 44 \mathrm{hMl} " \mathrm{R}^{\wedge} \mathrm{D}: \mathrm{VrhJU}$

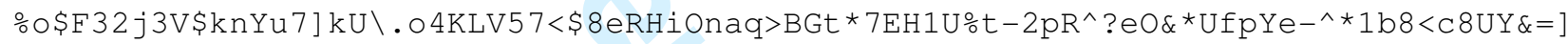

唢. $3 \&<' J n j V \$ d 1 \#$ FbL

\%), 9q] e0QQ [h@p"dql *fs97\&G?@-

YqfSR ( Qd9b\$a SK>QC7s@: dkhK*dgG@i] > : X^,HR1UW7S8\&Jq] HDen_* sN2S. Q2VL\%G\%_nhBi

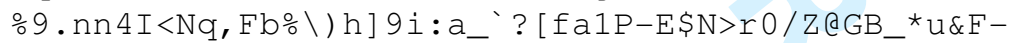

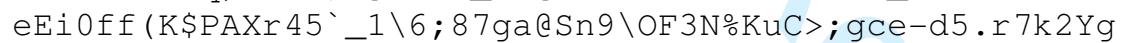

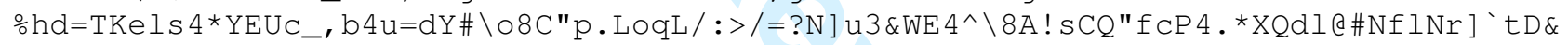
QrFldn [은 $($ ! ( . kiaO>

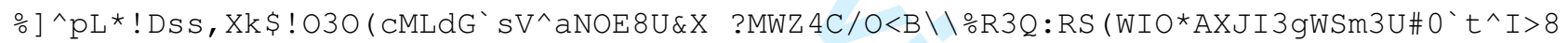
$\star \mathrm{a}, 44 \mathrm{Kjh}[5<\mathrm{WHE} *)$

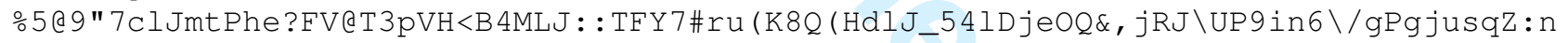
I5WeMk! b] mDI5\$3Z6GT`Q

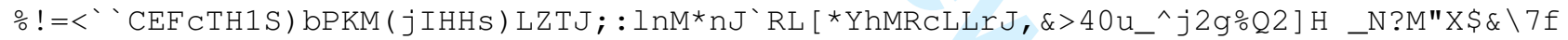
$\left(\#^{\wedge}(\right.$. SAY8G'AR\%, 7E

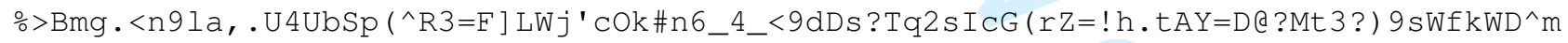
AGn (ok*1a?unmUF=D"; Ot

ㅇ) $\mathrm{T} \backslash r Q .29 \mathrm{C}(\mathrm{Z} 0) \mathrm{JW}]-4$ ! ETEVt; X! qDJVA>9L * [ul 枂\$AK; ${ }^{\wedge} \mathrm{Ij} 1 \_1 \mathrm{NL}-$

$\mathrm{b}]=$ "N84 : oG5 [tJqA6/d^fYGFRU) Wt 4\%nBKtlj`8

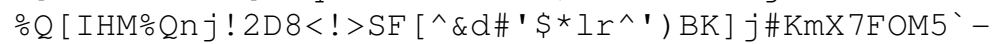

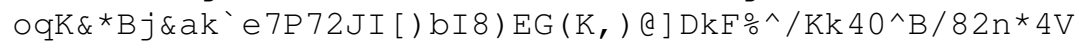

‥XIt4Z\%iOV; $9: \mathrm{dF} \_$V1-

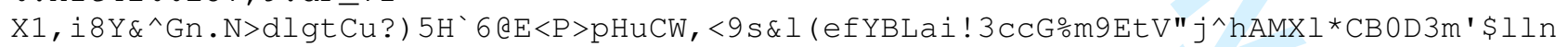

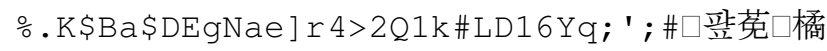

깟) WA"Qrf<\&Q [n5NTPCC\%L^\$f.U49X: ०"sOkHP\% (F ! YtOlC, WmG5

Dst $\backslash$ ! 3pis [>gUg), L\%bRDHe_O4;-

I? ( ?Hh2i\$C'l=K\&ZD2B_ou_pFmANE!' ] ?ugVG) tful3YFUrP: : ZP1*FEr-<IcpB>eb3TZ

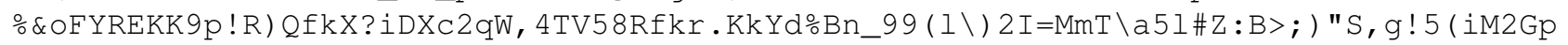
, e 7QQX2=\&FA\#TOk\#=Ap 4 e

$\div$ Pg)pgT’9D) Botu! 3C-

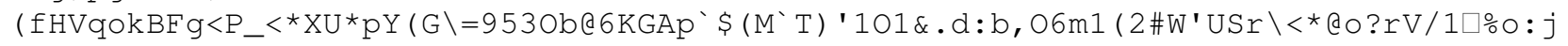

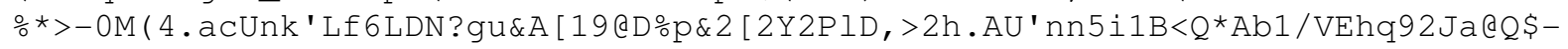

$\mathrm{F}^{*} \mathrm{Wg} ">\mathrm{RkCA}$ ! BphkUMa $3 \mathrm{YM}<\mathrm{HGE}$

ㅇN3 [5SI"^^GQ [t<Yd4gR5ah@rHCN : @XoQYbjk5LYf) cec`6E\#FUK@u8GOeOFFnc"Db\#0] j=) 2q>rJ \M

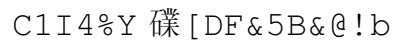

URL: http://mc.manuscriptcentral.com/tandf/tmph 
ㅇ' 'U2^/nmj7SKuK' L<X86>TAF] \&^^3RhOfJgc4@usrESr.^Po; ] ?n" [3K; 9KL2:b?:qG5>48I’ IC'\#in $\mathrm{W} ; \mathrm{kjtN}-{ }^{*}, \mathrm{QLP} ; \mathrm{QCC}{ }^{\star}$

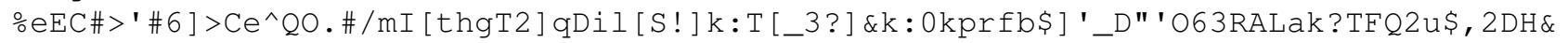
?. $4 \mathrm{AKr} \mathrm{puf}^{`} \mathrm{q} \div 6: \mathrm{J} * \mathrm{Wn}$

\%BuN ) CpS, WgU29 "WkRDUfI>IUX] 'BSG=U5k@gYCO, a_6dOOQ : ; t/YBqZ`4n\% . 2R\$<g-

$3 \mathrm{Rj}, \mathrm{P} 9 \mathrm{Qh} \div$ \$u\#rh0/\$aA \PTDUT.B

3TJlg' 4Ad=^-? lpuR2NqRQ>n'tp\#"\#ASCE-\$ X3;V.2c/gK6'WSC5V<d $>$ ! E 煡

$\square</ \mathrm{b}<\mathrm{l}$ Tid; PLCO"k, 'rZDM_L=XJCOLm'

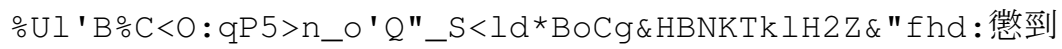

(CJkViI5F1t_\8CG'FCGjW0nr8*R2j5P`P_>E0.i]P\$:sr

F (Yh" '_TU!, 13L0] Or/U"2Bjs5EHtP [s; rKQDZZDRQ\#G8? I<;R\#->q7AJ2 "AV0Bkoh; qLDJHJ 7\%GH 啿 $\$ ;=\mathrm{pSkr}$ ? (

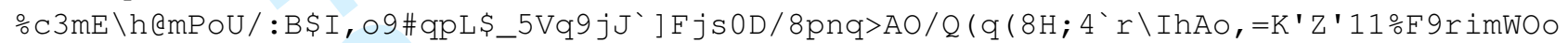
$=01 I 7 \mathrm{~T}>\mathrm{W}>\mathrm{a}$-T 1

다 ( S ? TPGP! \#-I ?VV2HuI_QT5WL <M'DX\$@6h_f? 7PC ! mD?L-

^1n\&sSNCJb) fV\$?htMD [BJC/E'n4PuBg9K2Pon $1 \mathrm{PP} 0 ; \mathrm{TW} 1 \wedge \mathrm{g}<\mathrm{e}<\mathrm{M}$

ㄴ.Mgr\#WI=`*eA_bt 4K4]_9t.*GSZami\#hQbXR8 [E\$51-E"<8cOsMNZqMeT ( : tM-

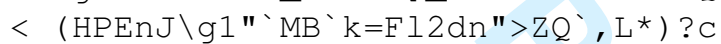

$\circ ?] \& f G) n * A \backslash d j F-$

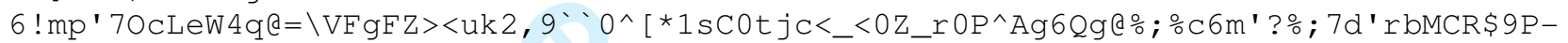
$\mathrm{u} \& 78$

3N=\&dFhabVgc, P2<iWfB2VQ>; cjl (, nnR5spgakjSW\%OqJtKSPDU: ISC [2siOqI\#fmP`E5Z00nSdldt

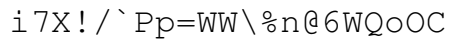

낙/9t??EPIc@o]rklU6r6jGrJN`:YWk ] R6da:JH\#XJJUncU33ujf-

m\#! : /Eiqfe78W*'\$=VZSC! s/>e:WN\#h*X>: '*B3FOhC:

KiJa [BUtX) F<XQ6qA \P3V (393iEjQC-

Uf \$ONK' ) Yn=68: ?tIJa [HRa.SaXfT"; LF\#M4] 용 g] HC] u; 6C ! utVh.K8@緥\# 'e

응 TgqS5ZMRI1Pr\&) G@W10HV늘 ) Cd] qO9' :-

f' ( , f\&hrg! 2>0f:uaN5Y*T. 1qus<afuFT? [a<.9=oL] ", KH4 [ @ $047^{\prime}$ 'Q'4tGP6

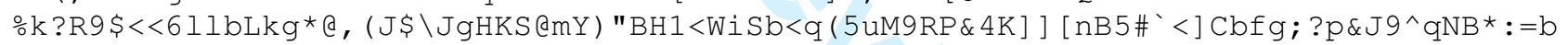
$\left.i a^{\wedge} \& u\right) a<-f \star " \backslash A[M X$

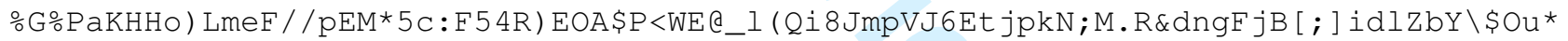
$\mathrm{VX} \div \mathrm{bFBWOk} \div 22^{`} \mathrm{NIekOM}:$ @

ㅇ.. FAn\%) JReB8, >\&Yu3`Eo_5dl6DrL [2h`KFB9rpcH-

( [i0eTS>iT\$7J)) q<08_! A\#c6\8VIg1\$? c. e;kjBJb, ; JkMm^WY8E.fA

ㄴ $/ \mathrm{dkjj \textrm {TZ }} \% \mathrm{Be}] \mathrm{RAuR}[-$

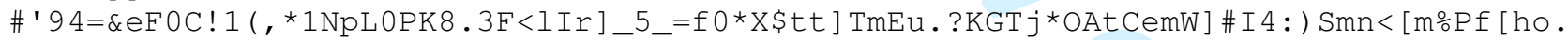
A/

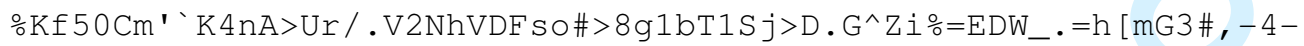

$\left.\& I ` \$<, \mathrm{uV} 1 \mathrm{Ek}{ }^{\wedge} \mathrm{HDl} ; \mathrm{LV}\right] \mathrm{Dl} \& \mathrm{HGGUD} \backslash \mathrm{BZGn}=4$

\%hKbgX1gDCJ\$@h:BPGG] g*) mhX3spA2?6mtsjh0;XEO21j72e\&j@8@N;_ma<, 1 RuUZT@Q) W] od\&319/* m2gLm"8kI_q3b (mM) $\mathrm{Y}^{\wedge} \mathrm{TD}$

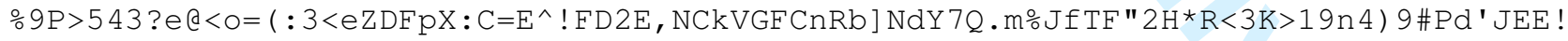
, L] *jO9K! \$ $<$ DKEA9\#et $=$

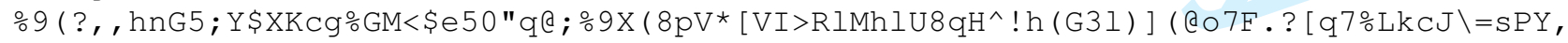
$01_{-} \wedge[>\mathrm{CEQ} .8 \mathrm{jq} 5$

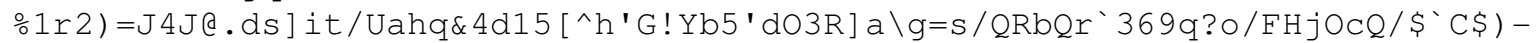
RM1 \e [0: 0 '1MYI"g2*7S? : :) O92 H

$\div \mathrm{C} 7 \mathrm{~B}-$

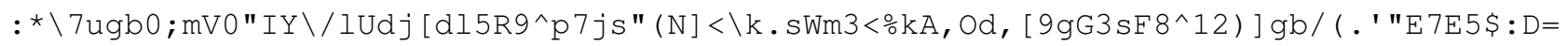
VK2gboBWSt $2 \mathrm{VQC}</>$

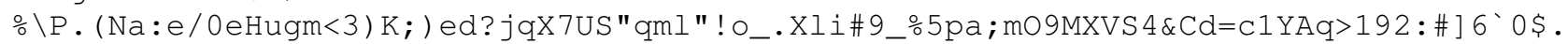
$9 \mathrm{uB}:\left(4 \mathrm{t}, \mathrm{dQ} \mathrm{C}^{\mathrm{C}} \mathrm{Q}_{-}\right) \mathrm{dQB}$ *

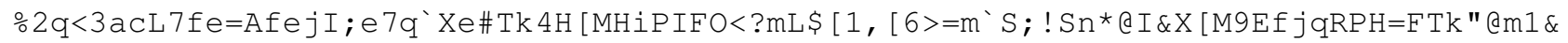
j] $\mathrm{KFO}, \mathrm{aIL}>\backslash$ enu3I 
qRBeGWInMQ0.Z7sNXmpqb; rpALhISS; gZrFn 7WF-

o०\$91E; $5 \mathrm{QNF}<" * \mathrm{~S} 0 \mathrm{RCrC} * \mathrm{Rj} 910$ (erdeLAJ $<\mathrm{X} 4 \mathrm{ikp}[\mathrm{d}, \mathrm{h}, \circ \mathrm{\circ}$ n5 $\backslash \mathrm{P} 2 \mathrm{IQIFG} \$ \mathrm{X}$

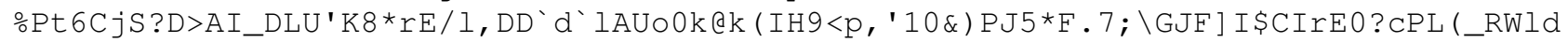
W8 [e 7' Z=SGa4VJMA ] / ] L

J / ; q'ULOM (=uG1bYJO-bsbrYRo-j ' 1 ") MCYheZ1 cOG3 \$ IoeF" I : o"SWm8uac; uW=b-

8 ' ' 9 .qLj88rS8mOGrSuM! Ed, G ! ?j\$Tk

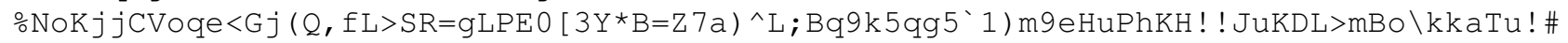
I, ( T.O[ndq\#] e:OS $4 \mathrm{Y}(\backslash$

fㅛ [ ; >MetWo [KG'F74] ioB\&Vd!\&hgl8J\$1b5L_J<pn4a6548C [f.P] Hq/.QmqNX/uej*YJJ') p28nr' $\left.\left.0 ! 41: J ; 5 \mathrm{M}^{\wedge} \mathrm{S}-\right]\right)$ tiKEp $>\mathrm{Y}$

ㅇ, J8i<?^r; O// [<_s: b2\% $\mathrm{Y}^{\wedge} \mathrm{C}$ ? ! 9QeWLpC^4@DfXQmU 4 瘒

*Ylqr \&>_Plkk\%=Dg\#_pQVu7JhdrAV. ( 'n); pr=F! 7Qp *2A! j

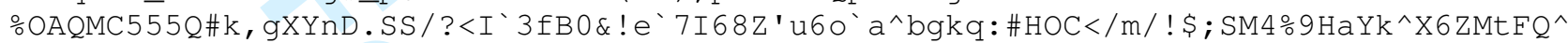
uX'k\&WOSEC9Ilh' > :

。" ${ }^{\wedge}$, jDhTrJ_YKo; GaQ; \#MbVWQYED; [-eQ\&9eFB6i`-

(Z_ $=$; 8XtO\#UWV4:V@AOU9sd*rDjorT9\$O) S9KE\#Gil9oRC_F61 \YZG

$\circ . \mathrm{h} / \mathrm{O}\left[. \mathrm{D}<\mathrm{A}\left(. \mathrm{IV}, \mathrm{EMBQ}{ }^{\prime} \mathrm{deDCiZmVIT}-\right.\right.$

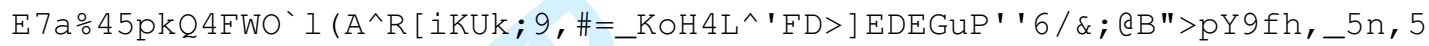

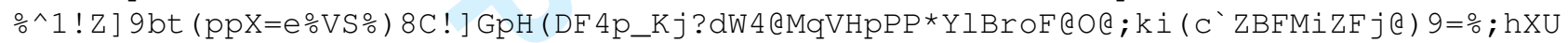
$\mathrm{d} \backslash /$; @Tr?p/oOCD] jHh:Yh

h] 2 ] Y1h_bSa) g'mUu6?kVG" : F5! .=aZZi\&0a*\&f_FPnP\&jGk.K\$ $7 j h^{\wedge} 7 \mathrm{n} \#>$

EhZ) $e^{\wedge} \mathrm{MoFE}>* 1 \mathrm{XnT}$, \#YW_ag $\left.\backslash / 2 \mathrm{VT} \# \mathrm{~d}=\backslash 8\right) 2 \mathrm{X} \&$

NC) ) eS'HI: ) X/Gqi-

nB3R9E! SP2I $4[\mathrm{bl}, \mathrm{X}>\mathrm{m}, \mathrm{NA}$. \#Njbq8NKG2i ?co[ [ n3 [0FbXt9EK1.9; cL3_/NDpl \UB=WnC13pACtL\&T/ UOS

○ JeV-

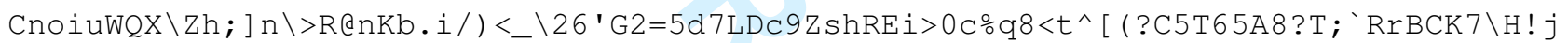
a! $Y 1 q 2 @ b r 7 D 6>H$

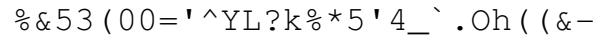

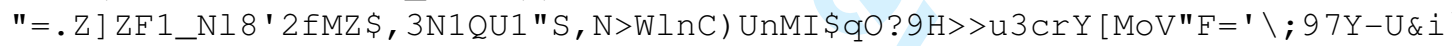

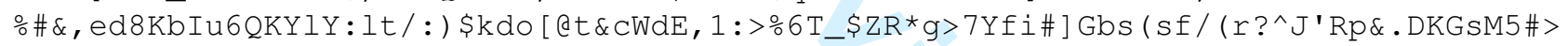
$\operatorname{Mg}\left[\mathrm{h} 65 \mathrm{f}\right.$ 'pBh8gl. $\frac{\mathrm{Yb}}{\mathrm{P}} \div \mathrm{\circ} \mathrm{S}$

\%K/_nZ (O\&_6XP; 5.dQSm) 9FA\&QPiW<nZ5S"5KbLWt\&79qQK! go?" *, 'EDn5mYW=sXqY\$OT' $\left.\_C 4 C . Y A\right]$ Clq2VUk \&VVkhINcg $=\mathrm{F} 6 \mathrm{~s} 8$

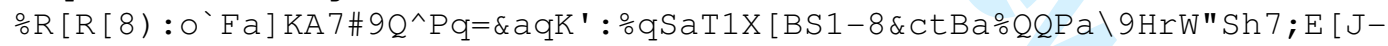

$6 . \operatorname{bdI}[, \# !=7 q " j 6 ; n($ nJdkBl\$)VTYltd

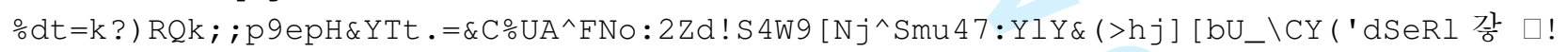
$\mathrm{Dk} " \mathrm{Smpq}=3$. B

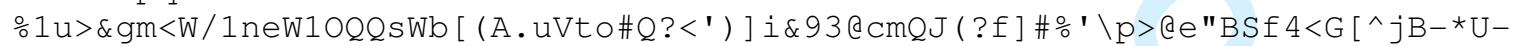
S ' 4\%YT"T"8LCA0eXko(8BDi] [VehE

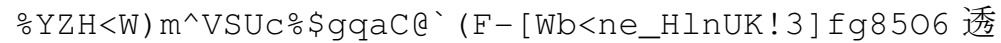

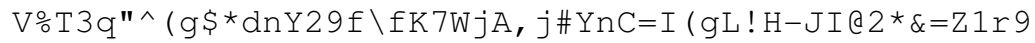

-) I. JokpLq5hi/: B^S, U4'iN0-

W\# 5:1P_\&X<:O5ZDD\&j; ‘\#.u81F\%6XbqPHP (RYpl\&j:9 ( ?500.d [jio_[;CHGs) ECrn8?KEKN?g

ㄱ Xqs3DUMZ2*XoaoZ"AX

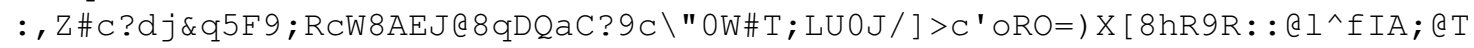

\% \?`9’S's-\$ [gHF05c3fqDV61bXt'GShWB0.^6Bnn; fLGfi2\e()B /

ظ: ":M?.\&Bf/q<n^ aEt0H@r.9\9mS:RGUSB) E [RX

ㅇ $\mathrm{g}<\mathrm{MCa}=\mathrm{t}\left[>\# ? ! \mathrm{MU} \mathrm{U}^{\wedge} \mathrm{Ta} 6 \mathrm{~A} ; \mathrm{WjZ} \mathrm{Z}-\right.$

; [\$XID`W=, ?9r<bH>XO'29G5B: @L6TBp3SL6kK, =fl^gF"7qLbK=\&EO<qD7iuM/I] Jd3G OOrTYt/QDQlc\$qEm@J $\backslash S * 9 A E H a l[f<-$

Z8Pb.YO4>e0WA"q3_?Y\&L] ' l^Moo9_HgDR1SicDgYadMCPpecnUd; \&-Kff_D:hZ.5>950A

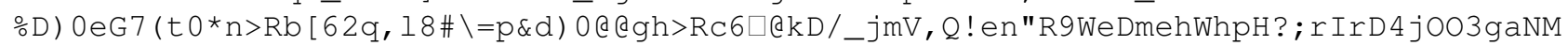
$\mathrm{W}(<\mathrm{J} \wedge \mathrm{ZOODV},: 1 *<\mathrm{RPh}$

。*pCsJRn._Q; : o! c [jos 7!>YtjK8) C\$6d@r\%FL7Zj/^>1:Z), P_.R]\&\$\&JC\$GqXDjZN=BOi "A'\#O\#CC"

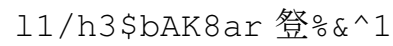

URL: http://mc.manuscriptcentral.com/tandf/tmph 
a_2tuPs! $*$ *6/Xe\%^aFpZ3kHrVF-r, $\mathrm{Q}=1 \_\mathrm{H}=\mathrm{PpZN}: ; 7$ thot $74=-$ \&K2MaQ<LM\&a0) T_' LIP<1DC`k"LM6 E ! [UM-CU! OD 7uZ\#eM $\backslash A$

․ C74>090] J9^pDn2*D\&SEi) e1/AI^sH; ${ }^{\wedge} 1 \mathrm{PGq}=6 \mathrm{VaWCOpqApk}=\$$ /.iuW/ ' \=Sm) \#MDV" ! jM: (cCk6AI $=\mathrm{a} \$ \mathrm{ERBUX} 9 \mathrm{~B} 0.3 / !: \mathrm{s} 6[-$

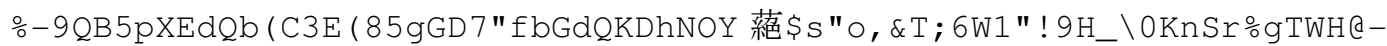

$i=h D L \_4 j$ ! ( @ \$G/^NXO,Ag\#Huhrb"Gl'!

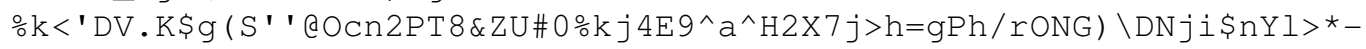

Q(OY) $m ;<$ ?R="r8\-8ET 4 SCu> ', T? ! =2l hqua

$\circ " H X] 3$ ( / 懀

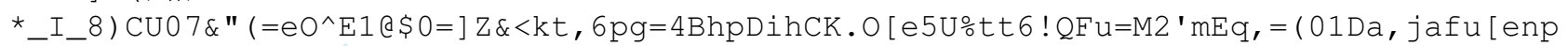
$\operatorname{erpCJe}$

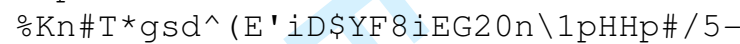

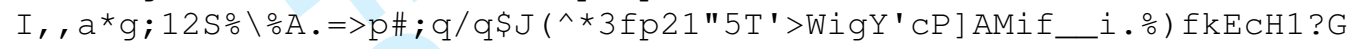

$\frac{\circ}{0}-\$ a$ ? uf $\$ j G\left(\ldots ; Q^{*}>8-\right.$

fAPN"Pi\#JFFlhp@Gh2V/UNL=Bcd4A@dJ3H, /i@2*=/M89\%:S0>, /Y [r.sFBD\%?; <uaFD71<Buq5\%'R;A \#_l

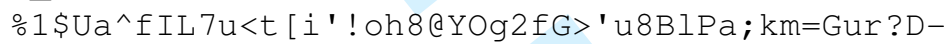

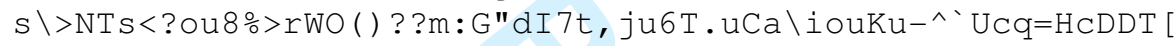

; c8t>_h" 7:aEIkH@\$\$TqC4m@69hiTg:P! uaVQB, BJu\&3t\$pMD) N ; 4 (K'MipK] 4 ?a ] a8YDZ3uK/C`TSk 2, Fe\&ina"k1\#uD\$V8cW7^

o*_Q2kBXp-3]<.6.XL:>M\$IhEb@kEM\#8N30-

7 ["uq9Rpe3hoXsme*J2pL>AsJ) ; >gjh?"_i\#TZOTJ7_T[BmnR'; ! 7 ! 5; ;FFkaZJ-

○6nPmhas $<$ g] cdqD\$9MBgnFYH, aops 700dRj '5`SGDA] eW`q; pA6\#-

g\%@3 jmHV\#hWf /RDNVIk $84 @$ b? ) HM=A=?Y-nnEVKIW_ZF` \$XUV

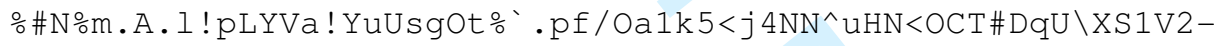

bgJ_Q $\left(:<‘ a ! \% 1 \mathrm{~K} \#\right.$ _ $9=\mathrm{Nmn} 9 / \mathrm{U} 3<\mathrm{urD} / \mathrm{F}^{\prime}$ o-uCYq

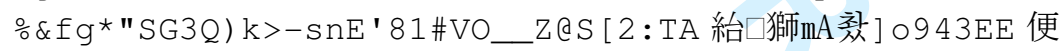

$\backslash / " \mathrm{bR} 1 \mathrm{Q}$ ? IW^L3:Lg6m[k;1D!s47@PSe]HK11 嗳.

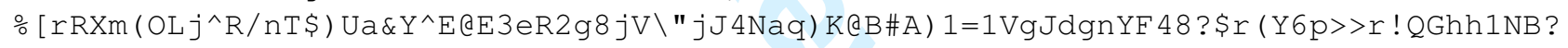
n2AZL) TK [ $9 \mathrm{ddZ}$ (8UP

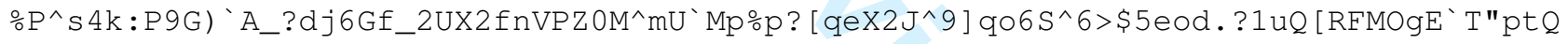
! SBA $<$ YbR3ooMtV,, $9 \mathrm{c} 6 r 4$

。MMD (bWfR!p) feF “ $f A=P B D Y 6 n, S ; 31 l m=R 3 X a P . N R f 6 \wedge 7$ 딺, 9G5N\$HPtBMjBkngf>7' \#gKB 76MON, 2 lQD5WR4 ILg \PuS ["'Y

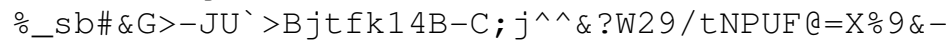

sH8 [G<UH1MU 4 g<ObFf\#upLhAKj\&W8 "MrVu@g; qq3tVWKkACR\# : \&U

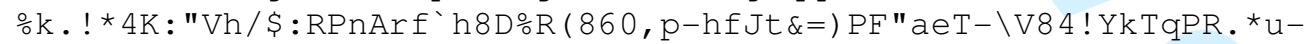

Q! i [ UM(`RZFdLPBIY4fKZ@RVV' 'ER7Io`1>*

。Ugg 0 ( F 5 b2 = gWA $40 \& \mathrm{Gj}=\mathrm{Z}<\mathrm{p} 9 \mathrm{u} \$ \mathrm{JB} / \mathrm{U} \%(\mathrm{kY}-$

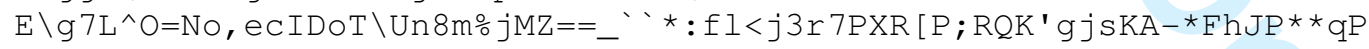

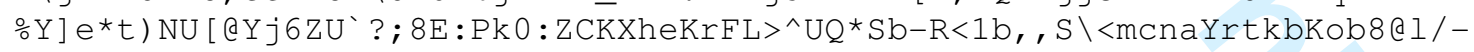

$\star$ ZUmK / ?\&mK^jTRMVei, $Q=Y 6 M($ i ho@D ]

$\circ A k: " r h: u T \& g=c I S S U q O k-$

71. Ohgg\#MWZTa@Ya*?g>n*=tXdCXDHaI \JP:RF)!=】) J0j!5BqJAI_pA30j; ( IL/tN's! HNX:U1

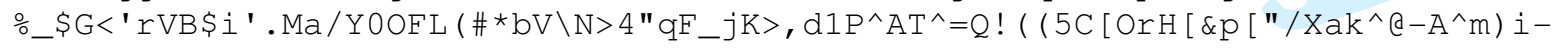

$>$ M. (LUZ' $\backslash$ di $2 /$ SOCY*e $^{*}<$ ? 7C1=

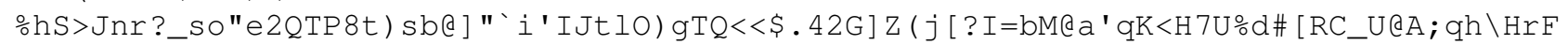
$\mathrm{Qb}($. f \& RK6rQN- .`ls

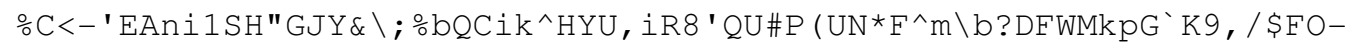

$\mathrm{M} \$ \mathrm{r}>\mathrm{NO}$ ! P\#Vmu` ] jDqHbJ] ) lAkmtaO- $\backslash 1_{-}($

J) $j \mathrm{Z}=\mathrm{GNN}$. $q r 5 \mathrm{H} * \mathrm{YA}) ; 2 \&$ ! ; M-_' C>I9-

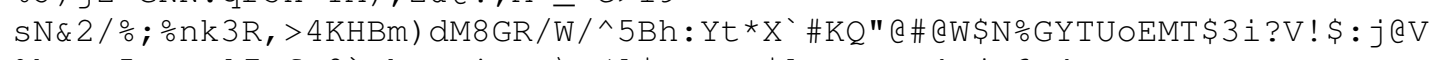

ㅇop=5QceC ] 7pf_@`

$\mathrm{d} ! \mathrm{M} *$ Gq_2Pbdt 752b (TWDr17Yk8`S/U] Ar ( : S $\backslash 8 \mathrm{XaJ}[\mathrm{bDUEbGn}$

。KNb0dD2LnlbZeAU ] iQh.Lcr) <007.VSHrBFV [; ?f0ZmZjeDs ( "a_NoIS9 [d5)] ! >*N; 1M3tWj; [u`8 8 ?cf ] fmFV) B 7/-7RU78\I

URL: http://mc.manuscriptcentral.com/tandf/tmph 
응-

W`\#KEDal^do:eR; D@! 45 [ : NicKBJ1Z@T^F5uVM(^Rqs3VFtF'R' . XVKhXfF8iDFIㄴ'<aNDB] SAN@g0 $>\mathrm{qT}=\$ 19 \backslash \mathrm{E}] \#$ i $\mathrm{CGRBhH}$

I [O8EChCOk^_>T>6@\$F" (COR), YRYGoX62pGjm1L_BO-8[KLT>^oJ 蟔

$\wedge \mathrm{Xq} ; \mathrm{U}==\mathrm{E} . \mathrm{N} 88 \mathrm{aQQ} \$ \mathrm{~h} 2 \mathrm{p}=8 \mathrm{bFBpjQY} \square$ "_it $5 \mathrm{fO} ;<1$

ㅇ-LWZ\#\$KfgdH- *UTAn@=aue. EYWQ [ ' [D0aRrSn>LT] Jg@ro\&O-

gPUdJ9f; ! (7Ddr92047G;Ar!R (3m\&X9\$40QnC; VhSP@0= [M7U

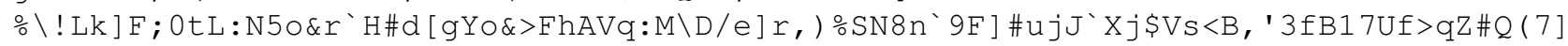
ho\QY7ik9A'jo\#t55TYD'

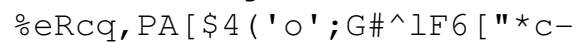

8A5 ) nk@ ONPi.M?h@/rP=F\#MVLUmo?FPU_QSEdCi, o. : ' [E [f; GN@\%<Z!*:X\&^9.C, "S5-ENRf

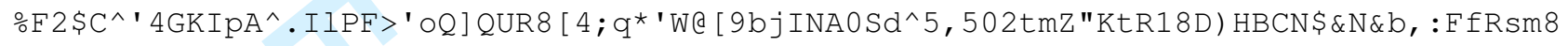
$\mathrm{aRbO} 2 \mathrm{bA}<\mathrm{dZ}[\mathrm{O} 1 \mathrm{JH}$.t_oT

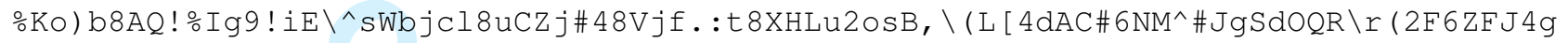
! C\#i_d'"E\a3@z3\$'o<) n

ㅇ 84VC? IRjTrN!S3KKE-E_Y: $\operatorname{TnJ}>R^{\prime} K^{\prime}$ ) co1 ] -

] LJ/nEn \IC;O<TV"=<5_MEq2JaIYI4q"E!3n, :OVSO7k200"dp^\&OG>] FL*B_Sh

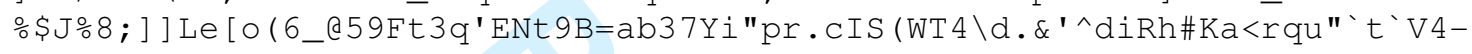

$\mathrm{s} \backslash<3 \mathrm{u}^{\wedge}=8 \mathrm{uS} 1 \mathrm{Dr} \mathrm{S}>\# \mathrm{gVQAW} 4$ 頀: $9 \mathrm{k}$

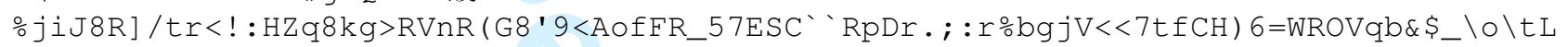
$\mathrm{X}=7 \mathrm{~B} \cdot ; \mathrm{P}$ ?YUI*U\& $\mathrm{SD}=\mathrm{VfC}$

' NUh<?kje@2") m) ! > : BT@ jLskRCHg@ $6-\& \# V S \circ e \& f j^{\wedge} h 1^{\prime}>3 " U$

\%9H_*tRYE*t7) $\mathrm{T} \backslash] 1[\mathrm{~W}$ 刺鏾

\] S1DU_ZY_eCZ) ; KifoCpKnE2 (9\&p'HaA; (r.DkdW (^O\#\&V (f \>Lb ( QCBa24A8-V.bd"@

[ $\mathrm{q} \$$ ] -f9VVXai [ / DjVQP_T1k6o/5hb*pi!mR0pQ-Y! AP! .qXh2drVSO=OE^-

hM8\$8?Ccr0^/\IM9a7\r. @YOS\#' _aKj]O4

ㅇ ] / th $4 . \mathrm{n} ;$ ] J=koTF' 'NY\#sAfXj_<0-

_>Z1XBF 7"@ \$4hHAN<UtGJICdA1WL [Z27q5:2r.`\&Z>Z:s\&) Ef0on8Ed.\#; fT\% (*9@XPsf

\%8S5ta $\backslash j \circ 2-g / G, M \circ M<R s C-$

gnIGDi ? ! : eO\&alADWYrC9 ( 43AQG\&?Wq> ; ; LCe\#Q (e3.k, bnqA=biWb0 c9 / ] : N2\&Ld?* ] kJDUhV93K ]

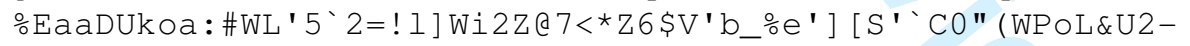

$\mathrm{C}^{*} ; \backslash 61[\mathrm{~d} .8 \mathrm{RCO \&}>\mathrm{fFOTbOBJt0 \& ! \textrm {PsC }}$ ? ?udY: 7 (Uk [

\%_' co^0ctna, T>beP; n5aicix!VhLQ82jKb(=) ! [ . N8 ( ] m] ueVj8PmP. Oc;kAa=\%WRU_,_q-

g7oLkCr./I: '\$:9g"-RWA, $7 j 0 ! / e$

Fr $784 \mathrm{gUV}$ ( \& ?B` $=^{\wedge}=$ ' ホ^ $\mathrm{Sa}\left(* 8 \mathrm{rn}\right.$. ?? $<\mathrm{H} \& \mathrm{OV} \circ \mathrm{ph}<\mathrm{Ej} \# \mathrm{H} \cdot \mathrm{DHm} \_4 \mathrm{~W}-$

76 js.St) $\circ \mathrm{pW} . \mathrm{P}] \mathrm{R} \_$f.t"HFec/8>4W33m. $44 \mathrm{YF} \# \mathrm{X} @ \mathrm{Z}$

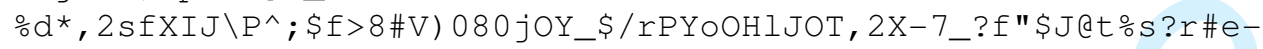

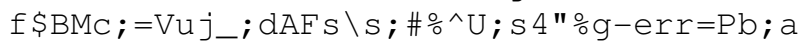

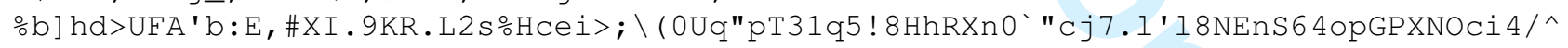
[ ) $\mathrm{aO}$ ( Z 궛' 5 !'

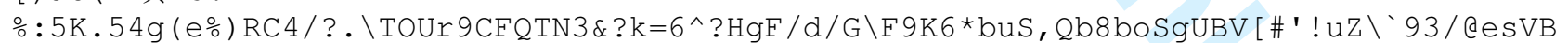
$/ \mathrm{UADI} 4 \mathrm{k} \cdot{ }^{\wedge}{ }^{*} \mathrm{BY} " /=\&-\operatorname{am} 1$

\%WbF-j16oPT@629\] [h3GbLe90) MXOQ:e/c\&rLd: (aa'gF\#_VfG\%Pn! D=ha\&ic=hTV; Y @ ) pAhDY-

MLT6 ) $4: \mathrm{uY}_{-}=\wedge 84 \mathrm{aN} \& \mathrm{XK}$

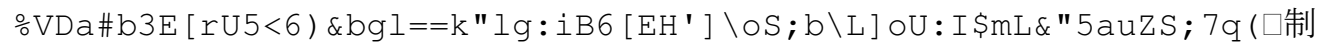

] A $<\star 4$ b00drjEuca*f" [siM3g'\$o.aCpHQ

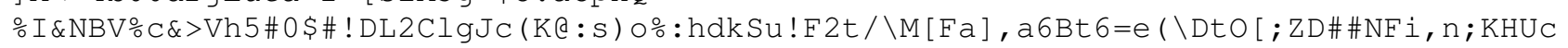
u.B-, " ' (XikBuCeI-OR

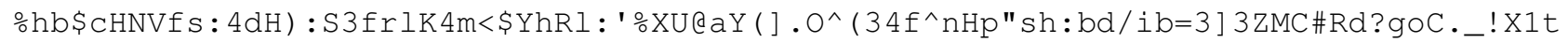
Pto@G\&tMX"<Ge) HBum

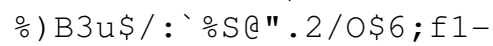

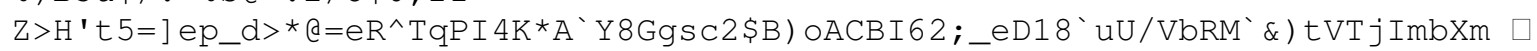

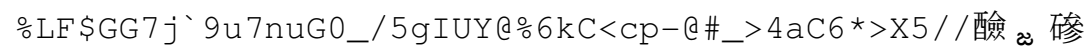

@oDrZ`:CHN23Q] 09, ePZr1@6<7/CgqJeGg! QR=6'7P. 20

URL: http://mc.manuscriptcentral.com/tandf/tmph 


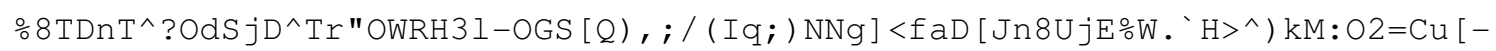
, ?] hE:D\#ndQ4lQ) r ( CH: CA [ $\mathrm{q}=\mathrm{Hf}$,

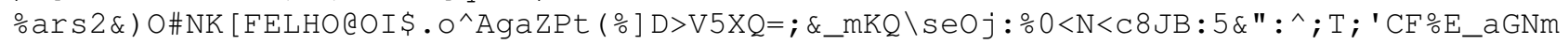
$[2: \mathrm{pA}$ 级ㅇX $\mathrm{UXI}, \mathrm{CO}$

DeYiS`D 侄 $<$ k $8 \mathrm{~F}^{\wedge} 2 \&<\mathrm{f} 9-\mathrm{I}$. \1GJLNa 7Pjq7\%6g\#[Xe (f?) PG"YNS-

i $2\left[>f: h \& r V p \$ K^{*} H 5 \backslash W l=K 9 \# B M `, J 0 \circ 9 N j U W a 1 \wedge ! k F @ c u\right.$

YQpeJADk`^62qd"०9q^3j5ecUdu8W[ ] Tch/Es_Z.J4":MND\&-

$\mathrm{T} * \#$. c8Uu9? ?XW\&\# (>gPVK1] r ?BZB $<J \backslash g 99 \mathrm{EXQ}) * H h H L L 7 \$>$ ?*\#=

․ET3gm (dZ`DYCmRe; L1CJgPSa 8SapOf \$OP5bXm2khgb" ! OdGQB; ‘a [a?*6qZB. 8Dl11$\mathrm{X}$ ?TeFeG $>)^{\wedge} \mathrm{C},{ }^{\prime} \mathrm{Ygc} 6 / \mathrm{fXVXfjLO} \% \mathrm{~h} \& \mathrm{BS} 8$

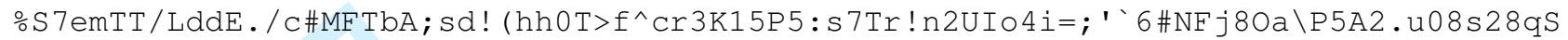
\#UD) bN\#IEK`*7K. He\#@SS

Xㅕ\&P" 4W] C9j=uj' 5I> [NBK: I 5Hk<2<Ts\&krCD\$C.CYU*<'P\%M*! . G-

$\backslash$ fh! 9c, U?O8u7Zns?ZGXGJ"F5Wo'\$d1-FA.! b=EC-<r'

$\circ \mathrm{H} .^{\wedge}-$

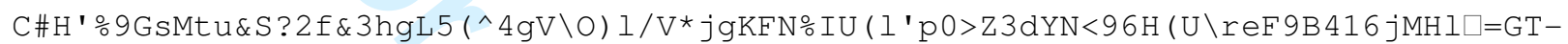

$>\mathrm{AF}$ i $5 \mathrm{~K} \backslash \mathrm{A} 7 \% \mathrm{H}^{`} \mathrm{UA}$

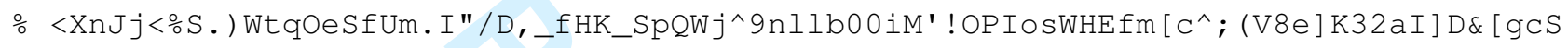
$\mathrm{H}=\mathrm{F} ![\div 3)$ elWf $3 \mathrm{~V}$

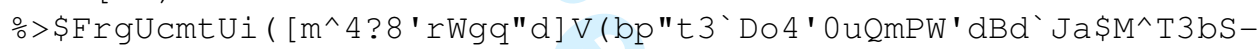

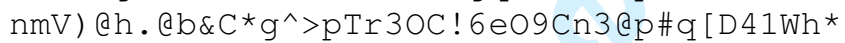

。Vu\&BLVD ( IBJCT^G_SL9V0D) /2RL@UR^L3Z, >V5KdKF\$O\&qIMbU3_`>) n-

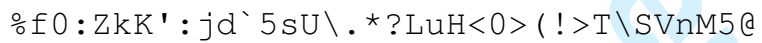

으, E6I=8Q1\#F2If] 9AAZ! \f2IDk ] CHIfHO>38Q@ ( "OP9BsB5c1M\&[ $\backslash ! 34 \mathrm{FQ}>6 \mathrm{G} ; \mathrm{C} \backslash \mathrm{FUJp}$ *iKr7JaX\#" aYtB2*BK5E\$`Y\&4\&/0f

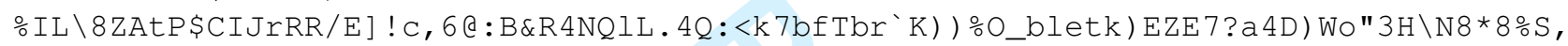
$0] / 5 \mathrm{Uh} 3 \mathrm{CA}[\cdot[\mathrm{VQ}=\mathrm{e}$ ? C

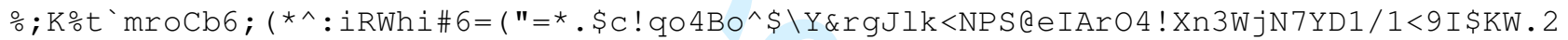
i $[(\mathrm{X}: ; 1 \mathrm{Fp}<\mathrm{J} " 4 . \mathrm{B} 6$ !

$\circ \mathrm{P}) ;$. ?GO0\&d8! $4\left[\mathrm{~L} " \circ \mathrm{S}(\mathrm{Q}-\mathrm{P} \&:<\mathrm{t}) \mathrm{Q3}: \_=\mathrm{m}^{\wedge} \mathrm{d} 99 \mathrm{CLj}, \mathrm{cSk} /-\right.$

\&j2.u:IX3\$icnk=nPDTCrY"cIZ4tcQb], [ =H\#06P2/>1XttQ) O] O

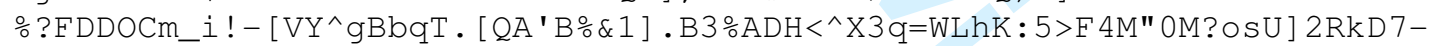

Y3bqjM, r\$OB\&Umj4UM; bkI`fKFpihV`*?

。 J*SOV: UXj"Y2rV<RQ: ) bM; D, kCm? [-

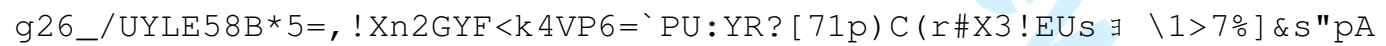

$\div \mathrm{D} 1[\mathrm{C} 83 \mathrm{E} \cdot \$ \mathrm{r}-$

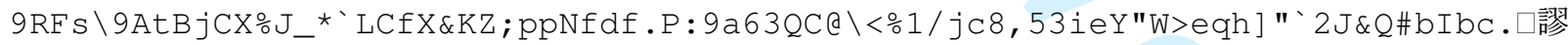

$\square \$$ ! N]

○8m70GW861, Cc80 (aU`QTD6!dk"tA [, : n\$Q7RP^CiRH0Z\#] <^_SBNkEu`V4AC5=1 [Kok 'mo'A [\$\#*BHi $5 \mathrm{~g} 76 \mathrm{reF} . ` ? \mathrm{Oke}$ ब $2 \mathrm{YW}$ :

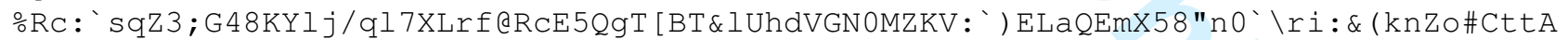
$\mathrm{BSCHm}[\mathrm{SDQGCb}[\mathrm{XV}$ ? Ok\#; $\mathrm{V}$

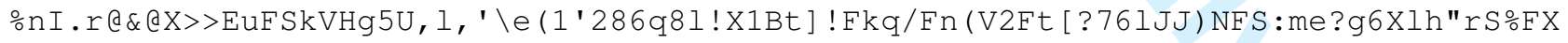
Eho; 4 SOfg<)X6 7oUSq:

…JOT.d $<\mathrm{u}) \_-\mathrm{K} 83-\& \$ \mathrm{ZjO}$ ?Km@`【緶

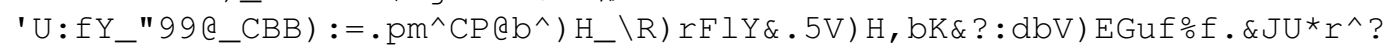

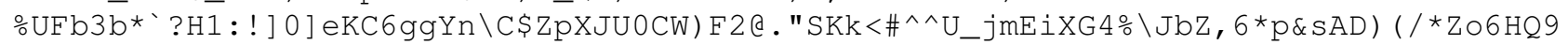
$\mathrm{Xe}-6 ; \mathrm{f} / \mathrm{fHZ}>8 \mathrm{q}) \circ \mathrm{\circ} \mathrm{MBCn}$

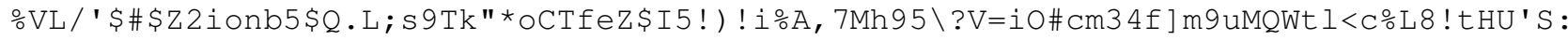
s5 [dLrVOXe3cpSLsWo

9 9367/. a : Gt) \#Z\$F; GudA>mRmW! 5uEB>S8 \$<9 ' obP [Ep.r\$”nePga95X! \#munC] KZ\# ( \$\&Ggl 7upCY> ' J $\mathrm{Q}(\mathrm{RC} \# \mathrm{e}=\mathrm{B} 1 ; . \mathrm{hZ3} ; 3$

애D $\%] 6$ eGYO; $4 \wedge$ F $<$ peu-

Y: $\{\mathrm{f}<] \backslash$ YRqakBe\&>DePrX<GeB!,^]re`ol OLr5V^6ZZ"N6 [ 4G 7r5\%QEJN" iNGK7?H?R\$H"Ef\$S8X1\%@E / 


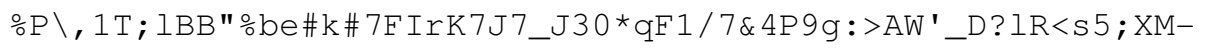

$: \mathrm{K}[: / \mathrm{O}) \mathrm{m} ? 6 \backslash \mathrm{V} 0<>\left[\mathrm{hq \# I} 2 \mathrm{u} \$ \mathrm{C}\left(\mathrm{pu}\right.\right.$ ' qCFOLTs^Dfs ${ }^{`} \mathrm{Wm}$

$\circ \mathrm{P} 1 \% \mathrm{f} 7 \mathrm{~b} 05 \mathrm{~s} . \mathrm{P} \div \mathrm{da}-$

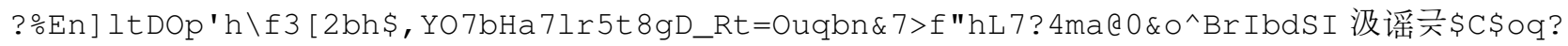
ㅇc\&Wb] " "` (2`e\WaXFP34n\$17\#\#TJi;d1\#i] 3\#"R"\$E991??, ; 1G, ] 8-

\$P6"HLff [CRU^] h] N/^,\#SL'_tkio, ! d^[fk, r.R66RP

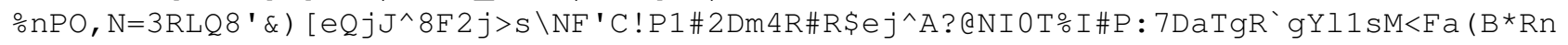
$\mathrm{BF}: \mathrm{Znh}]{ }^{\prime} \mathrm{OP} \mathrm{P}^{\prime} 2 \mathrm{RgUp}{ }^{*} \mathrm{C}$

Dm! iI9 歛 $T \square$ C $5-$

3K9oK^<iM"6URchh50G*7gatqj1D3Jfe65aN7U2! ; [ (3mauQNHmUY"^Q/20KoEDEHX; Rr" : gQpXI

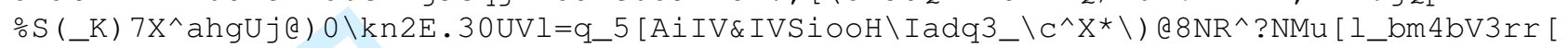
*3PQ1>BP6JQ[_DCUI \CQ

\% ( qbB 5b<? BJmg\$ ] qGK 4DFtnS1X LD 5\&: 7XX>UaY \DMBI. NGos 3Pn ( / $(\# /<! \& a=$.

-gdW] pp\#skIdH. Z1\$=rOPuA8tfE! ' . \[ \s\#hgJ4UEP (ngd]P.4gs`M) m\$걹 : u3gD4c-

m\#nAtBI. ) $>$. Ir 2 I ? eUs $<\div$ Rg $<34$ t $3<W$

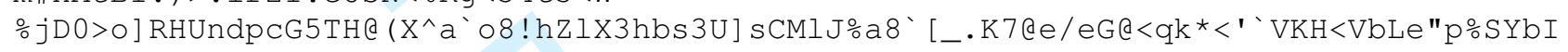
$1 \mathrm{H} ;$; hPhC $<2$, sgigjntU3

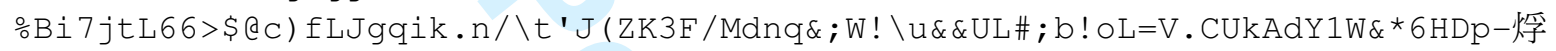

[jCY, ) HsVNPN9:; FDK.TF] E

○\# Lh^46HP\&p?<H\&b-"Nbns 图

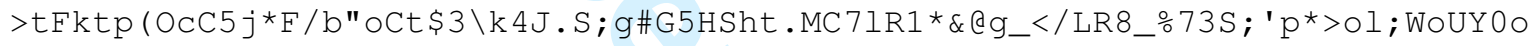

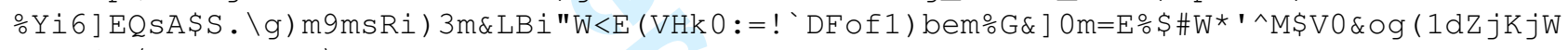
SN5J1t/N ( 'mnF??O\7m*-

。pRER. 2d?HO"8qaH^orED \b3Fqr*!p1-[_! 1<] 4X@2As_\$2J@0`aoBfb53QrI@qGTnEXo8/I '\&j0gIOQNKqr5H0\#5U@OW, SUP" \&W

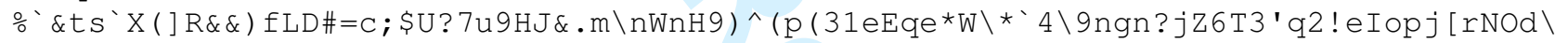
$0,4 \backslash \mathrm{dlA} \$ \mathrm{bZd} \$ \mathrm{Kk}>$ WOul<;

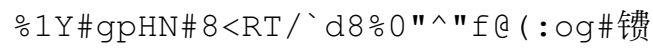

$\square \circ 6 V p \#$ ?ute05Zd@; 8aEY) O=\HUq\&h_krK) C_KsJPYB (^bq\#CuNFaNOZ1M7t83 "R] Vm

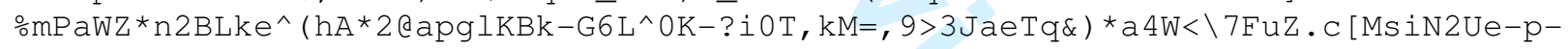

$\$ * 6 \mathrm{XDZ} .1 \mathrm{Bs}[" * \# \mathrm{~N}<\mathrm{q} / \mathrm{kM}) \mathrm{M}$

ㅇ ] F $3 W * P 7 T F A 77 e j J X f 6 I: g W 8 X 9=V f B ! g=6-$

bQ) n3Q0D1H@Id [ I> ] h9 [ 7*jXf9M94u, @.3 (W@@?Do@R: ' i (iBPi9NfEL?*\$?D/a">

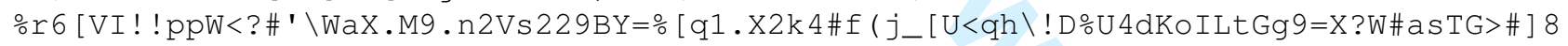
QW) 5 Dr 1 " $<$ Z\#LSPO $18 \mathrm{f}$

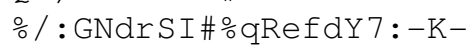

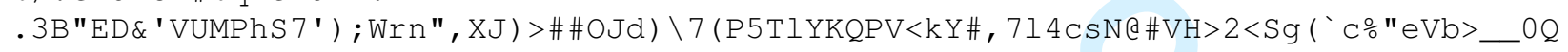

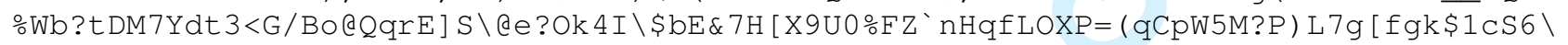
! ?KIS $8 \&$ I oue $0-\# 7 \mathrm{mZA}$

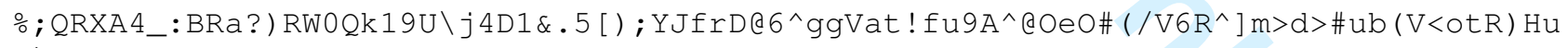
(d) $\mathrm{eY}>\square=\mathrm{XH} 3 \mathrm{ST} 8($

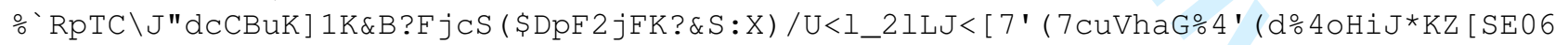
$\mathrm{n}[\mathrm{G} @ \mathrm{RP} ? \mathrm{I} 9 \mathrm{kMMqtK} \mathrm{O}=\mathrm{S}$

을 f a / \# * $\mathrm{BU} \div \mathrm{U}-\mathrm{T} . \mathrm{d} 2 \%$ 'E5YG $\mathrm{mhW}$

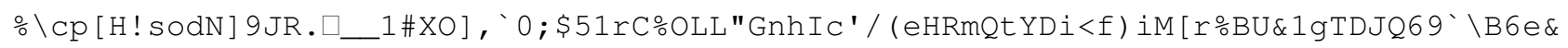
$; "=-\mathrm{CR}[\mathrm{YOg}(\mathrm{fH} !+3[$

? "C8r\#k@S $\backslash R / G s V D c f-[e 7 G a L Z Y f n A \# ; e 0$ 庸

,H/^fC.'2\, Z?djHMa 8.9M) EosM[U; SOO=_ao\%0eC4/B=(\#R] D@Rg_.OiALL\&@

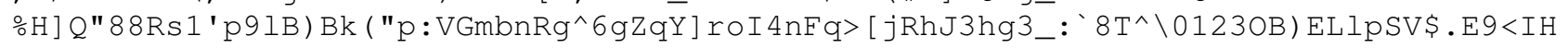
$\mathrm{kmWM} \backslash \operatorname{miuIY}(\mathrm{bmS} \&$

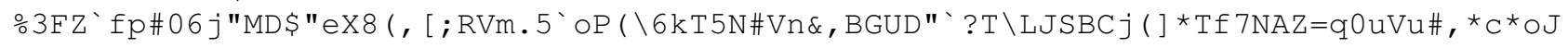
$\mathrm{k} \backslash \mathrm{F} @ \$ 1 \mathrm{~F} \% \mathrm{R}^{\prime} \mathrm{RV} \cdot \mathrm{QB} \backslash ">\mathrm{hWO}$

URL: http://mc.manuscriptcentral.com/tandf/tmph 


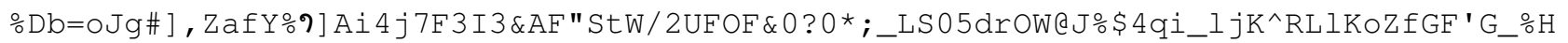
$\left.\mathrm{eH}^{`}\right] \mathrm{hTg} \mathrm{J}=\$ \mathrm{DWrbP}$

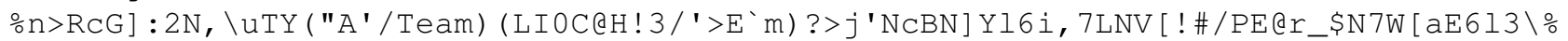
$\left.\left({ }^{*} \mathrm{~d} \% / \mathrm{K}>; \mathrm{e}\right]=\mathrm{aMB} \$ \cdot 7 \mathrm{FN}\right]$

\%WYa@TC:t=SZE/1@_qodbo16=[SN4I=,; osrofr 70 ?kmUHniJGP-

:6E/'\&`O7J0d!" (?mP_Hi3MbftR@FNO\&I^:U; u) 10_n>mM

X.XaIIe2L $\%$ Qb) gt 9UXXDV_n2"jQG>mAt', I. I0\&55aT : : P ! =-

(2^H5qg.K'OU] EQPp1THR\#9D [^_f, .N8X?JH. qpLZUl\#iGUot5?

이이 賝

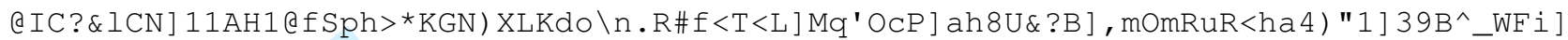
$\mathrm{OHmO}: 5 \mathrm{GZTBj} \#$

으

ns! 8<hd.B/d "jSbo!raHKhoK,Y; ?g6F" ' ! o1X[! Z] [t9 ' ) 79=V>;/^@CO851Z\&U] ; Iq0OE: \$QT_ZQ83 SsrA86_3! NH5Pq6Q-0

․ RJL! j) ’PWb-1ShaQdi-2lJf^B\$6r7iG3\$k\#o3h3M2R@e7j@nHhe2, ] Rd7Gffh. =`d[Q2e'Q( (fOsIepi<a`e\#VZ.; I $4 \% \mathrm{gX}$

○64ro5LDfS [Vs\$'!3@k. ‘Lnh.1R31KVDSbS\$W_ONhpLuQ) aCVn4GdX] \&Y] N (ZJ02kiB), YIIbb_>uP $<0$ nV2aJmlRp_oe $>W<Q g r N j$

。Sh9"CFDG:5Wg0e] $\exists$ 栽

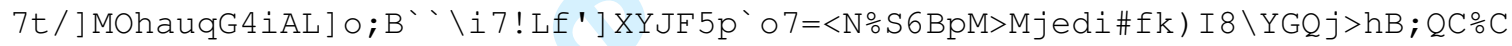

\% \&me' 90 ? nmn*\&em-

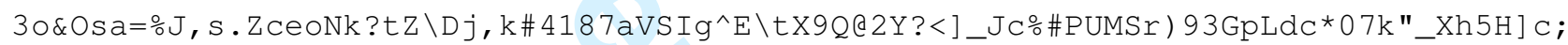
$-4$

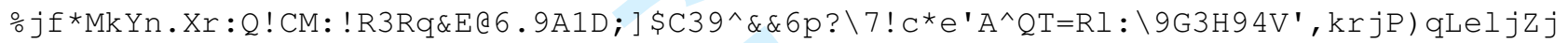
QC` $8 \mathrm{~m} / \mathrm{b}$ `- $55 \mathrm{Hba6qFg} 8 \mathrm{~B}$

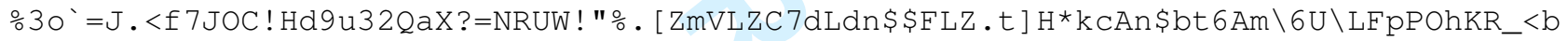
u $\left.9 \& \operatorname{PkN} 2 \mathrm{bDln} \mathrm{n}^{\wedge} \circ \mathrm{mSHA}\right]$

$\div-$

Er=bn" 8V/9*VRq8XVa1FOMumDd\$JPi) i\$0P:31\$E, .PIp5V:c^S9US) 9pZn1'j\$2/\&)!coeWMEbcc2^i G\&Em'iAT $=\mathrm{C}] 7 \mathrm{qC}^{\prime} \mathrm{T}$

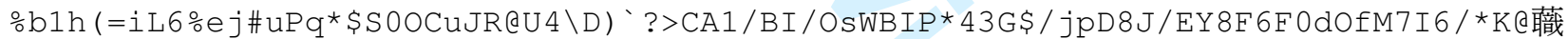

'e\#M=N: $1 \mathrm{~L}=>[\mathrm{hW} 2 \mathrm{~N} / *$

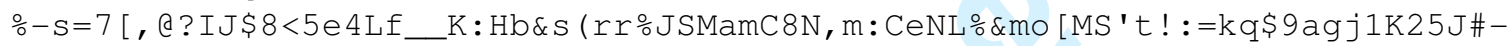

/^T2rshdmG/NhokI>LXf; $\backslash<\mathrm{aIo}:$ QS

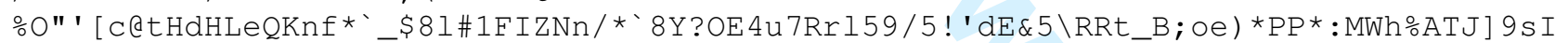
$; 6<\mathrm{dfuEH} \_\#$ [ $\left.^{\prime}{ }^{\prime} \mathrm{Rg} 7 \& \_g\right]$

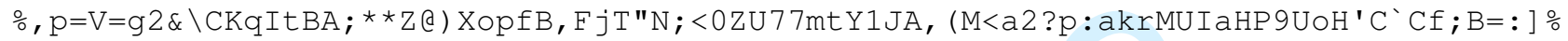
\#O\&@g^Z7pC9^ti, ) 2RL

ㅁob"39S (o.rf (55d!_1s ( Ie\$3G OYEMlSKq@V, 7! Eg5Pf5qWN-

$7 \mathrm{rhMKk} \& \mathrm{~N}=\mathrm{VIaX} ;<\mathrm{dd} ; \mathrm{hn} \% \mathrm{P}^{\wedge} " \mathrm{X} \cdot 1 \mathrm{CC} \wedge \mathrm{HG}<\mathrm{Li}=\mathrm{Y} 4 \mathrm{pj}$ OJ-qVZPu $\mathbf{\text { a }}$

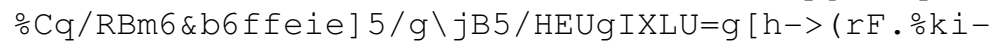

ob\%@hfBrZY" [1) jlTLpX/mTpM3FZR; 1A\#)] nLmL?TI (90[8ajifW/

가 ] -Sj1\$Dl/VX\$fN`^m1VfNX_D] ( [ ! Amo (g0PonU8jhunM=aRgCQ9\&Ba"J6pMu70) Yr/S-

$\mathrm{CZ7}=]^{\wedge} h l k \circ \mathrm{E}\left[>I u^{\prime} \mathrm{M}^{\prime} \mathrm{q} \backslash 2 \mathrm{npYA}\right.$. $] \mathrm{Ipl}$

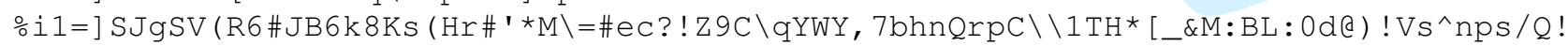
:LYS $4 . \mathrm{Xdl}] \mathrm{cKj} 20 \mathrm{~L} / \mathrm{kE}$

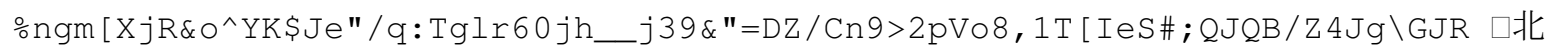

$\square \backslash$, bhAOn! Io-?`fFu

$\circ \mathrm{F}$ (iuV?r-

G [ !r\$\&3i5G\# [ 7PO>bJt?\&P@u8N*/B@ '\$gJ, * \Ehh \>b\#\%hYm? Ir \[ IRgKf8ph\$, ] VCC>sbSX'8] UeAWm qG9sY4if7 $\backslash a^{\prime}$

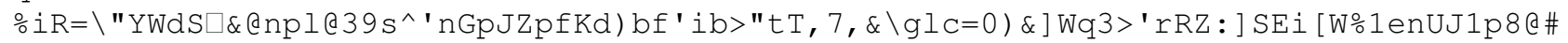
$02 \mathrm{aFdV} 4 \mathrm{NG}^{\wedge} \mathrm{L}<\mathrm{S} \_\mathrm{QP} 16$

알 : / ) G. 1Aj21FhM/J5>MY] 6BeuP' (p'lWhZXhqP-

$\mathrm{T}^{\wedge}=$ WSqg / ] njo90buSZ>? ( ld>; bfW! Ld8hJMrZ: g) bp; / 7emMo"C\&4eIW.1 


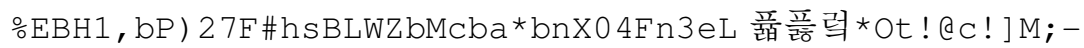
h0\%\#g'] lu> ! 9k [07>2Ef4] [K9] 5u9reAg-3>;G\%'S

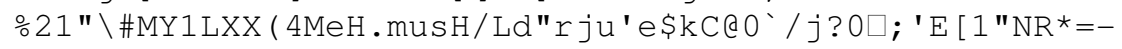

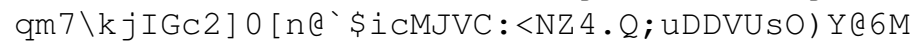

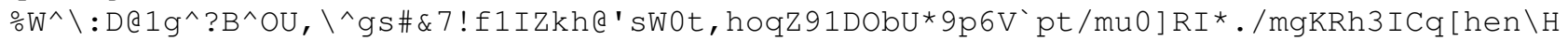
FhbuD, Q9mQjRpKDt?P/5K

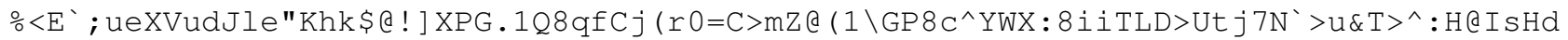
$>$ BIdi4\$\$MIUIe: )^ $\mathrm{b} * X V$ ?

○! MqH (\# "ORF, gKY>UT \n\#_T?b1HP2n $\left(\mathrm{MW}^{\wedge}, \mathrm{MO}-\right.$

PKL`d.p: [UiA2Vk3Edn=!F\%2=sb?\$4H6, okmBfQInBEq\$Lh\#B\or6J, (BtYPIpG

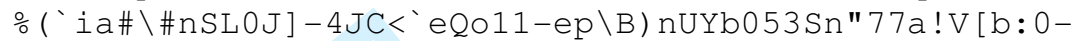

; pXS\$k9Wt ( 8R6A (_\$) bZ, bd7*EDJ ( *EIMR! O/ULrqmb5_XeiV\&

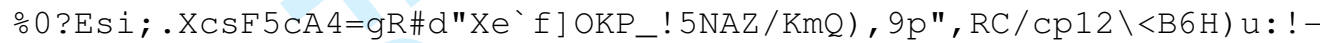

'K5i`Cflb>S: ij3: inM; b5<g"Q<m@h@a4I] =

。 (mQ38"EFY8D)^\&uetEi-

3U! C) ig)=dPZ! @e25L84kRmnL:kr7BfG3V\#A9PgKa; j90\&GQV=O@Q:^:A3 ?Mmh@M: / juf . TFdX\#7U\$ [ \%j\%_gWeeKWZR`3, Q_HBYq., ( 7 IRW=t5 "LZ_d \OIOk?16m_5dXk3JXRttp\%`Gu*\#HRXnkG: LUWd!S $><\&<$ $\mathrm{n}[\mathrm{G} .,(\mathrm{VmGOkY} ?>[\mathrm{ApV}>7$

OH"U3XNTe\&'RsA975PHG\$.S*":K\SZm\#e-

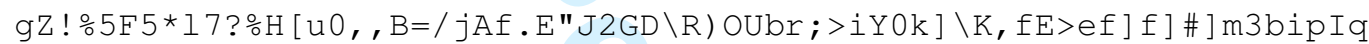

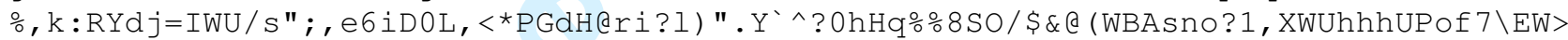
G>hj\?] ] T49KpTlXLUpaQ

\%c ] 8enDYHY, H] Vp (k5FLmJs 4 "e>\&uRYB=\%"hEL] / jjImXj :_! 21G\#"Dsoc; D\%h=EN-

; \Fg9\%4Ml*^YPhELFpm, [^4FiH9S $\square \#$

$\circ \$ " \mathrm{t}=) 0 \% \operatorname{RPVHYf}[\mathrm{d} 2 \mathrm{~m}(\mathrm{bt} / 3-$

$\mathrm{U}<\mathrm{s}$ ) ! / ldoY\&j : . FrV<Ul4\$ ] qlqeqdWGUHt2eQAbl 7Vi 5e3^P@BnN*/1\$m (r"uWPn2\$gs] 5h9Uo3U

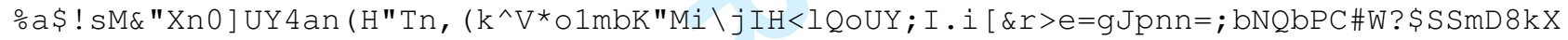
"YO; $a \mathrm{pB} \$ \wedge$ ' $: \$>r d D: X \backslash I$

$\because \mathrm{CYKO}=\mathrm{ImJOEUF} \perp \mathrm{D}-$

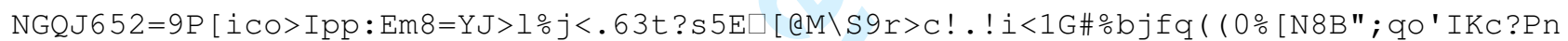

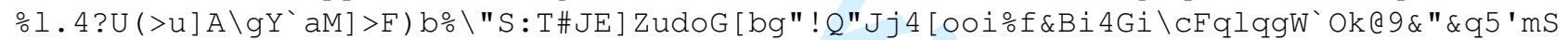
g81>h!s $(\mathrm{mRgT} \div \mathrm{SBZ} ? "<) \mathrm{Y}$

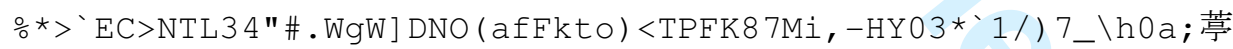

파> ? brPaK/? TgX: g\# $n$;" $>, \mathrm{VT}<\mathrm{B} * \mathrm{k}$ Se [O) iPA

JV $>C:$ : $\mathrm{Eg}=4 \mathrm{~B}$ ' $56: \mathrm{ms}: \mathrm{g}^{\prime *}$. YGIQ., En.b-

EUhnMSQAGKKHG 14Q0*, (m7) U \2:HI !rPp 7=p) STC\&P*A" (B/8TlL 4f2d\%:895"0q

5-j3dhoQF65, VikrJCtp_O*C7VO-XW\$tsh6-

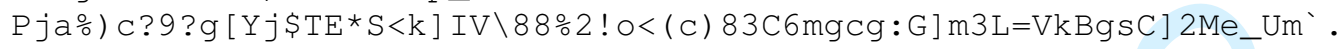

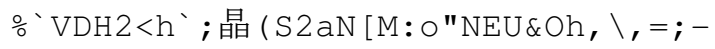

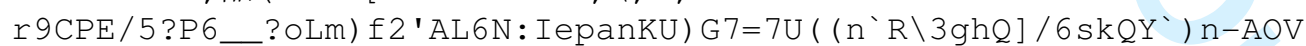

JGT2fMfEBI4HgiP2L-F) @ 73GinVL.bj ( La?Tb=h*Z\%F9 je\$akb?QJeorV.b(5FA) c5m-

QS [H] g0tHq\%\&I@lgJ.s?YO_[iHk, ) n

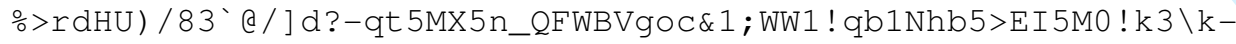

$\square=f e k Y ' s^{\prime} i 7 u A 3 Y V 4 i d: S m$ ?pXc@! joaRofanE: 8

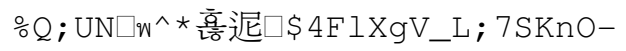

$4 i<\& 941$ IUmEY) >3Y) aqGM] / ogL, oVfpe 'DC\%I9Y*>fmsfr_1 \dH\$\&mW6/N@j

K\# ?GTY3 04 IJK` 3 i 36 : \$C (g?ae) ?uqEHu ' iBB8p\&KKO, nN\#enSQp : 9-

6. $7 \mathrm{NmFgppeVbD}) \backslash \mathrm{G}\left(\mathrm{t}^{\star} \mathrm{rbdk} 1 \mathrm{nn} \backslash \mathrm{bW} 7 \mathrm{PL} \& \mathrm{~V}\right]=j \mathrm{FJC}[($

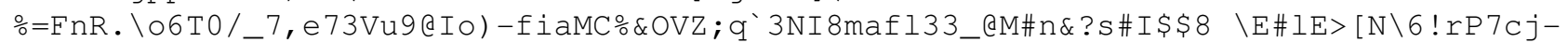
88 rn'0-WE; XtHraY! \%

lmFH/b_eMt529KH6 (VKmXs*n [ ; D_Di. [ JXueCqQHbd, \$MdB=6] / S<KO\#\#F\#=YZdVj; *CC*

AKY8RHt?: 0 (GYJZC' oPNaEkLHFg

$\circ \mathrm{qD} / \mathrm{p}) 3-$

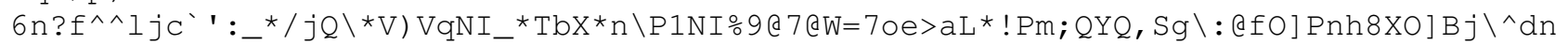
$\left.\left.\mathrm{FPT}-/ \mathrm{S}^{\wedge} \mathrm{kPi}\right)\right] \mathrm{b}$

URL: http://mc.manuscriptcentral.com/tandf/tmph 


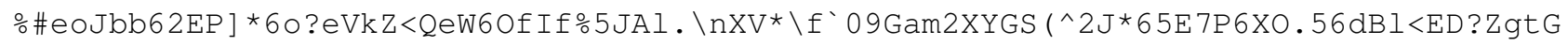
S6G8\%VfPLB2I’ $\backslash$ fqFgn

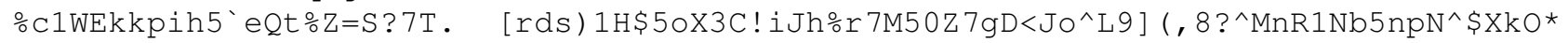

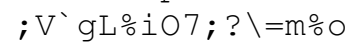

ㅇJ35Z, [V-2P*C ( (-=brkadGm5GcEm?Rg) hATUZc;\#Sp6 £。qm\#pEKb9fHGuF?C; , cmYsd^, 0-iW?ft"$\#: 9 \mathrm{z}$ " [O^tg $4 Q @ D l u-\wedge b .<$

X.XrI-5-6-

J47\$`E.o@fZGA^C3\#Z\%"*L@b>/.1Sk"! 0>K9pa"X.1/, sQMIB`"IWieO=PFLV9('5@\&?!CDbj5_V>) ' $<U U S W I I V ; J E$

! !CQ:T\&S< $\left[R, 1 \mathrm{~W} !-\mathrm{e}^{\wedge} \mathrm{PX} 3 \% \mathrm{OMD} @ \mathrm{KFr} ! \mathrm{QjAhH} 47 ; \mathrm{eE} \star 1 \# 6 \mathrm{TA}.\right] \mathrm{TC} * t 4: 0 \mathrm{dm}-$

1 e [D\%Ts $89 \& S \& d F b>$ ZDALIfgj9 [_-'dpdC, H15^Eh\&R3

○KL_Fl2Kq ( ch1\&OjJKCeb?acRmgrMdNeH5X-\#] sY*UbSNe92>07'fjgLcga/qDi? [d=0 狤

㫙 $\square \square]<8 \mathrm{C} \& . i 9 \mathrm{~V} ; \mathrm{M \# P} 2 \mathrm{TZCZ}^{\wedge} \mathrm{J}$

2? $\left.\mathrm{F} \backslash \mathrm{Wj} \mathrm{J}^{`} \mathrm{OH} \mathrm{H}^{\star \wedge}-\mathrm{YE} \mathrm{E}^{\star} / \mathrm{bT} 7 \mathrm{i}\right) \mathrm{D} 3 \mathrm{CI}$ I-EboIFZ5J5\%AKqQSSS-

^r9FULOp`ea, 8>VD; $\mathrm{p}=\%$ Yap\$4DnIGEZM; mE\&q`OrT85X9eQWWVAQZ ) - ,

! ! ?B6YLO2OTU8*ZO7hqlEu\#Fskuk [U`ffBO\&P\#P1n4e\$\$1'dgf?u37k" qW:0P3EgR.R\&@N; W\#R'< [Uf ; UdPMi01^B 7) XL, WH- [Wg

유/hILqLH [V3<sa`_NQ3f<GgZ!R9 \=. (nFY2\&=CboVh) ' 9 ?^nCGQ; K [ ' -

\$ojUjo_') gWWDplh_Y[en_mH (2JibroOLN' leQ\&\%H/kl

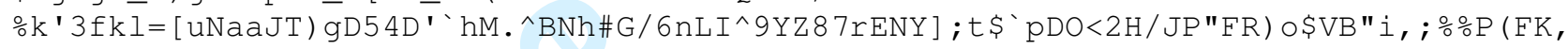
Kh0> ] sYla;B\OVZ, b9kZG

을 e\#AbVcNDUl fPf [YobEMP\&ljfd ( 4 j\&IhWojAd3Aq\$OIi\# / 04$]$ WKHn-

Mr2RHk"3KeLuu9D_MI_; ) [Q=[BfiPZ?S` $\mathrm{NW}^{\star}$ IUe<WZ:ge

은 3 ! i^1mO\&@.PRF $(0-$

P] R\$N5 (<>7*9oCm9iMSUNi! Xk\&n<BU7ZeZtNch^. BPZ>Ok>!^X_'n2cG>0^T\#OEGYnPIu> n5P\$-

*) $r Q S=$

○8`Lnligo;m"j:u/ $\backslash r J p 4 X) k "$ 急

\$\#; ] u>7. [.Xm_AH (=oeKC=K\#HLPUIIIUU7W@H<BM^Zm:W7PKhgpBe\# IF9Xu5l@ah\&2r

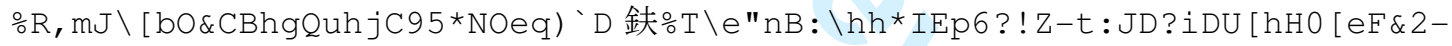

_RPH<3J8j.C5`fr9/PPY4) FdP9ZBCk

Phnq4QX.G\#k\&^; hsG) "IMU@3IaF' ar-

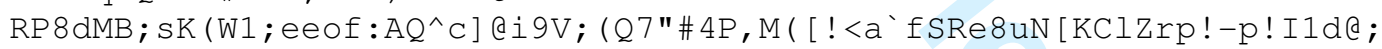

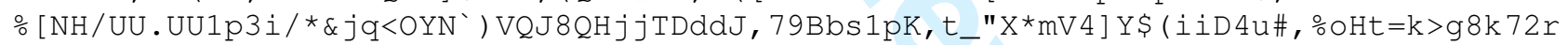
$\mathrm{U} \circ \mathrm{ncM} ! 2>a K 3 \mathrm{pY} \& \& 2 @ 4 \mathrm{u}$

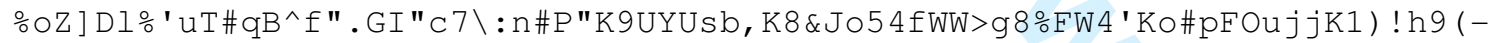
: @130MDY_NYCOVQ_QV^!C; I2Woa (.0

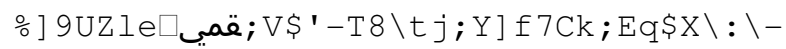

8F<Ej<"Ckg83'k?0bDr^L"BRt>\&?!khT@WVVVti (\#LpXf?02XdH/A/응 T [K2

은 $\left.\backslash T^{\prime} ?\right)>-$

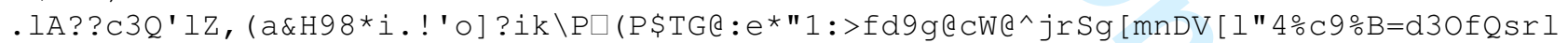
$\mathrm{FK}=\mathrm{kh}[>5$

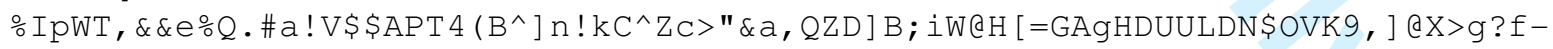

SPEPjr.;rPARs; IbqnT_6c>j

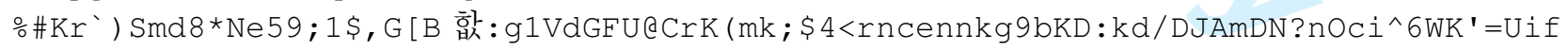
up"YP2H6jMfo"a: !V $\backslash$

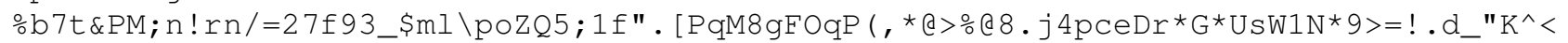
$j 1 ! \mathrm{aH} 0 \circ 7 \mathrm{fR}>8 \mathrm{U} " \mathrm{t} 6$ ! S

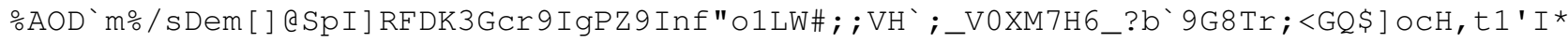
$\mathrm{Js}{ }^{\prime}>=\mathrm{Ake}>\mathrm{jA} 8 \mathrm{C} / \mathrm{JJ} 2$

○gA'ri( 'ZAC6Hu7\%R82_E>5lMUZ3?^^-=) 0K06-

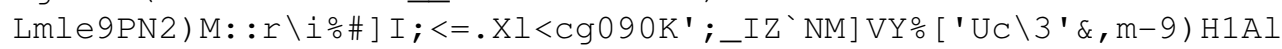

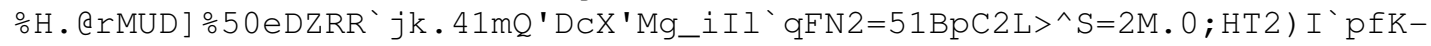

ZiF7IfBCE" 8U'e@\$rngm0eMa0E8e\%^: [

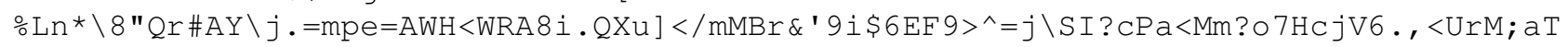
$\mathrm{S} \circ]$ ] $8 \mathrm{H}^{\star} \mathrm{QTSWF}$ ? fXMh 9 fr $4 \mathrm{~T}$ 
D 7 ' CaKpE [_POCe?\$-

MWB/s [ jtiR09 (SN5G*\& (F0r*, Db [ ) 9JFHT [ go4gJeN=\$3H6>9mP2O<[cQ\#\&Yu; ci0sPJ"E, >S`stdcIF $\& " \mathrm{~A}$

○b $\backslash h / \wedge h\left[\& r ? \$ s(B \backslash g, d ! r j a<[b] C(m ` r \%: ! k d=O F 8 T a @ 35 X] I U q . \&] J^{\wedge} . r * j H c j ? A c K(M \# e p B @ X X E 4 H @\right.$ $\mathrm{W}$ !) $\mathrm{Nd}=\frac{\circ}{0} \cdot \mathrm{U} ! \mathrm{Gd}=\mathrm{dg}$ 鮕

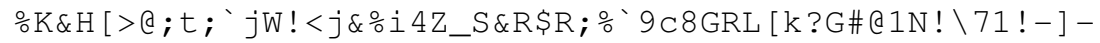

$>$ ! \#I>GHM_V?RZV' $9 \% /$ eeBs58Uo:C3-h_4Jq(^^kd.aZ>3P

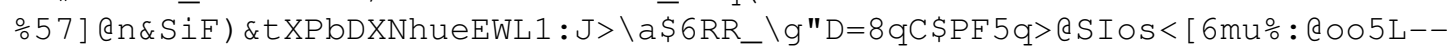

(\%Wi]dZ'DDl2] 9r] Me8WWFpg`GV] r>OT

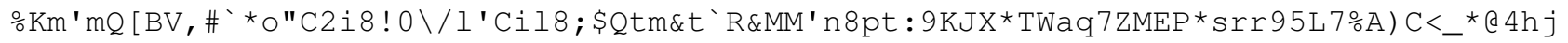
$\mathrm{Z} @ \mathrm{WHKCOD} 1^{\wedge} \mathrm{F} *[3 \mathrm{Vq}(7 \mathrm{CAs}$

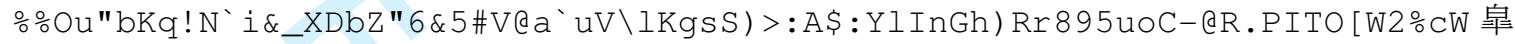

$\backslash e I e K W m I K N \cdot j D j \circ X 2 V p R \# K($ ) q $>$

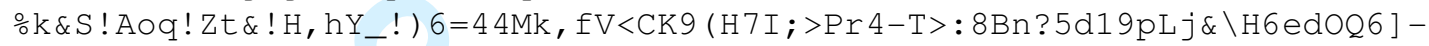

rAOHCa?@iK"\#'Prn_).^4mะLut>j6\&Z

X:HWrdqW1a, i49no_[q*39[ZriII:F\#Q=<j<YPkc30P07_/SKk\$口 ? ?ml3\$0tI_ $\square$ 代

\&p0o7XVrk \2a $0 \backslash \mathrm{M}(\mathrm{Y} \&[$ :

ǋ ! f 4=66=3XR506>XROJBKo 'WN\$HV/1AZNSk*\&7@; f<16i!rkV`。45K6kG8aOU^BBPj! 3 ; ] -

$\mathrm{L}=\mathrm{W} @ \mathrm{~b} k \mathrm{k}, \mathrm{tK}-@ \mathrm{RebP} / \mathrm{m}<\& \mathrm{Z} "$

$\div-$

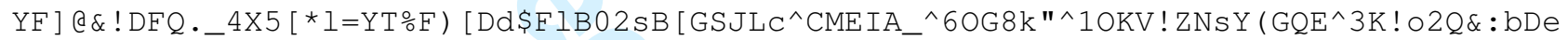
$\operatorname{XrBd} 1^{\wedge} ! / ' \& W \_n F 1\left(s^{\wedge} i\right.$

니 $\backslash-$

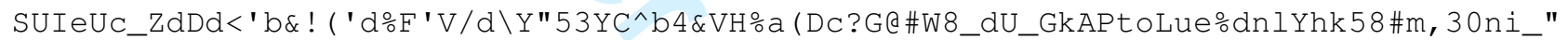
ah`4ql'9T:KYP\$d

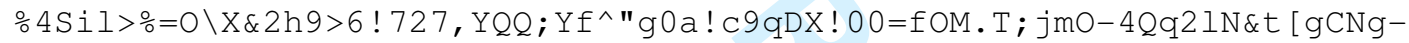

\#Rk\#6\$!ch) c4) 5 ar, PrQ<\&<`iE [^b*W

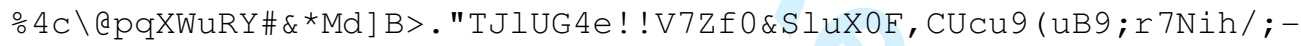

$\mathrm{L}<1 \mathrm{Bqg}>\mathrm{p} 4 \mathrm{QqQ} \mathrm{Q} * \backslash \#) \mathrm{E} 9 \mathrm{~s} " \mathrm{C} ! / \mathrm{D} * 4(\backslash \mathrm{C}$. I 筎 [`

은 3 leWnJK $>$ ] Xp^9OY-

/UOOWP8gXZTaH] OgCtPYh*PQX0N\$*'T!Yq`3 3@NHo\#jT5c: [dd8EnZL? AN [935IP-

! $6 \mathrm{JO}$ '\$Y@lh\#DD $\backslash * i$

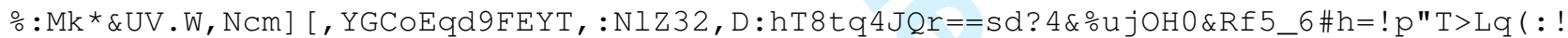
TEM: $₫ \mathrm{XX} * \star 2 a>^{\wedge} U 5 b^{\wedge} i M$

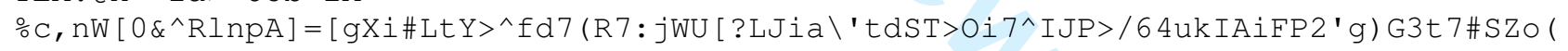

$\wedge \mathrm{S} . \mathrm{D} *$ ! SZlR93goB\&\&uGma

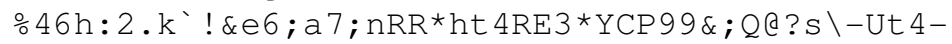

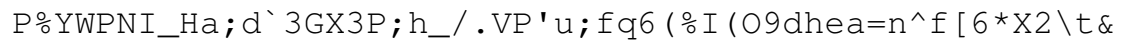

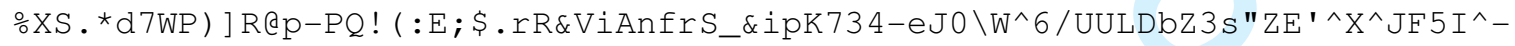

$\mathrm{KN}^{`} \mathrm{~V}^{\wedge} \mathrm{h} \backslash \mathrm{Om} / \mathrm{f} ? 2 \mathrm{R} ! \mathrm{pqR} r \mathrm{Mk} \mathrm{ObQjuf}$

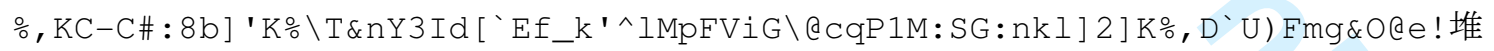

_iexfin'jPpH5MDP:P [CP8

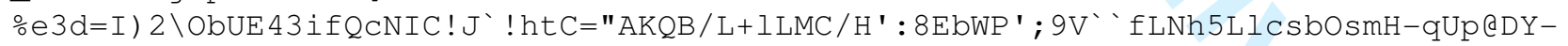
$, \mathrm{OM} ;<\mathrm{D \& B} 4) \mathrm{Cq} 4[\mathrm{KeOU}$

。Ea*I_mT"gP $\backslash R 7 j 7 \mathrm{TFCD} 0) 3 \mathrm{Yn} 4 \mathrm{FO} \% \mathrm{kRk} ;$ ! 4U\$.M-dp2) \#9nM*5X2bNF 2Os -

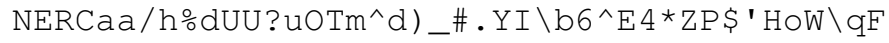

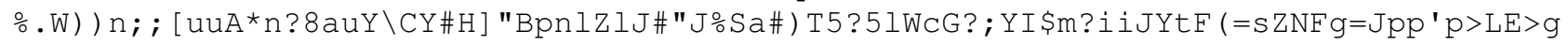
$-57516 j<\mathrm{SM} 4 \wedge 9 \$ 91 \mathrm{~N} 3 \& \mathrm{U}>$

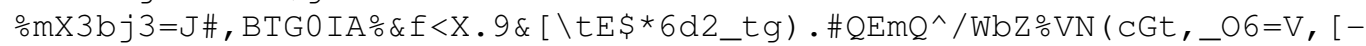

53RtXBI; 'Yt $\$ T @ q \& n q L F] \mathrm{T} \& \mathrm{t} J "=\mathrm{CG} ;=\mathrm{h} 8$ ( fq

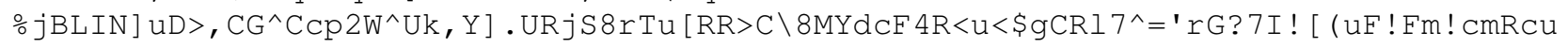

6 舅鲄, $\mathrm{PYW} ; 3 \mathrm{P}<\mathrm{j} 1 \mathrm{P} 1$

ஃAdQh] 6N [k9LQ9cO@, <p62\$O"HX@GH"UI<I<CSX:RIVQ\#i [ sGSV97:R`DG59b5g25103_gsher <G) <k/ i-d5KeT\%e==P-IOHR_Si

응 jBn\&agHWfEJ ! \# ' $\backslash \mathrm{H} 3 \mathrm{dN}^{\wedge}<\mathrm{A}$ \$C\# 8s\#N/N<pa53?G! JOR:^ 7W: Quq00 ] n] blaa\&bL/, o5RpTIRMo5Stk/ WTgZe3 $\backslash$ HC_AbMN / \&I_p-?

URL: http://mc.manuscriptcentral.com/tandf/tmph 
。; $A=S \$ b C M n a \&: S H n C N, " \# T * Y, \wedge 1 M K C 2 * 3 \# I \$ C S a$, feGrY $\backslash T 8 " Y 5>K \star \wedge W @ u 6>c: S m j 34 c\left(" R \_* I " T R s Z G\right.$

$6 \mathrm{D} \backslash 4 \mathrm{n}-\mathrm{JXCO}\left[8 \mathrm{C} !^{\wedge} \mathrm{A} . \mathrm{I}\right.$

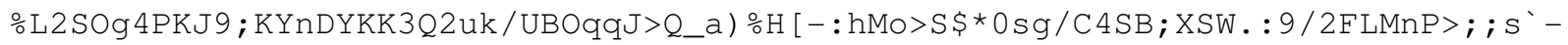
$\mathrm{ma} \bigcirc 0 \mathrm{k} \& \mathrm{qC} \wedge / /$ \# $08 \mathrm{k} 4 \mathrm{a} 7 \$ \mathrm{Ba}$

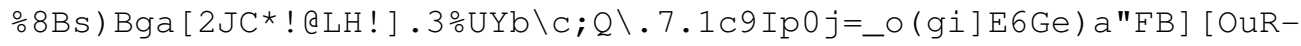

$\mathrm{N}[\mathrm{s} 8 \mathrm{Va}<;$ qR6 7E"33Cs"WBS\%UR2 . \$qn28DjVFL,

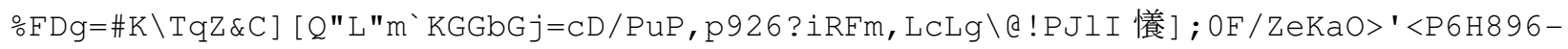

] ] N I I $=\mathrm{J} @ \mathrm{ChP} 9.00^{\wedge}[$ ! tX

⒊SVO ( ] [YGh5OdhIr I \$ ZMFra5ime=6 TA8 (9MJE>3ko] \#6d! 9Us`WF30>! Gn\&k93jsiI $\square: 2 \mathrm{~s} 65$ "^*SAS1

M) ] I / G?OPL] @dPH

TD@ $;$ Di $\backslash$ ) gKXg; ikr<"RBP1i 7f' l [1M: [ak (XF5J [N8hU' o\#a=UmjJ@PYCS $<7 \mathrm{~h}-$

"Ea3! F2kbghp9\&Zu`醛@4 $S^{*}$, \#l, 2J; h

으/, 3<4_>ikOtCp, QcHB 簛

$<=5^{\wedge}$ ] UUCk; ) q'MTLqH@On.m:7d\#f, hiLT.pZ1S`Jb) E; \#G7QPsE (FU] Y>KI. @UU3BMhBMbE, 7dR

。CrghYjoDRFC:mGGHLM6rQRT\&uY'! (RYtWm4\# \$9"=;597"' $\mathrm{G}^{*} \mathrm{SJJ}=<9 \mathrm{~F}>$, ?t ( Sz8rPrW=a@f) 7 䲴

_pu_! $\mathrm{Q}-\mathrm{qE}^{\wedge} ! \mathrm{b}(\mathrm{HOa} ! \mathrm{g}$

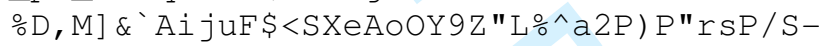

$2 \mathrm{KXJmWU}[[3 \mathrm{gK}, \mathrm{J} 6 \mathrm{eb} \backslash \$ ; \mathrm{F},<\backslash \mathrm{Yd},(\mathrm{BOk} a W m B E Q<\mathrm{Jhn} \$$ ) flNn9mLHVQuJXV4OqI

ㅇ j " 7mrM7 \$WWRBGPe jNq^P [i>\&] NB"HDW6d8ZYrK0 ZDo=@f 7SKC? 8ZqT<k90?Cqj 4\$ hF $>\mathrm{FM} \$ \& i f E V<8$ ' ] IKOBM. O^d78Z"GB9J42W

○S>C9p65j]VTCQ_Q\$9! Dqf0k950U_gTVJJKOnC_\&Oq0OEK4NK! k@KOB<?C ! ? '<PU! @Xa-

jnrGkbuW) *ANJ>VQsQfF $5 \& \mathrm{G}:$, : >PH@0)

\%'] $1026 \mathrm{CuN} \backslash 0 \mathrm{Q} /-$

J1AHu 'XZK ( 74g/" @4S?@ihk, PbnQU, JO>\#pf_O[=p2A] @gr' 'D (8KjuXM*' tpHuBdhM9W\#p; NE "XmYIG $\& 63 \mathrm{IaW}$

ㄷㅅ․ p.GVkIdW65_q0H4/PI4sn0H/HZ5-

o>*, S5S! : DNkJn>5" : fN1SL [1hBIud (f\ir^uZ/pKUoFEU52?PQ (mXoYCEqRk\&q9`J

$\div 9 \mathrm{~m}$, ot ! Hbj-

$\mathrm{Z}^{\wedge} \& \mathrm{O} a=\mathrm{Cl}$ J J=9MfFR?O\$GmT ' ( 8V9WaDV:F>68\%;C/L>6_<Yj/CIOPaaBSps3nJK4rUEALC2;RY^9_7, 10 *tAHB32Yk

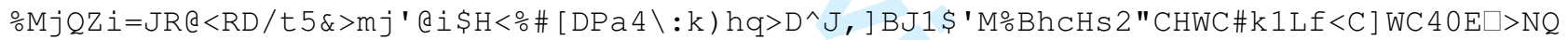
$\left.\mathrm{db}^{\wedge} \cdot 4 \mathrm{LP} 7 \mathrm{ZdU} * \mathrm{UST}\right) \mathrm{R}$

○'6BBFlanE' 2\% jdH>hI0 [ J6C4 \$qa>G! QH0! fBN'e04Z-

$\mathrm{ksH}^{*}><\mathrm{G} 8 \mathrm{ZI}$ !?_83]; $<\mathrm{I}$ ( (O^J7auPA $>$. \A6qo) (4GS" "TAnjk\&`3CeLJU

○Q,XYbJ . ! <U8 [S4W_Z] 3hdO! O/2_Kl13' \#J* \K9k\#@C?Ii *>#2i\#rZJ\$WPb1\#T0Y=N\$Ks\&., $0 \# M j Y 16$ GOD"jL2s: $\backslash 2 \$ 1 f 11 \mathrm{Pk}^{\star}$ :

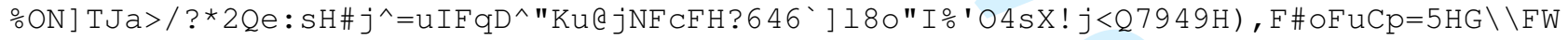
F2gKXTqkTB/Qce@6

Dgu"CbG5gs"b.bt\&N<Y [ [HfB4Q: CbnO! [2Ns' -

eWeDkGr.i@_lcemCIrNF : [h'FP=Dk> ' ?, l] 3GgT] ph7S\&OsR8; e\$qRW1W9_:_Po

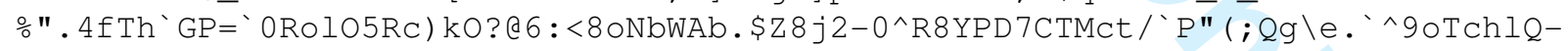

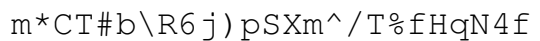

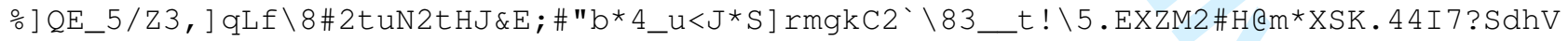
KC_E! - 7GP (a/C] mV' C; R>

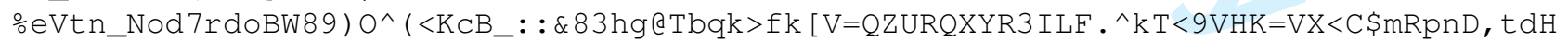
$\mathrm{P} \$ 8 \mathrm{Q} \#>$ ' Gq\$u' 'DYf; Z\% Sh

ㅇ [ ] " \&PHIj<W] ZqqJ>4\$O [M`-ZZT`n; ZF`NUCnI7Pn ( [ [ AOOk] X; G=]`TQHoG@U_-

OPBObr@Dfe ( 44 ebQcB<sR" ?3E"R\&u` $2 \& Z Z 8$

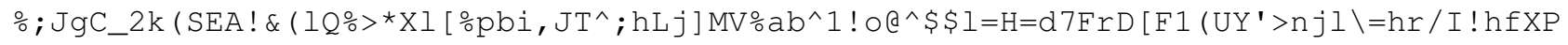
Zt1RI\$": ') S[_Oiok; (a

4 sm7\%W\9NiLdCja5L 7\#GH! 0977"-cnOd 顽

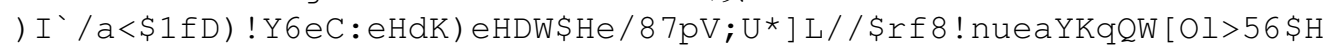

$\left.\circ K^{*} \backslash \mathrm{X}\right) 3-$

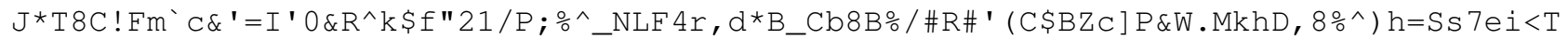
LXg50_C. 
gIhjj\&IA1g\] *5"TgYnb9PKmd; P=W"i [ "M3p62_I4?X.c>>ABfr36P7Frkd8\$-

jh! jsmNieG1tm5a\&PrmF 8SI\&E\&]NY:Y (.H ( t\&

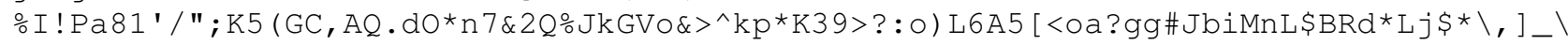
] DIpLeD^qX [D\# IW:

\%eX2GYq_: L_@6\#A \\$bb! k\$ / L3V"\&Pb-\&l?2YWC:^) hN*0Go-_D0UD8SVhY57/:12=T-

hfB6CqMOiAcs *2VG\%'fZOkC@\%BY\&3! K\

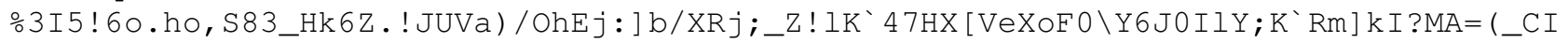
9E०.C\#Wj\&RmZFU6 C6VC"

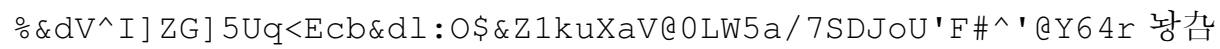

$=Q L k W r ` O, E=J t, ? @ \$ X]=]$ "X3MYQTpH_ctQn $9:$ hcp

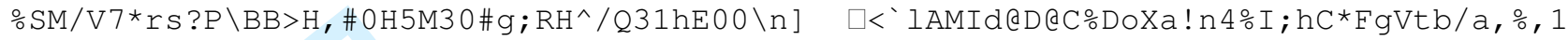
$\& \mathrm{~J}<\mathrm{Hq} 1 \mathrm{OuMg} \backslash \mathrm{OV}$

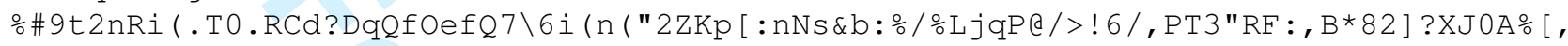
CG`SCiYa! (VAh\$ $<$ pN [m

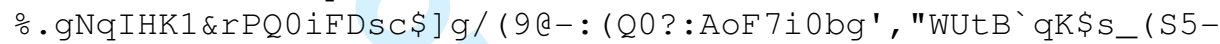

* / \$mC7, P>CfZXWjoh*M_HVA/ ], ' $\%$ E\#uHW7\&" :

\%goOktjjgqa*j\#`\$*Z; \#WOCV-

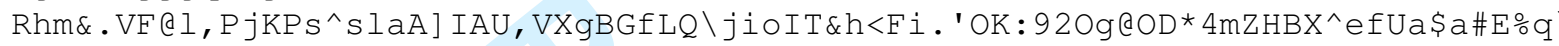

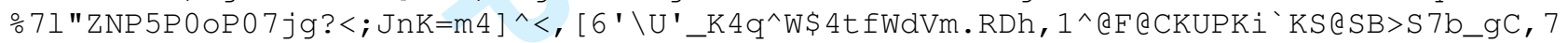
Jd'^Vja1;V $\square$ " Ih

"Hmu\$RVI..r"N : - [A ' Z ? 7c2Hrbr, KmRP5YV_dF' S ?0*.R5Tc1 (66"\#oobe\$ \a1g-

NTH9] h! \$J' (IID; In\&A0, >DDI^eKC [UXGL=

○YS ) j\Ja/_E-

$\mathrm{ab}=\mathrm{GKlQ}$ \$pH $(\$ \mathrm{kfL} \backslash(\mathrm{V} 61 \mathrm{a} \#$ \$KV, \$l b. K<5dMOKQ\$5Dp0; s\% ! 0Q1UKTB. HJ [ IS`UVd6ZN`\&kFZ, 'Qgrca9 $>2 e k A * \wedge G$

․ DuC\#) Blib9mW._B'DoF@dRbok 7ED=. (^adC_0aHS1@f31VCKJD<, q' "gG-

! US ! 1@i]9_8[Lp6>s3Q; II@CP': i\%:VjP^Z\%rCf

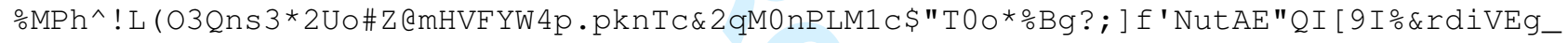
f?VMXrt: : IK\g

\%b21TN?nmg9bnVYr9q; ] Ms 8 ! QD31<ic $\square \& 7 \mathrm{~N} ; 2$ **d-

C>8i?BJn3' [?iRZKJ7'TJ.' *, /6QAUQUN4! > @noCjT7-Kd!) 4TPKWaS

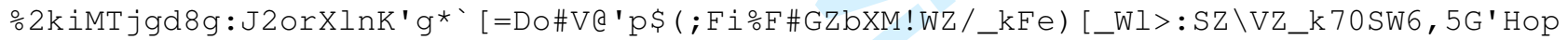
V4\#B9<e3RjqKc1! "! N ;

ㄱD요 [ ) TFHE"a, =<edMYeAim9"GH*NXBmhM3L8-

*Kp\#? [ s^9n?Vk?; ?W1S8 [t?GU\$5ORFI\$X?93VCCG9. 'V=Z_\p\$8\%M9UI0nTJ

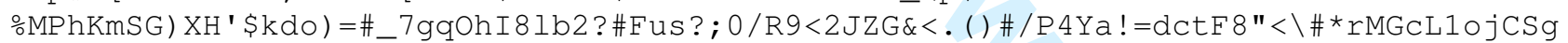
ILFE. "M [ $9 \mathrm{~L} \${ }^{`} \mathrm{a}$

으Ut>-

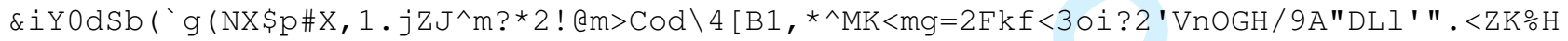
/p08e6_NQ1 =

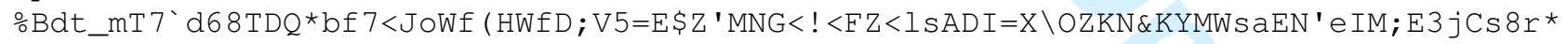
$\mathrm{d} 0 \mathrm{f} 7 \& \mathrm{BG} @ \mathrm{nG} \backslash(\mathrm{lkX} 4 . \mathrm{ZR}$

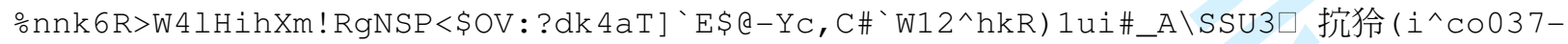

$08 \backslash W: j u F P \# \frac{\circ}{2} n G 0$

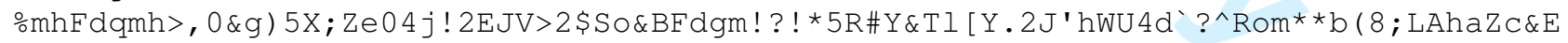
IAWKQL $<4 \mathrm{Ha}>\mathrm{LQ}$ I cXp\$XQK

을 1 EkHgk ( / Ss > LVNi *psM: qkTADn\#b3F SMYRRTpWWI-

bHk ) pQSWMASIRPAPeg2^FLYIp`3SDaD:RSBOne) t\&@1bT; *i'-b*EgSHn

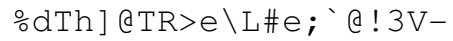

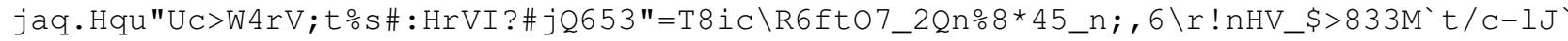

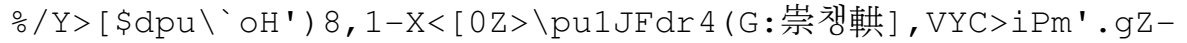

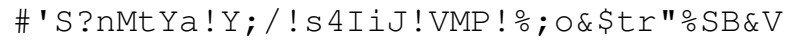

$\div 14 \mathrm{NVG}) \mathrm{g}^{\prime} 1 \mathrm{U} 46==\mathrm{aq} / \mathrm{aZq}=j 67-$

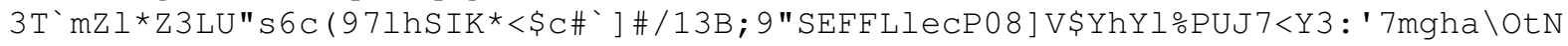

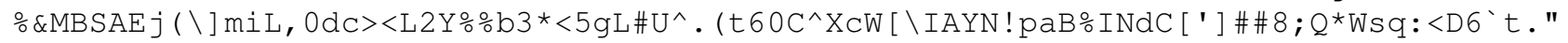
$\mathrm{p} 4 /$ VVe\$! jT8jQ/ :^:DJ, n

URL: http://mc.manuscriptcentral.com/tandf/tmph 
FXtLOYOXQ, 7dX0_P1pqmH! W=E / @OFD; a 5>C7H_9rrrf, /X5W<NhEO\&H3HR? "AY*hdCaN3 : L=7u ) =fm 4 i; $\mathrm{A}$ ? rdFm $=\mathrm{u}<\mathrm{A}^{`} \mathrm{Z3} ; \mathrm{6}^{\wedge} 4 \mathrm{ua}$ 


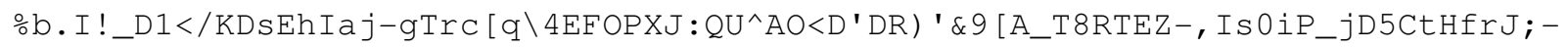
$\mathrm{V}<\mathrm{K}$.$] es 5 \mathrm{~d}, \mathrm{p} \& \mathrm{~W}^{\prime} \mathrm{jnf} 5 \mathrm{NE} 2 \mathrm{~B} @$

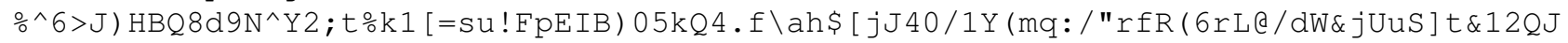

$\wedge *$ dcf $(5[\# Z B j .>E \circ t H t G$

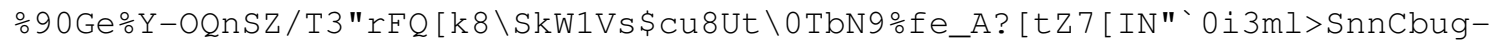
b 7H [>3D] W8-6\#; $\div \# 3 \mathrm{CCt}(\mathrm{nS} 3 \mathrm{nn} 2 \mathrm{XSI}$

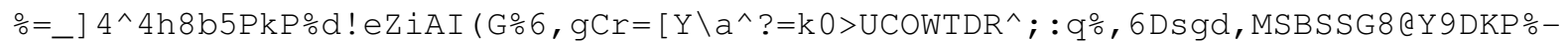

gFY\&pgu\`sB! ! gNoK

ㅇ $[\mathrm{J} ! \mathrm{I}-$

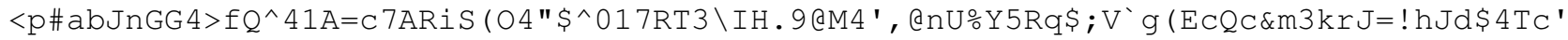

$\mathrm{SK}^{\wedge} \#$ \#_QUkF_Vj*]Q

X6EmYN ' QUYF 8t [AOA 4 \hJ7Deb\4JL=aRI8_GPTM9A-

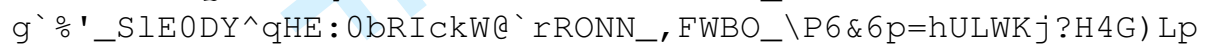

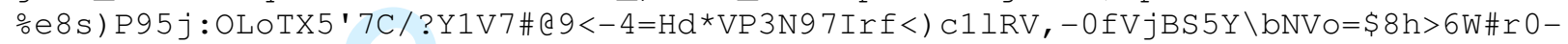

'p^d>rY2 $\mathrm{p}$ pol\$VgTblDDob"

$\circ \mathrm{C \#})=\mathrm{X}$ ?'WOX"FHO[n] GJ-

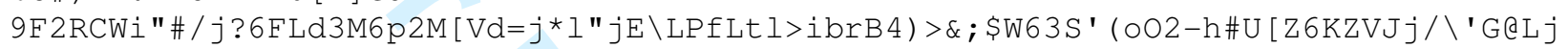

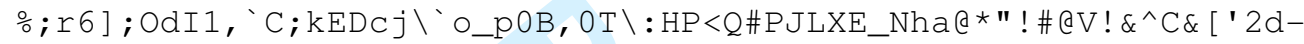

_m6* 'Qi\$rWBE!) TsGu:qe/!UIQ/\#IUI0cRjE

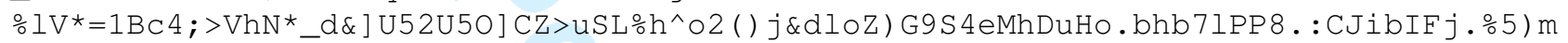
1.jFE4p [SHSDHu; $4 \mathrm{~T} 3 \mathrm{MEg}$

\% h"s?k23<6, MXpIVSO/O<, sgg] 3G8, mgPqKT?S3I "oePDCjXU (C\$X`51T, SgJKk; U⿳N:Ai ?PfQ\#PF<: [ , Z2 bW!n>Zi6 $\mathrm{d}^{\wedge} \mathrm{STdRD} 4 \mathrm{p} \backslash$

\%OI2 [u; =; \$R72Qt;ik^p!i!f1s0\$VR^s7poGd3Pm0W) 18uJ3, (2024'SP=g`+DUN_ea5em\; qs"t/tD( $2 \mathrm{TWQI}=\mathrm{YD} ! \mathrm{H} 4=$. ' $\mathrm{lrV}$

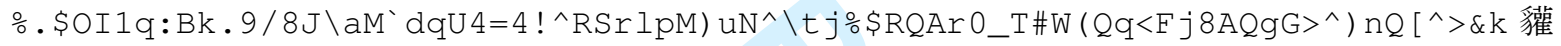

) ZNOb_U"R=4VeE/aB! $=\mathrm{pl}$

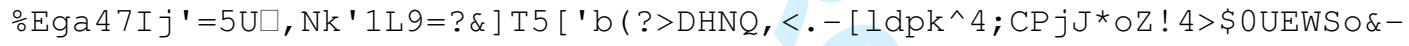

$+\& t Y) U[n P 4 H \# K " Z \backslash s d ; g j j U ?$ ? ( $\div 0 \mathrm{TL}$

\% SICA, f $\mathrm{f}=\mathrm{U}(7$ ? Bmg<] k"S, P"Z?kqi0:=[ . Ci-

$1 r ; ? 9) \mathrm{k}, \mathrm{C} 2 \mathrm{D} \div \mathrm{R}</$ ? iG8 7CLjRRFiDE=V, GE [Y_ECBAE; [K=CnH68M\&p"k\%kE9-\&

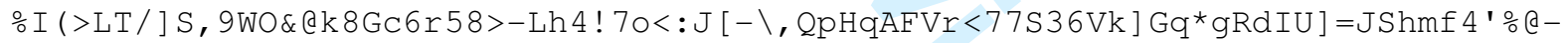

g] tjrjk4hYpdk3m>dRK] e-SV5O

G6; \n=_GG?\&80gJHj_s6-:Ug \102c.V9`6"<C 薈 $\backslash-$

$7 " / 3=29$ ?3) ZCF5NlD\&uW9fB4K;^] o_f89iC\#b*H:UD8 $\mathrm{g}$ *X1BFa>RJ4mY

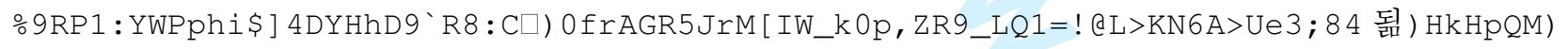

S6UW\$O.dai! ( @

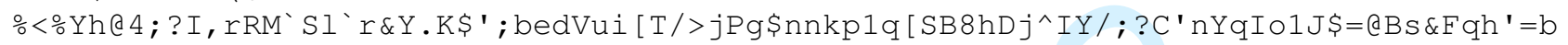
$8: \& W R R, K C U \$ j K G$

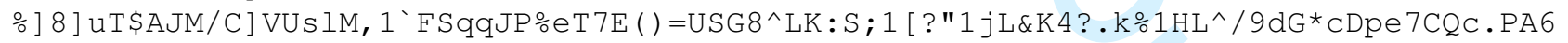
$\mathrm{dGn} 76 \mathrm{~T}-\mathrm{AdNr} 9 \mathrm{C} !^{\wedge} \mathrm{Tm}$

\% \SVO \snC.7*; O2fEkkFo3 (YYCE:O:3c?jb:n:) \%4\&-pUGVQ_/ @\#*, uWdqKC'_r-f>7YJn\$O-

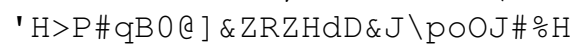

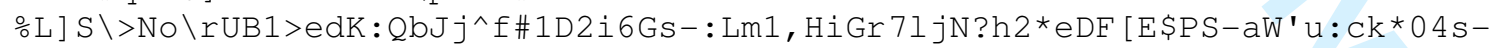

IeSQbeo ( ) fZ6) GiH; bQZVZr'/a3F^]

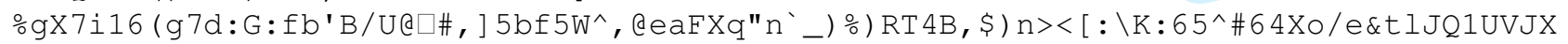
$g r *, ; P A=I 3 / Q \cdot * '$

○a9Wq9S: ! *, r\&3jr>Q0"6^M`d1iYm50rWdQC37) bETX6Ar/3; , V-

PqtAFG9\&@WN1; SttE2Q/Q, H 7! ?o*Lik! o52_U, , J; Lpk ! c

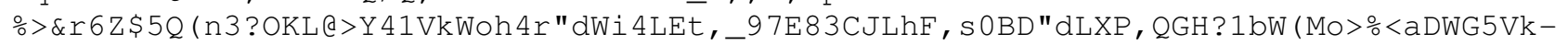
i-6AeZlff@Z:f.No*rOUn

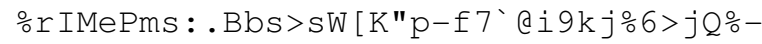

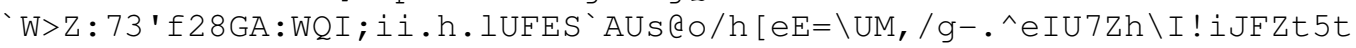

ㅇA $\& 9 \mathrm{~F} 4 \mathrm{hmJ}$ tcQbfTdU\#^ㅇ-

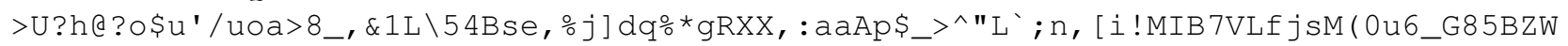

URL: http://mc.manuscriptcentral.com/tandf/tmph 


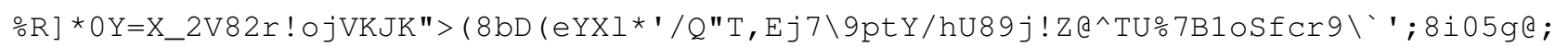
VO $41>$ MMPNI $\circ$ j' 4 Inp

○gq"9bGbmqUrV\#Ao-! pTOguQM' ‘ an@F_-FStq*s!3nX (sU6\#I\#-

C, BMme/AOHe<EO53 [QJO_, ) SQS^K@QB] CVil=M5Y-6" (mb7^14

$\therefore \mathrm{c}<\mathrm{Ji}=$ ?mR, (/i`BdH^uY8]) -

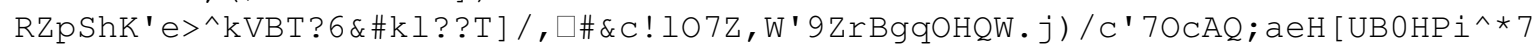

NVW` ( $\left.r Y O=I a F \backslash W C E @ 3 ' m V=\_r^{\wedge} k\right] s-$

$\& O n=\mathrm{CggW}) 4$ ! *'D; \#193uaMp^Ho=G) :<kPd1000`b!JkT\%=Z0TSV1_E\#55`W.YOfX<NT3>

DG>e\#AutfkCbE5," ( (: -

Jic [Z:2\!Yq.I"RCn, hV] f!RY8BTU1u^P3:h>:3) 0:5a_/6?gdVQ2ZoHApa)X] : :B-\%aBM?HC_l, h

○Q'/4pnDg5 \_X\&SR4_L:W: \$) 5=>a_V[-

I9Vkd6;Hkn] Jf [U.Eei $\backslash / 00<$ \&PAi ( \$_><QTP>EkI*eJX/ecZr528@u[K) 9 (r\#3IKoB

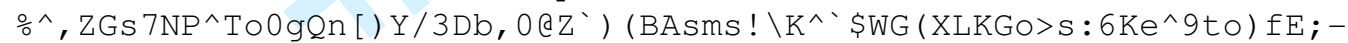

70'><1T4hX4S@pWZ<: IsQOKE' eOPtJeD60YVX

。 $\mathrm{e}$ imBY $6 \mathrm{Dkt}<\mathrm{Y}^{\wedge}>\mathrm{T} 5 * \mathrm{j}^{\prime}<\_3 \mathrm{X}-\mathrm{bXg}>6 \backslash \square$ 錹

*f], K9Y570=dYocLZ, ] \$1K8biM<\&E8CjoeB\%o6\$녕 $\left.\$ \mathrm{AH}^{\wedge} \mathrm{C}\right]$ ] 'EO=49decbIjG_

‥f! 8cf8E8t@Hq_V1neMXQC \dpVAW54a. ** ${ }_{-<e-}$

W7 naWqlH ! =RD" 6m9s >dL\&bVhsPu:porDSA ! 2OH<' Z\%e<cg\# 7aP79 [g0Sfp3`2Y

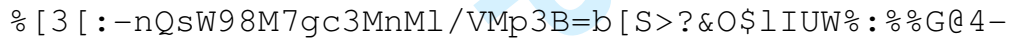

WtKle2NQek8W' (Ve'5./mpl\$! [GQnjeL_VQ>e*P[hhsANrVTSlf*\&

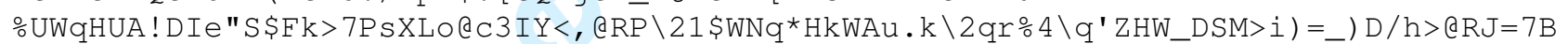
$<\mathrm{BCE}) \mathrm{P}(2 . \mathrm{F} \& 6 \mathrm{KQ} \times \mathrm{XO} \mathrm{KRO}$

F ! 8I 4 *YD3Iprdq<pR31 [] \&LNi^[nY>hsf4q-I^\&C.Ue-D7GnbLB8S5[^0;S_?kG=ZF>L/\#T)-

$\mathrm{fsZ}<\mathrm{V} 8<\mathrm{k}] \mathrm{uF} 6 / \mathrm{bH} 5)$. nP\#K! OjE"

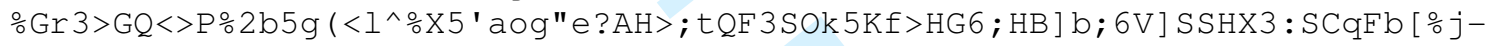

$\mathrm{Cg} 2 \mathrm{~T}() \mathrm{CE} \_2 * \mathrm{uW} ;$ ? $\left.1 \mathrm{WN} f->\mathrm{NM}\right] \mathrm{TC} / \mathrm{Y}$

○<4BN.<SB7>?Ynqq] So ) ' 92HRW) [C’ [ ( tgHdQeOT60K-0ALCMFe [; : >P^_L_A\#*_

XDR\#XEnOVS) 7nIV3; (pbSHVgY>aS 囃 $\backslash$

Ht?3.DKot’ 'Odr' [9\#DE4i9a\&j\%6_4rAd^iWF[R(?s] IK, H\$qNemn-

$F \backslash O @ W @ V 2 p 7 q \_1 j 5$ ?pdqH. ?VB>NE@\&\$` $h ; d^{\prime} X^{\prime} e K 1 S, N q C$.

。JtpQ (UtY`: ] u<M (cV7tWLPN`V\$=9\&S\Jfj_rlCC. (SC30<'\$-GmZ\#NT6, !>^?\$88; paV3 [-

$\mathrm{GOeC}=\mathrm{Yk}[=/ 5 \mathrm{kQDu}[3 @ 46 \mathrm{r} * \mathrm{~h} 4$ !

fm9L4Fn7"uP@ks_SqtsMC\#Dd^nO1_Q] s47) SVrK.7W-

?\#kIqCCVt $/=^{\wedge} \mathrm{UF} / \overline{B A} 2 \mathrm{k}^{\wedge} \mathrm{EHYHCI}$ ? >Wh9190Cl '=1c\$; [ ?^>0) ZM9VG [S5 [

○\$OSG.XDiT9Xo] ; =\%*K2T) ?C\#U, , 3H) dYHV`MU=。mM/\$ZkfV!FJebl?5?rNfIt4) 47, $6 \$ 6 \mathrm{JRLOT} 40 \mathrm{BB}$ ;0`DkuH2\$qd!X

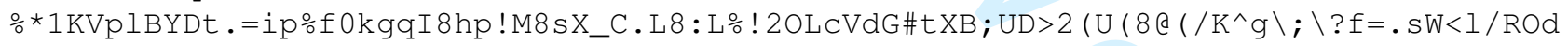
$\mathrm{VjU} ;=\mathrm{M} \backslash \ldots:\left(? 1 \mathrm{~T} .0 j 295_{-} !\right.$

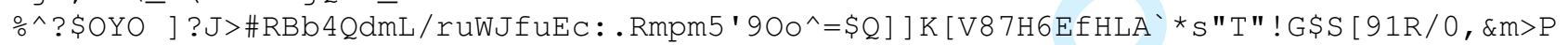
9fo) OT\&*J>\#PH. 6F

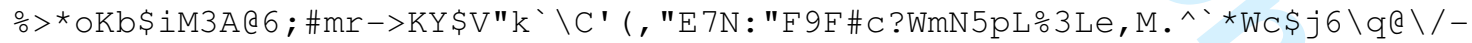

$\mathrm{Zd} ; \mathrm{E}>\mathrm{XRq} \& 9 \mathrm{bSWAmi} 1 \mathrm{fi}$ ibp5DqIW`H: $\mathrm{e}$

ㅇh $3 r D n]] J m T . C ` 64 L D) K \$ g f k h ; \# S * e F>(S 1 `$; WmDf ! -

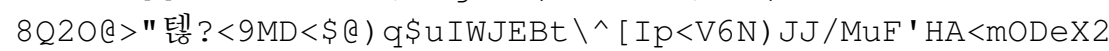

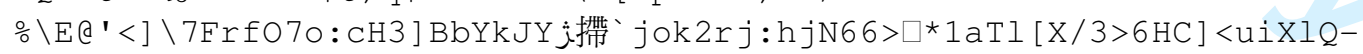

$\left.0 J)^{\wedge} 8 f h c\right] g E /(W Z 9 \wedge P F \# Y J f . q C K$

응 $\mathrm{ntBemCCQC} 01 \mathrm{qGBRQ3L}$ "Q\&ah85A-D8 "UG [6EN; XKZdkm9, pS . ekR=d-

$j \cdot g g S \_m 2 ` k \& \wedge=, D i E ; i T$ 홴 $=\& g q . Q^{`} H E a t D r$

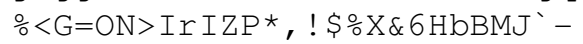

$\mathrm{fV}\left(\mathrm{IH} ; \mathrm{X}^{\wedge} \mathrm{e} 3 \mathrm{RC} \wedge\right.$ [RE@?YOJn*UdA121Kbs ' nGH/\#9Vr=E 's5Yu\#_C"V^<UVM0 ?TCpPi/NiJiRZR

。dNYgB; Wt $91>$ We $<$ ? YV $-q-3 * 7 D \circ @ A d O @ Y " * C " \# F 0 A C=n f T X E 5 J n>\#-\# U 8$ ! 0 ?-

:W' (aoUb`cd6G \njCcX\$O; Kcl $4 \mathrm{au}, \mathrm{tE}, \mathrm{kB}$. Jl

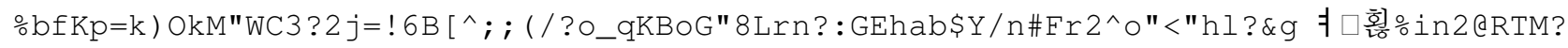
$E^{*} \operatorname{CuIN}-\& C l q ; \& g B 5 A$

URL: http://mc.manuscriptcentral.com/tandf/tmph 


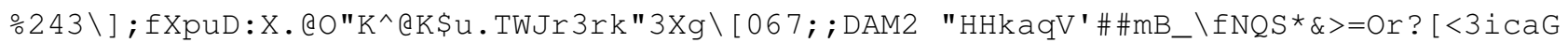
$" \mathrm{M} *\left(\mathrm{C} ? \mathrm{YH}^{\wedge} \cdot \mathrm{b} * ! \backslash ">\right.$

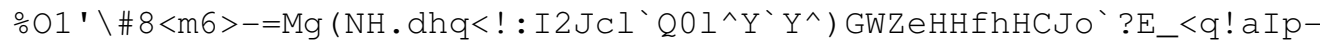

TU] A $>: S b Q H \# W \& V \backslash m$ ? ] c S . c ( H* / $68: \mathrm{E} 8 / \mathrm{K} \circ$

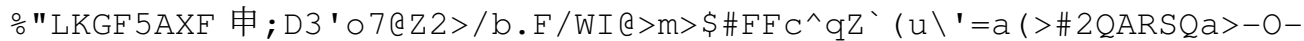

iO5\&;gDaY\$! .J7 JJFgh!'*4t/MoeEK2,

FR: 5XMpcgq4VIH! 3rVA: C : COQOFQuMq\$HUuQq7jg8. \$Qg-S@3EAD? 1 .>agn=. : K, BD ( -

Ggf $<6$ ) \#YPJt. f<r'. -hb] CR! $8 / \mathrm{S}, \mathrm{RsAl}$

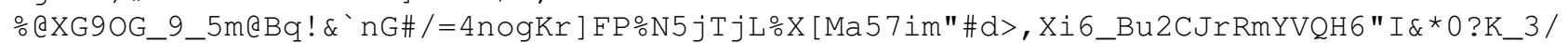

$18 \mathrm{JR} \cdot \mathrm{pKJ} 2 \mathrm{Ce} / \mathrm{IB} 6 \mathrm{~L} 2 \mathrm{Z}$

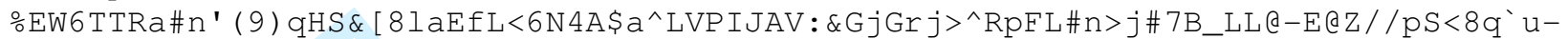

O6dn0\#k? [' (CnJrNRl' ;

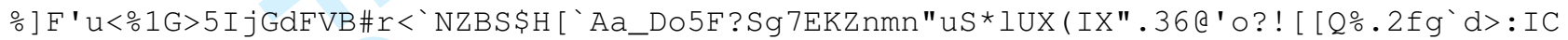
$73 \mathrm{D} 4 \div \mathrm{O}$ iD $>\mathrm{Us}-\mathrm{C} / \mathrm{nLRAg}=$

ㅇ, a ) CF16 ( $\mathrm{z}<<\mathrm{KRP} \$<\mathrm{k}-$

\&a38P0MmJGcd/Y ( i 6oYTm`hN8g4 I\$\#W20"m"N1G2k@m!X\$WWH; ] Y ( jbBf26Wdj-?m! Y@CeF, [?tQds<F 661j4V71YR_93*0608Zdb7r4; ] sJ; .g3SE' ' *f1gYZY;0iYM"\$m] 4aW1PK.W. *?:9n?W4bd\$M4GEi5) kpfQspZoN! 5FjsF5c5uWe

-uVAh6 Ka=I5q9RtN_RkFTq1OJ; I : aR28Mbj4u (1S: csb/WoJeed / (pmli\&TlF (G-m>3\&; afXb5re [8\#P"DgNUN`b\$Dca 7nKF`9

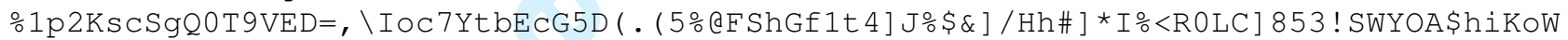
hou: ] $\$ \backslash \mathrm{F}\left[\mathrm{kL} \cdot \boldsymbol{-}^{\wedge} \mathrm{O} \% \mathrm{acL} 5\right.$

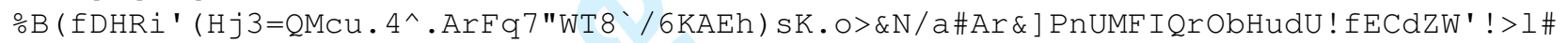
* $\& \mathrm{Q}-\mathrm{f} ; \mathrm{dj} \div 3$

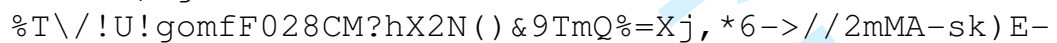

TlsmUrB_:AYBnEZZmIZY18g] /OUQ] OX3 ? 'Q@F ! =8dD1 * '<' ; 1 ! /

\% J J 2Km5 7BiW\$J\# [GN \\#=gQFCC69`j=-

SP8S*L2uhVAakF (MOKi1_IHgL\$ ] AB5DgYo]` @ ! \$g9J, tQ6Wa '! ! f " [ /WC\$ @CE4RM! Gq"r

$\circ 9 \mathrm{~s} .1 \mathrm{H}$ ?i-

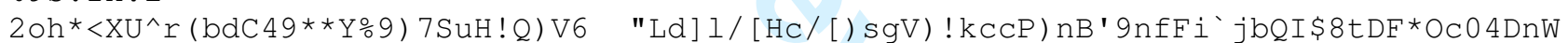
6 (XPYrR

YiN\$ $\backslash F>$, ) \#TAf@* [35;f'E) OK, : ) @K/0) G3 떦 $\square /$ ] OY*umk, F] jO20"-

f) iG:JDGsX'ZnpHE6AC: :B.KXkW>W] GQrtrg'

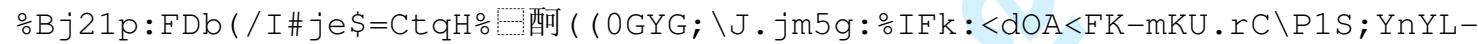

aW $[H W V, 0] N^{*}$ je\&uAWou5 (c8I?i)

W) Z5 YYrqA] ! b' ] 6_es) eXJ3SJr4B7oU>9YX (BjLG' ? YYu (a [, kOE>

O<u, VB\#eR7*?Yp\ZOqPLQUoj_*jo!so (RVS

MtT 7qO3GD*OgUUgHLSFpGi 4bnoE0a908-

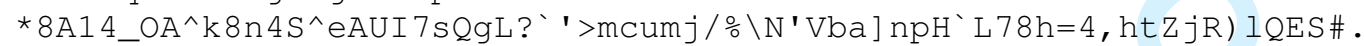

# Nnh (JJ] p 7W>, 21U/IXKf0H5M4DC`hE-;Fo: 7) VB.i/_5N\&D:p<eAr [ES-

z9^.njr, ? ) 9pCV9P (<k7`fout\&KKD61H=6E0ffAi

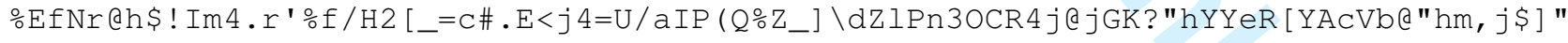
VRFA \UZO"ke\&Z5uUZST=

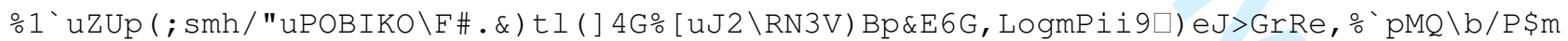
$\mathrm{LgStoP}=\mathrm{V}^{\prime} \frac{\circ}{0}=\# \mathrm{nFd}$

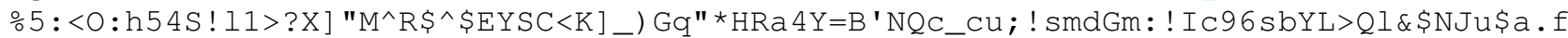
i。QE91) $\cdot j(42 j ; G B " *$

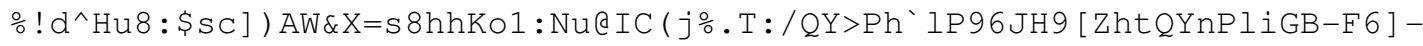

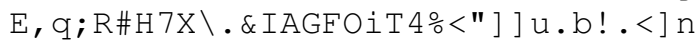

$\circ * 4>\mathrm{nA}: 7 \mathrm{GpcgF} \div 6 \mathrm{~N} \div ; 6 \mathrm{a} " \mathrm{pP} \%$ Afe9?dpq-

$\backslash A \div$ '5Dlo' 5OBMD jMdJ :R) DT"M7s \RRr?m (nb5G5Et\&<Q1h/UPdOI ; 8 iSnn ] @Zg

은 $: \mathrm{ZL}^{\wedge} \mathrm{K} 8>\mathrm{M}: 756 \mathrm{QS}-$

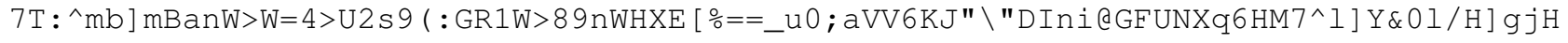
CX?

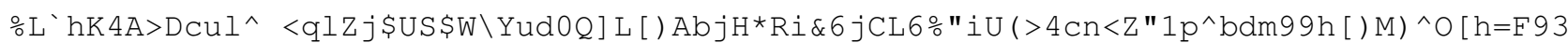
$\mathrm{Qfe}[\mathrm{a} \backslash, 7 \mathrm{nLA}>\backslash \mathrm{UY}[4$

URL: http://mc.manuscriptcentral.com/tandf/tmph 


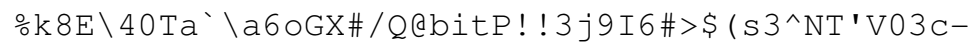
$\mathrm{B} ;]<\div$. J"t! / e 7?g"nPFQAjn\ $8<1 \wedge F r U \% H h 9$ \$U06AUGr-gXJ2X*"0pW

BZpL6PYdQ99tq8If\$rFLF_8qfQX:hbRE\#?q4lo\&1NQWIYS\#DI) gNe'_Vf_8Ybk\#b8bMD) G^E3seKr6h (K] T] SEK3`Si6BrUihA/

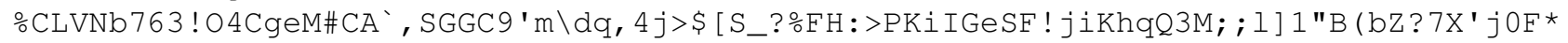
$\mathrm{L}, \mathrm{MO}$ "hnddClWf, $[; \mathrm{K}, \mathrm{pE}]$

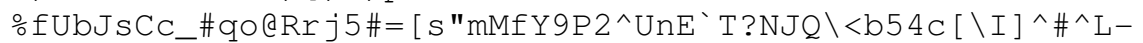
$=U k, C=* J E O Z$ ! "\#7P35Dk9H7R_\#L0OW3A\#Nn8LiWq"9B@Q

。Yn** $\mathrm{qBuY} \backslash>i] . e V W g R)$ 'WrR 嶢

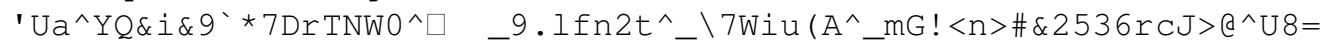
81^1'\&=1bqh"3_JQ:MZ30Rer=UI?288GNobU0M) h3-5.'AV 锍

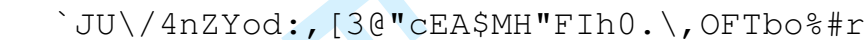

hfS_<<27T_fN8nOA. @MIpfeS/mg1 * KKi (YuaELB \\&l6um2befKCD; kNg/\#=*Q, KsLpu[tH?E/5>*WK ; qf@BND8V9>K8.Tb"\$?

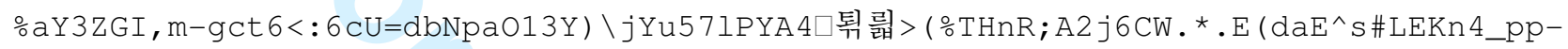
$\mathrm{XH}: \mathrm{S} !{ }^{\prime}-<\mathrm{hY} \# \mathrm{mT}$

일 3 DG"K-=,X], jF9N4df\$! q\$dQCrr]Yd*EhE`hlo3qKY-

e: $<\mathrm{d} ; \#$ Hq $={ }^{\star}$ egHm` $80 \backslash \mathrm{R} / \mathrm{T}^{\wedge} \mathrm{r}(\mathrm{XXjP}) 5 \mathrm{UfEl}{ }^{`} \mathrm{NCCjZTV71A \&} \mathrm{RgY} 1 \mathrm{P} 83 \mathrm{aK}$

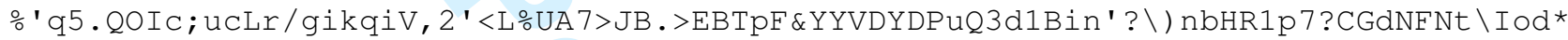
$:$, i. LaZ8S<Lㄷ'7A $8 Y$

$\therefore j$ ?XSUF $\backslash K-$

i 0 ; tc9q) Q9@. 8m( [rC8\$nk^0Q3BDH\#SH/<E; $1>$ : Gf / 0 ! tmV@ne"Jgt )*GqSn`QM?dg<V\&K* $\left.\square \_k Y i\right]$ b1h d-6pOaY

ㅇ, W5 jiBOY?] Q?_DBO/\$ [2mc7c.G\#Osm, \URmN"6*r; u 4 [ 8 ] Sm [ 3\&VA\&13Pl 4 ( Q8N2XPXM ( jHM\&k@Hp1 $Q \backslash .^{\wedge}$ gtoFFDJHTCp 7 btNA-

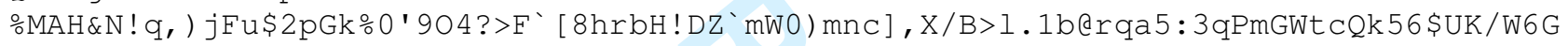
ta_0_\p"C []>.6SK"O5;

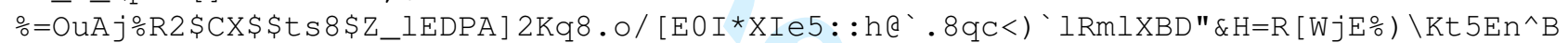
'R?d7YIUP] A` $\mathrm{qW}$ ? JmDd 7

。 [O8hQW) @M2SDY9=Dk.6R13.^p1G<J"Ec>u3P7W24`mm4hn+AbA! *i ' ; @_HIEmP) GePcji4mbd\&dr) $9 \$$ i3tA7AZY; Ijh2Y/ \Gi" "

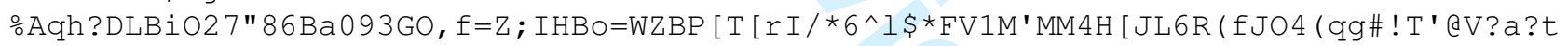
\#7?) B $\$ d$ Sm6sOalu6wUcjX

ㄷW=I5/NsE\#;/FLDq! $</ * i:<$ EMo>Ig0JQ ' i 7\%. 5<NiEpQ9Kq90 [T? (XrE! ] u6-

$\mathrm{NT}^{\prime} ; \mathrm{Ajq} ; I_{\mathrm{CFY}} \mathbf{\prime}^{\prime} 8\left[\mathrm{Mk} 3 \# ? \mathrm{O}^{\wedge} j \mathrm{kmf} 9 \mathrm{G}\right), Q / \mathrm{JbJ}$

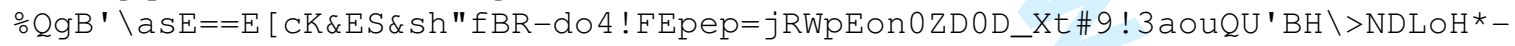
$\mathrm{KH}]\left[\mathrm{P} * \mathrm{VF}\left[\mathrm{OIUA}, \mathrm{fNu}: 1 \# \mathrm{HH} \mathrm{r} / \mathrm{d} 2^{\wedge} \mathrm{S}<\right.\right.$

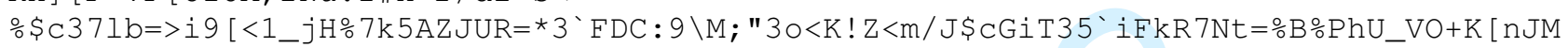
$9 \mathrm{C} ; \mathrm{CCRPOPOZ/rFjS,} \mathrm{QW}$

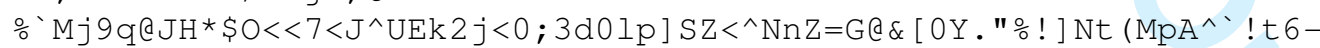
e: $:$ C $9 \mathrm{fCR},:{ }^{\star} \mathrm{Gh} 9[8 \mathrm{U}: \mathrm{F} ! \mathrm{C} 4 \mathrm{DD} \% \mathrm{qJA} 4 \mathrm{afF}->\operatorname{rogND}$

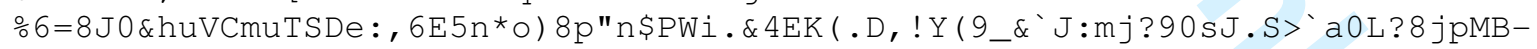
$=\mathrm{Z} 2 \mathrm{CR}) \mathrm{F} \backslash \mathrm{fFG} \& \mathrm{gb})[\mathrm{Q} \mathrm{C} \mathrm{CDqGZ} 9(\mathrm{l}[\mathrm{L}$

은 7 Me00^f, =-iXe@IlWY9sZ0K4\&Pr-\#/Xtgr'Jfe:5V4sriug9BY-

( 0 [We8 4nu\&McCq_b\&q\UpaAqGH) GsQ ' c . "WX\#NJ, uomPp $<Q$

BJnZr.-J5Ln"FCmHi_=Zc] $Q: 9 \mathrm{mEdJa} 65 \mathrm{u}]$ "Yp7c1AB_LYOU-

uo] ' aaY2O0B1nU=iABeT.H>kQ2IK ( V' $=$ MHO2@ ' WqU

D9Fad.!_L8_/ 99 ['! "b1-uFp-OAH?_oRN*3FqGd p5U68XeJ\$=\$i48e?G' JXXQKq, nX ( ( qX7"M$582 \mathrm{CX} \div \mathrm{f} " 9 \mathrm{dJ} 4.1] \mathrm{dD}>\mathrm{V}: \mathrm{OV}-\mathrm{A}$

X\#\#Fh】 \TOF! qFM*Mt 7\&Ij [, aNT\$Ge8 ["3roj, KP-

\#DI13s=N4H9 [_pVTB) ] R\$nbL) 5H3\&DEfNCOO"<lJb4 j>Eh*4'Q* ! $8 r \& b T$

AV0no/qXV\#4ESgG! UdT \X.4Pe55fL! ( ls [ fHW2S] $964 \mathrm{Gh}-$

W@Y] [\#/IO $\%$ pnG\#t/M_A! qJXa9u08Sj\&Y/D/<Uh [D;Z7X<\#OH"; 2

․ ; . I@je^5hPd @ $\mathrm{de}, \mathrm{FMp}[\mathrm{L} 4=/ \mathrm{J}$ ! br\# $=;$ ! -

E379mmg ] $>Q E$ : 9MD \Y] XSYQp61kD\&] 1C; 'Au`N), B\$OIBHaY3G*Os?QT*01ZHQX*, \&N '

URL: http://mc.manuscriptcentral.com/tandf/tmph 
G_mO5rBnKf*Q_pVcdCVd4J /P4L\#nirQ>QC3 ( I`sU<pu0'Y7;_5aL\%o1"sp. 1 ? @I1F8qDlFnVoqg"no! $\mathrm{C} * \bar{W}$;_De-OO KEp'"=] bIX

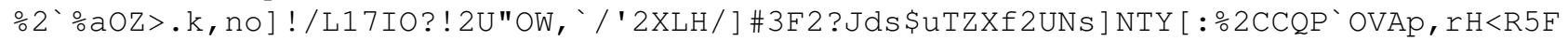
?>LO\JKtGK $1[1 \mathrm{E} /(12 \mathrm{C}<$

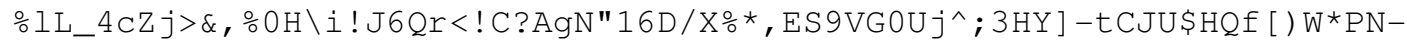

$\mathrm{N} \square>>^{`} \backslash 1^{\wedge} \circ ! \mathrm{AjX} \cdot \mathrm{X} / \mathrm{XYEmGhFIIB}=3$

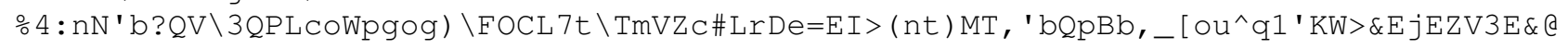

5C) o] -b1g9XgS 4 h@MR0\%

$\circ \mathrm{Bbm} "$ 七 ケ

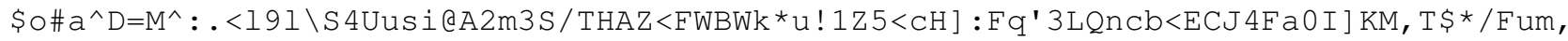
$\mathrm{h} /=\mathrm{a} 9 \cdot 6$

\%`Us ! ujJ2Md9Z4]_] PGsr1uR5BEtB_aC\&MWrCdGFOYl61dI * 8N<O" ! 9J1Ph4H0RW [rY<7] 1VdDd6 5Vq: AB1 ' / ] FAC\%kf015B?gLr

$\circ 5 \mathrm{KmN}>^{\wedge}: 1 \mathrm{~N} 5 \mathrm{Cu} !<\mathrm{T} ; \mathrm{G}-=, 18-$

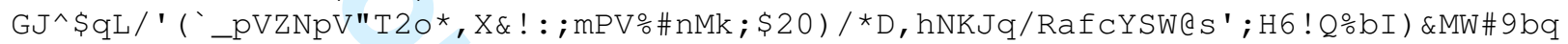

D \*9aU_dAo[8TM"gk_me?MT, aVBr\$; ) a \$XT80ju?_n:Ar حص顛*] Pd_tf9. YVIb\$B, 1 ! 瘅

QL $\left[\% \mathrm{E} 8 \mathrm{gF} \wedge \mathrm{nI} 3 ; \mathrm{aeUg} \backslash^{\wedge}\right.$ ? $\left(\mathrm{H}^{`}\right.$

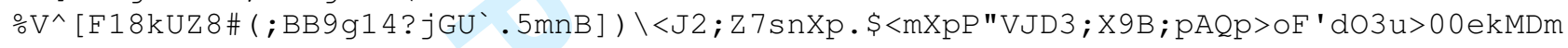
$\mathrm{M} \div \%$ TPHVOCT@X_F@ORd

$\circ \mathrm{O} ! \mathrm{K}(\mathrm{c}] \mathrm{FpH} / \mathrm{O}>\mathrm{Y}] \mathrm{aV}$ ! cP! HAs; JjPR3*NL-

$\mathrm{hA} \backslash 1 \mathrm{k}$ 'Wkdka/P<: 8\$n\&CYBfm/9JZ*ie1AH, s0H3Z] Dp\#J ( X16\%Y@u=bf : O 貽.>

H 7tb.WB! Q\$3F@bP9<*A?<HSFeCTHM?/q@! \_/2hhlEfUQmmaNcB6fizGIM\$s55/QP3=dOf (mc": i 7kg *bZV"E;B2; $97 \mathrm{X} 2$ ? Xc \eHR

$\div \&-$

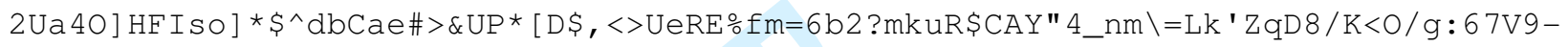

! 0$]$ QinN $4 d=<; P E$

ㅇ $] \mathrm{ZJ}=\mathrm{hYSUCBLI} 8 \mathrm{t}[\mathrm{edUYX} 4 \mathrm{LC} / 2 \mathrm{AAI} \mathrm{Q}-$

CkF 4/VMhO: \?L ! B6 4QTVX9`W4 ] *GR, L; Sh, IUO\#1? .\%, g, V\%G8s ( @ jXbeNZVTW3CA ( @

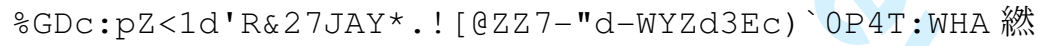

?A 똫!ZBJerZZKrNQdl 'WASP\$,S7kLajNQWZ_] : dcHCT/

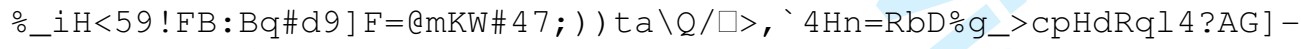

$6<\mathrm{T} 4$ gos 4N5 ] / uR?hZhk ) qT $\square:$ "hT :r !

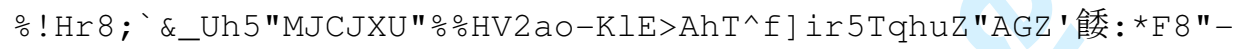

\$lqDE [V] BL <m [ "] 2 isDgaC3a=3] H4 [qEQCi $0<$ ? 4 ?

$\circ \mathrm{OE}: 3$ imKPW1 7H 4 brb' $\left.\mathrm{O}^{\prime}\right) \operatorname{Pr} \mathrm{AF} \backslash-$

" : 6M? jCO\&) n\]U6gr (NojY9`>pE"WIkfB'`"KSOZmAO. Gctd'jK>dP: aF. GC\$Y\$fkbA343;A

ㅇZYhHDr*LPVCC'j] rt5f4_Klj) f] Q; Q 7:AR74D=NQ, -

?' $\left[\mathrm{hn} \%<\mathrm{QsZe}\left(\left(\mathrm{KQIM}{ }^{*} \mathrm{~S}=\mathrm{H} f \mathrm{TqNJQstX0 \# )}\right.\right.\right.$ bQ\%3upgAffKI*POB

\%०/<^Bh?Q@6@8kB"bcqu- \sIEXOqKsWr] GYSP 'eXZSsM $\backslash e-$

Eps>' $\%(90 X 7 L L R n T(p<f[g Y 3 g ; 9 a j 6)$ qSY-"no`V-a_:H0i]\&MP : (,

$\div \mathrm{d}-$

m/ [Su [\##

L) $\div 3 ; \operatorname{TnW}, \mathrm{ngh}$

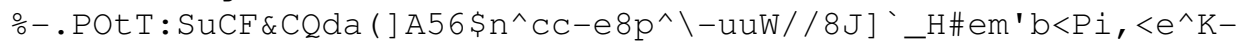

ODIA.C4 ] gYU) ‘*\#\$NY\&' ! OK9-93JO\#०(_tIXO5Q

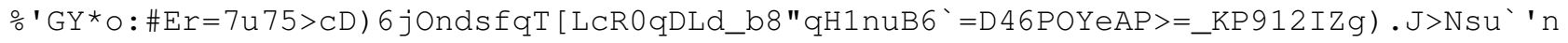
\% $\mathrm{j} \backslash \mathrm{H} 6 \mathrm{kYG} .: \mathrm{g} 1 \mathrm{X}^{\wedge} \mathrm{K} 7 \mathrm{~g} /$

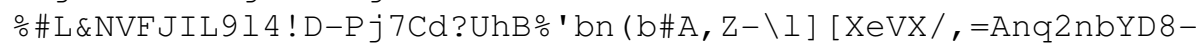

$\mathrm{V} \div \mathrm{NWO} \mathrm{O}^{*} \mathrm{BC} \div \mathrm{I}^{\prime} \mathrm{GaQVS}<\mathrm{R} 9 \mathrm{Id} \# \mathrm{I} @ \mathrm{~J} \& \mathrm{nI} \mathrm{jV} 9 \mathrm{f} \cdot \mathrm{U}[\mathrm{TKm}) \#$

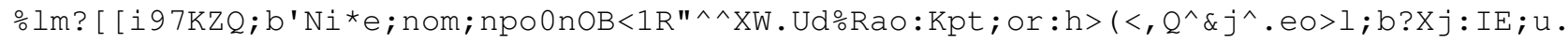
rs6 [YH (R., C, I I * bB) 7

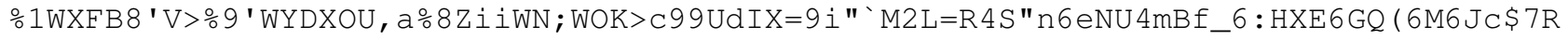

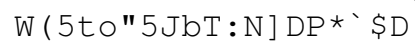

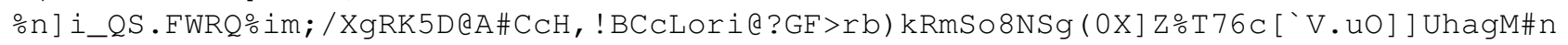
YPMVf", g [ athSWhJ 71, G;

URL: http://mc.manuscriptcentral.com/tandf/tmph 


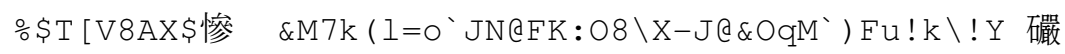
) 2fa\%A?IhD.>f7(^/JId0<@GNoW?] 5N08Y\V?a4g

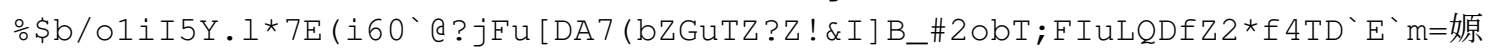
[_] $\mathrm{n} \& \mathrm{mT} \backslash * \mathrm{~V} 3 \mathrm{D}$ ? ${ }^{*} \mathrm{PXM}-j I_{-} \mathrm{a}>$

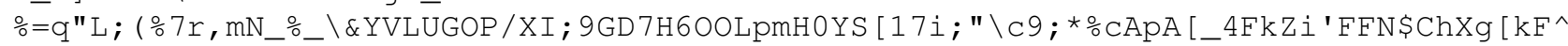
;GDa (\&iL.i77P8 $7^{\wedge} \mathrm{GWa} ; \mathrm{q}$ ○gUb\%< [ jutAT\&na3`0`Eep\$q=HctG"\#EA0kGG5 [0A? \#<druEQb; qd`Qb] mIZ-

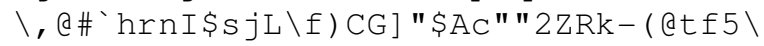
○Zi; rO/PlpD>m3a.5LVBn:MH3.gIM\#m81Zo@Mq6mJLgsG) UN9m; T) ItegbKGC) EHi00, "aPBLa] \B\%>! $\left.\left.\mathrm{tH} \mathrm{H}^{\prime}-\right) \mathrm{HB} \_\mathrm{OPYZ}=\right] \mathrm{OCH} \& 5 \mathrm{I}:$

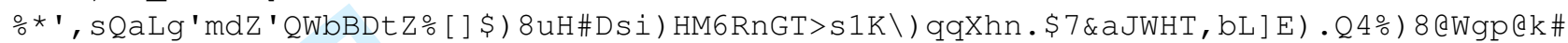
aJ90a.h $[!<$ Nh! @PZ: 8

。CPG ('V*?s*b7-t!; hf^。L4fH>p>33tZK! pnrE_bhL] 'XA "WUOE] OuOS ; ) [g89q*-$23 \mathrm{~s}: \mathrm{d}[$ ZAtrsg=92dgV83o-SfC1C6IDK

\%A, uoPN! 6Q"hjs) ! QCP! ( LC: ur-

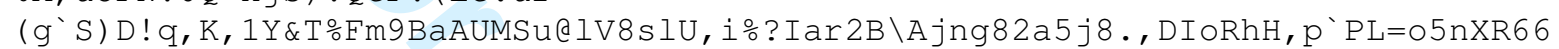

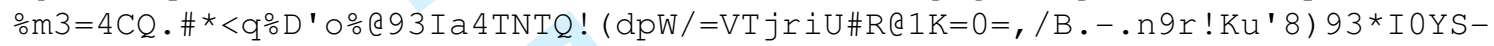
$3 \mathrm{Eln}, \operatorname{SnTCs} 8>1 \mathrm{ff} 7^{\circ} \mathrm{J} 2 "=\$$ ] HWgMo3V

○XCDZ ] \CPN! OW (XC2R=Z9j"U" (6B5GjO4U-tUHLP/) I50QWT\$>E8mEjO\#7GgG, QtSCGb] ] q: $1 *>$ ? ESCB, ? $\left.10 \mathrm{ckL}-^{-}\right]$f SC $4 \mathrm{M}$ ? Z $=\mathrm{W}<$ 5 R<mVi19\%RQ\%'Fr\&ip7j! elbqqUgd: (K<創<' $\left.Y 4>R^{\wedge}\right]$ f $\$ / N N Q$ $6 ! 69 \mathrm{Mk} \_g \mathrm{PO} * 3 \mathrm{aBP}[*: \mathrm{p} \backslash ; \mathrm{C} \% \mathrm{bcPVQ} / / \mathrm{P} \# \mathrm{VGO}$ (H3epn97dN

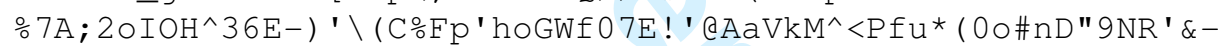
8nrYje) CXaR; UYFY, uNk\&CG\#Y \9D@ (Yme5_Y\@fo>1 ○Gpo:5p? ( "miV6Moo/ ( Tgp=s*a:0X\iKm4q\&fe\#OHPgI6AI [H,\#) gL\&0] T\# ( sO4 7UB $\square$.\&1@9"UGOQ\&, 3c49lLorE9"0) 5] 0s

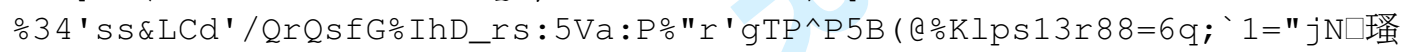
숏립! P4XU2F^"=@nL; NimfmBEUbo

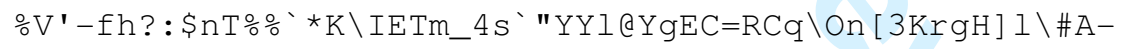
NWbHQY^3K^b._PBBDU; $P * d 6 D$, SShWeD3 Jn 'CDi<Y\#0=TV8Y

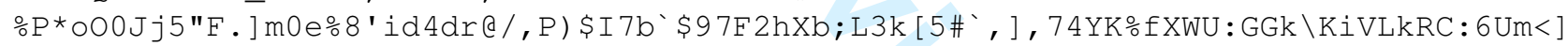
;Uff?kNV?YSqN_\&\%8gu 9

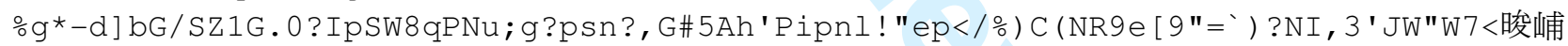
$\therefore, 7 \mathrm{bUd}: \mathrm{n} 4(\mathrm{\$ A})$

$\therefore \mathrm{KlRC3}-$

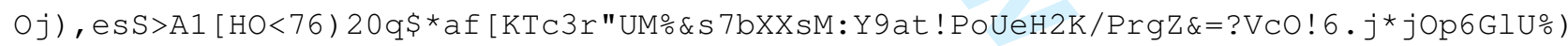
iL $\circ \mathrm{P} 11 \mathrm{dOgRm}$ ㅇoaP. -

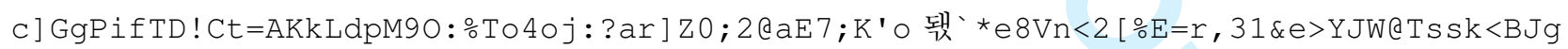
s6trNHWSLTD

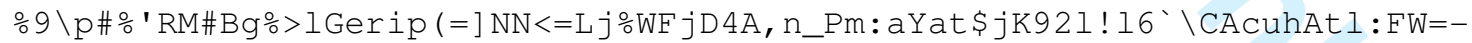
i\# eH<b9z.PH75 ?Hb 亘 $<$ ? $* 4 \backslash T \% \mathrm{mK}$ $\circ \mathrm{P} ' \mathrm{p} " \mathrm{~h} ; \ln \backslash \& . \mathrm{H} j 1, \mathrm{c} / \mathrm{)}):$, YIBRS, hfLR $<5 \mathrm{WOP} \&="-$ "?nG<U\& [\&OYQ: <J?_BT [edKH?UlSF [k=ka<q/GOqN\$) $1:$ ! g@F, OMJX\#6BF j

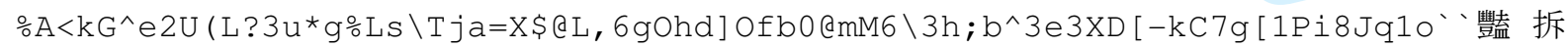
$\$ 2=d<m \cdot n l S \backslash 4 K$, 다 ( ) ";VGIbE > IY-

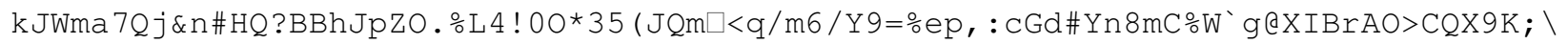
$\circ \mathrm{V} ;$ ! P (B) >nUqJ\&; 58G [UG+JC7IqB5V"C`e, b*LCZVQYdq9heKX\#LkE ! =D>tr1) -

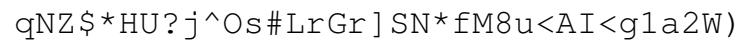
。*70d! 9UpqCdc/a\$'] I ( , Ul^X-ce (\$:f_D: 'Y*lRo*] =: $P * a q * 51 \mathrm{fWXI-}$ OTQqWK8*09 ( gt \$DkF\#P_, HeX 煲^SHTF`WZ*oMkk $\div 6$ 'Yf6_e1*h!M䁌; T); ‘ pQ5L]n?50$\mathrm{I} ! 2=. ; \mathrm{dt}:$ P3mq/A@haf!n) ; ?^Icl0^^0qM5"k/\$U\&XfN`5b<>_^k; , \$15@@fLBRXUr] Qh1 


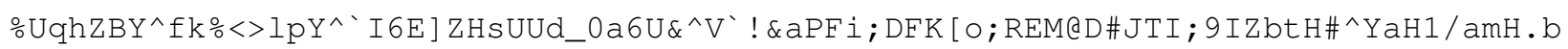
郅' [') G[337??\&CQ9Bi

rj:1Ydn5_'qQ \4Q1Y9L\&Ck12\%*Ke / [AYim\#q_@Vs/3R0A-

! X"Um</S.ndR\#OYY`I`r6! 87pH=qil<jE]0S. ${ }^{`} \mathrm{gHUSWHWS} 3 \mathrm{kr}$

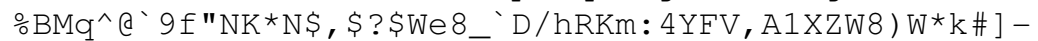

$\mathrm{CV}=\mathrm{hA}>$ ) iV! e) OI, *SNkrGTql=TX[X94RKng: ' 'ni_Wn6G'2NS

。aeVkeSmbG?Z08t; N (275gu] 0MOe \$OPY\# j<O\$q=kLU1DRR2S 7! @3A4oLZp^. U<f=tWHH\&k [ ]_RV! *O_u ;_9u0 $>$ MeD2/[rrKXf [

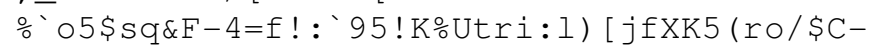

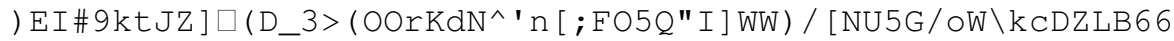

Zi $8<$ J2shicLRp1k<otc\%LaF) e. 'gZ<km7B<LAG7Urb\#7-

$\mathrm{n}>$ : (KH\&alpRD*8hR0fbS*V4; 3 f\% $=X s @ Q \_p 3 p / W " M \& 4 K 8 a \% 2=d>X ! 3$

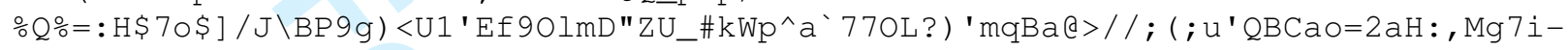

6) $\mathrm{kJLXO}$ J $=0 f 9 \mathrm{~L} \backslash \mathrm{YQ} \& 5 \mathrm{gO}<\mathrm{NN}$

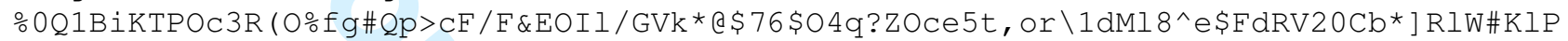
OYoq`) ; XZA, ?Gm.J`P51C

$\div 5] \mathrm{Ba} \cdot \mathrm{Pk} \$-\mathrm{Sj} 7-\mathrm{Pd} 8$ ? WJ^H

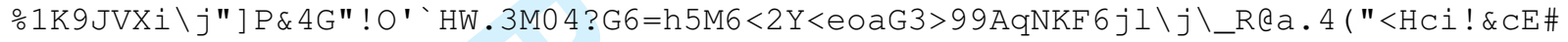

$\frac{\circ}{\circ}=\backslash((-$

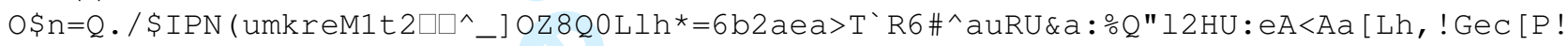
qks 閏

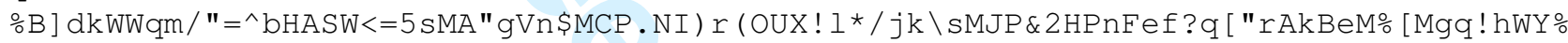

9 aL $1: e l>p(F e 9 \# k$

○IMi3as'X[6-

8g\#>:^cnZ>M2UfeNP1ki^LUJ6B) (5s.Rpcd]WQ9k:) @SN4fsh [a6iZNrRqaj;nFo/IJ>.i] 'g6^'M8f?

SfICsNZQ

○am`P: ] qOJJQgDg`] ) $8 / 5:-0 \mathrm{dkB}-$

\$/Bd \eFVTMeJ>odtOd:RCU\&pd_PAYm10\$KS\#Bq/MqfaO\&Y) @UP 4U8IWE56SXUd) 'AVj)

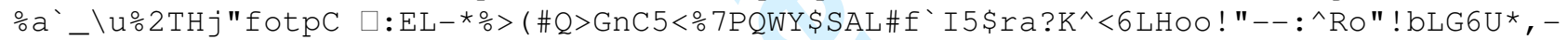
$m \$ K l e-M \backslash Q<L l c$, ?\#

응 $\& 3, * \operatorname{SPPZ} 1 \mathrm{FC} \$ \mathrm{JKq} "=\mathrm{d}<$ 杒

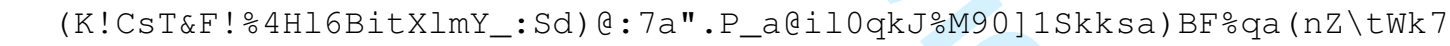

응 InJ2J'! \&\#7iWo\%P'9tOGldjYHX.n_.gO2-_. ;^Ya-

${ }^{\prime} \mathrm{Co} 4 \mathrm{D} 2 \mathrm{~N} .\left(\right.$, R9Mo*Oq/OqQ2 (Xg*, $\mathrm{HgCC} 8 \mathrm{q}^{\star \wedge}[3 \overline{6} \mathrm{YK}<1 \mathrm{LWW}$ !) $=\mathrm{NIt} \circ] \mathrm{RkHO}$

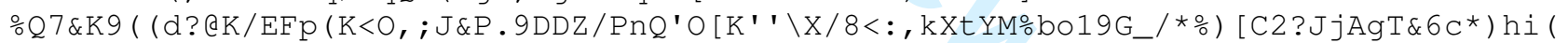
] \#=i\#r8 (t\$nsXh`"YRi

oYWd) d:V=; g1qCQLDJY*! *P9A2Pe] \#XA 'WG9Uj8A4i.\$ \Cq(E5DO_1a91Y<K, : aNCInub_LNeb ( $7 \& n f d$ $\mathrm{A}^{\wedge} \wedge 22 \mathrm{U} 8 \mathrm{mOWC} ; \& \mathrm{Se}(\mathrm{U}=$

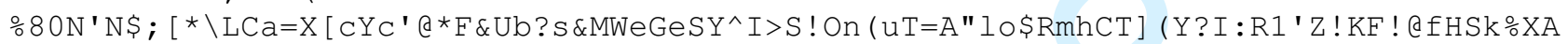
$4 \wedge \wedge \mathrm{nM}) \mathrm{CD} \div \mathrm{EQ} \wedge / / 90$

$\circ 2 \mathrm{gg} \backslash ? \mathrm{~h} . / \circ \mathrm{V}<? \mathrm{f}-$

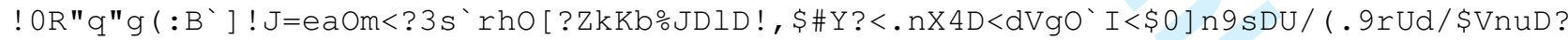
a $/ \circ \mathrm{A} 2 *$

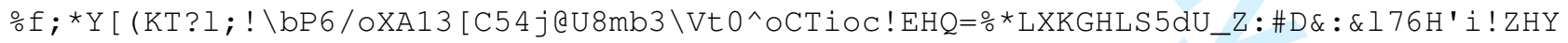
$\left(<, 5 \mathrm{CNCJVX} \% 1 \mathrm{pG}^{\wedge}\left(\_\mathrm{pS} \mathrm{S}^{`}\right.\right.$

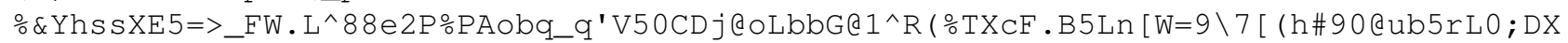
"1:dORU3PHa. RQ7

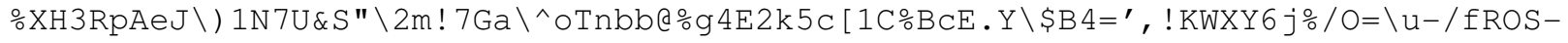

$\mathrm{E}>\mathrm{]}-/{ }^{*} \mathrm{VP} \# ;{ }^{\star} \mathrm{QZ}$. ${ }^{\star} \mathrm{tDr}$

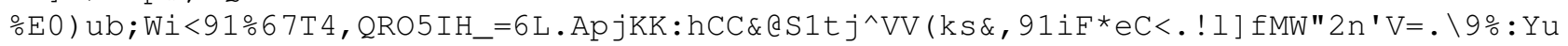
DDO'iC; 4BV"-1\&CZ1p-

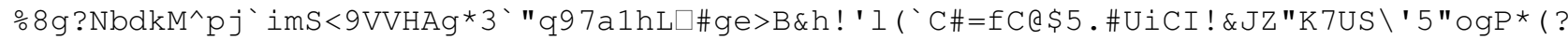
$\mathrm{CJ} / / \mathrm{h} 5 \mathrm{u} \$ 5 \mathrm{j}$ ?r/Y608S

$\circ<) 2 \mathrm{M}-6$ ? YV6P, " $>\mathrm{MQD}: \mathrm{NUpu}=\circ \mathrm{AN}) \backslash \mathrm{Zi} 03 \% \circ \mathrm{q} 0 \mathrm{~S} ; \backslash \mathrm{qBQ}=, \mathrm{OGU}) \mathrm{S}-$

;HJc50'riP/"S_q.sOj1 !!_ND5S>K-'\$\$2:IIjs:q\*Q4; I Wn

URL: http://mc.manuscriptcentral.com/tandf/tmph 


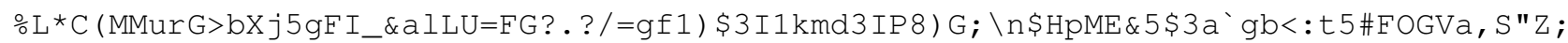
$<\mathrm{C} \& \mathrm{~S}) \mathrm{LCJN} " ? \mathrm{Ik} \wedge \backslash \mathrm{Bpmo}$

$\therefore 5=, j\left(K ! L \_X X f Y ! C l j 5:-i f>>b F 770 n=03 m t-\right.$

2J@3Q" \S9O[H5\$[ [\%=J.FeGA'PGQ; js"Oj'D\&Z@Q\$SG (e2O\&)C3HQ\%Z^وgsCCNL_i

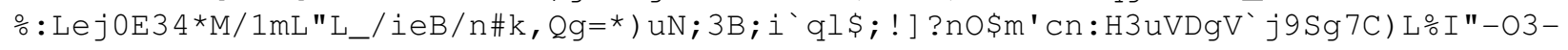
A $5 \mathrm{~s} \$ \mathrm{~K}-\mathrm{ThT} \$ \mathrm{SV}$ ! $04^{\wedge} \mathrm{H} 4 \mathrm{mKL}: \mathrm{h}$

k / t 85 "V@pRO-

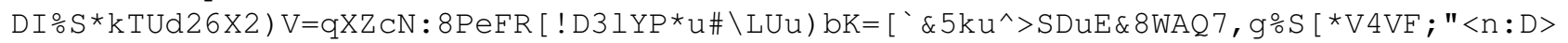
]

。QpP3k-='f\2FBH`oqWE1H, , fOLtDW5V\$H`N-

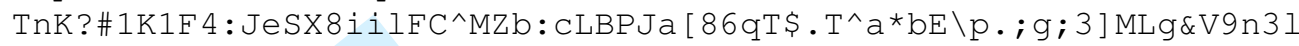

$\circ_{\circ}^{\prime} ; '$ ! fD! r $<503$ ' 4nJj- 'VO [ ) CKrrPqpp:-

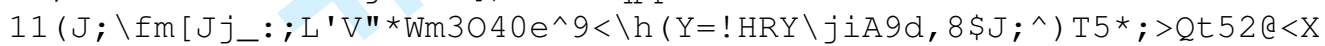

$\because 6 \mathrm{qNRG} \backslash \$=j \mathrm{Z}: \_\mathrm{n}<0 \mathrm{~F} / \mathrm{RfCb} . \mathrm{s} 4 \mathrm{D}=. \backslash \mathrm{hE}-$

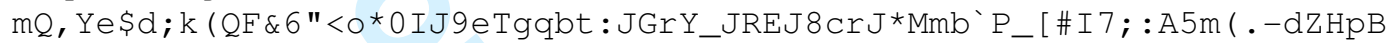

으IOQ==AJ?' 26E3Sd)I2, \j2八: $:$ T?k5IZ7-\#l*?>qC8h*ae*WHEN\#-

ONG7K, Zj`P (=pO^\%t_"MQgu' ! ! EEFE $\% \mathrm{~W} \cdot \mathrm{bFP} \div 6 \mathrm{~d}=\mathrm{E} 9$ ' N

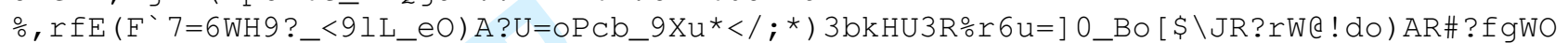
NAd"?Khdf->=_tp:?

$\therefore$ \# $\mathrm{TV}<;=,<\mathrm{tRON} 8 * \mathrm{~b}) \mathrm{a}-$

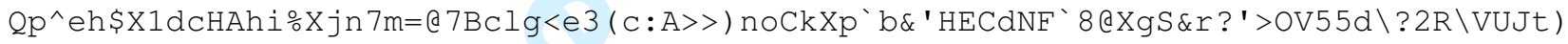

ㅇ.WpedEOppU 7; \k-BVe (咔

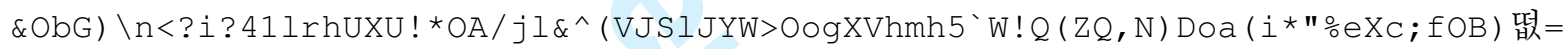

$\circ E<\# T \cdot 3 b T \backslash 0 @ 8 @ 03 e ?$ it $\%$ L $20 \$ / C l] u 8 K) 8] a \backslash 1 N d O o R \$ ?-$

$\mathrm{Vb}$ 'ndp 4 (d5EV=i。m [=3BDA`\#.370013\$_u65.=.b*8HW7V/GKM (26J

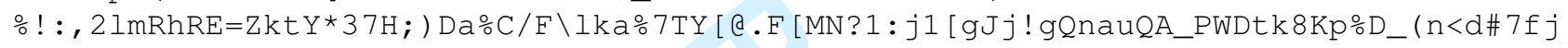
ICUX0OGL7DIGda3I, k/

F (_I’'\&6BL["^HpJUE8aKs; 9pVuf!,AK_6*265?g.FlRD ?u\$!\&'B-

TWUI, qCFdc \&N; ] Vin6X<B@ $0 \mathrm{e}-, \mathrm{g} \$ 794 \mathrm{GX}[\mathrm{M} 9 \mathrm{~S} 6$ ! ! R

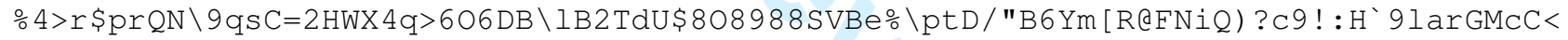
$\mathrm{L} 91=\mathrm{ukM}^{\wedge} \mathrm{MJ}$ ifXd0<:3C3/

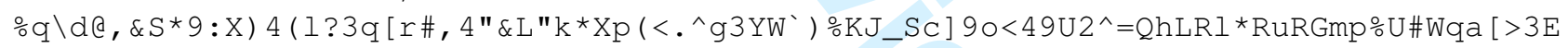
ee $\backslash J$ phPmK $\backslash * 1-K ? f^{\prime} 8 \mathrm{u}$

○U0CCa.D =B [q\#bW\&H, \#HD; ' 35MUGsulmHFr@P1Pgi. (>Hp_TIY] UN\$H7^0PWJa/=0dseo3\&@MGj8F) 7A $6<\mathrm{kTH} 0 \mathrm{eo}$

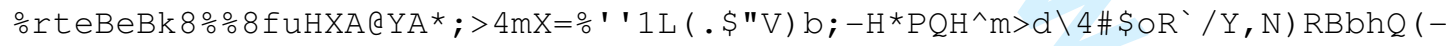

.$<j$ !YdCE $\circ W O N \$$ tX [ $\left.{ }^{\prime} C C I\right] 6 r '$

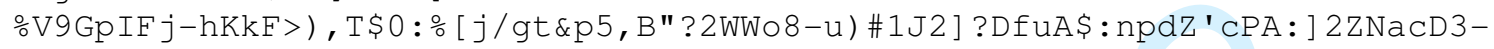

aNX>C $>:$ ID $>$ fnf $\backslash 7 " g g c .5 D \$\left(1 ?^{\wedge} \$ 6\right.$

이 ! ?.6n2'; ' (HP*\#*\$08r.R]*Fmh/\&Zn"CLT/*^?०(Qi-

*JUZZq@] 4SZ.r) M \p30J6ANSSO<k/Wg;9j-OZQ"K`N] b\&7K; 7 ;CS\%d

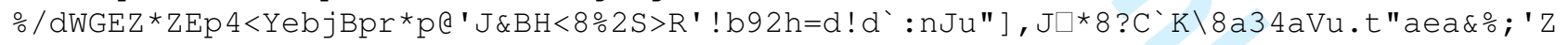
$\left.\left.\mathrm{EE} 9, \mathrm{~V}] * ! \mathrm{m}^{*}=,\right) ! \mathrm{u} \&\right]$

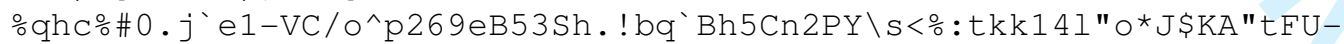

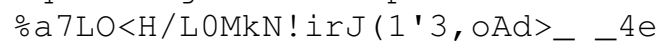

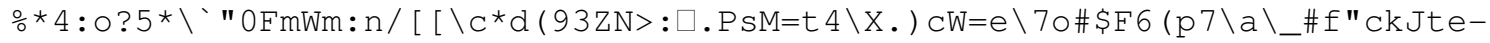

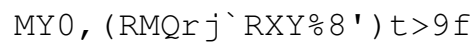

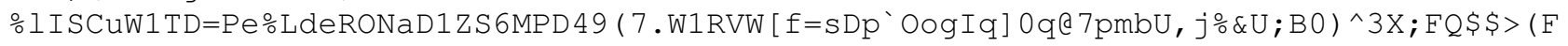
ek $=f H \# C^{\prime} \cdot b P H G e i \& V a U 1 O($

4 432G'*D<D\#'G:n! 5/AtV8VFQIP\$:R2HOAO2nlBadaZ-q: /A" OOHmB-

62], $A D=\& r, ?]$ ? >WfVBUM5019FXdD; $b=. \_d f$ ! BNd8t / $0-$

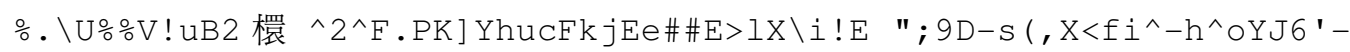

$B \& \$^{\wedge} @, j p Z F / F C L R h q C Z f Q U-O ` H 4$ ?

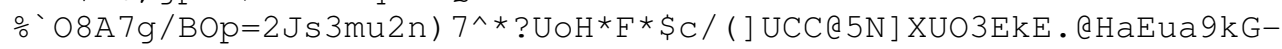

llN1i=N>5QJRVC5UFlejf_3<q/^p"k\&i.A\#f

URL: http://mc.manuscriptcentral.com/tandf/tmph 


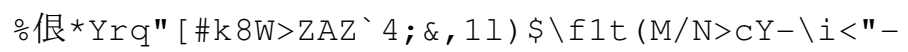

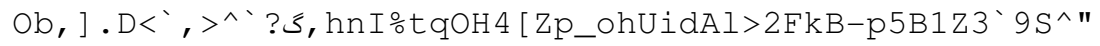

\%; ; Ut>4LU1Wda0kNYS ( ! ZB, \#MFIq?9K-

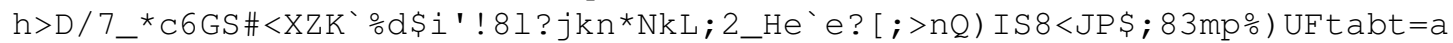

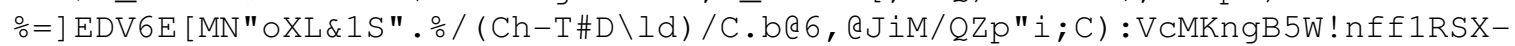

diY (Q0O [RP) 277R40NsU_@E 7Z] PYt

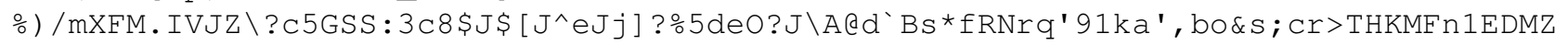
$\& 83 \mathrm{CJaB} 5 \mathrm{CH}, \mathrm{tn} ; 8 ` 3 \mathrm{~L}$

ㄴ $\backslash 0 \mathrm{CCQPTr} \& ! 7 \mathrm{ZBe}<\mathrm{RPP} * \operatorname{VDM} ; 8 \mathrm{a}-$

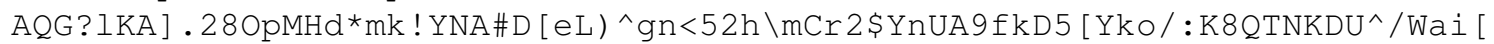

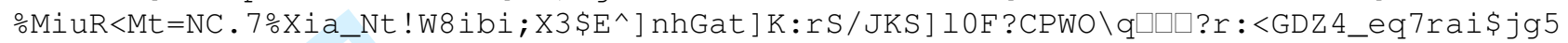
$\mathrm{FQJ} 24 * \mathrm{UGO} ; 2 \mathrm{~J}$

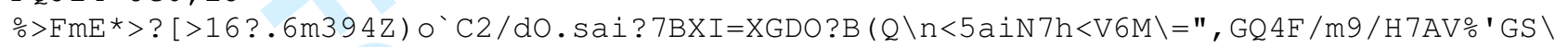
I"*W's] $9 \mathrm{Y} ! \mathrm{L} \mathrm{L}_{\mathrm{Q}}-\mathrm{J}<\mathrm{aj} \mathrm{C}_{-}($

ㅇ ! \#OF'k"Qdb] SUsc? I) EqEB/TnJDfZOOW=L--S [Wcm\#Q 惁

$\square$ 쌯 \#MqLS35G`1L@/L6=[(MF ] TCZYK2;n [^V<] j]) $2 \mathrm{Ni} 8<\mathrm{pd} \$ 9 \mathrm{R}$

$\div 9 \backslash \mathrm{T}-$

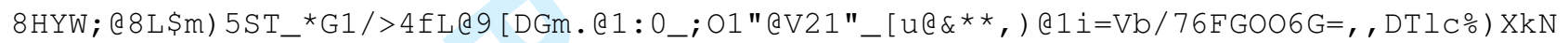
I3_] ??brj>WYYiD

\%i/Cn5, tFn8:3j*i*(bc>\#76gFgkr\&<JoIFGG ( \[M61<e!BNgbKS<Uh7[j1))_jD/>88eaNc6K9i1] > $O O j W D G * B Z b 7^{\prime}$ A_b's

$\circ \mathrm{Qgb}$. un-

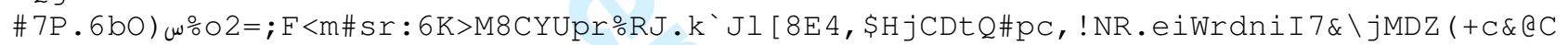
$\mathrm{KZ} 6 \& 5 \mathrm{Q} / \mathrm{FY}$

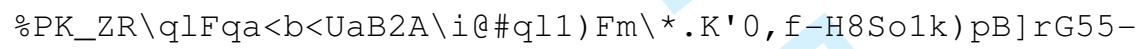

$\mathrm{U} \$, / \mathrm{P} 5 \mathrm{LW} ") \mathrm{k} / \mathrm{f} 3 \mathrm{~b} 7 \mathrm{AcGLO} 2 \mathrm{a}[\mathrm{jNV} !=. \& \mathrm{QQh}[? \mathrm{Kq}(\mathrm{bFS}=\mathrm{ON}=$

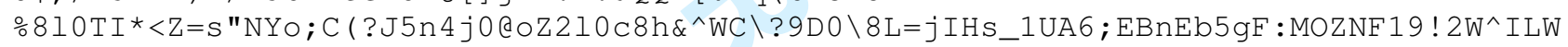
$\$ 0 \& 8 \mathrm{~d} \$ \mathrm{C}^{\prime} \mathrm{V}=\mathrm{EZ} \$ \mathrm{USAFn}$

GJ]Fb\&<T7_PI (Uo\%]M. \$, hS*O \HMI<1^4i[RZ3c/\$NWr^0;XB6j:-

N3, =l ?*`" ] Ri7Zi2fAQ; oYe*3\&7Tk] !hQin6.7iFQF.uB (

ㅇqmOG'Zf: ] ctcrj6?YLa) JjqEiQS2i.<X:M. 3bDnBY\#8"50T=@u_] ?, t^U\$eDWbH\$nM5_Mn?; Zho2LG Pt0dV1 7-;PdI/QV, e@

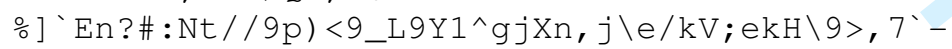

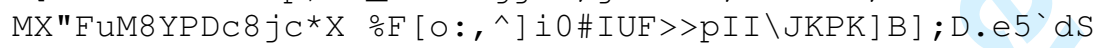

ㄴ ] $\mathrm{mTVk} 0 \mathrm{~S} \& \mathrm{Q} \& \# \mathrm{D}[\mathrm{t} 4 \& \mathrm{ad} 9 \backslash \mathrm{mN} 9 \& \mathrm{~J} 0 \%$; -

。* $\left.\mathrm{q}^{*} \wedge^{\prime} .=\mathrm{fOmf} 8: \mathrm{uG}, ? ! \mathrm{eD} .=.7 \mathrm{~L} ! 3 \mathrm{pNjO} 4 \backslash \mathrm{aL} 4 \mathrm{XQ}\right][$ [\#2 [X1i:bF.qq) LH=/B 聁

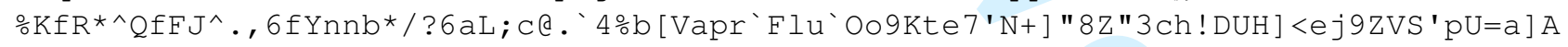
$\$ u^{\prime}$ ' ' $\wedge 94 \mathrm{G} \# \mathrm{~T}>\mathrm{J} 1^{`} \mathrm{G}$

\%, \#Ulf 72Q\#I/B\%h>l\%G (>, 'AM_gIa2-Z>gkk \.X>sn: . 7JWBeFRb3TE\%O[W>_=q! ip/gTgek; p4TVA`MY2t3_faa) Nu 嶷\$M5

? ] ; c?: G9>_2P $)^{\wedge} \mathrm{X}>\mathrm{UMA}\left(\mathrm{QK} / \mathrm{T} . \mathrm{O}_{-} ; \mathrm{Q} 2 \mathrm{Y}^{\wedge} \mathrm{tD}: 20 \mathrm{ni} 31 \mathrm{C} \% \mathrm{mB} 810 \mathrm{MSL} ;=\right.$ " $-\mathrm{fCQRM}(\mathrm{kNu}[-$

$60 ! \mathrm{Ed} \cdot \mathrm{P}^{\wedge} \mathrm{P} * \frac{\circ}{\mathrm{O}} \mathrm{D} \$ \mathrm{dmM} \cdot \mathrm{LnUUDP} 7-\mathrm{Y} 9 \mathrm{C}$

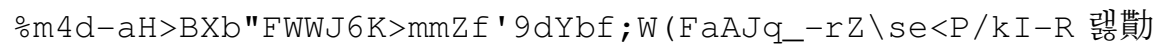

, 1 t; bS ( "_fBp4JcfkHA">=f0m/2=n1- '/c) Lt $\backslash * \mathrm{~T}$

ㄴ. 9 "Kr' [<\$lag\#mM87@. 7m) : 2s\&EP/>2H*bB (_es1AN6<X1H@nUmZaVr \$1O, j-rI! QZHsT) pi 3 "=0; [d 镣; ‘bV6 ( : GAtA $=$ GB2N

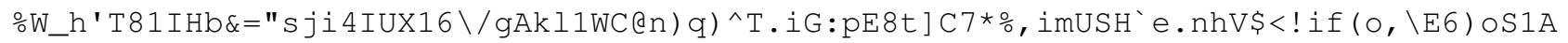
$.2 \mathrm{k} \div 6 \mathrm{Ane} 3=\mathrm{W}=\mathrm{VMHXn} 7 \mathrm{JA}$

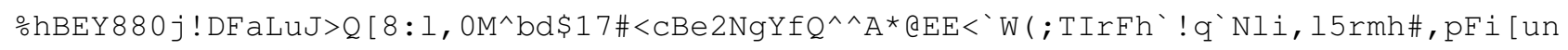
Vb_"TDnQ`p\#Ct2\*N(D

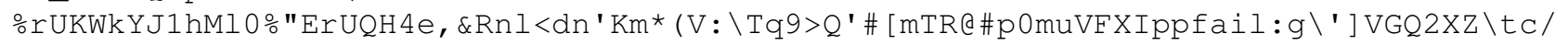
ja4@l 7gGa\%hhnHV33UOT [

\% ' t 3WkkCS, mDJJYebo] O. / ) o_rGt $8: \mathrm{N}-$

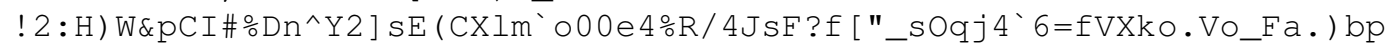

URL: http://mc.manuscriptcentral.com/tandf/tmph 
$\left.\left.\circ \mathrm{C} \& \mathrm{dn}=\mathrm{S}^{\prime} \mathrm{FnT}\right] \mathrm{D}\right)>\& \mathrm{PH}\left(\mathrm{p} 8><\mathrm{Ba} 3 \mathrm{~EB}-4 \mathrm{~g} \backslash \mathrm{RD} \mathrm{D}^{\wedge} \mathrm{YYLK} \square\right.$ 戌」

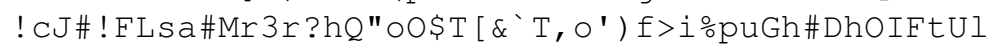

$\circ A C K G[p, C \% h 2 t$ 梿 $>X \backslash p]$ \# ( $>{ }^{*} g \circ T F * I Z O m ;[X M q Y p \$ I] 6 A 2-$

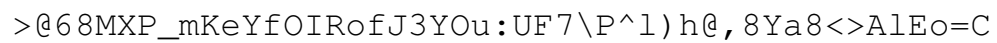

。LRZ?Q*nGK2fDbR; \DFlEg:8,>$NRE] \&2nbSO4\#bp:A`OlCU\#hTi_-

$\# a / ;$ "GUK [K`hk) ‘*1g'V?GPf!nJ\&VKGmTf?BT@u-KXZl, J

or: JZUIbO \ne*ZHcjTBBarTr [Ma", qMAs_D\&Af'bcT09gaZgG?MH`PKi, <gV.k4>_"B91s' 9 ? \, !=:n7

BAmb@_W=g\#) $\& ; \mathrm{mCH} @ \mathrm{p}$

$\because \mathrm{GE} 94 \mathrm{OmbZcIq}=] \mathrm{h} / \mathrm{n}_{-}<\mathrm{k}^{\star \wedge}[2 \mathrm{j} ; \mathrm{p}=[\mathrm{F} " \mathrm{~h} 6-$

U@ro; 2 jmN 48 ieq [ =qZ\%os\#2uEU4rVW8cZ`Ej! dJN;XHg?^jH?5V4Z1cAmIeWMUZ\#6se

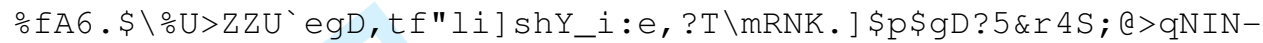

H_75'RWn_n8*C'70ronhZIT.K03(0Aet, >5r@3

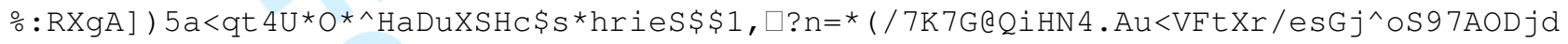
QYLM* $[\mathrm{X} . \mathrm{r} ; \mathrm{C} 1 \mathrm{k} \# "$

ㄴ $40 a V F^{\wedge} \backslash Z \& f>6 "$ ! =LW?S?han`b'E6t`r?\#D\$^NP, "ip)_Wr>TV'RSaMQb/`:g:pg8s, YI]IJ`<Sh7>I IG\$jg4rkI@^fAFf\%]A5^@

$\left.\frac{\circ}{0}=\# \mathrm{YU}\right) \mathrm{kW} / \mathrm{p} \backslash \mathrm{NYE}-\mathrm{gZO} \backslash \mathrm{GO}>\& \wedge$ ] (EJGC\#t?FHI75] 9AiUeR! $1 * q e n-$

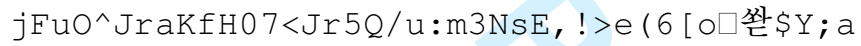

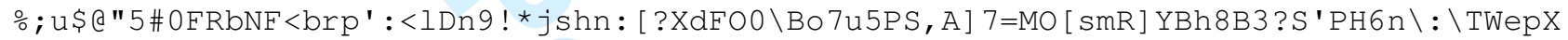
T95] A9 [ZZqJ (1j_eK)

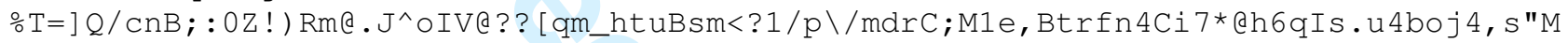
pSrDNQ\#*ru_\&bHdE\#\#

orAfqhr_LT4fG! 0BBm\JCYIH^X6h=h^] t_e, M2u? $\circ$ SR6bc1BCN-

s1CKMa 7g, 53 fm8IBm90Z) LS9Km' IuYme 76 i $=4 \mathrm{~h} * 0-Y a u$

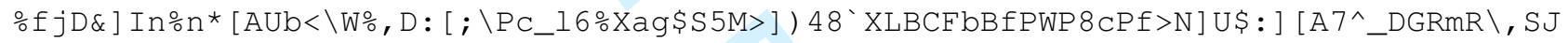
$\mathrm{W}[-\mathrm{V}[\mathrm{ZXV} \cdot \mathrm{kNhs} \mathrm{Q} 2(\mathrm{kZ} ; \mathrm{WH}$

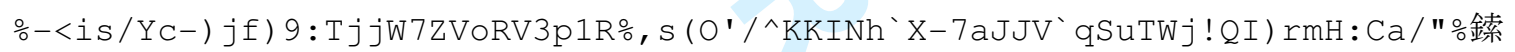

.p34!,mri'>Iou. $1 *$ RJ0jpRcp6

\%ecoii]S] =, 6J@l.mAKp"OsuhgMm\%fluY9hU_>NleVL]BB. IUI] $3^{\prime} \backslash$ GlPtSQgrcoI \$LIVma\&@dlVBA9B ._R\#IaI`nJ $4 \mathrm{~W} / \mathrm{L}[1 \circ \mathrm{A}$

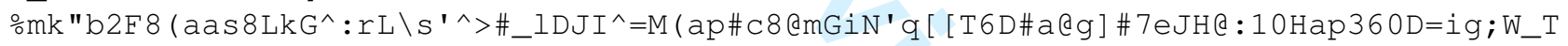
A2pu $8=$ g<OCRqip $6 " T$ !h $\backslash$

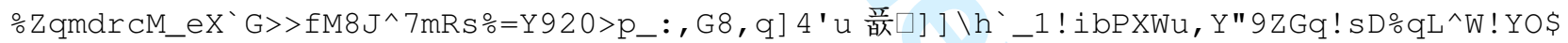
$0<\mathrm{mqDg}{ }^{*} \mathrm{CB} 6 \mathrm{YDfDifK}$

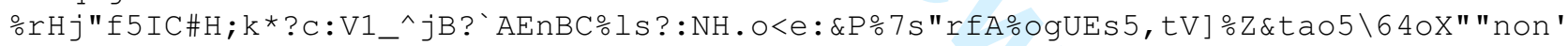
$B * M</$ ue ! p_qnd 7D0D $=<$ '

․ 2<WfMZ ( li (U4p [YLe: \HpLT3kjf/RSM5r:ZJdG4iRnJCjh<mCAYIrO\LfrV:;KqSr\%ZU] 'qJ) Ft=`b $\left.Q " e Q H^{`} @ \# A j P M 5 \& ? N^{*}\right]<$

$\therefore$ pei-

eG \\QpUbqS_:YQ, 56p@N6N`ncai_tMskg! : 8?r.C<LbRrUGS 7n [^\$kK6 lpok]X/Z?pMP6a5Y9; *Vf; C $\mathrm{hnF}>\mathrm{Y} 9$, WZ $>$ Q $>$ SRY

ㅇqoIqX! $[\mathrm{P} \& \mathrm{bGkb} / / \mathrm{b}-$

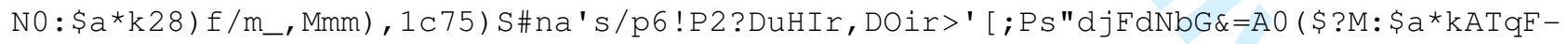

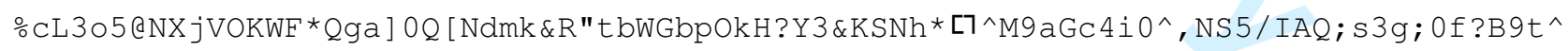
$A Z B * q] B \$ a F 2 @[B Y " @, ~ I$

Igl64"F33die5rVVHspW3 / ?obm) L_WLIHVgDV*bBpFi\#g8 "NcI2\$rR: >rJsK40\#H\b; 6k^h\$/>hHReZ^@o5J-hsqo`ctcuGlIk)

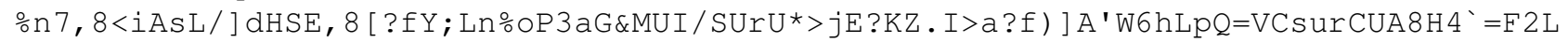
: (\&06 pZ: $\mathrm{nCK} ;(] \circ ; 2 \mathrm{~W}$ \%०9/*U_kLq\$`RHsO/9i8P-

r') Zr(4UR [il.=; TicGTgR! \V\%n1t>SASE0k) 3>] SgoAd\$OVa, B\%\$G) 5MJHLbCai; \$U1G96C_<J 井 ‥"" : [?TIbEn\$XIe3*FD (*2\#HCS, . (_JK1<*nil] I'u_WMC!HOd RaM'b] 71, .oM-

$\mathrm{FR} \$ C C T) Y \circ=\mathrm{LfCm}(6 \$ \mathrm{nuM}: \mathrm{qe}<(0 \mathrm{jNHF} 3 . \mathrm{Xu}$

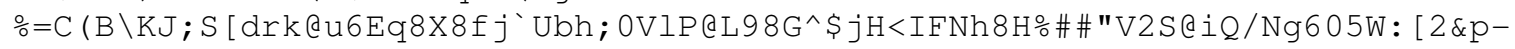
$>$ !’ $=\mathrm{L} \& \# \mathrm{HJ}[\wedge 1 \mathrm{Sq} \cdot \| 47 \mathrm{~T} @ \mathrm{AkC} 1<\mathrm{OU} \backslash 6$ 
kuAoE\$m-/3*"\uCoOD>UY:_C5N*riGfd\$1? 4CC5U/V6T] 8q>5"dKC\#i!L 7r,VAX/I:i/Bl/Zlb"so4ZN ( oK=SUsRY9FP@ 7FY ( HC

ㅇh $=02)(a>n O P 7 \circ) \mathrm{D}=\mathrm{s}>$. ; $\mathrm{p} \& \mathrm{CY} \$ \mathrm{~g}>\mathrm{bF} \backslash \mathrm{H}] 16 \mathrm{~N} \_r e A R b \# b 61 \backslash r-$

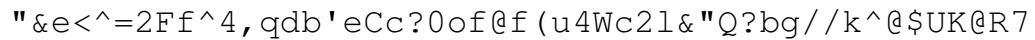

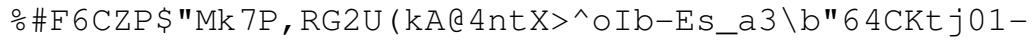

jrI"YA! Ddna`\&6; ] oUS5H\#VK^CZKMYI ' 4Q`3g6b\$ZohDMTLL\&\%Q(

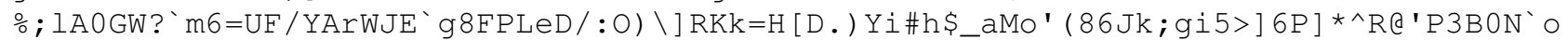
Sf"Y4J0b; * $S^{`}\left(I \backslash ! n !^{\wedge} m t\right.$

。\&IS9YZCeF6L ( jGeGjLZFUXu\$\&f?Js'=7FBSAKN9`Isrs/XZP9k`V*SdIsdRU3neb/UYGm<C;6a\# 4m:3\$'!AQ^.kW1uWNu@2b@o

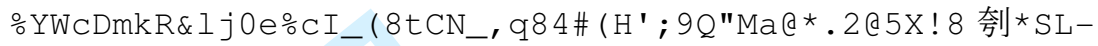

$20 \mathrm{mN}$ ' dR 4 ! pn: UU6eVWUl "\#P; $\varepsilon^{\wedge} \mathrm{T}-5 \mathrm{Ij}={ }^{\wedge} \mathrm{g} \backslash 2 \mathrm{q}$ ? 4 ) \$f\#n) ]

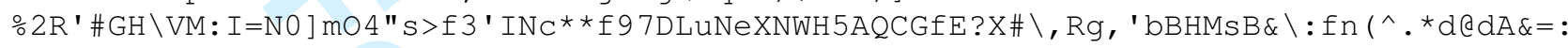
$9 \mathrm{gDOu} \cdot \div$ ? $4 \mathrm{M} \$ \mathrm{IIX}$

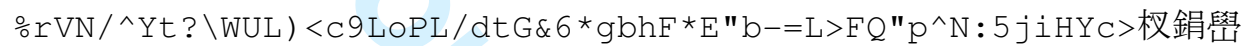

$<^{\prime}<$ ! Kgj\&\$ (\#) Hd"nM<@aX\&tS7.5rCf*C

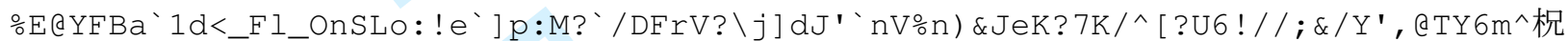
$>\mathrm{sO}<\mathrm{n}, " \mathrm{G} 8 \mathrm{qs} \times 9(\overline{\mathrm{AF} Q}$;

6 WM^G6"tVi 7A\&e?, SFr9MNiEHZ<On/>MZeV4 '<dZU"u*7K*H;Xkl ] t" ' @-

6B] @Bd [\&PCX_s=EO@H : f / . Tt : $V^{\wedge} \mathrm{M} \circ \mathrm{q} 2(5 \mathrm{~g} \$ \mathrm{jHrb}$

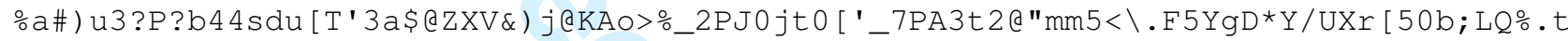
C`g?! ०?M9:2=PtQ=1IRTJ

qM (uYMIsG! "r\$, \$, L\$ [Vg@0jI`hr10>P8_6Z9D-Q2R`Sk11ZPPXBeEXZYGVgeEdcakn>)] KUfE>-

$0 \mathrm{EC} 5 \mathrm{PV} 7>]$ h 4 St $[\mathrm{Qt} \backslash \mathrm{k} \cdot 6 \mathrm{~L} \# 3 \mathrm{C}$

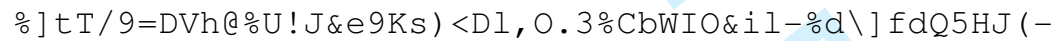

$\mathrm{k}^{\wedge} \mathrm{aN} \wedge \mathrm{ok} \& \backslash \mathrm{S} 1 \mathrm{cN} ! 3 \mathrm{Mb}^{\wedge} \mathrm{a} ? 6$ *.6 T>f'23.2HQk^tLI*mhW [ [ \\#7b

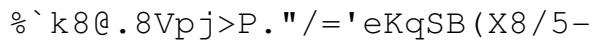

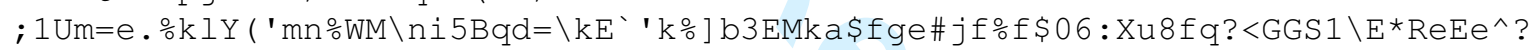

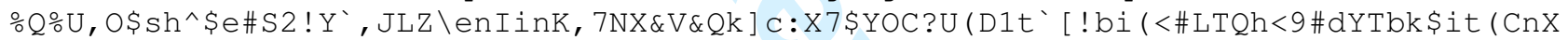
tDdfW*a $4 \backslash \mathrm{QSB} 45 \mathrm{~b}^{`}:$ FO'"

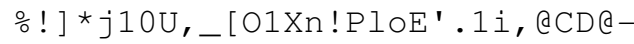

Zez3e\#! [rJ@\#`5q*Ab: *inmuZmZV2b;2G>D! JABVk5 7L [ 1MG\#t\&mD>0E1>9hel 5 c? ?h

애k‥1BhEM\$5a>K/963) FA\$LAmua 4-

nJdh@8>AsZ@n@1 [ * (hk1b<fC'ROt*1GNan4EbQ] UF8f\#="1D;4,2ajTu`C@o`m, 11S*B

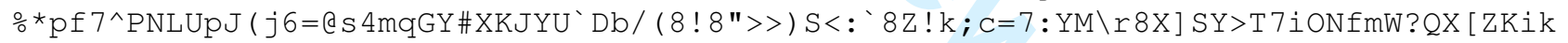
"n-eaFaD! > L//Jg 4

IFtcW:_T.8Md4d?.bO\$fY) P, K [fF' ! m2YN5X1QW) Oc11; jR] sfQX=dYuV=_: *5 'rZ\&D8*e] ZMj9]P7 _VESs, L!DV.G8if7PgVTI

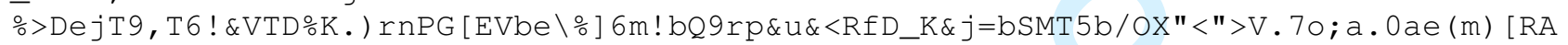
oj?QWP2b\&E7) N]^h! $9 u$

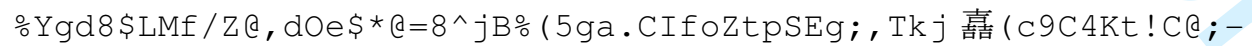

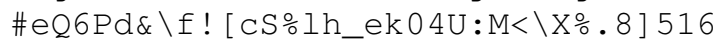

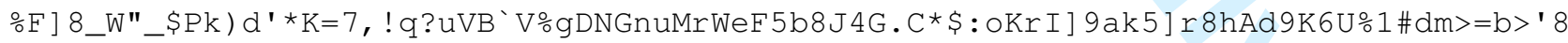

/ Pmn, RO2<<54\#\# . \&\#\$

OZq"ZC"MU: :\$W_g; , T TX?*`qPLNURD ( ) , "so>L-

[Wr (39.SJ4 4<h@\&B4ub`\#; *Uj5a01YLJJ5; b/o! ef 54 ) hoE9NENIL \$d $4: T$

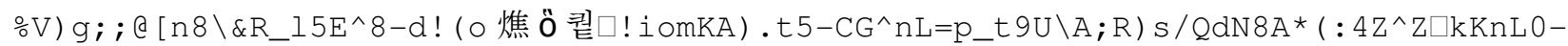

$\mathrm{B} .1 \mathrm{Cq}^{\wedge}<\mathrm{b}^{\wedge} \mathrm{iCA}$

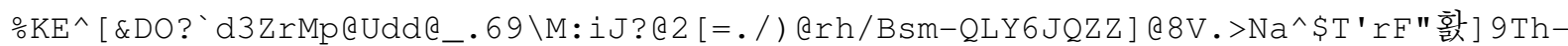

SERZHhC7iV] p<'gJ\$ D $9-$

\% >nA ! J [u:XUBMf *\$oJhgce ( ‘ IK6-VieGPdgI_-B@bA8B3XT8t? 9VHa; \$ ICWrW\% jnH`TRW\$DKMqpG ! -

$\mathrm{F} \% \mathrm{ku} / \mathrm{m}] \circ \mathrm{P}_{-} \mathrm{j}[\mathrm{nsi} \# \mathrm{R}=! \mathrm{r}$,

$\div \mathrm{a} \div \mathrm{A}<\mathrm{DQ}]^{\wedge} \mathrm{X}<\mathrm{N} 5 \mathrm{t}^{\prime}$ 9P\%JqE / , / KqWI\# / P 75=5Vb<F_Q>JJ\&PuZID [OYp*B->Yj-

CONp ) 4sV) di jBUmk 06Nq' ) p\$B 铞> 'cb16 [ :

URL: http://mc.manuscriptcentral.com/tandf/tmph 


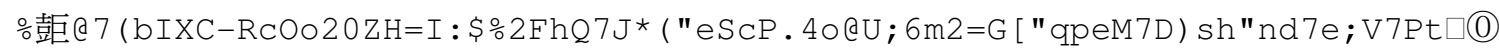
, ! p, /\#IAo_K ( p5\# jC\&! ^* $\div 2<\mathrm{Sf}-$ QJjp6<M(\&ElL5g`FO4>>F(, Gs`j6! 8Z, 3kT? ,VeYWCG2K>bGi6ic\#k>_9<FUIG* \NX9ZAE\%*,Wp]W=2) $\mathrm{fSH} />\mathrm{K} 8 \mathrm{SGC}>\mathrm{SC}-4$

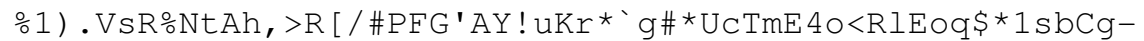
q_NS.C' 'A^Y!Bk'5q]*Pj\#0_92-h*r; DQO<B0pTe'r=C19I

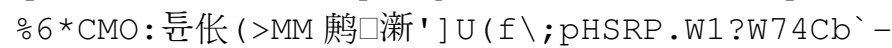
$=5 \mathrm{~s}^{\wedge} \$ \mathrm{~B}^{\prime} \cdot \mathrm{m} 9 \mathrm{RG}>\left[\mathrm{MnAUH} \# 03<\mathrm{uS} \circ \mathrm{Esr} b \mathrm{~KB}[, 9 \# * 8 \mathrm{~B}>2 \mathrm{~d}) 4 \mathrm{jcDVO}>{ }^{\prime}\right.$ 응 1 , KqqG! ; a 7L $>$ " OD0.t\#c! ja< (uX09j!h;\&3; cQ.0KI86A\#KYakQ; ) S) P [C!6c/ ' JRurHYiRA6Kc;S\#S*1P8>\&Sk:t 7=LHn $\mathrm{K}$

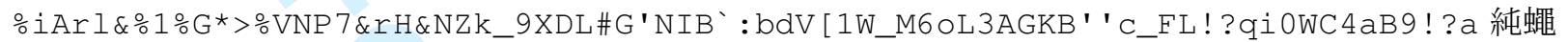
$\square \circ<\operatorname{mb} 9 \mathrm{~T}, \mathrm{ieA} 7><\# / \wedge$

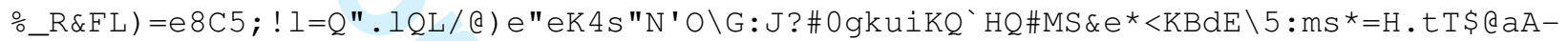
$\circ E^{`} \mathrm{DSY}=\mathrm{ghN} \backslash[5 \mathrm{eRr} . \mathrm{UEP}$ 3] 7'b0_6u:gh) A0 isCCDWuED1d4c [ @At0S//7G4^HCI<FNiGXVUrlELf8 ' Nm1Be$<A] C D \%$ e_WC"\&LI^1/iqnSiNf $\backslash$ f\#.hPmd3\$ \% \WuNGaIkMrPk:(‘ "NnW-\$tbN, gh: [ [n (; D_Bc4; h7 [ ocHUHC [Ru (k) <F Jeor5?gGL8ur) \%C >YkL (eh@, cAKN7O-Dq \9g1kDBPAK E? $\mathrm{aPF}^{\prime}>\mathrm{LX}$ ?a3H7*?1Zm-

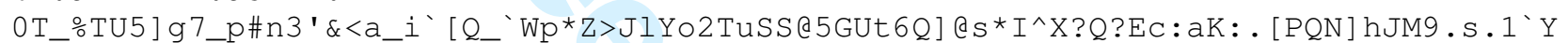
\%_Pdju0i",_9( 7 fEGB\$"n5D; D61GKR﹎V4K!K: *`U6) N9InG3bkjNZ"K3DD; 9>CG4Hf 8\%) Q_MfF5sRD9`uUIt6) 65/nImGdANn ○Y [KGP) U=X/. 8 [ @\&@BtME (Q] ) lq2s-\R6sqUWq4t; 57M9sK<' "ARG' ! h:B*\&gUq1 bpWWs/MU_fX5A1UMM) J $[f \Theta-0:] \backslash \backslash \$ O 3 C O M 8$

ㅇ $(\mathrm{ES}<\& \mathrm{qCK}<* 5 \backslash \$ A 1 \mathrm{Tt}=\mathrm{I}: 6 \mathrm{u}=\mathrm{OKn@mZ}: \mathrm{L} \circ \mathrm{H} * \mathrm{oqp}: \mathrm{Kh} 3 \mathrm{E} 73$ ' G@3) $(-$ t $1 \circ \mathrm{SHK}$ ) eXkPUr>buSVHP^u! ]^[1?) PZ>5<[ ) TA^-[O [^@U [

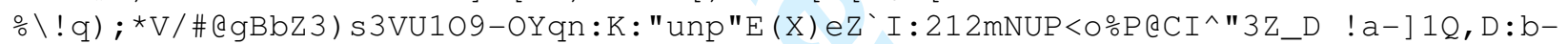
"qe1srD].E\HWghi, J\$;

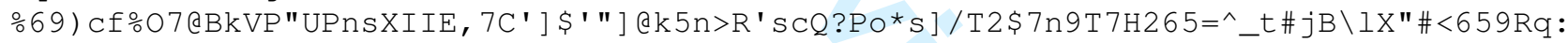
Q (OG' ' @S\# (Yo=i?f

ㅇ, $\left.\mathrm{F}<: \mathrm{B} ! \mathrm{f} " \mathrm{~s}^{\wedge} \mathrm{XBA}=\mathrm{h} !\right)$ fYAJdp $\left.<\mathrm{J} \cong \mathrm{OPn} \$ \mathrm{ps} @ \mathrm{kkKS}\right] \mathrm{EF} . \mathrm{t} \% \mathrm{tAgdm}, \mathrm{E}=\mathrm{Qm} 6 \$ \mathrm{n}-$

$0 \mathrm{f}>\mathrm{E}: \mathrm{H}=\mathrm{OU} @ Y I F f$. ' ( $\mathrm{fYH}<\mathrm{Sn} \backslash 2 \mathrm{~J} @ I$. fDlkm'Z1Rs (B

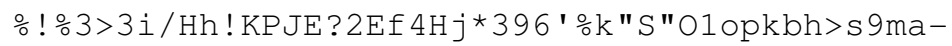
a 0aZ $(\mathrm{g}<\mathrm{j} \& 31$ ! 2h\#t?B 1 [Cd`Db`aMdeCkd'4\#3gR<,r2B:RF; ‘ \&Jq/; 2

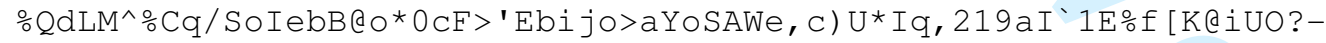
K7O\#UKjIS3CQWeSKeWWVC8q,_i] ! Q> (Z5d7\&०"

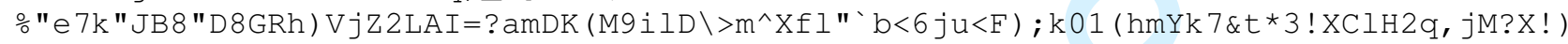
$\wedge \mathrm{O}[(" \mathrm{KC} "=\mathrm{O}] \mathrm{CMk} \# \mathrm{Kt}$ : WR?OT]n2i^ok $9 * /$ dedc -

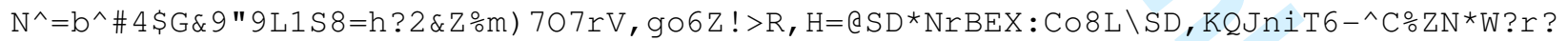
HI $* 3 G *, H 9 F / O I T D J K G h E a s 3 Q C A H T>M 7 f u-2 C ; B q b * C J L$ ! Q -

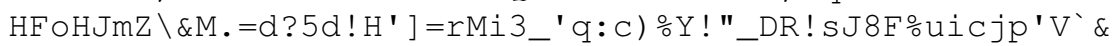
Hm?Mp=S`M/la! '\&pUMG\$:KMXrrA5 (iL:i) 7R*\#b8B?capB<2Gk1Ch?k`k_l;9TL1?!9-

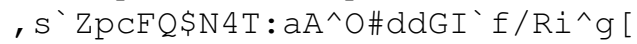
○qf0<.g?ru\&? I* : : * f8rq*q\$or-sSM: , AjjMSA5eoM. KA\#U. UMrV) FJj9] 6=J) PO@/b4 (KU$>\operatorname{XniSK},[(\mathrm{T} J \supset \square \backslash \mathrm{K}), \mathrm{q}$ 으 $)(0: \# \mathrm{E}=\mathrm{nLq}=\mathrm{A}$ * $\mathrm{pe} / \mathrm{fY}$ ?AEIf>L9k6 (MYfG1\%0EAM; q\$G\&M?R*, HO.kFa\$j [U4"<. Oa?k1?q5geV; "] D4 4JmDOeCald! I1] PQ

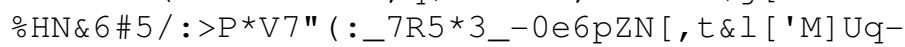
$\mathrm{KC}>\& \mathrm{~nJ} 9 \# \mathrm{~S} ; 7[., \mathrm{r}, \mathrm{t} \mathrm{F}=\mathrm{DQ} * 7=\mathrm{n} \& \mathrm{Kq} 8 @ \mathrm{KrO}=\mathrm{An} @ \mathrm{U} 06 \mathrm{tKaE} 8291 \mathrm{BPV} @ 55$, Ab3

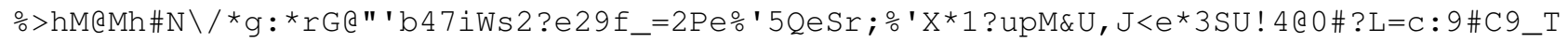
$:{ }^{*} 3 \mathrm{~h} . \mathrm{Opnn} . \mathrm{ZQji}$ 을 $(\mathrm{DR} " \mathrm{~h} 6=\mathrm{Z} 4 \mathrm{X}>4 \&$ ? X8t*XM1BjSU*A@F,F1I.81]2*\&*ih"\#LDA\&] PQP\%F! ZEQiu\pBita'\#) 'ghK/`p (@)dF*'E0) D 
응 ' XKS $5 \backslash$ ak\&-

^ATAWTQFD; $\mathrm{S}_{0}$ ! H<dS=? I=Y=26N\&f79'S ! 2a<9Y*VVUM?\&'m'9] \#FgIq1"q"a2@i ? I63W>:L8f5YSg0\# $\mathrm{Mj}<:$ haV

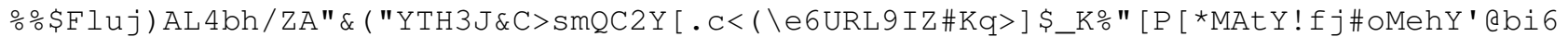
$\mathrm{a} / \mathrm{A} ; 0: \mathrm{qlQ}-\mathrm{O} / \mathrm{RU} 94 \mathrm{G}$;

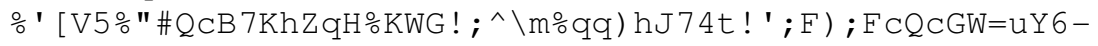

, ; WJg40s`\$ ZZ>bA`fL\&YgjR<\#bA (_??f4tgp^CYhr4joEBQP :

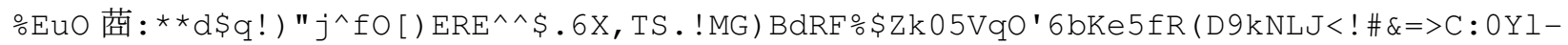

! BQ^r*! $6 \mathrm{QR} \div \mathrm{C}^{\prime}=$ ? [G

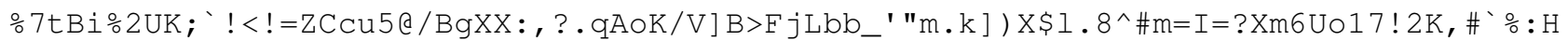
eE/'iaCh [ 7 fV7YFdBPo6 6

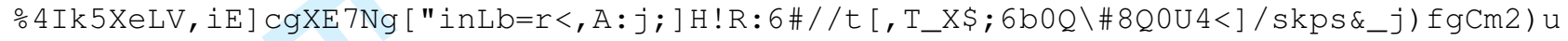
: [19t6MZHG [q?^ btopq1]

\% ! OMG/36sL>k; @ $\mathrm{H}-$ ? IUOFJ3MU -

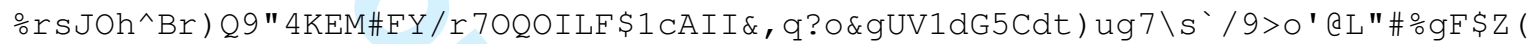
$\circ \circ 3 \mathrm{~S} * \mathrm{~N}-\mathrm{J} 4<\mathrm{kYk} 7-$

J"4PnP! l; lt, ) eo"5T`J4! "4Ii7_l6NOVUL0rUT\%46qH, a\#\&) j*"*\%ffU^] I ] aJ9PYS'rs5-

^ $: \mathrm{rBT}<4[; \mathrm{M} 8 \mathrm{ApB}$

ㅇMMGT.Nbn, $443 j " ; B \$ 8 V^{\wedge} 8() h F C f l F-$

6 (f $5 \mathrm{p} \%$ ' qG8\&e>F) jjRo\#"m^T' $<\mathrm{K}$ ! 5d"3_:m`'cE_oY\#\#F0ZQgZ4\&H\#01F@JP

$\circ \mathrm{BO} / \mathrm{Ca}) ;-$

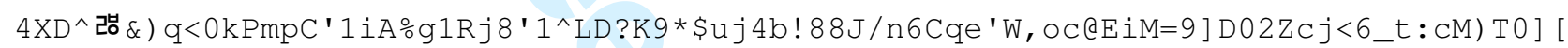
$A j ` f X T=S I F$

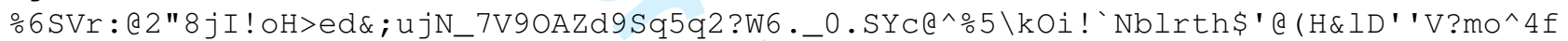
03 CNWIAQaOOqe $=/$ _a $(\mathrm{V}$

^h2/jV.oRf?bc@ZCe; YjO-=Y; g^-5D5d`Zq'825>g! UL ' ?d8b=H:H! ] ‘ KZY-

KdPTUt/ 4 ! qrQJ'POL : QS-bgN<fV/Vb-GOs, H

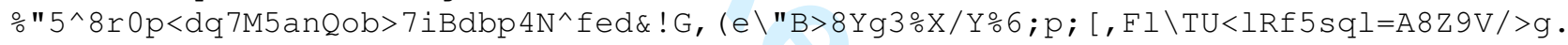
$\left.4 \mathrm{fa} 6 \mathrm{gA}] 4 \mathrm{sp} ; \mathrm{cqO} ; \mathrm{BR} 9 \mathrm{Y}^{\prime}\right]$

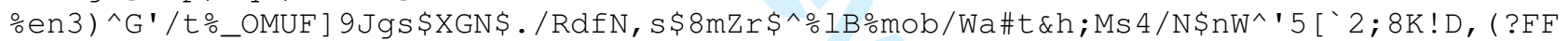
$\left(\square \circ \mathrm{C} ? \mathrm{Bp}^{\wedge} \mathrm{bE}\right)=\mathrm{RH} ; \backslash \mathrm{d}$

의 ]iz01R]n4kgIb>GI [0SM[ \:aQ, 22*771j0Q9'杂

] g [ T [ , E<\#Q Y Y\#NT3 ] mA1FqMO?6G>L05iPTU, 0R>1 ! S`u91FMFWXoV\&XC

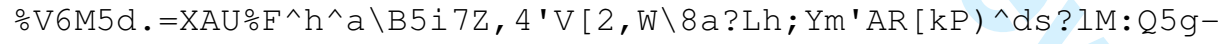

MWs \& SteR@qMg! ; a $5 \backslash \mathrm{PL} 8 \mathrm{~J}^{\wedge} \mathrm{b}>9 \mathrm{iD} / \mathrm{IdI}^{\mathrm{a}} 4 \mathrm{kPI}$

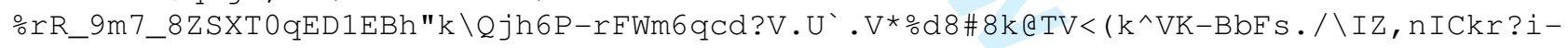
$\mathrm{u}^{\prime \wedge}{ }^{\wedge} 4^{\prime} \mathrm{g} ; \mathrm{k}>\mathrm{hX}>\mathrm{VSWOj}($

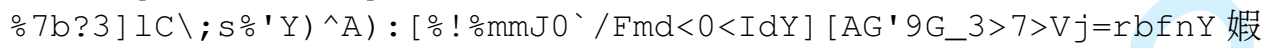

$\left[\mathrm{W}^{\wedge} 4 \mathrm{R}\left(\mathrm{A} 60 \mathrm{~h} 7 \backslash \mathrm{h} ! \mathrm{kE} \mathrm{E}^{\prime} \mathrm{fo} " \mathrm{eB} \cdot 0>/ \mathrm{LG} \mathrm{B}^{\star} ! \mathrm{u} ; \mathrm{Ns}\right.\right.$

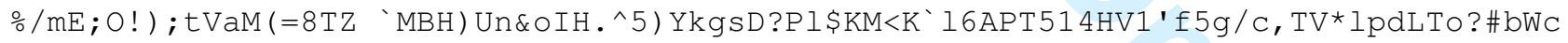
$/ \mathrm{EWdQC}][\& \mathrm{~A}), \mathrm{CMeX}$

\% [ " . <] NgXcSq\#HncmgIQ\%f-PWK \XXe`(ok^OZO0o_>"N6VAjPd` -

. GPf*! t\#bNq\$6KjWMid\%tSb0XXS/ JXC

ㅇ 8 桷 $\left(\mathrm{YU}^{*}\left[7_{-}: \mathrm{YHF}, \mathrm{T} 7 \mathrm{M} \_\mathrm{b} 7 \mathrm{MbBP}=\mathrm{Y} 1 " 11<4 \$ \mathrm{gO}-\right.\right.$

oPq6g5 ( ] gs 9) q_jHQGe, aBm, ?PoutWP<kS60\$1\&O) G ! J , I [EtM, 2pbeO (>MjN

유 : g"etRM'FsW! "S07InT! j3q-

7n [MiR5\$DYDJjB. @, JeAm'd_* (b?o*8: JiRQ\%GmJrkuQkP1nqb\%Zu\&/T5\#u:C6ufM3c3uDUi6H

7 $\mathrm{ckT}$ *"] qQ) $\mathrm{s},>\mathrm{T}\left(5 \mathrm{~W} \_\mathrm{aGFD}\right]<$ Vg_4ESjEr:Y, '\$6NZcMe\#daF-

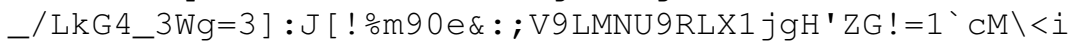

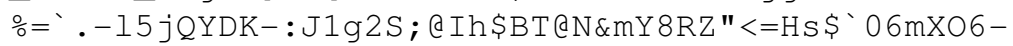

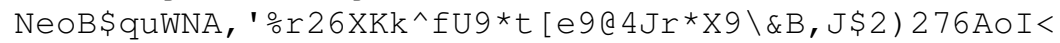

V $\$ 1 T X \_b ! Z s F(e)=' ;-, X f \$ \backslash R K /{ }^{\prime} J 0 c>$ 'ALtZ; d: [fp; ! a/5I^UnZG\#\&' ! SsUJX1LfO [sa-D=\#, (5apYA; t, s09sZte-35N>dBXM

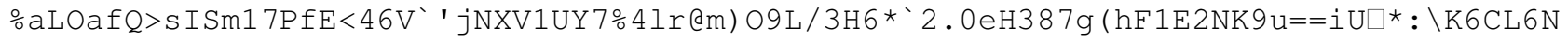
J1-S2AL : qk "D@ "-

URL: http://mc.manuscriptcentral.com/tandf/tmph 
으); ; 2He) ?bd \WeZpAoS) B 7fBpdD115e [\$ki8eD\$0?/ (G, e ' ?MMI, " E>>3NdhpuXh^_p>YBQ6;'n.YB4115*s (>HppP (*=: / ! @

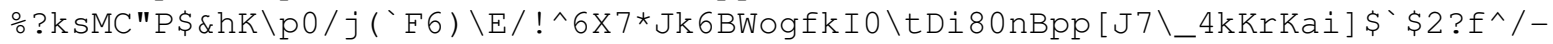
$=\& q(:$ ZBUhKm $>\mathrm{G} ; \mathrm{r} 6, \backslash \mathrm{hl} 5[\mathrm{R}) \mathrm{NLQ}$

○OaRj) , O GHrna2\$fNE><emK9 fJ35E\%/) YQNmd [B) 3fsLBN<bM5\%j6XU* ( "X52\#Qpdog\#Q, *R [ oE/>K1 $<\mathrm{XRC} * \mathrm{EOg}(\mathrm{X} !,<.8 ; \$ \mathrm{k}$

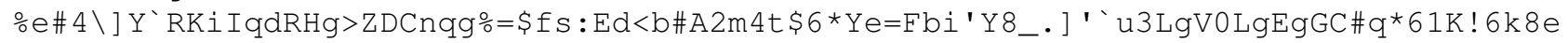
$\mathrm{ZH} \mathrm{H}^{\prime}$, , \&m $3 \mathrm{n} 7 \mathrm{~b} ; \mathrm{SOL}$

응 is; 2 ZZpluH0p-ec=u3

\%gp" bmH 2 t $4 \mathrm{~J}-\mathrm{kBif}-\mathrm{srQ} 8 \mathrm{PG} 5-$

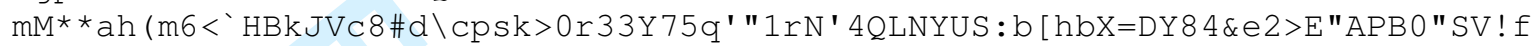

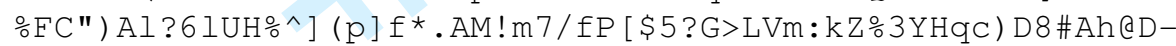

$\mathrm{F}$ ?X(XL.?CHth90Q: $\mathrm{Q00} / \mathrm{hL} " \mathrm{~W} 6 \mathrm{Cbr} / \mathrm{kk} \backslash[[2 @ \mathrm{f}:$

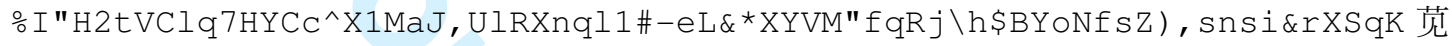
_P:Zn_fT\3)_55TEB\&Gu/e3J_1hd

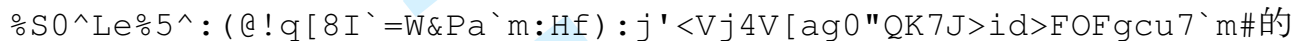

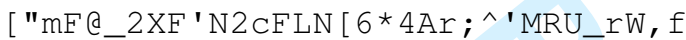

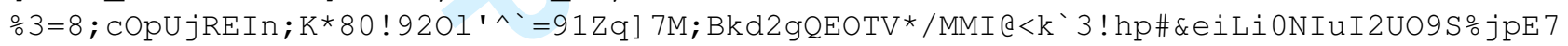
$\mathrm{hWa} / \mathrm{dHr}<\mathrm{D} 1 \mathrm{~B} \backslash-=^{\prime}$ HbAtbg

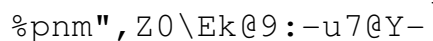

ZAa6`7 [P3Iq\%I] 298 ! OHqP*IHPpoL; @BgaQ (3P_VIQWqsK=tZJ) I] SmI2aE9kYu\&G: [eK. RS 7P8t ?Jb; 1

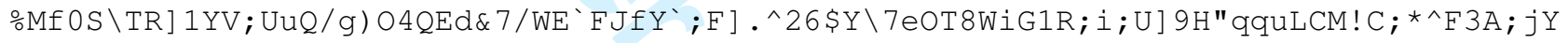
hs! Jb2z/S45Z97[/g

。Bb\#.H\&KIVTK $(<)$ i_QS5AOsO->?D"^\#Vr-@.BM'-KGcO@seQG :呾

뮬) $\mathrm{L} \backslash \mathrm{U}_{-}, \mathrm{Q}=\mathrm{Q} \backslash,>\mathrm{Q}$ ?Mt \& / 76 "_YfFYD $4 \mathrm{QVk}:$ BW_T`SpJu 9

○>ba] "qT7*L8'-<86c'?1M4@ApDnTi \/EI . [QHePA5*-D@UXRk*iV-Wd"Or.\#m018c .; $5 \mathrm{I} \backslash \mathrm{EEaen} 7$ [P_PWd $4 \backslash \_V h, \backslash \mathrm{k} \% \mathrm{M}, \mathrm{CI}$

F"A? 55\$gLO2Q9 [C= [UrWaJBg. U. 1>9 [P<?edI: (b\#pW\&* ! ZtE () "d0 ?bu6q\$08Fps. N5 "; u ' 36] 6qnN $\mathrm{bJ}>\mathrm{g} 1[\mathrm{nQC \#} \& \mathrm{c}$ ! PX\% . ER2

․ OD@ [dNfa/8nab [r`VOAG5 (0^aL^3*Dgkdii\#/F=(7.\#glgTUVLtg9D (LV.91800 [Y?u [-

iRiscUm $4 *<3<\&$ TS $5<\mathrm{U}^{\prime}$ [ ? ? ' $\$ C$ CNN__)

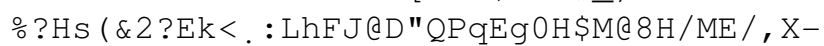

$\left.: \mathrm{k}>\ldots .>\operatorname{pDr} 2 \mathrm{kUpo}: \mathrm{S} 1 \mathrm{a}{ }^{\star} \mathrm{Eb} \$ \mathrm{~g} 8 \mathrm{WnKN} \mathbf{M}^{\prime} \mathrm{M}\right] \mathrm{ZG} 6 \mathrm{gI}$ ?mEIEO6 [2D'eDO?npNS

$\circ$ DqL $[$. HM $\%$ S $\$-X,-$

^/_OmZgEd[40@2]VRCjteRIZPX0\C03r3'dG7] OX3005\&Q7, 5, 7?nb, ac, D" 46fU3Xplh^[Ylhu, 'h7E $\mathrm{X}($

] A90b] Ckr@ \, NIAI>m*LI^e?dbGMe \NhCB_: \$bA>\&@3; 8 . ukafojb, "fo-

$=:$ _71G@RZW $\backslash$ OHnra50/bU6`h) lmC/6\&\u_fpA.V

○JaLCThtU) $20: ">` \& \mathrm{~V}, \mathrm{OL}-$

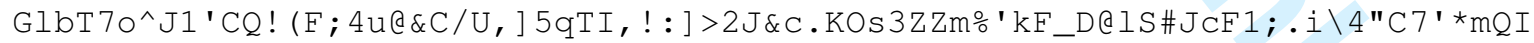

hB ! a; @ Z/j.PAj3 (QMj5gmuNDiGpX<fKII\$LNrA^!) (d`mfYouQYpP5@5 ( [K11QB6TR97AT jGR^A0\&XRj $\left.\mathrm{jB} \cdot{ }^{\wedge} ` \& R 0 * ! \mathrm{NOY}\right] \mathrm{R} 80 \backslash$, ' -

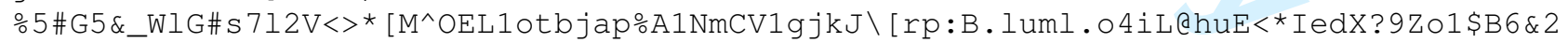

"r3X

$\circ \mathrm{C}=\mathrm{SS}: \mathrm{Q} 4 \mathrm{Wi} 0\left[6 \mathrm{~N} \backslash \mathrm{g} *\left(4{ }^{\prime}-\right.\right.$

@C9@ [\%nqWFF7/s7WCj<91'OCQ; i j4N:OK? \_C (uK_>1Q9Nj!W<5AN) 'SLR/nnleR>pkoU\%YC9NkKeK\%J

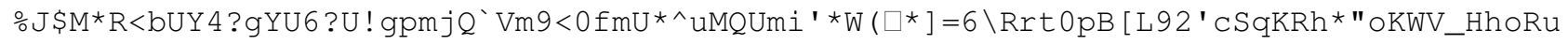
$\& \mathrm{Fj} 8 / 91 \mathrm{p} 9$ eeCqf $[\mathrm{DC}$

XOGXIB@F? ImAU>fB ( ] ZYrgHG : T>/h7eluNTjl<s3pK\#>uh ; 96*_\&_WIr.f4^MdLl*jkme^gVO^4<$\mathrm{S} \sim>$

AI9_PrivateDataEnd

URL: http://mc.manuscriptcentral.com/tandf/tmph 